\title{
SERIES
}

Alpine Forschungsstelle Obergurgl

Band 2

innsbruck university press

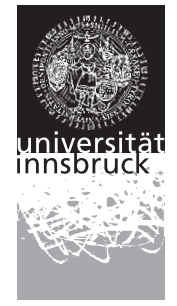


Dankesworte

Die Herausgeberinnen bedanken sich sehr herzlich bei allen Autorlnnen für die gute Zusammenarbeit und die Bereitschaft dieses Buch mitzutragen. Ein besonderer Dank ergeht an den Vizerektor für Forschung der Universität Innsbruck, Herrn Univ.-Prof. Dr. Dr.h.c.mult. Tilmann Märk, für die Finanzierung des Buches. Der Druck des Buches wurde außerdem durch die Forschungsförderung der Hypo Tirol Bank unterstützt. 
Eva-Maria Koch, Brigitta Erschbamer (Hg.)

\section{An den Grenzen des Waldes und der menschlichen Siedlung}




\section{Eva-Maria Koch}

Alpine Forschungsstelle Obergurgl, Universität Innsbruck

\section{Brigitta Erschbamer}

Alpine Forschungsstelle Obergurgl, Universität Innsbruck Institut für Botanik, Universität Innsbruck

Diese Publikation wurde mit finanzieller Unterstützung aus den Fördermitteln des Vizerektorats für Forschung der Leopold-Franzens-Universität Innsbruck gedruckt.

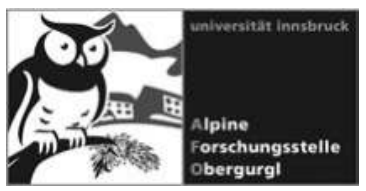

(C) innsbruck university press, 2012

Universität Innsbruck

Alle Rechte vorbehalten.

Das Werk ist urheberrechtlich geschützt. Die Rechte für Nachdruck, Übersetzung sowie die Entnahme von Abbildungen und Bildern liegen bei den Herausgeberinnen bzw. bei den jeweiligen AutorInnen. Die in den Beiträgen geäußerten Meinungen müssen nicht mit denen der Herausgeberinnen übereinstimmen.

Umschlag: Ivan Leuzzi

Umschlagmotiv: Bergmahd "Stoankofl“ oberhalb vom Beilstein - im Hintergrund der Gurgler Ferner (Tal), darüber Schalfkogel, Kleinleiten-Spitze und Querkogel

Foto: Brigitta Erschbamer (22.08.2011)

Layout: Carmen Drolshagen

Produktion: Sterndruck GmbH, Fügen

www.uibk.ac.at/iup

www.uibk.ac.at/afo

ISBN 978-3-902811-40-0 


\section{Inhaltsverzeichnis}

Einleitung

Eva-Maria Koch, Brigitta Erschbamer

Kapitel 1 I Subalpine Wiesen und Weiden - die Kulturlandschaften

der subalpinen Stufe

Roland Mayer, Fabian Nagl, Brigitta Erschbamer

Kapitel 2 I Die Landschaft und ihre Namen - Landwirtschaftliche

Nutzung und Nutzungsänderungen im Spiegel der Flurnamen in

Obergurgl und Vent (Ötztal)

Lorelies Ortner, Rüdiger Kaufmann, Yvonne Kathrein, Johanna Pidner

Kapitel 3 | Zum archäologischen Fundbild in Obergurgl

Alexander Zanesco

Kapitel 4 I Lärchen-Zirbenwälder und Zwergstrauchheiden 99

Roland Mayer, Brigitta Erschbamer

Kapitel 5 | Wald- und Waldgrenzforschung in Obergurgl - Vergangen-

heit und Zukunft

Robert Jandl, Andreas Schindlbacher, Silvio Schüler, Dieter Stöhr

Kapitel 6 I Jahrringuntersuchungen an rezentem und subfossilem

Holzmaterial aus dem Raum von Obergurgl - Klimaanalysen und

holozäne Waldentwicklung

Kurt Nicolussi, Andrea Thurner 
Inhaltsvzerzeichnis

Kapitel 7 I Zur Diversität der Flechten und Moose der subalpinen Stufe im Raum Obergurgl

Georg Gärtner, Wolfgang Hofbauer

Kapitel 8 I Mineralogie und Petrologie im Übergangsbereich

zwischen Ötztal Kristallin und Schneeberger Zug im hintersten

Ötztal (Timmelsjoch, Österreich, Italien)

Peter Tropper, Kurt Krenn, Georg Hoinkes

Anhang 211 


\section{Einleitung}

Die Lebensräume an den Grenzen des Waldes und der menschlichen Siedlung bieten spannende Forschungsfragen für verschiedenste Fachrichtungen. Im inneren Ötztal werden diese Lebensräume in der subalpinen Höhenstufe seit mehr als 50 Jahren erforscht. Dieses Buch ist all jenen gewidmet, die Obergurgl (Abb. 1) und Vent als ,ihre“ Untersuchungsgebiete ausgewählt haben. Einige von ihnen stellen in diesem Buch die Ergebnisse ihrer wissenschaftlichen Tätigkeit vor. Alle Artikel wurden fachlich begutachtet. Wir bedanken uns an dieser Stelle ganz herzlich bei den Gutachterinnen und Gutachtern für ihren Einsatz.

Kapitel 1 beginnt mit den Kulturlandschaften, den subalpinen Wiesen und Weiden ( $\rightarrow$ S. 11). Detailliert werden diese vom Menschen wesentlich geprägten Lebensräume beschrieben und der Zusammenhang zwischen menschlicher Nutzung und dem pflanzlichen Arteninventar aufgezeigt. In Kapitel 2 dient die Namenforschung als Spiegel für die landwirtschaftliche Nutzung und auch der Nutzungsänderungen im inneren Ötztal und klärt mit Hilfe zahlreicher Flurnamen auf, inwiefern Sprache und Ökologie einer Region zusammenhängen ( $\rightarrow$ S. 39). Die archäologischen Forschungsarbeiten, die in Kapitel 3 präsentiert werden, ge- ben einen Einblick in die Jahrtausende lange Geschichte, die der Mensch in den Zentralalpen vorzuweisen hat. Zahlreiche Funde, z.B. vom Beilstein, helfen den Archäologen und Archäologinnen ein Bild der Vergangenheit zu zeichnen: Dieses Bild vermittelt, wie unsere Vorfahren in den Zentralalpen gelebt und auch damals schon ihre Umwelt geprägt haben $(\rightarrow S$. 73).

In Kapitel 4 werden zwei weitere Lebensräume, die Zwergstrauchheiden und die Lärchen-Zirbenwälder, dargestellt $(\rightarrow$ S. 97). Letztere dienen seit den 1960er Jahren als Untersuchungsgebiete für intensive wissenschaftliche Arbeit. Kapitel 5 beschreibt die umfangreiche historische und aktuelle Forschungsarbeit im Waldgrenzgebiet in Poschach bei Obergurgl $(\rightarrow$ S. 123). Dass Waldgrenzbäume Wissenschaftlerinnen und Wissenschaftlern auch helfen können, die Klimageschichte der Region nachzuvollziehen, wird in Kapitel 6 anhand der Jahrringuntersuchungen präsentiert $(\rightarrow$ S. 145).

Auf oft unscheinbare, ganz spezielle Gruppen von Lebewesen im subalpinen Bereich wird in Kapitel 7 eingegangen. In diesem Beitrag wird die Artenvielfalt der Flechten und Moose aufgezeigt $(\rightarrow$ S. 161). Abschließend fasst Kapitel 8 alle 


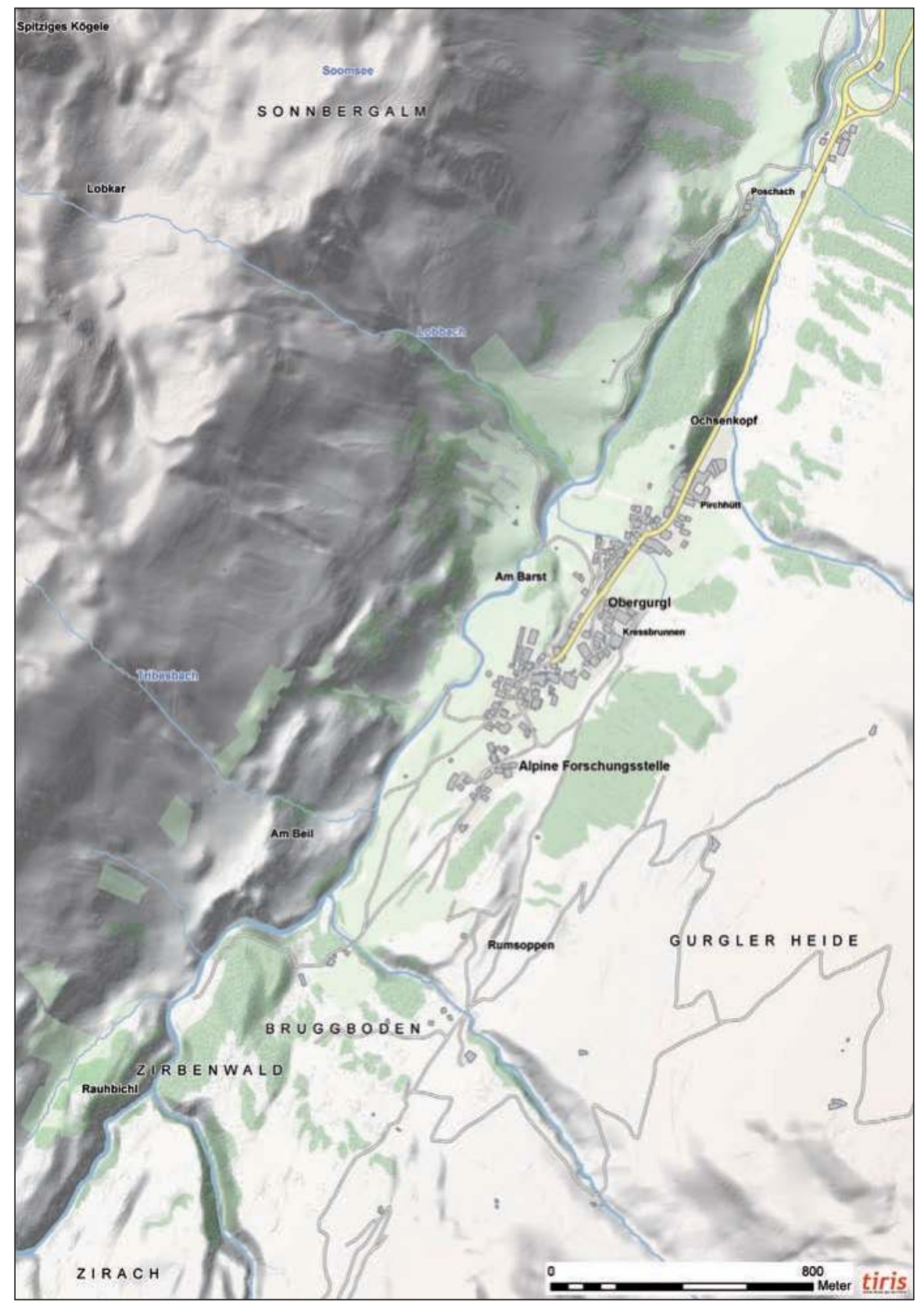

Abb. 1:

Karte von Obergurgl und Umgebung - In dieser Gegend wurden zahlreiche der im Buch vorgestellten Forschungsarbeiten durchgeführt. Quelle: Grundkarte Land Tirol - tiris. 
mineralogischen und geologischen Aspekte des inneren Ötztales zusammen $(\rightarrow$ S. 179).

Wie bereits mit unserem ersten Werk „Glaziale und periglaziale Lebensräume im Raum Obergurgl“ (2010) möchten wir auch mit dem vorliegenden zweiten Band unserer Serie breitgefächerte und verständlich aufbereitete, wissenschaftliche Informationen über die Lebensräume im inneren Ötztal bieten und alle unsere Leserinnen und Leser dazu inspirieren, noch vorhandene Wissenslücken zu schließen. Weitere Details zu unserer Arbeit und den Veranstaltungen an der Alpinen Forschungsstelle Obergurgl finden Sie auf unserer Homepage (www.uibk.ac.at/afo).

Eva-Maria Koch \& Brigitta Erschbamer Alpine Forschungsstelle Obergurgl Universität Innsbruck 


\title{
Kapitel 1 I Subalpine Wiesen und Weiden - \\ die Kulturlandschaften der subalpinen Stufe
}

\author{
Roland Mayer, Fabian Nagl, Brigitta Erschbamer
}

\section{Zusammenfassung}

Die subalpinen Wiesen und Weiden in Oberund Untergurgl wurden mittels 91 pflanzensoziologischer Aufnahmen untersucht. Sie gliedern sich in ein Sieversio-Nardetum strictae (Bürstlingsrasen) und in ein Trisetetum flavescentis (Goldhaferwiesen). Innerhalb dieser Assoziationen wurden verschiedene Subassoziationen unterschieden. Die Bürstlingsrasen werden mit Pferden und Rindern beweidet oder sind Brachen verschiedenen Alters. Die Goldhaferwiesen werden hingegen bis zu zwei Mal im Jahr gemäht und gedüngt. Durch die unterschiedliche Bewirtschaftungsintensität ist das Sieversio-Nardetum strictae signifikant artenreicher als das Trisetetum flavescentis. Innerhalb des Trisetetum flavescentis fällt ein Höhengradient des Artenreichtums auf: mit zunehmender Meereshöhe nimmt die Artenzahl zu. Die typische Subassoziation im Talgrund ist außergewöhnlich artenarm. Der Einfluss der Bewirtschaftung wird durch die Standortfaktoren Meereshöhe, Hangneigung und Exposition überlagert. Die aufgelassenen Flächen verbrachen mit Zwergsträuchern, die Artenvielfalt bleibt aber sehr lange auf einem hohen Niveau. Eine Änderung der Arten- zusammensetzung erfolgt nur sehr langsam im Verlauf von mehreren Jahrzehnten.

\section{Abstract}

The pastures and meadows in Ober- and Untergurgl were investigated by 91 relevées. A Sieversio-Nardertum strictae and a Trisetetum flavescentis were distinguished. These associations were differentiated into several subassociations. The Sieversio-Nardetum strictae is grazed by cattle and horses, however, many of these grasslands were abandoned. The Trisetetum flavescentis is fertilized and mown once or twice a year. Due to the different management intensity, the biodiversity of the Sieversio-Nardetum strictae is significantly higher compared to the Trisetetum flavescentis. With increasing altitude, species number increases in the Trisetetum flavescentis, the typical subassociation on the valley bottom being exceptionally species-poor. The impact of land-use was interfered by the environmental factors altitude, inclination and exposition. Abandoned grasslands are invaded by dwarf shrubs; however, biodiversity remains relatively constant.Chan- 
ges in species composition occur very slowly after several decades.

\section{Einleitung}

Die Kulturlandschaft im Waldgrenzbereich der Alpen wird von Bergwiesen und -weiden geprägt (Tasser et al. 2009). Sie sind in der Regel überaus reichhaltig strukturiert und zählen zu den artenreichsten vom Menschen geschaffenen Ökosystemen (Maurer et al. 2006). Die Berglandwirtschaft weist eine sehr lange Tradition auf und reicht bis in einen Zeitraum vor mehr als 5000 Jahren zurück (Patzelt 1996). Um Kulturflächen in der subalpinen Stufe zu gewinnen, wurden bereits in der Bronzezeit und verstärkt $\mathrm{ab}$ dem Mittelalter subalpine Wälder (vor allem Lärchen-Zirbenwälder) gerodet und die Waldgrenze wesentlich herabgesenkt (Reisigl \& Keller 1999). Der ökologische Wert von traditionell bewirtschafteten, extensiven Graslandgesellschaften in den Hochlagen ist kaum zu überschätzen, da sie eine hohe Biodiversität (z.B. Grabner \& Heiselmayer 2002, Mayer \& Grabner 2004, Rudmann-Maurer et al. 2008) und seltene Pflanzengesellschaften aufweisen (Lüth et al. 2011a,b). Zudem wirkt sich die traditionelle Bewirtschaftung günstig auf die Stabilität des Bodens aus und erosive Prozesse werden deutlich eingedämmt (Tasser et al. 2003).
Seit etwa Mitte des 20. Jahrhunderts vollzieht sich jedoch eine drastische Änderung der Wirtschaftsstruktur in den Berggebieten (Bätzing 1996). Die wirtschaftlichen Rahmenbedingungen haben sich grundlegend verändert und die traditionelle Weise der Bewirtschaftung wurde immer weniger rentabel (Dierschke \& Briemle 2002). Das führte zur Intensivierung der Landwirtschaft in Gunstlagen, während steilere Flächen, die von den Dauersiedlungen entfernt liegen, aufgelassen wurden. Die zunehmende Verbrachung ehemals traditionell bewirtschafteter, artenreicher Bergwiesen und -weiden hält an (Tasser et al. 2002, Tasser et al. 2007). Die Auflassung oder Intensivierung der Landnutzung bewirkten eine deutliche Veränderung hinsichtlich Artenzusammensetzung, Artendiversität und Struktur der Vegetation in der subalpinen Stufe (z.B. Tasser \& Tappeiner 2002, Niedrist et al. 2009).

In Obergurgl wurde Weideland nachweislich schon in der zweiten Hälfte des 4. Jahrtausends vor Christus geschaffen und genutzt. Eine dauerhafte Besiedlung des inneren Ötztals ist jedoch erst seit dem Mittelalter historisch belegt (Patzelt 1996). Die Nutzung des Kulturlandes als Bergmahd erfolgte ab dem 16. Jahrhundert (Patzelt 1996). Während bis ins 20. Jahrhundert hinein die traditionelle Bewirtschaftung vorherrschend war, ergaben sich nach dem Zweiten Weltkrieg grundlegende Veränderungen. Seither erlebt der Wintertourismus einen kontinuierli- 
chen Aufschwung (Ebus 1986, Grabherr 1987). Die Landnutzung erfolgt nun vor allem nur noch in Gunstlagen, während schwierig zu bewirtschaftende Flächen nach und nach aufgelassen wurden (Nagl 2009).

Ellmauer (1995) untersuchte in den 1990er-Jahren punktuell Wiesen und Weiden in Ober- und Untergurgl. Eine umfangreichere pflanzensoziologische und standortökologische Erfassung wurde erst von Nagl (2009) durchgeführt. Mayer et al. (2009) untersuchten im Rahmen eines Langzeit-Monitorings die Auswirkungen der Auflassung der Beweidung von der subalpinen in die obere alpine Stufe. Solche Studien sind im Besonderen in Naturschutzgebieten wie etwa dem Natura 2000-Gebiet Ötztaler Alpen wichtig. Bergmähder zählen zu den schützenswerten Habitaten, welche durch das europäische Schutzgebietsnetzwerk Natura 2000 gesichert und erhalten werden sollen. Die Voraussetzung dafür ist jedoch, dass die traditionelle Art der Bewirtschaftung auch in Zukunft fortgeführt wird.

Die vorliegende Studie soll den Zusammenhang zwischen Nutzungsweise und Artenzusammensetzung der Bergwiesen und -weiden in Obergurgl aufzeigen. Dazu wurden unterschiedlich genutzte Grünlandgesellschaften analysiert und die Standortfaktoren in Beziehung zur Ausbildung der Vegetationstypen gesetzt. Das Ziel war, die charakteristischen Unterschiede zwischen den Bewirtschaftungsformen herauszuarbeiten und Vergleiche mit aufgelassenen Flächen durchzuführen.

\section{Untersuchungsgebiet}

Die Studie umfasste den Bereich von Obergurgl und Untergurgl (inneres Ötztal) von 1800 bis $2190 \mathrm{~m}$ Meereshöhe auf der orographisch linken Talseite des Gurgler Tales. Das Gebiet beginnt in Untergurgl (1800 m Meereshöhe) und reicht über den Weiler Poschach (1830 $\mathrm{m}$ Meereshöhe) bis hinauf zu den Weixelmähdern auf 2190 m Meereshöhe (Abb. 1). Das Relief des Untersuchungsgebietes wird von sanfteren Geländeformen geprägt, da das Hauptausgangsgestein (Biotit-Plagioklasgneis) sehr leicht verwittert (Aulitzky et al. 1961). Das Gelände ist nicht in Seitentäler gegliedert und es herrschen Steilhänge vor. Von Poschach bis etwa auf die Höhe des Ochsenkopfes ist der Hang bis etwa 2500 m gleichmäßig steil. Von dort bis zum Zirbenwald steigt das Gelände bis auf $2100 \mathrm{~m}$ hingegen treppenartig an. Im unteren Hangbereich kommt Bergsturz- und Schuttmaterial vor. Danach folgt auf einen steilen mit Felsen durchsetzten Anstieg der Talflanke auf ca. $2000 \mathrm{~m}$ eine Verflachung mit zum Teil rückläufiger Hangneigung. Der sich danach anschließende Abschnitt bis zu den Weixelmähdern ist wiederum durch treppenartige Strukturen mit ter- 


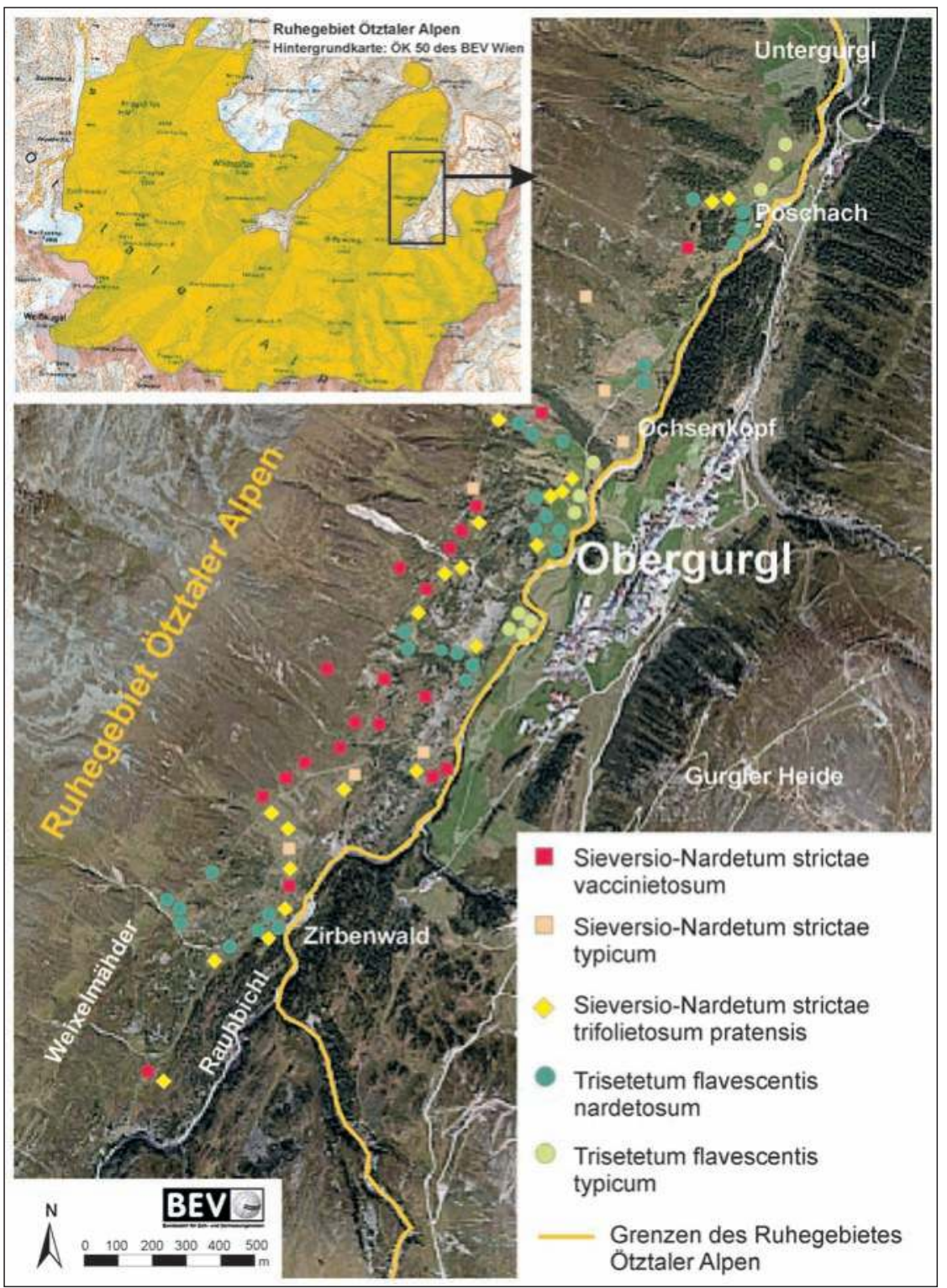

Abb. 1:

Die Position der Aufnahmen im Untersuchungsgebiet. Die verschiedenen Symbole zeigen die Zuordnung der Aufnahmen nach der TWINSPAN-Analyse. Die Aufnahmen von Ellmauer (1995) wurden nicht verortet. Die Lage des Untersuchungsgebiets im Ruhegebiet Ötztaler Alpen ist aus der eingeblendeten Karte zu ersehen. (C) BEV 2011, Vervielfältigt mit Genehmigung des BEV - Bundesamtes für Eich- und Vermessungswesen in Wien, T2011/80692) 
rassenförmigen Bändern charakterisiert. Oberhalb des Untersuchungsgebietes in ca. $2500 \mathrm{~m}$ Meereshöhe ist eine deutliche Geländekante ausgebildet, an die eine Verflachung - die so genannte Seenplatte (Am Soom) - anschließt (Nagl 2009). Das Untersuchungsgebiet befindet sich zur Gänze im Ruhegebiet Ötztaler Alpen, das 1981 eingerichtet wurde. 1995 wurde es als Natura 2000-Gebiet ausgewiesen (Abb. 1).

\section{Methoden}

\subsection{Erhebungen im Gelände und Daten- bearbeitung}

Die landwirtschaftlich verschieden genutzten Wiesen und Weiden in Obergurgl wurden im Jahr 2007 pflanzensoziologisch untersucht (Nagl 2009). Einzelne Aufnahmen stammen aus dem Jahr 1991 (Ellmauer 1995). Insgesamt wurden 91 Aufnahmen für die Analysen verwendet, welche dem Minimumareal (16-25 m²) entsprechen. In den Aufnahmeflächen wurden alle Arten von Gefäßpflanzen erfasst und ihre Artmächtigkeit mit Hilfe der kombinierten Abundanz-DominanzSkala von Braun-Blanquet (1964) erweitert nach Reichelt \& Wilmanns (1973) geschätzt. Die Nomenklatur der Arten richtet sich nach Fischer et al. (2008).
Es wurden verschiedene Umweltparameter wie Meereshöhe, Neigung $\left(^{\circ}\right)$ und Exposition erhoben. Die Exposition wurde in einen so genannten "Hitze-Index“ umgewandelt (Austrheim et al. 1999). Eine Exposition von 225 GON (SSW) wurde als für das Pflanzenwachstum am günstigsten betrachtet und erhielt den Hitze-Index 0. Demgegenüber galt eine Exposition von 25 GON (NNO) als am wenigsten günstig und erhielt den HitzeIndex 200. Alle dazwischen liegenden Werte wurden linear aus den Expositionen abgeleitet (Austrheim et al. 1999).

Es wurden die mittlere Anzahl der Arten je Aufnahmefläche sowie der ShannonIndex $\left(\mathrm{H}^{\prime}\right.$; basierend auf dem natürlichen Logarithmus, In), der Simpson's Index (D) und die Evenness (E; auf der Basis des Shannon-Index) berechnet (Tab. 1). Die Artenzahl wird durch die seltenen Arten am stärksten beeinflusst, während Simpson's Index (D) ein Maß für die Dominanz ist. Der Shannon-Index $\left(\mathrm{H}^{`}\right)$ nimmt hinsichtlich seiner Empfindlichkeit gegenüber seltenen Arten eine Mittelstellung zwischen Artenzahl und Simpson's Index ein. Die Evenness (E) ist ein Maß für die Ähnlichkeit der Abundanzen der verschiedenen Arten ( $\mathrm{McCu}$ ne $\&$ Grace 2002). Sind die Abundanzen der verschiedenen Arten identisch, ist die Evenness 1; sind sie völlig verschieden so ist die Evenness 0.

Der Anteil der jeweiligen Lebensformen (Zwergsträucher, Grasartige, Kräuter und Leguminosen) wurde aus den mittleren 
Tab. 1:

Übersicht über die Berechnungsmethoden von Shannon-Index ( $\left.\mathrm{H}^{\prime}\right)$, Simpson's Index (D) und Evenness (E).

\begin{tabular}{lll}
\hline Diversitäts-Index & Formel & Erklärung \\
\hline Shannon-Index & $\mathrm{H}^{`}=-\sum_{1=i}^{S} p_{i} \ln p_{i}$ & $\begin{array}{l}\text { Maß für den „Informations-Gehalt } \\
\text { einer Aufnahme. }\end{array}$ \\
Simpson's Index & $\mathrm{D}=1-\sum_{\mathrm{l}=i}^{S} p_{i}^{2}$ & $\begin{array}{l}\text { Wahrscheinlichkeit, dass zwei zufällig } \\
\text { ausgewählte Individuen verschiedenen } \\
\text { Arten angehören. }\end{array}$ \\
& $\mathrm{E}=\frac{H^{\prime}}{\ln S}=\frac{H^{\prime}}{H^{\prime} \max }$ & $\begin{array}{l}\text { \#Informations-Gehalt einer Aufnahme } \\
\text { im Vergleich zum maximal möglichen } \\
\text { Wert. }\end{array}$ \\
\hline
\end{tabular}

$S=$ Anzahl der Arten

$p_{i}=$ Anteil der Art $i$ an der Summe aller Abundanzen

$\ln =$ natürlicher Logarithmus

Deckungswerten der Schätzungen berechnet und in Relation zur Gesamtdeckung in Prozent angegeben. Für jede Aufnahme wurden außerdem die Zeigerwerte Feuchtezahl (F), Reaktionszahl (R) und Stickstoffzahl (N) nach Ellenberg et al. (1992) im Programm Juice 7.0 (L. Tichý, 1999-2010) berechnet. Die Berechnung der mittleren Zeigerwerte erfolgte ohne Gewichtung nach der Artmächtigkeit.

Die Erhebung von Bewirtschaftungsparametern führte Nagl (2009) mit Hilfe einer Befragung von Grundbesitzern und anderen Einheimischen durch. Dies geschah parallel zur Geländearbeit bzw. auf Grundlage detaillierter Gespräche, die im Anschluss an die vegetationskundlichen Untersuchungen im Jahr 2007 stattfanden. Dabei wurden genaue Auskünfte über die aktuelle und ehemalige Bewirtschaftung der Flächen eingeholt (Nagl 2009). Es wurden folgende Bewirtschaftungsklassen unterschieden: Zweischürige Talwiesen (intensiv, zwei Mal pro Jahr gemäht, starke Düngung), einschürige Bergmähder (mäßig intensiv, ein $\mathrm{Mal}$ pro Jahr gemäht, Düngung), halbschürige Bergmähder (extensiv, alle zwei Jahre gemäht, seltene Düngung), Brache jung (vor frühestens 10 Jahren aufgelassen), Brache alt (vor über 10 Jahren aufgelassen), Weiden (extensiv, Rinder und Pferde, nicht gedüngt).

Mit Ausnahme der Aufnahmen von Ellmauer (1995) wurde für jede Aufnahmefläche der pH-Wert ermittelt. Die Messung des $\mathrm{pH}$-Wertes erfolgte in einer 0,01 $\mathrm{M} \mathrm{CaCl}_{2}$-Lösung. Für jede Aufnah- 
mefläche wurde aus den Werten von drei Einzelproben das arithmetische Mittel berechnet.

\subsection{Klassifikation und Ordination}

Die Aufnahmen wurden im Programm TWINSPAN for Windows 2.3 (ter Braak \& Šmilauer 2004-2005) numerisch klassifiziert. Die TWINSPANTabelle wurde im Programm Juice 7.0 (L. Tichý, 1999-2010) in eine Stetigkeitstabelle umgewandelt. Die Stetigkeit einer Pflanzenart ist ihr Vorkommen in Prozent innerhalb einer bestimmten Gruppe von Aufnahmen. Nach Dierschke (1994) wurden folgende Stetigkeitsklassen unterschieden: $\mathrm{V}:>80 \%, \mathrm{IV}:>60$ $80 \%$, III: > 40-60\%, II: > 20-40\%, I: $\leq 20 \%$. Zusätzlich wurde eine Indikatorarten-Analyse (Dufrêne \& Legendre 1997) in PC-ORD 5.31 (MjM Software Design) durchgeführt. Die IndikatorWert-Analyse kombiniert die relative Abundanz und die relative Frequenz für die jeweiligen Pflanzenarten in einer Gruppe von Aufnahmen nach folgender Formel: Indikator-Wert $(\%)=\mathrm{A}_{\mathrm{ij}} \times \mathrm{B}_{\mathrm{ij}} \times 100 ; \mathrm{A}_{\mathrm{ij}}$ $=$ mittlere Abundanz der Art ${ }_{i}$ in den Aufnahmen der Gruppe ; $\mathrm{B}_{\mathrm{ij}}=$ relative Frequenz des Vorkommens der Art in den Aufnahmen der Gruppe (Dufrêne \& Legendre 1997). Der Indikator-Wert gibt den Anteil am maximal möglichen Wert in Prozent an (100\% für eine perfekte Indikatorart). Die Indikator-Werte wurden auf ihre Signifikanz hin getestet. Dazu diente ein Monte Carlo-Permutationstest mit 1999 Permutationen (McCune \& Grace 2002). Für die Identifizierung der Differenzialarten der Subassoziationen wurden in dieser Arbeit nur signifikante Indikator-Werte $(P \leq 0,05)$ mit $\geq 25 \%$ berücksichtigt.

Die Ordinationen wurden im Programm CANOCO 4.5 (ter Braak \& Šmilauer 1998) durchgeführt. Die DCA (Detrended Correspondence Analysis) ergab für den Gesamtdatensatz eine Gradientenlänge von > 4 Standardabweichungen (SD), daher wurde ein unimodales Modell zugrunde gelegt (ter Braak \& Šmilauer 1998). Für die Berechnung der DCA wurden zusätzlich supplementäre Variablen verwendet. Diese gingen nicht in die Berechnung der Achsen ein, jedoch wurden für sie anschließend mit den DCA-Achsen korreliert (Berechnung von Korrelationskoeffizienten) und passiv in das DCA-Diagramm eingeblendet. Die direkte Gradientenanalyse erfolgte mittels einer CCA (Canonical Correspondence Analysis), bei welcher die kanonischen Achsen von den Umweltvariablen gebildet werden (ter Braak \& Šmilauer 1998). Der Monte Carlo-Permutationstest (unrestricted, 1999 Permutationen) ergab, dass alle verwendeten Variablen signifikant waren $(P \leq 0,05)$ und daher wurden sie in das Modell mit aufgenommen. Der Datensatz wurde durch die Quadratwurzel transformiert, die seltenen Arten erhielten in der CCA-Analyse eine geringe- 
re Gewichtung. Die Ordinationsgrafiken wurden in CANODRAW 4.0 erstellt.

\subsection{Statistische Tests}

Die Unterschiede zwischen den Assoziationen und Subassoziationen hinsichtlich Umweltparameter, Diversitäts-Indices, Zeigerwerte und Anteile der Lebensformen wurden in PASW Statistics 18 (Polar Engineering and Consulting 1993-
2007) auf ihre Signifikanz hin geprüft. Dazu wurden univariate Varianzanalysen (ANOVA) und für Mehrgruppenvergleiche LSD post-hoc Tests verwendet. Die Werte wurden auf Normalverteilung (Kolmogorov-Smirnov-Test) und auf Gleichheit der Fehlervarianzen (LeveneTest) in PASW Statistics 18 getestet. In der weitaus überwiegenden Anzahl der Fälle waren die Voraussetzungen für die Anwendung der ANOVA gegeben. Aufgrund der hinreichend großen Stichpro-

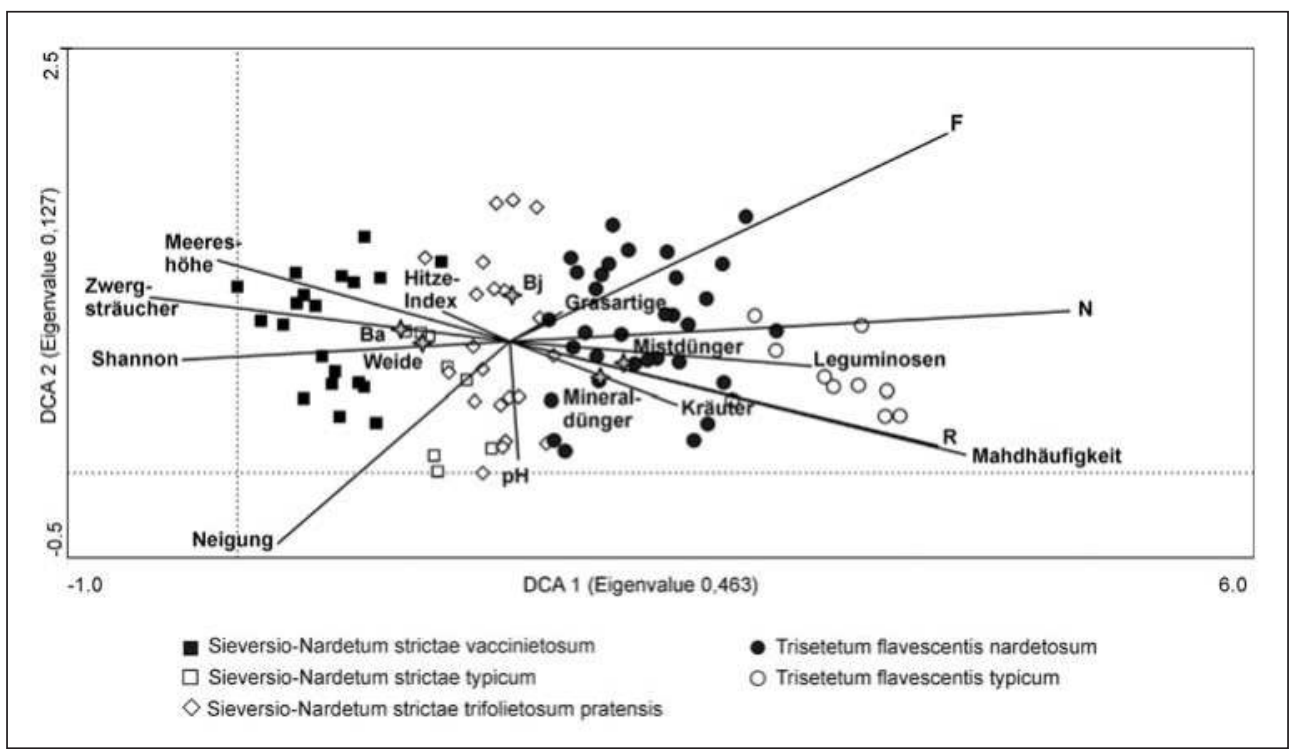

Abb. 2:

DCA der 91 Aufnahmen im Untersuchungsgebiet. Die verschiedenen Symbole stehen für die Zugehörigkeit der Aufnahmen zu den fünf Subassoziationen, welche durch die TWINSPAN-Analyse unterschieden worden sind. Folgende Variablen wurden als supplementäre Variablen passiv in das Diagramm eingeblendet: Meereshöhe, Neigung, pH-Wert des Bodens, Hitze-Index, Shannon-Index, Mahdhäufigkeit, Weide, Bj: Brache jung ( $\leq 10$ Jahre unbewirtschaftet), Ba: Brache alt (> 10 Jahre unbewirtschaftet), Mistdünger, Mineraldünger, Anteil der Zwergsträucher, Kräuter, Grasartigen und der Leguminosen (\%); Zeigerwerte: F: Feuchtezahl, R: Reaktionszahl, N: Stickstoffzahl. 
benanzahl wurden in den einzelnen Fällen der Verletzung dieser Voraussetzungen die Varianzanalysen trotzdem durchgeführt.

\section{Ergebnisse}

Die TWINSPAN-Analyse unterteilte im ersten Teilungsschritt den Gesamtdatensatz in eine Gruppe mit 49 und in eine Gruppe mit 42 Aufnahmen (Tab. A1). Die erste Gruppe kann als Sieversio-Nardetum strictae (Bürstlingsrasen) bezeichnet werden. Die zweite Gruppe repräsentiert das Trisetetum flavescentis (Goldhaferwiesen). Mit dem zweiten und dritten Teilungsschritt nach TWINSPAN ergaben sich insgesamt fünf Aufnahmegruppen (Tab. A1). Sowohl die indirekte (DCA, Abb. 2) als auch die direkte (CCA, Abb. 3) Ordination bestätigt diese Auftrennung.

Die CCA-Analyse zeigte, dass vor allem die Bewirtschaftung einen wesentlichen Einfluss auf die Ausbildung der Pflanzengesellschaften hatte (Abb. 3). Die Mahdhäufigkeit wies die höchste Korrelation mit der ersten CCA-Achse auf $(+0,7790)$

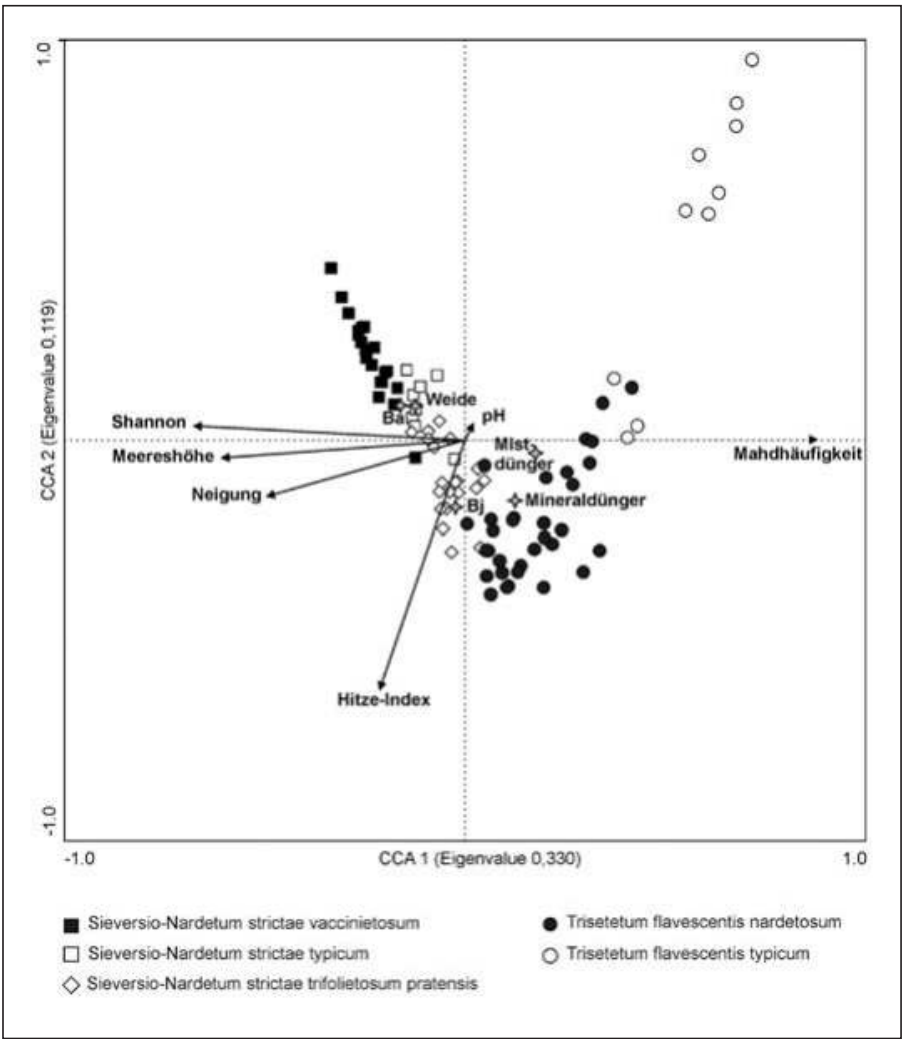

Abb. 3:

CCA der 91 Aufnahmen im Untersuchungsgebiet. Die verschiedenen Symbole für die fünf Subassoziationen wurden anhand der Ergebnisse der TWINSPAN-Analyse den Aufnahmen zugeordnet. Folgende Umweltvariablen gingen in die Berechnung der CCA-Achsen ein: Meereshöhe, Neigung, Hitze-Index, pH-Wert des Bodens, Shannon-Index, Mahdhäufigkeit, Mistdünger, Mineraldünger, Weide, Bj: Brache jung ( $\leq 10$ Jahre unbewirtschaftet), Ba: Brache alt (> 10 Jahre unbewirtschaftet). 
Tab. 2:

Mittelwerte \pm Standardfehler der Umweltparameter, Diversitäts-Indices, Zeigerwerte und Anteile der Lebensformen (\%) im Sieversio-Nardetum strictae und im Trisetetum flavescentis; $\mathrm{n}=$ Stichprobenumfang. Signifikante Unterschiede $(P \leq 0,05)$ zwischen den Assoziationen aufgrund der univariaten Varianzanalysen (ANOVA) sind fett dargestellt.

\begin{tabular}{|c|c|c|c|c|}
\hline \multirow{2}{*}{ Abhängige Variable } & \multicolumn{2}{|c|}{ Sieversio-Nardetum strictae } & \multicolumn{2}{|c|}{ Trisetetum flavescentis } \\
\hline & $\mathrm{n}$ & Wert & $\mathrm{n}$ & Wert \\
\hline Meereshöhe (m) & 49 & $2019 \pm 13$ & 42 & $1946 \pm 14$ \\
\hline Neigung $\left(^{\circ}\right)$ & 49 & $24,0 \pm 1,7$ & 39 & $16,3 \pm 1,9$ \\
\hline Hitze-Index & 47 & $106 \pm 5$ & 30 & $130 \pm 7$ \\
\hline pH-Wert Boden & 49 & $4,17 \pm 0,04$ & 39 & $4,46 \pm 0,05$ \\
\hline Artenzahl & 49 & $38,1 \pm 1,0$ & 42 & $23,4 \pm 1,1$ \\
\hline Shannon-Index & 49 & $2,93 \pm 0,04$ & 42 & $2,55 \pm 0,05$ \\
\hline Simpson's Index & 49 & $0,89 \pm 0,01$ & 42 & $0,87 \pm 0,01$ \\
\hline Evenness (Shannon) & 49 & $0,80 \pm 0,01$ & 42 & $0,82 \pm 0,01$ \\
\hline Feuchtezahl (F) & 49 & $4,83 \pm 0,05$ & 42 & $5,43 \pm 0,05$ \\
\hline Reaktionszahl (R) & 49 & $4,61 \pm 0,08$ & 42 & $5,43 \pm 0,08$ \\
\hline Stickstoffzahl (N) & 49 & $3,11 \pm 0,09$ & 42 & $4,79 \pm 4,60$ \\
\hline Zwergsträucher (\%) & 49 & $10,9 \pm 1,8$ & 42 & $0,0 \pm 0,0$ \\
\hline Grasartige (\%) & 49 & $39,7 \pm 2,1$ & 42 & $45,1 \pm 2,2$ \\
\hline Kräuter (\%) & 49 & $44,0 \pm 2,2$ & 42 & $42,6 \pm 2,3$ \\
\hline Leguminosen (\%) & 49 & $5,4 \pm 1,1$ & 42 & $12,2 \pm 1,2$ \\
\hline
\end{tabular}

gefolgt von Mistdüngung (+0,5733). Shannon-Index $(-0,6003)$, Meereshöhe $(-0,5396)$, Brache alt $(-0,4516)$ und Neigung $(-0,4394)$ waren hingegen deutlich negativ mit der ersten CCA-Achse korreliert. Das zeigt, dass die Flächen in höheren Lagen extensiver bewirtschaftet werden oder inzwischen aufgelassen wurden. Demgegenüber steigt die Arten- vielfalt, ausgedrückt durch den ShannonIndex, mit zunehmender Meereshöhe und abnehmender Bewirtschaftungsintensität an. Die Beweidung zeigte eine deutlich niedrigere negative Korrelation mit der ersten CCA-Achse (-0,3269). Der Hitze-Index $(-0,4932)$ war am deutlichsten negativ mit der zweiten CCAAchse korreliert gefolgt von Mineraldün- 
ger $(-0,3646)$. Der pH-Wert war für die Gruppenbildung nicht relevant.

Sowohl die meisten Umweltparameter als auch Artenzahl, Shannon-Index und alle Zeigerwerte unterschieden sich signifikant zwischen dem Sieversio-Nardetum strictae und dem Trisetetum flavescentis. Das Sieversio-Nardetum strictae ist in größerer Meereshöhe ausgebildet, bewächst steilere Hänge und der Boden weist einen niedrigeren $\mathrm{pH}$-Wert auf. Die Hänge sind vorwiegend in südliche bis südöstliche Richtung und damit für das Pflanzenwachstum günstiger exponiert als beim Trisetetum flavescentis. Die mittlere Anzahl der Arten und der Shannon-Index sind im Sieversio-Nardetum strictae signifikant höher als im Trisetetum flavescentis (Tab. 2). Besonders deutlich sind die Unterschiede hinsichtlich Feuchtezahl (F), Reaktionszahl (R) und Stickstoffzahl (N), welche im Trisetetum flavescentis höher liegen. So ist beispielsweise die Stickstoffzahl um mehr als eineinhalb Zeigerwert-Klassen erhöht. Hinsichtlich der verschiedenen Lebensformen weist das Sieversio-Nardetum strictae einen signifikant höheren Anteil an Zwergsträuchern auf, während im Trisetetum flavescentis der Anteil der Leguminosen signifikant höher ausfällt (Tab. 2).

\subsection{Sieversio-Nardetum strictae Lüdi 1948 (Bürstlingsrasen)}

Das Sieversio-Nardetum strictae hebt sich durch eine Reihe von Differenzialarten vom Trisetetum flavescentis ab. Die Arten mit der höchsten Stetigkeit sind Carex sempervirens, Nardus stricta, Lotus corniculatus, Luzula multiflora, Silene nutans, Brizia media und Carlina acaulis (D1-D3, Táb. A1). Diese Gesellschaft wird der $\mathrm{Kl}$ csse Caricetea curvulae Br.-Bl. 1948 (Subalpin-alpine Sauerbodenrasen der mittel-südeuropäischen Hochgebirge) zugeordnet (Grabherr 1993). Die wichtigsten Charakterarten der Klasse sind Potentilla aurea, Avenula versicolor, Gentiana acaulis und Trifolium alpinum (Tab. A1). Innerhalb dieser Klasse gehört die Gruppe in die Ordnung Festucetalia spadiceae Barbero 1970 em. Grabherr (Bodensaure Wildheumähder, Weiden und Lawinarwiesen, Grabherr 1993). Das wird durch die Charakterarten der Ordnung belegt: so z.B. Phyteuma betonicifolium, Campanula barbata und Geum montanum (Tab. A1). Ajuga pyramidalis und Pseudorchis albida sprechen für die Zuordnung zum Verband Nardion strictae Br.-Bl. 1926 (Bürstlingsrasen der nemoralen Hochgebirge Europas) (Tab. A1). Schließlich wurde diese Gruppe der Assoziation Sieversio-Nardetum strictae (Subalpinalpine Bürstlingsweiden und -mähder) zugeordnet. 
Tab. 3:

Die signifikanten Indikator-Werte (\%) für die jeweiligen Subassoziationen des Sieversio-Nardetum strictae und des Trisetetum flavescentis. Die Indikatorwerte müssen $\geq 25 \%$ erreichen und sie müssen mindestens doppelt so hoch sein, als in einer der anderen Subassoziationen. Die höchsten Indikatorwerte in einer Subassoziation sind fett dargestellt. Folgende Subassoziationen wurden unterschieden:

1: Sieversio-Nardetum strictae vaccinietosum

2: Sieversio-Nardetum strictae typicum

3: Sieversio-Nardetum strictae trifolietosum pratensis

4: Trisetetum flavescentis nardetosum

5: Trisetetum flavescentis typicum

\begin{tabular}{|c|c|c|c|c|c|}
\hline \multirow{2}{*}{ Indikator-Werte (\%) } & \multicolumn{5}{|c|}{ Subassoziation Nr. } \\
\hline & 1 & 2 & 3 & 4 & 5 \\
\hline Vaccinium vitis-idaea & 63 & 4 & 1 & 0 & 0 \\
\hline Calluna vulgaris & 62 & 11 & 2 & 0 & 0 \\
\hline Pedicularis tuberosa & 60 & 0 & 0 & 0 & 0 \\
\hline Gymnadenia conopsea & 52 & 0 & 1 & 0 & 0 \\
\hline Avenula versicolor & 51 & 0 & 4 & 0 & 0 \\
\hline Campanula barbata & 50 & 7 & 15 & 0 & 0 \\
\hline Vaccinium gaultherioides & 49 & 4 & 1 & 0 & 0 \\
\hline Gentiana acaulis & 47 & 10 & 2 & 0 & 0 \\
\hline Trifolium alpinum & 45 & 5 & 0 & 0 & 0 \\
\hline Vaccinium myrtillus & 45 & 18 & 7 & 0 & 0 \\
\hline Potentilla erecta & 42 & 13 & 10 & 0 & 0 \\
\hline Antennaria dioica & 40 & 2 & 0 & 0 & 0 \\
\hline Arnica montana & 40 & 2 & 0 & 0 & 0 \\
\hline Avenella flexuosa & 39 & 15 & 4 & 0 & 0 \\
\hline Scabiosa columbaria & 35 & 0 & 0 & 0 & 0 \\
\hline Trifolium montanum & 32 & 0 & 3 & 0 & 0 \\
\hline Hieracium hoppeanum & 32 & 0 & 4 & 0 & 0 \\
\hline Juncus trifidus & 30 & 2 & 4 & 0 & 0 \\
\hline Helianthemum grandiflorum & 25 & 3 & 2 & 0 & 0 \\
\hline Hieracium pilosella & 10 & 68 & 1 & 0 & 0 \\
\hline Thymus pulegioides & 14 & 47 & 1 & 0 & 0 \\
\hline Veronica officinalis & 0 & 38 & 0 & 0 & 0 \\
\hline Potentilla grandiflora & 0 & 28 & 2 & 0 & 0 \\
\hline
\end{tabular}




\begin{tabular}{lccccc} 
Geranium sylvaticum & 0 & 8 & 16 & $\mathbf{3 6}$ & 4 \\
Crepis aurea & 0 & 0 & 6 & $\mathbf{3 5}$ & 0 \\
Poa alpina & 0 & 9 & 3 & $\mathbf{3 0}$ & 1 \\
Phleum pratensis & 0 & 0 & 0 & 1 & $\mathbf{8 3}$ \\
Taraxacum Sect. Ruderalia & 0 & 0 & 0 & 7 & $\mathbf{8 0}$ \\
Poa trivialis & 0 & 0 & 0 & 14 & $\mathbf{5 7}$ \\
Carum carvi & 0 & 1 & 0 & 1 & $\mathbf{5 5}$ \\
Poa pratensis & 0 & 1 & 0 & 3 & $\mathbf{5 5}$ \\
Trifolium repens & 0 & 7 & 3 & 11 & $\mathbf{5 3}$ \\
Elymus repens & 0 & 0 & 0 & 0 & $\mathbf{5 0}$ \\
Trifolium pratense ssp. pratense & 0 & 0 & 0 & 3 & $\mathbf{4 0}$ \\
\hline
\end{tabular}

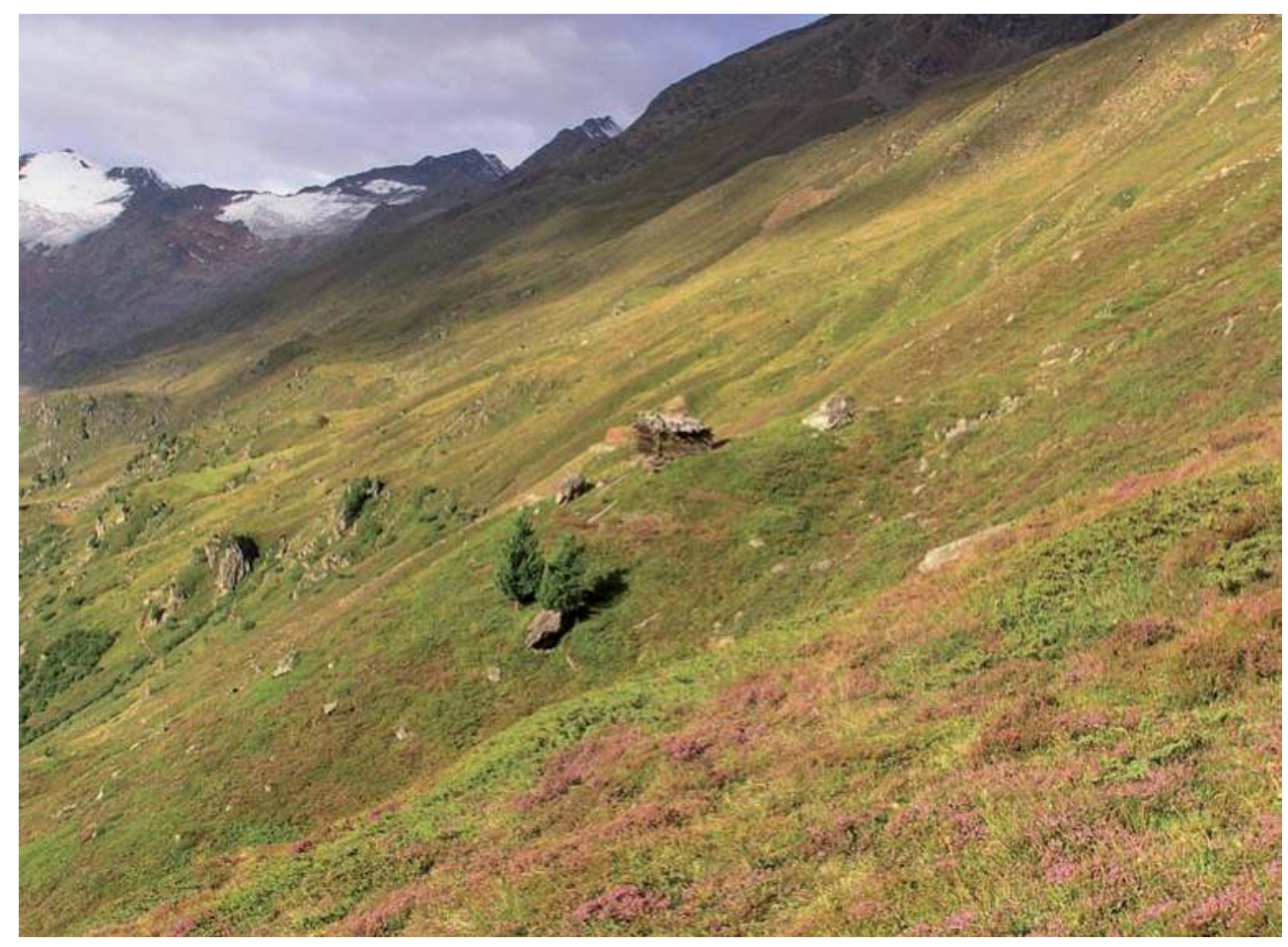

Abb. 4:

Das Sieversio-Nardetum strictae vaccinietosum weist einen hohen Anteil an Zwergsträuchern, wie zum Beispiel Calluna vulgaris im Vordergrund, auf. (Foto: Fabian Nagl) 
Tab. 4:

Mittelwerte \pm Standardfehler der Umweltparameter, Diversitäts-Indices, Zeigerwerte und Anteile der Lebensformen (\%) in den fünf Subassoziationen, die beim Sieversio-Nardetum strictae und Trisetetum flavescentis unterschieden wurden. Verschiedene hochgestellte Buchstaben bedeuten einen signifikanten Unterschied ( $P £ \leq 0,05$; LSD Post-hoc Test). Folgende Subassoziationen wurden unterschieden:

1: Sieversio-Nardetum strictae vaccinietosum

2: Sieversio-Nardetum strictae typicum

3: Sieversio-Nardetum strictae trifolietosum pratensis

4: Trisetetum flavescentis nardetosum

5: Trisetetum flavescentis typicum

\begin{tabular}{lccccc}
\hline Assoziationen & \multicolumn{3}{c}{ Sieversio-Nardetum strictae } & \multicolumn{2}{c}{ Trisetetum flavescentis } \\
Subassoziation Nr. & 1 & 2 & 3 & 4 & 5 \\
\hline Meereshöhe (m) & $2056 \pm 19^{\mathrm{a}}$ & $1978 \pm 30^{\mathrm{b}}$ & $1998 \pm 18^{\mathrm{b}}$ & $1966 \pm 15^{\mathrm{b}}$ & $1879 \pm 26^{\mathrm{c}}$ \\
Neigung $\left(^{\circ}\right)$ & $23,0 \pm 2,4^{\mathrm{a}}$ & $27,5 \pm 3,8^{\mathrm{a}}$ & $23,7 \pm 2,4^{\mathrm{a}}$ & $20,7 \pm 2,0^{\mathrm{a}}$ & $3,6 \pm 3,4^{\mathrm{b}}$ \\
Hitze-Index & $99 \pm 9^{\mathrm{a}}$ & $103 \pm 13^{\mathrm{ab}}$ & $115 \pm 8^{\mathrm{ab}}$ & $132 \pm 7^{\mathrm{b}}$ & $108 \pm 22^{\mathrm{ab}}$ \\
pH-Wert Boden & $4,11 \pm 0,06^{\mathrm{a}}$ & $4,16 \pm 0,10^{\mathrm{a}}$ & $4,24 \pm 0,06^{\mathrm{a}}$ & $4,41 \pm 0,05^{\mathrm{b}}$ & $4,61 \pm 0,09^{\mathrm{c}}$ \\
\hline Artenzahl & $40,2 \pm 1,5^{\mathrm{a}}$ & $39,0 \pm 2,4^{\mathrm{ab}}$ & $35,8 \pm 1,5^{\mathrm{b}}$ & $25,7 \pm 1,2^{\mathrm{c}}$ & $16,0 \pm 2,1^{\mathrm{d}}$ \\
Shannon-Index & $2,95 \pm 0,07^{\mathrm{a}}$ & $2,90 \pm 0,10^{\mathrm{a}}$ & $2,92 \pm 0,06^{\mathrm{a}}$ & $2,63 \pm 0,05^{\mathrm{b}}$ & $2,30 \pm 0,09^{\mathrm{c}}$ \\
Simpson's Index & $0,88 \pm 0,01^{\mathrm{ab}}$ & $0,88 \pm 0,02^{\mathrm{ab}}$ & $0,90 \pm 0,01^{\mathrm{a}}$ & $0,88 \pm 0,01^{\mathrm{ab}}$ & $0,85 \pm 0,01^{\mathrm{b}}$ \\
Evenness (Shannon) & $0,80 \pm 0,01^{\mathrm{a}}$ & $0,80 \pm 0,02^{\mathrm{a}}$ & $0,82 \pm 0,01^{\mathrm{a}}$ & $0,81 \pm 0,01^{\mathrm{a}}$ & $0,83 \pm 0,02^{\mathrm{a}}$ \\
\hline Feuchtezahl (F) & $4,68 \pm 0,07^{\mathrm{a}}$ & $4,66 \pm 0,11^{\mathrm{a}}$ & $5,03 \pm 0,07^{\mathrm{b}}$ & $5,38 \pm 0,05^{\mathrm{c}}$ & $5,61 \pm 0,10^{\mathrm{d}}$ \\
Reaktionszahl (R) & $4,26 \pm 0,10^{\mathrm{a}}$ & $4,58 \pm 0,17^{\mathrm{a}}$ & $4,97 \pm 0,10^{\mathrm{b}}$ & $5,28 \pm 0,08^{\mathrm{c}}$ & $5,89 \pm 0,15^{\mathrm{d}}$ \\
Stickstoffzahl (N) & $2,69 \pm 0,10^{\mathrm{a}}$ & $3,12 \pm 0,15^{\mathrm{b}}$ & $3,51 \pm 0,09^{\mathrm{c}}$ & $4,51 \pm 0,08^{\mathrm{d}}$ & $5,67 \pm 0,14^{\mathrm{c}}$ \\
\hline Zwergsträucher (\%) & $24,0 \pm 2,3^{\mathrm{a}}$ & $3,5 \pm 3,6^{\mathrm{b}}$ & $1,3 \pm 2,2^{\mathrm{b}}$ & $0,0 \pm 0,0^{\mathrm{b}}$ & $0,0 \pm 0,0^{\mathrm{b}}$ \\
Grasartige (\%) & $33,7 \pm 3,2^{\mathrm{a}}$ & $41,5 \pm 5,1^{\mathrm{ab}}$ & $44,7 \pm 3,1^{\mathrm{b}}$ & $44,8 \pm 2,5^{\mathrm{b}}$ & $46,3 \pm 4,6^{\mathrm{b}}$ \\
Kräuter (\%) & $38,8 \pm 3,4^{\mathrm{a}}$ & $49,9 \pm 5,4^{\mathrm{a}}$ & $46,7 \pm 3,3^{\mathrm{a}}$ & $43,9 \pm 2,7^{\mathrm{a}}$ & $38,7 \pm 4,8^{\mathrm{a}}$ \\
Leguminosen (\%) & $3,6 \pm 1,7^{\mathrm{a}}$ & $5,1 \pm 2,7^{\mathrm{ab}}$ & $7,2 \pm 1,6^{\mathrm{abc}}$ & $11,4 \pm 1,3^{\mathrm{cd}}$ & $15,1 \pm 2,4^{\mathrm{d}}$ \\
\hline
\end{tabular}

Das Sieversio-Nardetum strictae wurde in drei Subassoziationen unterteilt: vaccinietosum, typicum und trifolietosum pratensis (Tab. A1). Die Subassoziation Sieversio-Nardetum strictae vaccinietosum Hartl 1963 ist durch eine Reihe von
Differenzialarten gut belegt (D1, Tab. A1), dazu zählen die hochsteten Arten Vaccinium vitis-idaea, Potentilla erecta und Vaccinium gaultherioides. Die Indikatorwert-Analyse wies 19 Arten aus, welche diese Subassoziation eindeutig cha- 
rakterisieren (Tab. 3), wobei die höchsten Indikatorwerte von den Zwergsträuchern Vaccinium vitis-idaea und Calluna vulgaris erreicht werden (Abb. 4). Im Vergleich zu den anderen Subassoziationen gedeiht das Sieversio-Nardetum strictae vaccinietosum mit durchschnittlich $2056 \mathrm{~m}$ Meereshöhe in den höchsten Lagen des Untersuchungsgebietes (Tab. 4). Hier wurde der geringste Hitze-Index (99) und damit die für das Pflanzenwachstum günstigste Exposition ermittelt. Diese Subassoziation weist die höchste mittlere Artenzahl und den höchsten Shannon-Index auf (40 bzw. 2,95, Tab. 4). Die Reaktions- (R) und Stickstoffzahl (N) haben die niedrigsten Werte von allen Subassoziationen (Tab. 4). Der Anteil der Zwergsträucher ist mit $24 \%$ um ein Vielfaches höher als in allen anderen Subassoziationen des Sieversio-Nardetum strictae (Tab. 4). Dementsprechend ist der Anteil der Leguminosen mit durchschnittlich nur 3,6 \% am geringsten. $55 \%$ der Aufnahmeflächen sind alte und $10 \%$ sind junge Brachen. $35 \%$ werden aktuell vorwiegend beweidet (Tab. A1).

Das Sieversio-Nardetum strictae typicum Br.-Bl. 1949 ist durch die Differenzialarten Hieracium pilosella und Veronica officinalis unterschieden (D2, Tab. A1). Die Indikatorwert-Analyse wies zwei zusätzliche Arten, nämlich Thymus pulegioides und Potentilla grandiflora, aus (Tab. 3). Diese Subassoziation gedeiht mit durchschnittlich $1978 \mathrm{~m}$ in signifikant geringerer Meereshöhe als das Sieversio-
Nardetum strictae vaccinietosum (Tab. 4). Die Hänge sind hier im Durchschnitt jedoch am steilsten $\left(27,5^{\circ}\right.$ geneigt). Der Artenreichtum unterscheidet sich nicht signifikant von den anderen Subassoziationen des Sieversio-Nardetum strictae (Tab. 4). Die Stickstoffzahl (N) ist jedoch signifikant höher als in der Subassoziation vaccinietosum (Tab. 4). Der Anteil der Zwergsträucher ist gering (nur 3,5 \% der Gesamtdeckung); besonders hoch ist im Vergleich dazu der Anteil der Kräuter (durchschnittlich 49,5 \%; Tab. 4). Meistens handelt es sich um Weiden $(62,5 \%)$, seltener sind es alte Brachen (25,0 \%) oder einschürige Mähder (12,5\%). Die gemähten Flächen werden mit Mist und Mineraldünger gedüngt (Tab. A1). Das Sieversio-Nardetum strictae trifolietosum pratensis Br-Bl. 1949 vermittelt durch Phleum rhaeticum (Begleiter, Tab. A1) bereits zu den stärker nährstoffversorgten Mähwiesen des Untersuchungsgebietes (Abb. 5). Hier gibt es keine eigene Differenzialartengruppe. Auch die Indikatorwert-Analyse erbrachte keine Arten, die diese Subassoziation eindeutig charakterisieren (Tab. 3). Die Gesellschaft gedeiht in etwa in gleicher mittlerer Meereshöhe wie das Sieversio-Nardetum strictae typicum (1998 m, Tab. 4). Die mittlere Artenzahl pro Aufnahmefläche geht auf 36 zurück (Tab. 4). Feuchte- (F), Reaktions- (R) und Stickstoffzahl (N) sind signifikant höher als in den beiden anderen Subassoziationen des Sieversio- 


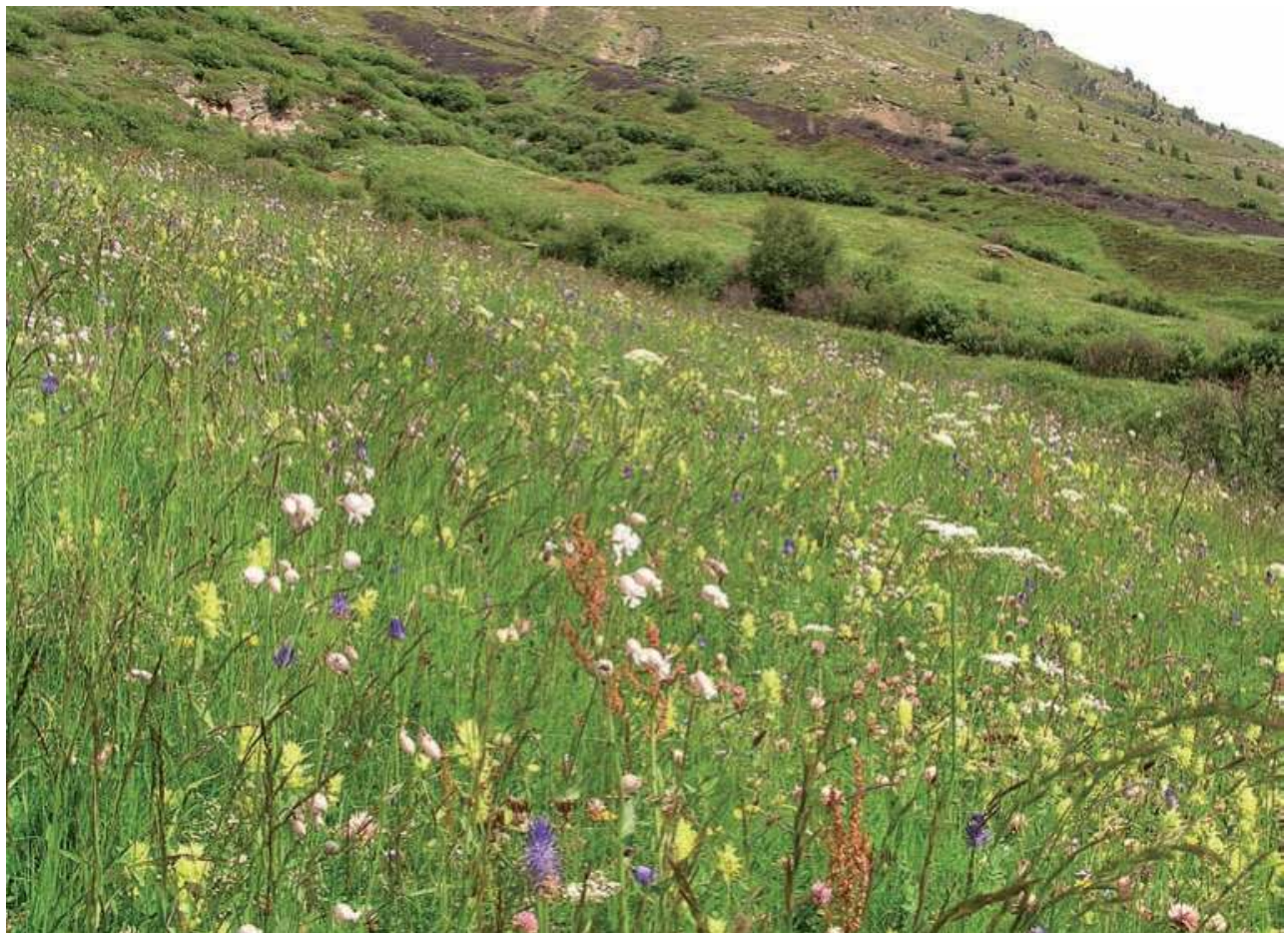

Abb. 5:

Das Sieversio-Nardetum strictae trifolietosum pratensis gedeiht üppiger und zeigt Anklänge an nährstoffreichere Bestände. (Foto: Fabian Nagl)

Nardetum strictae (Tab. 4). Der Anteil der Zwergsträucher sinkt weiter auf nur noch $1,3 \%$ (Tab. 4). Der durchschnittliche Anteil der Leguminosen steigt leicht auf $7,2 \%$. Nur noch ein Drittel der Flächen wird beweidet, während der Anteil der halb- und einschürigen Mähder ansteigt (Tab. A1). Fast alle dieser gemähten Flächen werden im Herbst mit Schafen oder Rindern nachbeweidet. Einige junge und alte Brachen kommen vor. Der Anteil der mit Mist und mit Mineraldünger behandelten Flächen steigt weiter an $(28,6$ bzw. 23,8 \%, Tab. A1).
Die subalpinen Borstgrasrasen in Obergurgl zeigen eine gute Übereinstimmung mit der Originalbeschreibung von Lüdi (1948) aus den Berner Alpen. Die Bestände dort weisen zahlreiche Elemente der Frischwiesen auf und ähneln daher besonders der in der vorliegenden Arbeit beschriebenen Subassoziation trifolietosum pratensis. Ähnlichkeiten finden sich ebenfalls zur Studie von Peppler-Lisbach \& Petersen (2001) in den Bayrischen Alpen. Auch diese Autoren unterschieden die Subassoziationen Sieversio-Nardetum strictae typicum und trifolietosum pra- 
tensis. Weitere Bezüge bestehen zum Aveno-Nardetum Oberd. 1959 - (typische Ausbildung) am Tappenkar in den Radstädter Tauern (Heiselmayer 1982), auch wenn die Anzahl der gemeinsam auftretenden konstanten Arten eher gering ist. Übereinstimmungen gibt es weiters zwischen der in Obergurgl beschriebenen typischen Subassoziation und den höher gelegenen Weideflächen auf der Kompardellalm in der Samnaun-Gruppe (Wagner 1965). Gute Übereinstimmungen gibt es schließlich mit dem Sieversio-Nardetum strictae, welches Unterluggauer (2003) im benachbarten Venter- und Rofental untersuchte. Nährstoffliebende Arten sind in Obergurgl jedoch weit wichtiger, während in Vent der Anteil an Magerkeitszeiger höher ist.

\subsection{Trisetetum flavescentis Rübel 1911 (Goldhaferwiesen)}

Das Trisetetum flavescentis weist nur wenige stete Differenzialarten auf wie Phleum pratense, Taraxacum Sect. Ruderalia, Poa trivialis, Trifolium repens, Poa pratensis und Carum carvi (D5, Tab. A1). Es unterscheidet sich vor allem durch das Zurücktreten bzw. Ausfallen der für das SieversioNardetum strictae typischen Arten (Tab. A1). Das Trisetetum flavescentis wird in die Klasse Molinio-Arrhenatheretea R. Tx. 1937 em. R. Tx. 1970 gestellt (Ellmauer \& Mucina 1993). Dafür sprechen zahlreiche Klassen-Charakterarten wie Alchemil- la vuglaris agg., Achillea millefolium agg., Leontodon hispidus, Ranunculus acris und Deschampsia cespitosa (Tab. A1). Die Ordnung Poo apinae-Trisetetalia Ellmauer et Mucina 1993 (Almwiesen und -weiden, Ellmauer \& Mucina 1993) ist durch die Charakterarten Agrostis capillaris, Rumex alpestris, Campanula scheuchzeri und Poa alpina belegt (Tab. A1). Die Verbandscharakterarten des Polygono-Trisetion Br.-Bl. et R. Tx. ex Marschall 1947 nom. inv. (Gebirgs-Goldhafer-Wiesen) Trollius europaeus, Crocus albiflorus und Viola tricolor sind zwar nur spärlich vorhanden, doch es gedeihen zahlreiche dominante und konstante Begleiter der Assoziation Trisetetum flavescentis wie etwa Festuca nigrescens, Trifolium pratense ssp. nivale und Geranium sylvaticum. Trisetum flavescens (Goldhafer) tritt hingegen nur in geringer Stetigkeit auf (Tab A1).

Das Trisetetum flavescentis gliedert sich in die Subassoziationen nardetosum und typicum (Tab. A1). Die Subassoziation nardetosum (Abb. 6) hat zahlreiche hochstete Arten mit dem Sieversio-Nardetum strictae gemeinsam (Begleiter, Tab. A1), verfügt aber über keine eigene Differenzialartengruppe. Die Indikatorarten-Analyse belegt jedoch die Differenzierung der Subassoziation mit den Arten Geranium sylvaticum, Crepis aurea und Poa alpina (Tab. 3). Sie gedeiht im Mittel auf 1966 $\mathrm{m}$ Meereshöhe. Diese Flächen weisen unter allen Subassoziationen den höchsten Hitze-Index (im Durchschnitt 132) und damit die für das Pflanzenwachstum un- 


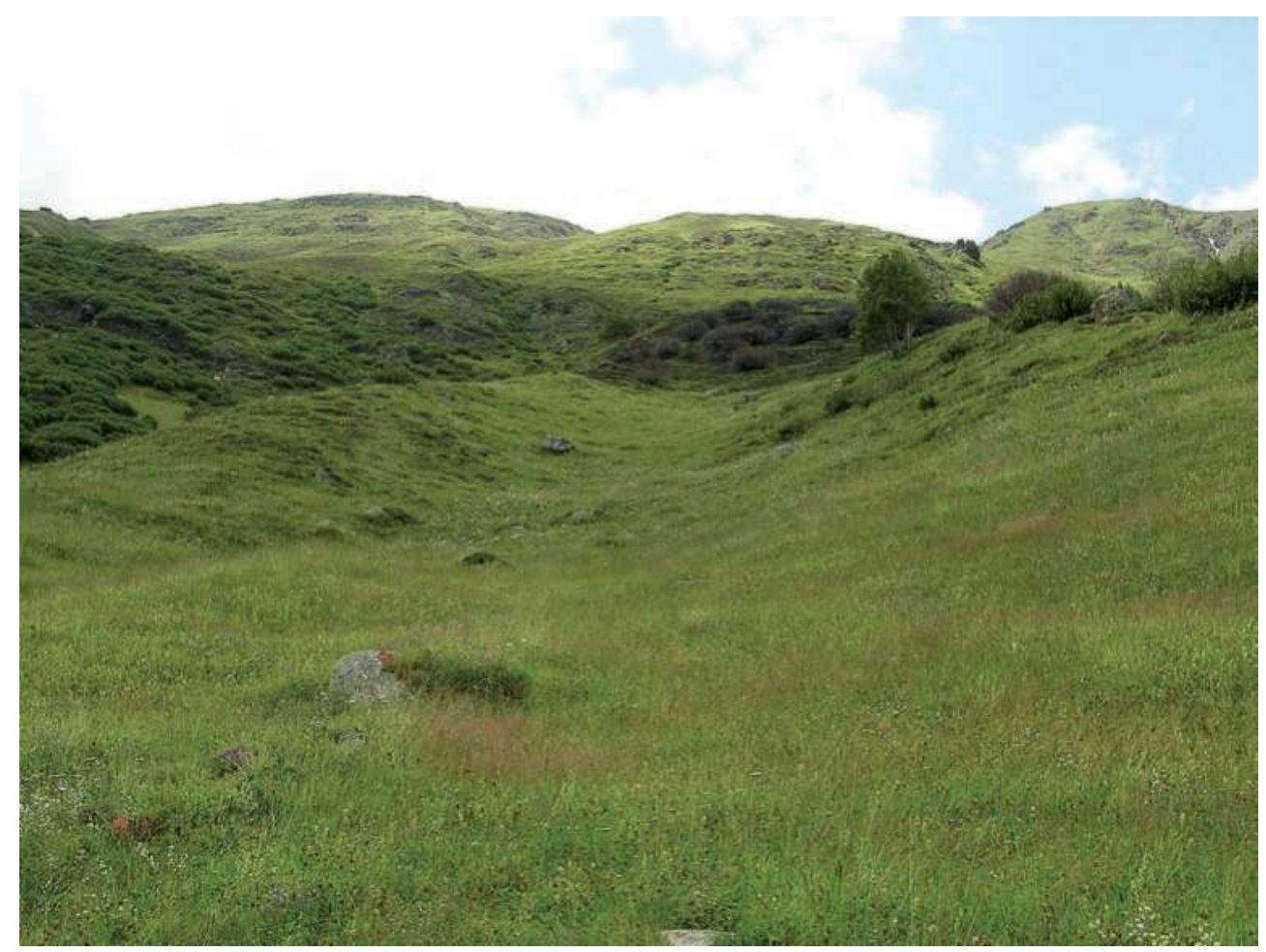

Abb. 6:

Das Trisetetum flavescentis nardetosum hat zahlreiche Arten mit dem Sieversio-Nardetum strictae gemeinsam. (Foto: Fabian Nagl)

günstigste Exposition auf (Tab. 4). Die mittlere Artenzahl und der mittlere Shannon-Index nehmen im Vergleich zum Sieversio-Nardetum strictae signifikant ab und betragen im Durchschnitt nur noch 26 Arten bzw. 2,63 pro Aufnahmefläche (Tab. 4). Feuchte-, Reaktions- und Stickstoffzahl sind signifikant höher als in den Subassoziationen des Sieversio-Nardetum strictae (Tab. 4). Der Anteil der Leguminosen steigt gegenüber dem SieversioNardetum strictae deutlich an und beträgt nun im Mittel 11,3\%, Zwergsträucher fehlen (Tab. 4). Mehr als drei Viertel der Flächen werden aktuell einmal pro Jahr gemäht und überwiegend mit Mist, oft aber auch zusätzlich mit Mineraldünger gedüngt (Tab. A1). Vor allem der Anteil der alten Brachen sinkt deutlich gegenüber dem Sieversio-Nardetum strictae, während der Anteil der jungen Brachen hier am höchsten ausfällt (Tab. A1).

Das Trisetetum flavescentis typicum (Abb. 7) hebt sich durch eine umfangreiche Differenzialartengruppe gegenüber der Subassoziation nardetosum $a b$ 
Kapitel 1 I Subalpine Wiesen und Weiden - die Kulturlandschaften der subalpinen Stufe

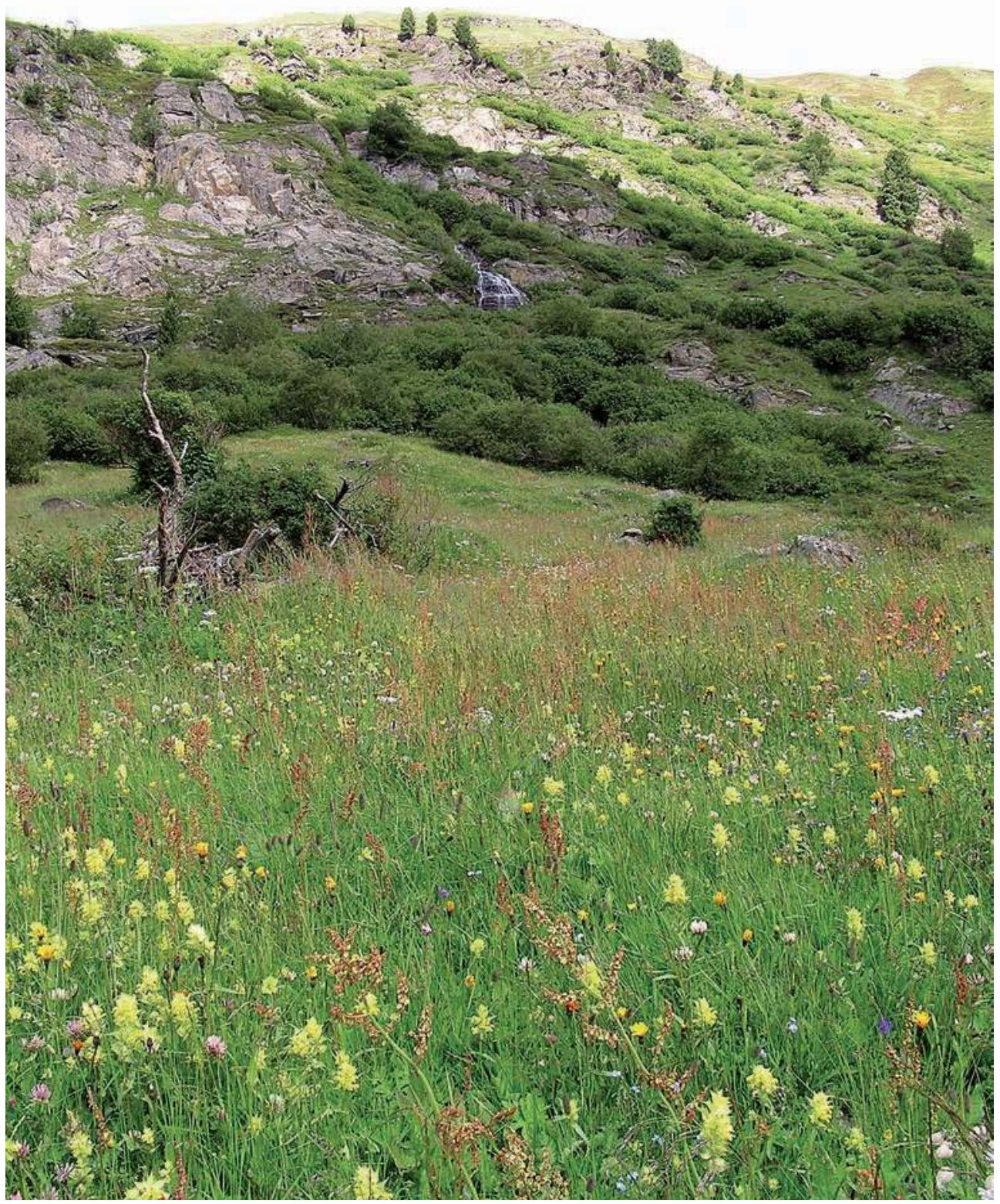

Abb. 7:

Das Trisetetum flavescentis typicum gedeiht am Talboden, wird intensiv bewirtschaftet und hat einen auffallend niedrigen Artenreichtum. (Foto: Fabian Nagl) 
(D5, Tab. A1). Die wichtigsten davon sind Phleum pratense, Taraxacum Sect. Ruderalia, Poa trivialis, Trifolium repens, Poa pratensis und Carum carvi. Die Indikatorwert-Analyse ermittelte acht Arten, welche für diese Gesellschaft typisch sind und mit den Differenzialarten fast gänzlich übereinstimmen (Tab. 3). Von allen Subassoziationen gedeiht das Trisetetum flavescentis typicum in den niedrigsten Lagen des Untersuchungsgebietes (1879 $\mathrm{m})$, wo die Hänge nicht bis nur wenig geneigt sind. Der pH-Wert des Bodens weist hier den höchsten Wert im Vergleich zu den anderen Subassoziationen auf (Tab. 4). Die durchschnittliche Artenzahl und der mittlere Shannon-Index (16 bzw. 2,30) sind signifikant niedriger als in allen anderen Subassoziationen, vor allem im Vergleich zu jenen des Sieversio-Nardetum strictae. Gegenüber allen anderen Subassoziationen sind Feuchte-, Reaktions- und Stickstoffzahl signifikant höher (Tab. 4). Auch der Anteil der Leguminosen ist mit durchschnittlich 15,1\% am höchsten. Alle Bestände werden gemäht, 70 \% zweimal im Jahr, $30 \%$ einmal. Alle Flächen werden mit Mist gedüngt, $30 \%$ davon zusätzlich auch mit Mineraldünger (Tab. A1). Diese Goldhaferwiesen werden zudem im Herbst mit Rindern und/oder Schafen nachbeweidet. Während der Anteil der Nachweide in der Subassoziation nardetosum nur etwa $40 \%$ beträgt, liegt er bei der Subassoziation typicum doppelt so hoch (Nagl 2009).
Im Vergleich zu den in der Literatur beschriebenen Goldhaferwiesen unterscheidet sich das Trisetetum flavescentis des Untersuchungsgebietes durch seinen auffallend geringen Artenreichtum. Selbst im Vergleich zum Trisetum flavescentis im benachbarten Venter- und Rofental (Unterluggauer 2003, Mayer et al. 2010) bestehen diesbezüglich erhebliche Unterschiede. Dennoch gibt es zwischen den benachbarten Tälern zahlreiche floristische Gemeinsamkeiten. Das Trisetetum flavescentis des Untersuchungsgebietes weist außerdem eine gute Übereinstimmung mit der Originalbeschreibung dieser Assoziation nach Rübel (1911) im Berninagebiet auf. Enge floristische Bezüge gibt es auch zu den Aufnahmen von Ellmauer (1994) aus Tirol und mit dem Trisetetum flavescentis, das Dierschke (1979) in der Umgebung von Galtür untersucht hat. Die Goldhaferwiesen weisen hinsichtlich ihrer Artenzusammensetzung Bezüge zum Trisetetum flavescentis auf, welches Brugger (2011) auf der Pidigalm im Gsieser Tal in Südtirol zwischen 1620 und $2060 \mathrm{~m}$ Meereshöhe untersucht hat.

\section{Diskussion}

Die Bewirtschaftungsintensität (Mahd, Düngung) kann eindeutig als wichtigster Faktor für die Ausbildung der Pflanzengesellschaften in Ober- und Untergurgl 
betrachtet werden. Meereshöhe und Neigung stehen mit dieser in direktem $\mathrm{Zu}$ sammenhang: abgelegene und steilere Flächen werden extensiver bewirtschaftet oder wurden gänzlich aufgelassen. Mit zunehmender Nutzungsintensität nimmt die Artendiversität immer mehr ab. Unter den Umweltparametern überlagerte die Exposition (ausgedrückt durch den Hitze-Index) den Einfluss der Bewirtschaftung. Der pH-Wert des Bodens spielte hingegen keine Rolle.

Das Trisetetum flavescentis wird deutlich intensiver bewirtschaftet als das SieversioNardetum strictae. Besonders gravierend wirkte sich die intensive Nutzung auf die Artenvielfalt aus: in Tallage betrug die mittlere Anzahl der Arten pro Aufnahmefläche nur noch 16. Diese Flächen sind vermutlich planiert worden und werden von Arten der Fettwiesen beherrscht. Durch die Einebnung gehen Strukturunterschiede im Mikrorelief verloren, welches vor allem im Berggebiet vielen verschiedenen Arten Wuchsmöglichkeiten bietet. Die intensive Düngung der Talmähwiesen wiederum wirkt sich ausgleichend auf die Bodenbeschaffenheit aus, wobei Unterschiede in den Standortfaktoren kompensiert werden (Marschall 1951). Die Düngung von Goldhaferwiesen fördert zudem wenige, angepasste krautige Arten (Dietl 1988).

Das Trisetetum flavescentis nardetosum vermittelt zwischen den Goldhaferwiesen und Bürstlingsrasen, ist jedoch durch die Mahd sowie durch die Artausstattung eindeutig den Goldhaferwiesen zuzuordnen. Im Unterschied zum Trisetetum flavescentis typicum werden die NardetumWiesen nur einmal im Jahr gemäht, auch die Düngung ist nicht mehr so intensiv. Die Artenzahl ist gegenüber der intensiver bewirtschafteten Subassoziation typicum deutlich erhöht, erreicht aber bei weitem nicht den Wert der Bürstlingsrasen. Die einschürigen Wiesen schließen sich unmittelbar an die Tallagen an (Nagl 2009). Die Mahd wird zunehmend durch Beweidung ersetzt. Die Nutzung als Weide ist für das Sieversio-Nardetum strictae charakteristisch und generell gelten Borstgrasrasen als die typischen Magerweiden in der subalpinen Stufe (Dietl 1998) zwischen 1800 und 2200 m Meereshöhe (Oberdorfer 1978, Lüth et al. 2011b). Im Gegensatz zu den gemähten Goldhaferwiesen weisen Weideflächen eine höhere Vielfalt an unterschiedlichen Strukturen im Mikrorelief und dadurch eine höhere Artenvielfalt auf (Dierschke \& Briemle 2002).

Die Bürstlingsrasen werden durch die Horste des Borstgrases bzw. Bürstlings (Nardus stricta) geprägt. Dieses Gras wurde vorwiegend sekundär durch die menschliche Tätigkeit gefördert (Reisigl $\&$ Keller 1994). Die Art war wahrscheinlich ursprünglich in länger schneebedeckten Lagen in Lawinen der hochmontanen und subalpinen Stufe verbreitet. Aufgrund der Anspruchslosigkeit gegenüber den Bodenverhältnissen und wegen der Resistenz gegenüber Viehtritt und Verbiss 
hat sich Nardus stricta auf den Almen ausgebreitet (Reisigl \& Keller 1994).

Die Studie in Obergurgl zeigte, dass sich die drei Subassoziationen des SieversioNardetum strictae vor allem durch die Bewirtschaftungsweise unterscheiden, wobei eine zunehmende Nutzungsintensität von der Subassoziation vaccinietosum über typicum bis hin zu trifolietosum pratensis festzustellen war. Unter den Umweltfaktoren spielte die Meereshöhe die wichtigste Rolle. Die zwergstrauchreichen, höher gelegenen Flächen wurden bereits großteils vor mehr als 10 Jahren aufgelassen. Der deutliche Rückgang der Zwergsträucher in den Subassoziationen typicum und trifolietosum pratensis hat damit zu tun, dass einige der Flächen halb- bzw. einschürig gemäht und mit Mist, aber auch mit Mineraldünger gedüngt werden (Abb. 8). Mahd und Düngung fördern anspruchsvollere Hemikryptophyten (Peppler-Lisbach \& Petersen 2001), während sich bei extensiver Beweidung Zwergsträucher ausbreiten können ( $\mathrm{Nagl}$ 2009). Für die Differenzierung des Sieversio-Nardetum strictae in die Subassoziationen spielen aber auch die Bodenverhältnisse eine Rolle. Die Subassoziation trifolietosum pratensis gedeiht vor allem an Standorten mit tiefgründigen, frischen Böden, welche eine gute Nährstoffver-

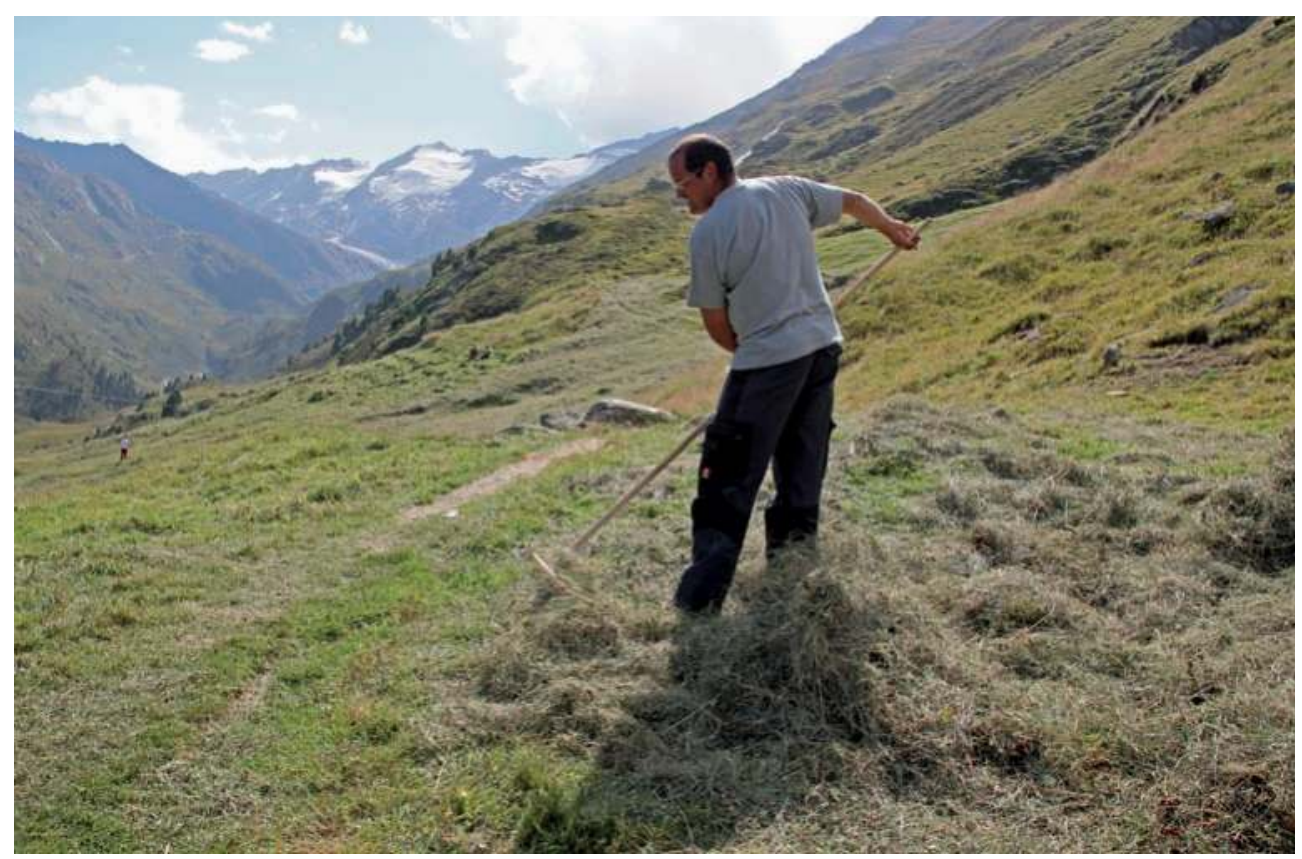

Abb. 8:

Einzelne der abgelegeneren Bergwiesen werden auch heute noch gedüngt und mit der Hand gemäht. Der Abtransport ins Tal erfolgt mit einer Materialseilbahn. (Foto: Brigitta Erschbamer) 
sorgung aufweisen. Die beiden anderen Subassoziationen sind hingegen auf flachgründigeren und trockeneren Böden mit mäßiger bis geringer Nährstoffversorgung ausgebildet (Nagl 2009).

Anders als bei den Goldhaferwiesen wurden bei den Bürstlingsrasen in Obergurgl schon zahlreiche Flächen wegen ihrer Abgelegenheit oder Steilheit aufgegeben. Gänzlich aufgelassene Bestände verbrachen allmählich, jedoch hängt diese Entwicklung mehr von den Standortverhältnissen wie Meereshöhe, Feuchtigkeit und $\mathrm{pH}$-Wert des Bodens ab als vom Alter der Brache. Die Subassoziation vaccinietosum weist den höchsten Anteil an alten Brachen auf. In höheren Lagen benötigen Zwergsträucher Jahrzehnte, um in aufgelassene Bestände einzuwandern (Tasser \& Tappeiner 2002). In zahlreichen Untersuchungen wurde festgestellt, dass der Anteil der Gräser, wie vor allem von Carex sempervirens und Molinia caerulea, deutlich zunimmt (z.B. Steinmair 1999, Tasser \& Tappeiner 2002, Mayer $\&$ Grabner 2004). In Obergurgl sind es jedoch vor allem die Zwergsträucher, die zur Dominanz gelangen. Auffallend war, dass selbst in den alten Brachen nur ganz vereinzelt Keimlinge oder Jungwuchs von Bäumen (Larix decidua, Pinus cembra) $\mathrm{zu}$ beobachten waren. Das könnte mit der südlichen Exposition der Flächen in Zusammenhang stehen. Trockenere Bedingungen können sich nachteilig auf die Wiederbewaldung von Brachflächen auswirken (Maag et al. 2001). Vor allem der hohe Anteil an Nardus stricta und an Zwergsträuchern dürfte sich verzögernd auf die Ansiedlung von Gehölzen auswirken. Von Gräsern dominierte Gesellschaften zeichnen sich durch ein dichtes Wurzelwerk aus, zudem erschwert die Streuansammlung ein Aufkommen von Baumkeimlingen (Maag et al. 2001).

Die Diversität des Sieversio-Nardetum strictae änderte sich im Untersuchungsgebiet kaum (35-40 Arten/Aufnahmefläche). Selbst in der Subassoziation vaccinietosum mit dem größten Anteil an alten Brachen gab es keinen Rückgang der Artenvielfalt. Demgegenüber wurde jedoch in anderen Studien vielfach eine Abnahme der Artenzahl bei der Brachlegung extensiver Bergwiesen und -weiden festgestellt (z.B. Maag et al. 2001, Tasser \& Tappeiner 2002, Niedrist et al. 2009). Allerdings herrscht in der Literatur keine generelle Einigkeit darüber.

\section{Literatur}

Aulitzky, H., Fromme, G., Schiechtl, H.M. \& Stern, R. (1961) Beschreibungen des Gurglertales. In: Ökologische Untersuchungen in der subalpinen Stufe zum Zwecke der Hochlagenaufforstung. Teil I. Mitteilungen der Forstlichen BundesVersuchsanstalt Mariabrunn 59: 33-52.

Austrheim, G., Gunilla, E., Olsson, A. \& Grøntvedt, E. (1999) Land-use impact 
on plant communities in semi-natural sub-alpine grasslands of Budalen, central Norway. Biological Conservation, 87: 369-379.

Bätzing, W. (1996) Landwirtschaft im Alpenraum - unverzichtbar, aber zukunftslos? Eine alpenweite Bilanz der aktuellen Probleme und der möglichen Lösungen. Blackwell, Berlin, 242 S.

Braun-Blanquet, J. (1964) Pflanzensoziologie. Grundzüge der Vegetationskunde. Springer Verlag, Wien, 865 S.

Brugger, B. (2011) Die Wiesenvegetation der Pidigalm (Gsieser Tal, Südtirol). Masterarbeit, Universität Innsbruck, 100 S.

Dietl, W. (1988) Standort und Verbreitung der Kräuter in unseren Dauerwiesen. Schweizerische Landwirtschaftliche Forschung. Recherche agron. en Suisse, 27/2: 117-125.

Dietl, W. (1998) Wichtige Pflanzenbestände und Pflanzenarten der Alpweiden. Agrarforschung 5 (6): 1-8.

Dierschke, H. \& Briemle, G. (2002) Kulturgrasland. Wiesen, Weiden und verwandte Staudenfluren. Ökosysteme Mitteleuropas aus geobotanischer Sicht. Ulmer Verlag. Stuttgart, 239 S.

Dierschke, H. (1979) Grünlandgesellschaften im oberen Patznauner Tal (Tirol/Österreich). Phytocoenologia, 6: 287-302.

Dierschke, H. (1994) Pflanzensoziologie. Grundlagen und Methoden. Verlag Eugen Ulmer, Stuttgart, 684 S.

Dufrêne, M. \& Legendre, P. (1997) Species assemblages and indicator species: the need for a flexible asymmetrical approach.
Ecological Monographs, 67/3: 345-366.

Ebus, A. (1986) Auswirkungen des Wintersports auf alpine Hänge - eine Schadenskartierung im Skigebiet Gurgler Tal/Ötztal, Tirol. Diplomarbeit, Geographisches Institut Rheinische FriedrichWilhelm-Universität, Bonn.

Ellenberg, H., Weber, H.E., Düll, R., Wirth, V., Werner, W. \& Paulißen, D. (1992) Zeigerwerte von Pflanzen in Mitteleuropa. Scripta Geobotanica 18, 2. Auflage.

Ellmauer, T. (1994) Die Syntaxonomie der Frischwiesen (Molinio-Arrhenatheretea p.p.) in Österreich. Tuexenia, 14: 151168.

Ellmauer, T. (1995) Nachweis und Variabilität einiger Wiesen- und Weidegesellschaften in Österreich. Verhandlungen der Zoologisch-Botanischen Gesellschaft in Österreich, 132: 13-60.

Ellmauer, T. \& Mucina, L. (1993) MolinioArrhenatheretea. In: Die Pflanzengesellschaften Österreichs. Teil I: Anthropogene Vegetation. Mucina, L., Grabherr, G. \& Ellmauer, T. (Hg.) Gustav Fischer. Jena, Stuttgart, New York: 297-401.

Fischer, M.A., Oswald, K. \& Adler, W. (2008) Exkursionsflora für Österreich, Liechtenstein und Südtirol. 3. Auflage. Biologiezentrum der Oberösterreichischen Landesmuseen, Linz, 1394 S.

Grabherr, G. (1987) Tourismusinduzierte Störungen, Belastbarkeit und Regenerationsfähigkeit der Vegetation in der alpinen Stufe. In: MaB-Projekt Obergurgl, Patzelt, G. (Hg.) Wagner Innsbruck, Band 10: 243-256. 
Grabherr, G. (1993) Caricetea curvulae. In: Grabherr, G. \& Mucina, L. (Hg.) Die Pflanzengesellschaften Österreichs. Teil II: Natürliche waldfreie Vegetation. Gustav Fischer, Jena, Stuttgart, New York: 343-372.

Grabner S. \& Heiselmayer P. (2002) Diversity of Mountain meadows in the inner alpine valley Virgental/Eastern Tyrol. Razprave IV. Razreda Sazu, XLIII-3: 167-184.

Heiselmayer, P. (1982) Die Pflanzengesellschaften des Tappenkars (Radstädter Tauern). Stapfia, 10: 161-202.

Lüdi, W. (1948) Die Pflanzengesellschaften der Schynige Platte bei Interlaken und ihre Beziehung zur Umwelt. Eine vergleichende ökologische Untersuchung. Veröffentlichungen des Geobotanischen Instituts Rübel, 23: 1-400.

Lüth, C., Tasser, E., Niedrist, G., Della Via, J. \& Tappeiner, U. (2011a) Plant communities of mountain grasslands in a broad cross-section of the Eastern Alps. Flora, 206: 433-443.

Lüth, C., Tasser, E., Niedrist, G., Della Via, J. \& Tappeiner, U. (2011b) Classification of the Sieversio montanae-Nardetum strictae in a cross-section of the Eastern Alps. Plant Ecology, 212: 105-126.

Maag, S., Nösberger, J. \& Lüscher, A. (2001) Mögliche Folgen einer Bewirtschaftungsaufgabe von Wiesen und Weiden im Berggebiet. Ergebnisse des Komponentenprojektes D, Polyprojekt PRIMALP. Graslandwissenschaften, ETH Zentrum, Zürich: 1-58.
Marschall, F. (1951) Beiträge zur Kenntnis der Goldhaferwiese (Trisetetum flavescentis) der Schweiz. Vegetatio, 3: 195-209.

Maurer, K., Weyand, A., Fischer, M. \& Stöcklin, J. (2006) Old cultural traditions, in addition to land-use and topography, are shaping plant diversity of grasslands in the Alps. Biological Conservation, 130: 438-446.

Mayer, R. \& Grabner, S. (2004) Die Vegetation der Bergmähder im Valsertal/Tirol. Tuexenia, 24: 227-246.

Mayer, R., Kaufmann, R., Vorhauser, K. \& Erschbamer, B. (2009) Effects of grazing exclusion on species composition in high-altitude grasslands of the Central Alps. Basic and Applied Ecology, 10: 447-455.

Mayer, R., Nagl, F., Unterluggauer, P. \& Erschbamer, B. (2010) Wirtschaftsgrünland zweier benachbarter Seitentäler im inneren Ötztal (Zentralalpen, Tirol/Österreich). Tuexenia, 30: 129-148.

McCune, B. \& Grace, J.B. (2002) Analysis of Ecological Communities. MjM Software Design, Gleneden Beach, Oregon, 300 S.

Nagl, F. (2009) Vegetationskundliche Untersuchungen von Wiesen und Weiden im Raum Obergurgl. Diplomarbeit, Universität Innsbruck, 129 S.

Niedrist, G., Tasser, E., Lüth, C., Della Via, J. \& Tappeiner, U. (2009) Plant diversity declines with recent land use changes in the European Alps. Plant Ecology, 202: 195-210

Oberdorfer, E. (1978) Süddeutsche Pflanzengesellschaften. Teil II: Sand- und Tro- 
ckenrasen, Heide- und Borstgras-Gesellschaften, alpine Magerrasen, Saum-Gesellschaften, Schlag- und HochstaudenFluren. Bd. 2, Gustav Fischer, Stuttgart, $355 \mathrm{~S}$.

Patzelt, G. (1996) Modellstudie Ötztal Landschaftsgeschichte im Hochgebirgsraum. Mitteilungen der Österreichischen Geographischen Gesellschaft, 138: 53-70.

Peppler-Lisbach, C. \& Petersen, J. (2001) Calluno-Ulicetea (G3). Teil 1: Nardetalia strictae. Borstgrasrasen. Synopsis der Pflanzengesellschaften Deutschlands, 8: $1-116$.

Reichelt, G. \& Wilmanns, O. (1973) Vegetationsgeographie. Westermann, Braunschweig, $210 \mathrm{~S}$.

Reisigl, H. \& Keller, R. (1994) Alpenpflanzen im Lebensraum. Alpine Rasen, Schuttund Felsvegetation. Gustav Fischer Verlag, Stuttgart, Jena, New York, 150 S.

Reisigl, H. \& Keller, R. (1999) Lebensraum Bergwald: Alpenpflanzen in Bergwald, Baumgrenze und Zwergstrauchheide, 2. Auflage. Spektrum Akademischer Verlag, Gustav Fischer, Heidelberg, Berlin, $158 \mathrm{~S}$.

Rübel, E. (1911) Pflanzengeographische Monographie des Berninagebietes. Botanisches Jahrbuch Leipzig, 47: 1-646.

Rudmann-Maurer, K., Weyand, A., Fischer, M. \& Stöcklin, J. (2008) The role of landuse and natural determinants for grassland vegetation composition in the Swiss Alps. Basic and Applied Ecology, 9/5: 494-503.
Steinmair, V. (1999) Die Vegetation von unterschiedlich genutzten Almflächen auf der Plätzwiese (Dolomiten, Südtirol). Diplomarbeit, Universität Innsbruck, $134 \mathrm{~S}$

Tasser, E. \& Tappeiner, U. (2002) Impact of land use changes on mountain vegetation. Applied Vegetation Science, 5: 173-184.

Tasser, E., Mader, M. \& Tappeiner, U. (2003) Effects of land use changes in alpine grasslands on the probability of landslides. Basic and Applied Ecology, 4/3: 271-280.

Tasser, E., Walde, J., Tappeiner, U., Teutsch, A. \& Noggler, W. (2007) Land-use changes and natural reforestation in the Eastern Central Alps. Agriculture, Ecosystems and Environment, 188: 115-129.

Tasser, E., Ruffini, F.V. \& Tappeiner, U. (2009) An integrative approach for analysing landscape dynamics in diverse cultivated and natural mountain areas. Landscape Ecology, 24: 611-628.

Ter Braak, C.J.F. \& Šmilauer, P. (1998) CANOCO Reference Manual and User's Guide to Canoco for Windows. Software for Canonical Community Ordination (version 4). Wageningen: Centre for Biometry, $352 \mathrm{~S}$.

Unterluggauer, P. (2003) Die Vegetation in Vent und Rofen (Ötztal Tirol). Diplomarbeit Universität Innsbruck, 166 S.

Wagner, H. (1965) Die Pflanzendecke der Kompardellalm in Tirol. Documents pour la Carte de la Végétation des Alpes, 3: 7-59. 
Verzeichnis der AutorInnen

Brigitta Erschbamer

Institut für Botanik

Universität Innsbruck

Sternwartestr. 15, 6020 Innsbruck

Brigitta.Erschbamer@uibk.ac.at

Roland Mayer

Institut für Botanik

Universität Innsbruck

Sternwartestr. 15, 6020 Innsbruck

Roland.Mayer@uibk.ac.at
Fabian Nagl

Institut für Botanik

Universität Innsbruck

Sternwartestraße 15, 6020 Innsbruck

Fabian.Nagl@uibk.ac.at 


\section{Kapitel 2 I Die Landschaft und ihre Namen - \\ Landwirtschaftliche Nutzung und Nutzungsände- rungen im Spiegel der Flurnamen von Obergurgl und Vent (Ötztal)}

Lorelies Ortner, Rüdiger Kaufmann, Yvonne Kathrein, Johanna Pidner

\section{Zusammenfassung}

Flurnamen wurden zu allen Zeiten von Hirten und Bauern zur Orientierung und zur Kommunikation benützt. Die reich strukturierte Hochgebirgslandschaft sowie jahrhundertelange Besitzteilungen haben in Obergurgl und in Vent eine große Vielfalt an Toponymen (Örtlichkeitsnamen) begünstigt.

In Interviews mit Einheimischen wurden 1255 Flurnamen erhoben. Zudem wurde das Erfahrungswissen über Landnutzung und Nutzungsänderung festgehalten. Die Fluren wurden GIS-kartiert und fotografisch dokumentiert.

Einige Flurnamen stammen bereits aus einer vorrömischen Sprachschicht oder haben romanische Wurzeln. Die Mehrzahl aber basiert auf dem Deutschen. Seit dem Hochmittelalter wird benannt, was für die Berglandwirtschaft auffällig und wichtig war. Die Flurnamen beziehen sich vor allem auf Geländeformen wie z. B. Rinne, ferner auf die Lage der Fluren (Äußere Wiese - Innere Wiese) und ihre Größe (Winkele). Wichtig war auch die Nennung der Besitzer (Jakoben Wald).
Die Methode der Flurnamenerhebung eignet sich hervorragend als Ausgangspunkt für Alltagsgeschichten über das bergbäuerliche Leben in Vergangenheit und Gegenwart. Die Untersuchung leistet somit nicht zuletzt auch einen Beitrag zur Erhaltung des kulturellen Erbes.

\section{Abstract}

At all times field names have been used by shepherds and farmers as an aid to orientation and as a code for communication. The richly structured alpine landscape as well as the century-old tradition of property partitioning have promoted a high diversity of toponyms in Obergurgl and Vent.

1,255 toponyms have been surveyed in interviews with locals. In addition, the knowledge about land use and land use change has been recorded. The named areas were mapped in a GIS and documented photographically. Some field names have their origin in a preRomanic language layer or have Romanic roots. The majority, however, is German- 
based. Since the High Middle Ages names have been given to conspicuous places or places important for mountain agriculture. Toponyms refer to terrain shape such as Rinne (trough), to the location of a given area (e.g. Äußere Wiese - Innere Wiese: inner meadow outer meadow) and their size (e.g. Winkele. little corner). The inclusion of the landowner in the name was also important: Jakoben Wald (Jacob's wood).

The investigation of toponyms is an excellent starting point to study everyday life as well as the habits of mountain farmers, both past and present. Thus, this research is a contribution to the preservation of the region's cultural heritage.

\section{Einleitung}

Bereits vor mehr als 9000 Jahren wanderten Hirten auf die hochgelegenen Bergwiesen oberhalb von Obergurgl und Vent, um ihre Schafe zu weiden (persönl. Mitteilung, Alexander Zanesco; siehe auch S. 75 ff.). Im Hochmittelalter entwickelten sich die Orte zu Dauersiedlungen: Urkundlich erwähnt wurden Vent und Gurgl erstmals 1241 bzw. 1250 (Stolz 1953: 21, Stolz 1939: 77, Stolz 1963: 193 , Meixner/Siegl 2010: 22; für die weitere Entwicklung siehe Scharr 2001: Karten 2, 3). Mit der landwirtschaftlichen Nutzung der Hochgebirgslagen nahm der Mensch den Raum in Besitz, und zwar sowohl real als auch mental: Hirten und Bauern mussten sich in verschiedenartigen Landschaften orientieren, bestimmte Stellen in der Landschaft mental aussondern und identifizieren und vor allem: sie mussten mit anderen über spezifische Orte reden. Dies geschah, indem diese Örtlichkeiten Namen bekamen, die sie unterscheidbar machten, die Wichtiges und Auffälliges ins Bewusstsein riefen. Flurnamen sind „Anhaltspunkte“ in der Landschaft, aber auch im Kopf, „weil man sie braucht“. Das wissen die Einheimischen auch heute noch:

INT-P (= Interviewpartner): Ebm, die åltn Leit hattn nou viel mehr [Nåmen] gewisst. [...] Då ischt schon viel in Vergessenheit geråtn, weil man's nimmr braucht. [...] Kemmen tuat des hauptsächlich, glab i, durch's Bewirtschåftn und durch die Viechr. Weil de haim an Anbåltspunkt gebraucht. [...] Wenn heit sågscht „Die Schåf sein im Goaßbergtal" [...] INT (= Interviewer): Långt des. INT-P: Jå, schaut er mitn Guggr von dr Hohen Mut äba und weard sie schon findn. Frier, wenn nou koan Guggr kåbt håsch, [...] håt er genau wissn gewellt, bei welchm Stoan de gråd steahn.

INT-P: ,Eben, die alten Leute hätten noch viel mehr [Namen] gewusst. [...] Da ist schon viel in Vergessenheit geraten, weil man es nicht mehr braucht. [...] Kommen tut das hauptsächlich, glaub' ich, durch das Bewirtschaften und durch das Vieh. Weil die[se Leute] haben einen Anhaltspunkt gebraucht. [...] Wenn du heute sagst: „Die Schafe sind 
im Gaißbergtal“ [...]` INT: ,Genügt das.‘ INT-P: ,Ja, [da] schaut er mit dem Fernglas von der Hohen Mut herunter und wird sie schon finden. Früher, als du noch kein Fernglas gehabt hast, $[\ldots]$ da hat er [= der Bauer] genau wissen wollen, bei welchem Stein die [= das Vieh] gerade stehen.

Wie hängen nun die heutigen Flurnamen und die Landschaft zusammen? Welche mit Flurnamen benannten Landschaftselemente sind auch heute noch für die lokale Bevölkerung bedeutsam und welche Flurnamen sind in Vergessenheit geraten, weil die Flur nicht mehr wichtig ist? Was sagen uns die Flurnamen über ökologische Verhältnisse in früherer Zeit, über das Leben der Menschen „an den Grenzen des Waldes und der menschlichen Siedlung“, über die Nutzung der Hochgebirgslagen und auch die Nutzungsänderungen im Laufe der Zeit? Diese Fragen soll das Projekt Namenökologie beantworten.

\section{Namenökologie - die Verbindung von Flurnamenforschung und Land- schaftsökologie}

Im Projekt Namenökologie untersuchen wir seit 2008 die Flurnamen von Obergurgl und Vent, ihre Beziehung zur Landschaft und ihre Bedeutung für die lokale Bevölkerung. Wir erforschen die Zusammenhänge zwischen den durch Flurna- men geschaffenen, charakterisierten und bewerteten mentalen Räumen und den ökologischen Realräumen, die spezifisch gekennzeichnet werden können z.B. nach geomorphologischen Formationstypen oder nach Nutzungstypen (Kaufmann \& Ortner 2011). Es wurden 15 Interviews mit meist älteren Personen aus Obergurgl geführt sowie drei Interviews mit Bewohnern der Ortschaft Vent/Rofen, wobei dort ein Interviewpartner schon vor dem Interview vier weitere Auskunftspersonen beigezogen hatte. Das Berufsspektrum reicht von Landwirt/in, Bergund Wanderführer, Hüttenwirt, Hotelier und Wetterwart bis hin zu Jäger und Schäfer. In den Interviews war für uns wichtig, nicht nur die Flurnamen zu erfassen ( $\rightarrow$ S. $42 \mathrm{ff}$.), sondern auch das Alltagswissen über die Landschaft, über landwirtschaftliche Praktiken früher und heute sowie über die Veränderungen in der Landnutzung durch die rasante Entwicklung des Tourismus in Obergurgl und Vent aufzunehmen und für künftige Generationen zu bewahren ( $\rightarrow$ S. 61 ff.). Im Rahmen der Interviews wurden die genannten Fluren auf Orthofotos eingezeichnet und später in GIS kartiert. Nach Möglichkeit wurden die Landschaftselemente auch durch Fotos dokumentiert.

Im Folgenden werden die Flurnamen der besseren Verständlichkeit wegen weitgehend nicht in ihrer dialektalen Form (z.B. Reatrinna), sondern in einer Standardschreibweise angeführt (Rotrinne), die an die Normen der offiziellen Daten- 
bank für geographische Ortsbezeichnungen (Toponymika) in Österreich, GEONAM, angelehnt ist. „Undurchsichtige“ Flurnamen werden erklärt, wobei dazu vor allem das Wörterbuch der Tiroler Mundarten von Josef Schatz, herausgegeben von Karl Finsterwalder, (Schatz 1955/1956 = WTM) sowie die Tiroler Ortsnamenkunde von Karl Finsterwalder (Finsterwalder $1990 \mathrm{a}=$ TONK) verwendet wurden.

Wir danken allen unseren Interviewpartnern und -partnerinnen herzlich für ihre Teilnahme an der Untersuchung; InterviewpartnerInnen in Obergurgl: Gamper Hans, Giacomelli Karl, Grüner Hansjörg ( $\dagger)$, Grüner Otto (†), Gufler Alfons, Gufler Sigl, Klotz Heinrich, Ribis $\operatorname{Burgl}(\dagger)$, Ribis Ronald, Santer Valentin, Scheiber Method, Scheiber Pius, Schöpf Annemarie, Strobl Meinrad, Zwischenbrugger Albert; in Vent: Klotz Franz, Pirpamer Luis $(\dagger)$, Scheiber Hubert $(\dagger)$.

Unser Dank geht auch an die Institutionen, die das Projekt „Namenökologie“ unterstützt haben: Alpine Forschungsstelle Obergurgl (Universität Innsbruck), Forschungsschwerpunkt „Alpiner Raum - Mensch und Umwelt" der Universität Innsbruck, Forschungszentrum „Berglandwirtschaft" der Universität Innsbruck, Universität Innsbruck (Förderbeiträge Aktion D. Swarovski), Land Tirol (Agrar und Ländlicher Raum), Land Tirol (Kultur).

\section{Flurnamen in Obergurgl und Vent}

Was wird benannt? Und was wird nicht (mehr) benannt?

Es wurden insgesamt 1255 verschiedene Flurnamen erhoben, und zwar 787 in Obergurgl und 468 in Vent (im Folgenden mit den Subskripten ${ }_{\text {OG }}$ und ${ }_{\mathrm{VE}}$ gekennzeichnet). Benannt werden Wiesen, Wälder, Hügel, Berge und Täler, ferner auffällige Landschaftselemente wie Steine und Felsen sowie Gewässer, Wege, Ställe und Heuhütten (vgl. diesen sehr weiten Begriff von Flurnamen auch bei Waser 2004: 350), z.B.

Oberer Anger $_{\mathrm{OG}}$, Jakobn Wald ${ }_{\mathrm{OG}}$, Schön$\boldsymbol{b i c h l}_{\mathrm{OG}}$ ('gut nutzbarer Hügel'; vgl. Finsterwalder 1990 b: 475, Schönbichler), Kubberg VE $_{\text {' }}$ Äußeres Tal ${ }_{\mathrm{OG}}$, Milchstein ${ }_{\mathrm{VE}}$, Dreckbachle ${ }_{\mathrm{OG}}$, Alter Weg ${ }_{\mathrm{VE}}$, Mittlerer Alter Stall ${ }_{\mathrm{OG}}$, Spitzigensteinpille $_{\mathrm{OG}}$ (Abb.1) (Pille < rom. ${ }^{*}$ pila; Schorta 1964: 242 f., < lateinisch [= lat.] ,pilum 'Pfosten', also etwa 'auf Pfosten gesteltte [sic!] Heuhütte"“; TONK: 798; dieldas Pille = 'Heustadel, -hütte auf dem offenen Felde'; WTM: 79; vgl. auch WBÖ 3: 171); '*' bezeichnet hier und im Folgenden eine nicht belegte Wortform.

Welche Flurnamen werden heute in Obergurgl und Vent verwendet? Benannt werden sehr kleine Landschaftselemente in den Siedlungen selbst und in ihrer unmittelbaren Umgebung. Fern 


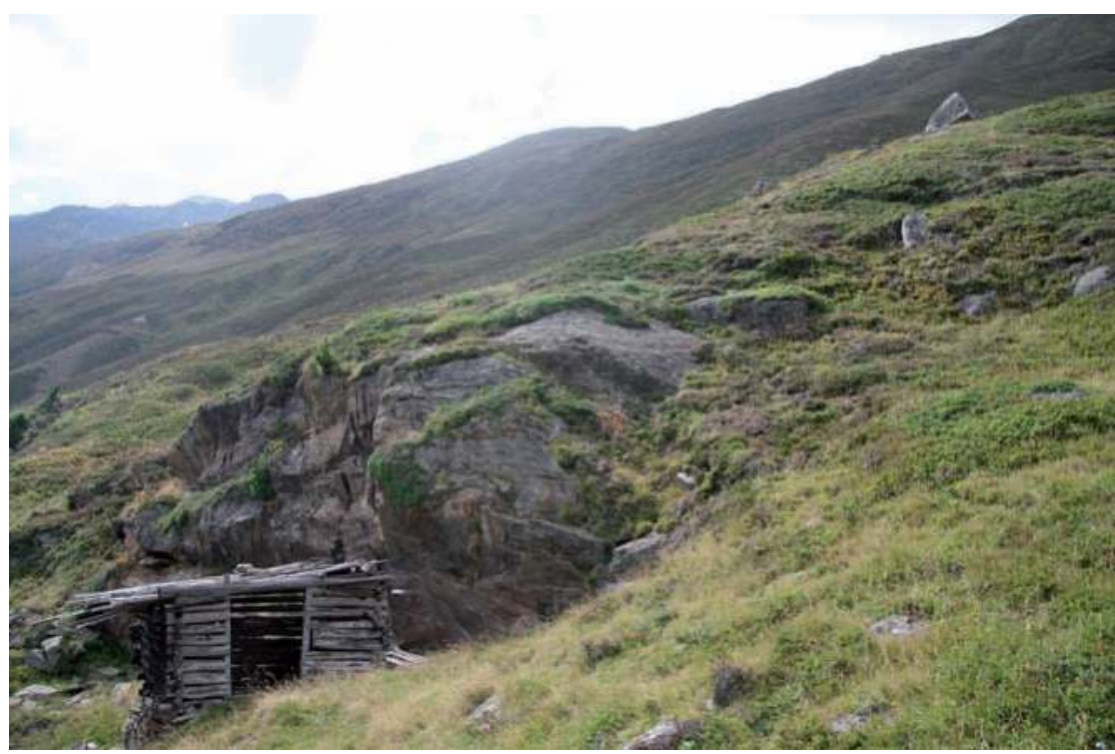

Abb. 1:

Pille ('Heuhütte') beim Spitzigen Stein ${ }_{\mathrm{OG}}$, einem markanten Landschaftspunkt (im Bild rechts oben). (Foto: R. Kaufmann)

der Siedlung sind durchwegs großflächige Namengebungen vorherrschend; die Grenzen der benannten Flächen werden unscharf und mit dem Abstand zu den Siedlungen gibt es auch zunehmend unbenannte Zwischenräume (Abb. 2).

Die Landschaftswahrnehmung durch die „Brille“ der Flurnamen ist funktional geprägt: Was entfernter ist, wird heute nicht mehr landwirtschaftlich genutzt, daher ist auch die detaillierte, möglicherweise flächendeckende Benennung früherer Zeiten in Vergessenheit geraten.

Benennungslücken sind auch den Interviewpartnern bewusst - und sie behelfen sich mit originellen Ersatzflurnamen, vgl. Namlos:
INT-P: Der Tuttn då, der kloane, des isch von mir aus der Namlos $_{\mathrm{OG}}$, zum Beispiel [...] Den håm $\mathrm{mr}$ allm den Namlos ghoaßn [...] Der Namlos isch namlos [...], weil er koan Nomn hàt. (Tuttn $\mathrm{m}$. < althochdeutsch tutta, $-o=$ 'Zitze, Brüste'; WTM: 669; wahrscheinlich: 'kleiner Berg, Hügel')

INT-P: ,Der Hügel da, der kleine, das ist von mir aus der Namlos $_{\mathrm{OG}}$, zum Beispiel [...] Den haben wir immer den Namlos genannt [...] Der Namlos ist namenlos [...], weil er keinen Namen hat.'

Der Bergname Namlos könnte allerdings auch elliptisch zum Rufnamen Amel gehören (Anreiter, Chapman \& Rampl 2009: 520). 


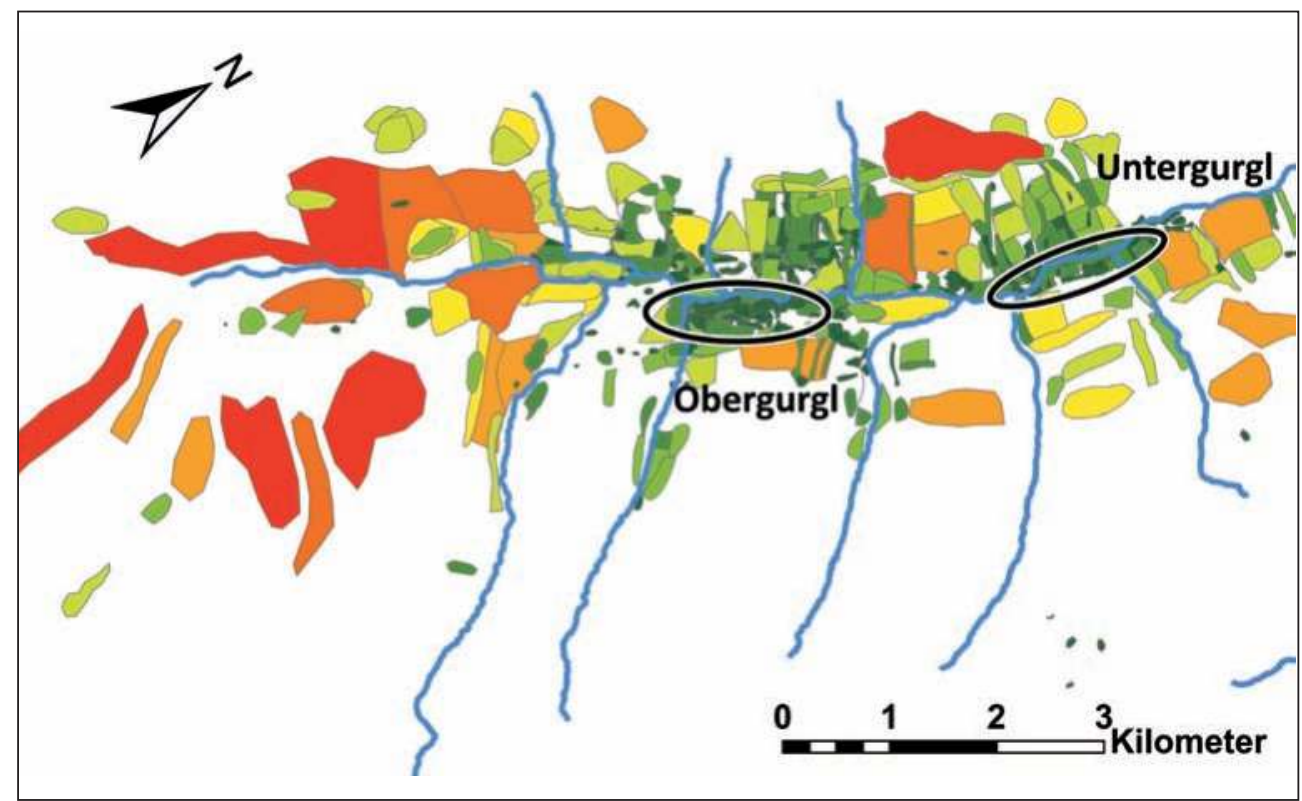

Abb. 2:

Räumliche Struktur der Fluren in Gurgl: Größe, Dichte und Flächendeckung der benannten Fluren. In den und um die Siedlungen sind die Fluren kleiner und daher ist die Benennungsdichte hoch (grün). Mit dem Abstand zur Siedlung werden die benannten Flächen größer, die Grenzen unschärfer und der Zusammenhang zwischen den Fluren löst sich allmählich auf (orange und rot).

Welche Gründe für einzelne Benennungslücken bestehen, muss noch genauer untersucht werden. Anzunehmen ist, dass vor allem das Aufgeben von landwirtschaftlichen Nutzflächen zugunsten der touristischen Nutzung für das Verschwinden von Namen verantwortlich ist. Aber auch Verschiebungen in den Besitzverhältnissen und schlechte Nutzbarkeit könnten Namen obsolet machen. Ein Beispiel: Abb. 3 zeigt eine unbenannte Fläche, die an die Fluren Poschiger Lärchlehne, Pixeler Lärchlehne und Friedls Lärchlehne anschließt (a). Ein weiteres unbenanntes Landschaftselement liegt zwischen den
Fluren Am Lärchen und Lehnerle (b). Beide Flächen könnten wegen schlechter Nutzbarkeit (z. B. unbequemer Zugang, Steilheit, schlechte Bodenqualität) landwirtschaftlich aufgegeben worden sein, die Besitzer bzw. Nutzer könnten daher nicht mehr bekannt sein. Die Folge könnte sein, dass die Benennung nicht mehr relevant und daher auch mental nicht mehr abrufbar ist.

Ein kurzer Blick in historische Quellen zeigt einen reichen Flurnamenschatz in früheren Zeiten. Das „Tagebuch der Kurazie und Gemeinde in Gurgl“" (Hofinger 1991) wurde von Kurat Adolf Trientl im 
Jahr 1858 angefangen. Die letzte Eintragung von Pfarrer Franz Danler erfolgte wahrscheinlich 1936. Hier einige Flurna- men, die in den Interviews nicht genannt wurden, aber im Pfarrtagebuch enthalten sind (Tab. 1).

Tab. 1:

Vermutlich untergegangene Gurgler Namen aus dem „Tagebuch der Kurazie und Gemeinde in Gurgl“ (mit Worterklärungen)

\begin{tabular}{|c|c|}
\hline Flurname & Kontext, Quelle und Worterklärung \\
\hline Bühlsbödele & (Hofinger 1991: 19) \\
\hline Gurgler Lacke & $\begin{array}{l}\text { Man erzählt [...], daß man schon vor } 140 \text { Jahren ungefähr auf dem steinernen } \\
\text { Tisch Messe gelesen und dahin mit dem Kreuze gegangen, um das Anwachsen } \\
\text { des großen Ferners und Verherungen [sic!] der Gurgler Lacke abzubitten. (Ho- } \\
\text { finger 1991: } 5 \text { f.) [Ferner = 'Gletscher'] }\end{array}$ \\
\hline Jochmut & (Hofinger 1991: 66) [Mut = 'gerundete[r] Rücken'; TONK: 798] \\
\hline Mahdstublebene & Mit der eingetauschten Wiese - Mahdstublebene - [...] (Hofinger 1991: 145) \\
\hline Martles Bühel & (Hofinger 1991: 56) ['Martins Hügel', vgl. WTM: 416] \\
\hline Nesselrain & Nesselrain unter Königsrain (Hofinger 1991: 32) \\
\hline Puitweg & $\begin{array}{l}\text { Am 16.II.[1935] [...] ging eine Lawine in großer Breite und Mächtigkeit ge- } \\
\text { gen [den Ortsteil] Pirchitt nieder. [...] Das Hotel Gurgl würde kaum so tapfer } \\
\text { standgehalten haben, wenn nicht die gewaltige Lawinenmauer oberhalb des } \\
\text { Hotels den Haupptstoß [sic!] aufgefangen hätte. [...] Wehe Pirchitt, wenn das } \\
\text { Hotel „Gurgl“ nicht gewesen wäre. Der Puitweg war tief unter Lawinen be- } \\
\text { graben; von der zerstörten Walke sah man gar nichts mehr. (Hofinger 1991: } \\
\text { 156) [Puite < mittelhochdeutsch (= mhd.) biunte 'eingehegtes Grundstück' } \\
\text { (vgl. Haslinger 2009: 236; ebenso WBÖ 2: 1175)], [Walke = 'Werkstatt } \\
\text { zum Walken'; WTM: 685] }\end{array}$ \\
\hline Rote Ebene & rote Ebene [beim Rotmoosjoch] (Hofinger 1991: 121) \\
\hline Wendlsgarten & $\begin{array}{l}\text { (Hofinger 1991: 149) ['Garten bzw. Weideplatz des Wendelin'; mundart- } \\
\text { lich Gårtn; WTM: 206] }\end{array}$ \\
\hline
\end{tabular}




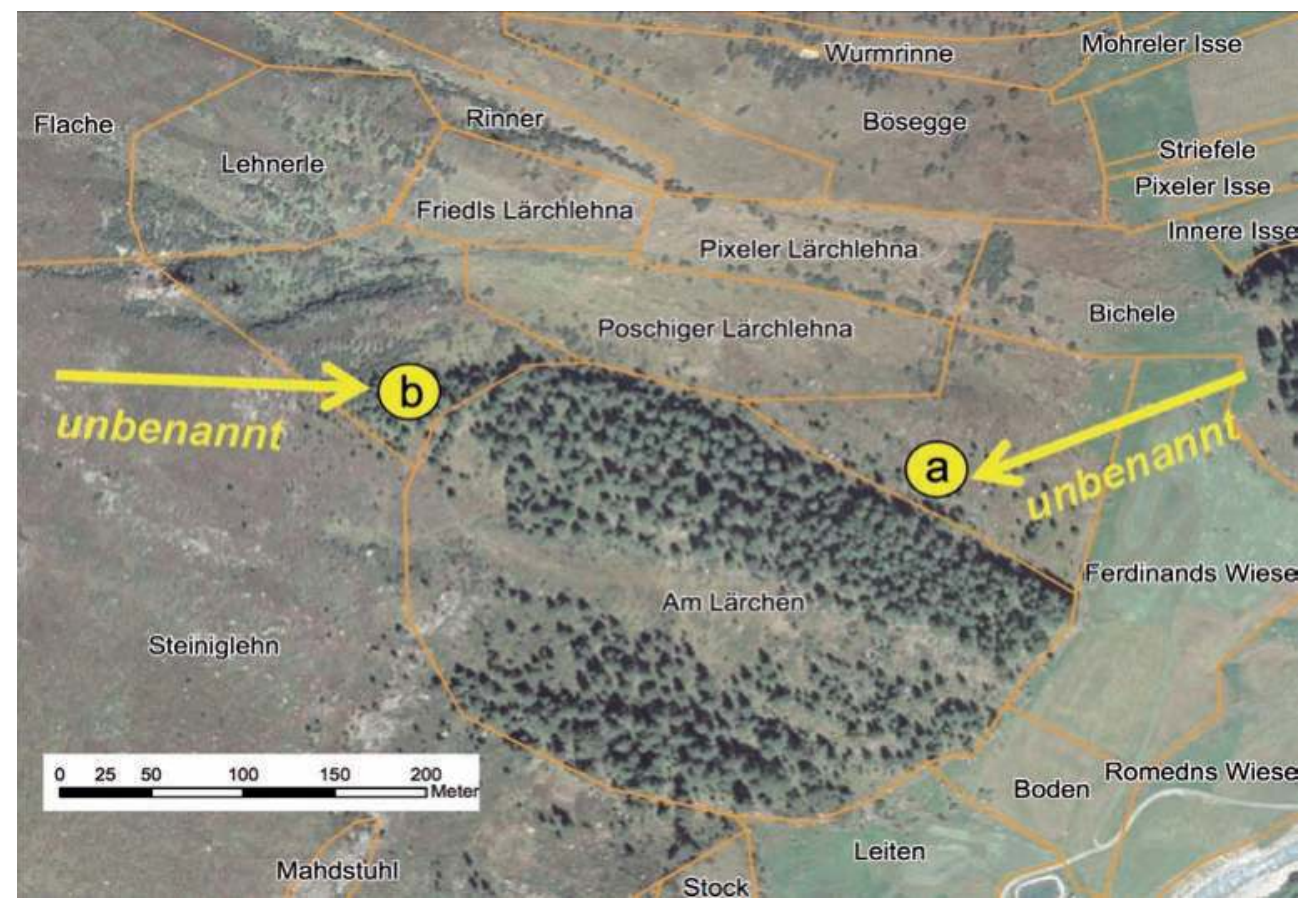

Abb. 3 a, b:

Unbenannte Fluren. Der Namenverlust könnte u.a. erklärbar sein durch schlechte Nutzbarkeit und Veränderung in den Besitzverhältnissen. (Orthofoto: Bundesamt für Eich- und Vermessungswesen = BEV; Befliegung 2003)

Das Tagebuch der Kurazie berichtet auch von einem Umbenennungsversuch, der anlässlich der weltweit Aufsehen erregenden Notlandung des Stratosphärenfliegers Auguste Piccard am 27. Mai 1931 auf dem Gurgler Ferner (Abb. 4) erfolgen sollte:

Piccardberg, Piccard-Höhe: Am 9. VI. 31 schrieb Graf Vojkiffy aus Oberstorf im Allgäu, daß der "Berg", auf dem Piccard [mit seinem Ballon] landete, nunmehr Piccardberg genannt werden solle. Ich antwortete ihm im $\mathrm{Na}$ men der Gemeinde zurück, daß wir dem Gro-
Ben Gugler Ferner [sic!] seinen alten Namen belassen, wohl aber die Landungsstelle PiccardHöhe nennen wollen. (Hofinger 1991: 138) $[$ Ferner $=$ 'Gletscher']

Der Flurname Piccardberg wurde von der Gemeinde abgelehnt - aber auch PiccardHöhe hat sich nicht bis heute erhalten.

Geht man noch weiter zurück in die Geschichte, zeigt sich ein sehr deutliches Bild des Namenverlustes. In den Jahren 1776/77 werden Fluren in Gurgl im Steuerkataster aufgenommen. 


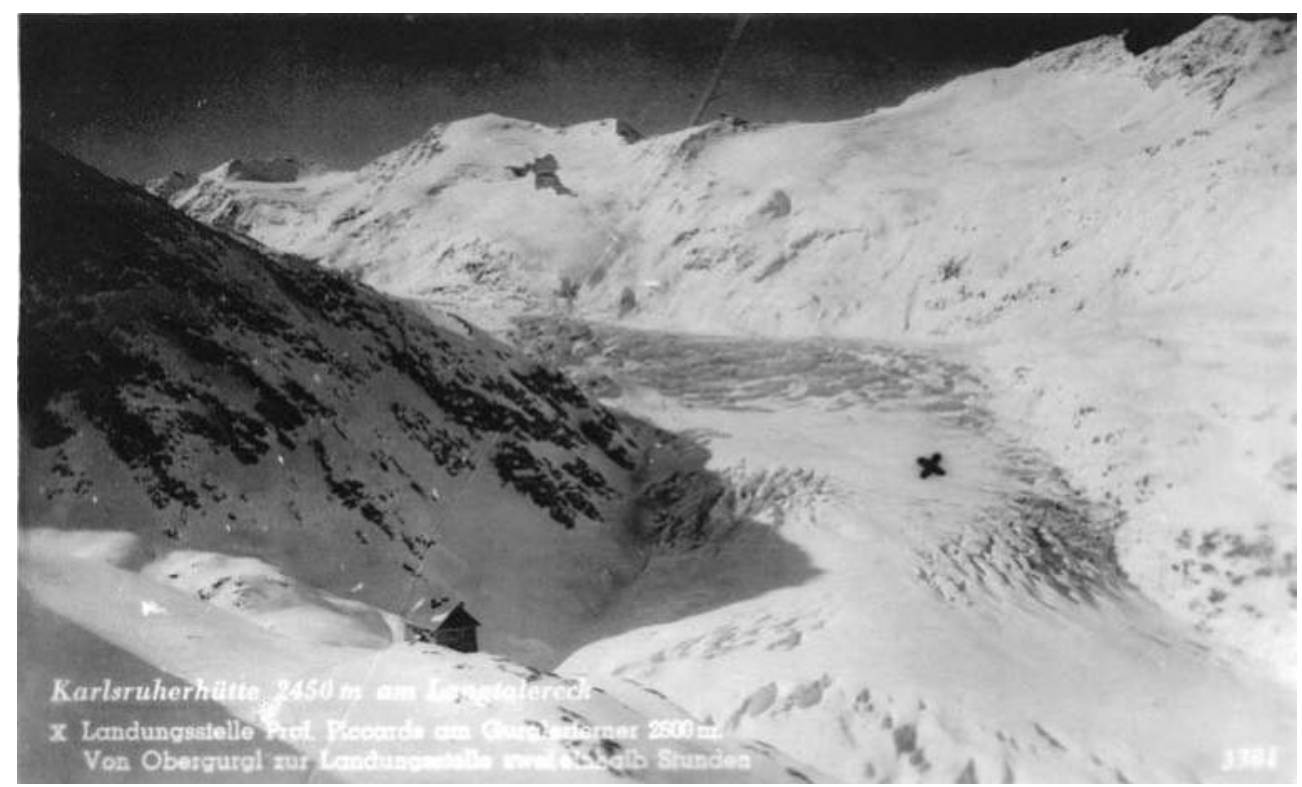

Abb. 4:

Stelle auf dem Gurgler Ferner, an der der Stratosphärenflieger Auguste Piccard am 27. Mai 1931 notgelandet ist

„Der Aufnahmezeitraum [1776/77] ist nicht exakt belegt, sondern aus dem Inhalt erschlossen. Die Katasteraufnahme in der Gemeinde Umhausen erfolgte im Jahre 1776." (Vorbemerkungen des Archivars, der den Kataster bearbeitet hat).

Der Theresianische Steuerkataster des Gerichtes Petersberg (Kat. 35/18-III, Tiroler Landesarchiv) verzeichnet zahlreiche Namen von Gurgl, die in den Interviews nicht genannt wurden und daher möglicherweise untergegangen sind (Tab. 2). Flurnamen und Flurnamenbestandteile, die heute nicht mehr in Verwendung sind, sind z.B. Lasse ('Spalte in großen Steinen'; WTM: 374) und Strange ('schmaler Streifen Feldes; einzelnes, zwischen zwei Furchen liegendes Ackerbeet'; Schmeller 1877, II: 816). Obwohl einzelne Flurnamen(bestandteile) verloren gegangen sind, sind die Prinzipien, nach denen die Wörter gebildet sind, dennoch weitgehend identisch mit denen, die den aktuellen Flurnamen zugrunde liegen (S. 51 ff.): Im Kataster kommen vor allem Zusammensetzungen wie Flachegge und Mehrwortlexeme wie Reiche Bachlen vor, sehr viel seltener Einzelwörter. 
Tab. 2:

Vermutlich untergegangene Gurgler Namen aus dem Theresianischen Steuerkataster des Gerichtes Petersberg aus den Jahren 1776/77 (Kat. 35/18-III; mit Worterklärungen)

\begin{tabular}{|c|c|}
\hline Flurname & Originalschreibweise, Katasterblatt und Worterklärung \\
\hline Alte Taja & $\begin{array}{l}\text { Auf der alten Theyen in d(en) Langen Wald [...] (fol. 223v); Taie < rom. } \\
{ }^{*} \text { teja }=\text { 'Sennhütte in den Alpen' (WTM: 626; ebenso WBÖ 4: } 1348 \mathrm{ff.} \text { ) } \\
<\text { lat. *tegia < * attegia < kelt. *ad-tegjâ (Anreiter, Haslinger 2005: } 38 \text { f.; } \\
\text { Haslinger 2009: 19) }\end{array}$ \\
\hline Flachegge & Am Fläch Egg [...] zu Ma(e)hen (fol. 187v) \\
\hline Grabengandlen & $\begin{array}{l}\text { Jn graben gandlen (fol. 227v); Gande < rom./vorröm. *gandâ = 'Schutt- } \\
\text { masse', bedeutet heute 'Hang mit Steingeröll' (WTM: 203) }\end{array}$ \\
\hline Obere Lasse & $\begin{array}{l}\text { auf den obern Läsße (fol. 230v); Lasse = 'Spalte in großen Steinen' (WTM: } \\
\text { 374) }\end{array}$ \\
\hline Pultstadl & $\begin{array}{l}\text { Bey den Pult(en) Stadl (fol. 225v); Pult: Hofname < Hl. Hippolytus (Fin- } \\
\text { sterwalder } 1990 \text { b: 223) }\end{array}$ \\
\hline Reiche Bachlen & Jnner den Reichen Pächlen (fol. 227v) \\
\hline Rinnenbachlen & Bey den Rinn(en) Pächlen (fol. 238v) \\
\hline Strange & $\begin{array}{l}\text { auf der Strangen (fol. 184v); 'schmaler Streifen Feldes; einzelnes, zwischen } \\
\text { zwei Furchen liegendes Ackerbeet' (Schmeller 1877, II: 816) }\end{array}$ \\
\hline Weihermoos & Weyer Mooß (fol. 230v) \\
\hline
\end{tabular}

Die Herkunft der Flurnamen: Namenschichten

Flurnamen werden zwar heute verwendet - aber wann sind sie entstanden? Die allerwenigsten Flurnamen in Obergurgl und Vent sind Namen der Gegenwart, wie z.B. die scherzhafte Bildung Schwiegermutterschlucht $_{\mathrm{OG}}$ oder der durch den Wintertourismus erklärbare Name Beim Starthüttle $_{\mathrm{VE}}$ (hier beginnen die Gäste- rennen). Aber ebenso ist festzuhalten, dass nur wenige Namen bzw. Flurnamenbestandteile wirklich alt sind, d.h. vordeutsche Wurzeln haben (mehr als 1200 Jahre alt). Alpenromanische (= rom.) oder gar noch ältere, vorrömische Wurzeln sind im Namenschatz beider Orte sehr selten (in Vent noch etwas häufiger als in Obergurgl). Die vordeutschen Namen(bestandteile) haben meist mit der Beweidung zu tun: es werden vor allem 
Tab. 3:

Romanische oder vorrömische Namen(bestandteile) bzw. eingedeutschte Namen(bestandteile) mit romanischer oder vorrömischer Wurzel (im Folgenden vereinfacht als „Vordeutsches in Flurnamen“ bezeichnet; in Klammern die deutschen Bestandteile des Flurnamens)

\begin{tabular}{|c|c|}
\hline $\begin{array}{l}\text { Vordeutsches in } \\
\text { Flurnamen }\end{array}$ & Herkunft und Bedeutung \\
\hline${\text { Verwall }(t a l)_{\mathrm{OG}}}$ & < rom. *val bella = 'schönes Tal' (TONK: 797; Anreiter 1997: 132) \\
\hline Timmels(joch) $_{\mathrm{OG}}$ & $\begin{array}{l}\text { < rom. }{ }^{*} \text { tumulu< lat. tumulus (Abl. loci }{ }^{*} \text { tumulis) }=\text { 'Hügel' (TONK: } \\
\text { 781; Anreiter 1997: 25) }\end{array}$ \\
\hline $\begin{array}{l}(\text { Hohe }) M u t_{\mathrm{OG}} \\
\text { Mut(boden) } \\
\mathrm{VE}\end{array}$ & $\begin{array}{l}\text { < rom. * muttâ 'gestutztes, abgerundetes Objekt' (Anreiter, Haslinger } \\
\text { 2005: } 33 \text { f.; Haslinger 2009: 203); heute: 'rundliche Erhebung, run- } \\
\text { der, stumpfer Berggipfel' (WTM: 440) }\end{array}$ \\
\hline $\begin{array}{l}(\text { Klein) alble } \\
\text { Alpe }_{\mathrm{VE}}\end{array}$ & $\begin{array}{l}\text { < vorindogermanisch *alp- 'Berg, Bergweide', mhd. albe (Haslinger } \\
\text { 2009: 17) }\end{array}$ \\
\hline $\begin{array}{l}\left(\text { Ochsen } \text { traje }_{\mathrm{OG}}\right. \\
\text { Traje }_{\mathrm{VE}}\end{array}$ & $\begin{array}{l}\text { < kelt. }{ }^{*} \text { tragju } /{ }^{*} \text { trogju (Anreiter, Haslinger 2005: 39), heute mund- } \\
\text { artlich Traje 'Weg für den Viehtrieb' (WTM: 650); die Ötztaler } \\
\text { Form ist umgelautet und unterscheidet sich damit von den nicht- } \\
\text { umgelauteten Trui-/Troi-Formen (WBÖ 5: 562) (Abb. 5) }\end{array}$ \\
\hline $\begin{array}{l}\text { Ramol(weg) } \\
\operatorname{Ramol}(\operatorname{alm})_{\mathrm{VE}}\end{array}$ & $\begin{array}{l}<\text { rom. }{ }^{*} \text { ru mal }\left(<{ }^{*} \text { riu malu }\right)=\text { 'übler Bach' (TONK: 792; Anreiter } \\
\text { 1997: 25) }\end{array}$ \\
\hline $\begin{array}{l}\text { (Wilde) Gande } \\
(\text { Große) Gande } \\
\text { (VE }\end{array}$ & $\begin{array}{l}<\text { rom./vorröm. * gandâa = 'Schuttmasse', bedeutet heute 'Hang mit } \\
\text { Steingeröll' (WTM: 203) }\end{array}$ \\
\hline $\begin{array}{l}\text { Gamples }(\text { kogel) } \\
\text { (Innerer) Gampen } \\
\text { (IE }\end{array}$ & $\begin{array}{l}\text { < rom. *campu< lat. campus = 'ebener freier Platz bei der Alphütte' } \\
\text { (WTM: 202; Haslinger 2009: 79 f.) }\end{array}$ \\
\hline Stablein $_{\mathrm{VE}}$ & $\begin{array}{l}\text { < rom. *stabulînu = 'kleiner Stall' (Finsterwalder 1975: 9; Anreiter } \\
\text { 1997: 25) }\end{array}$ \\
\hline $\left.\operatorname{Plattai}_{(\text {berg }}\right)_{\mathrm{VE}}$ & $\begin{array}{l}\text { < rom. *plattêdu = 'Plattach' (vgl. Finsterwalder 1975: 9); Plattach = } \\
\text { 'Gebiet mit Steinplatten' (WTM: 86) }\end{array}$ \\
\hline 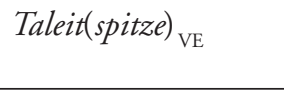 & $\begin{array}{l}<\text { rom. }{ }^{*} \text { taedulêdu = 'Ort mit Föhren, Kienholzbestand' (vgl. Finster- } \\
\text { walder 1975: 9; Anreiter 1997: 25) }\end{array}$ \\
\hline $\operatorname{Glaseir}_{(b a c h)_{\mathrm{VE}}}$ & $\begin{array}{l}<\text { rom. }{ }^{*} \text { clasüra, closüra < lat. }{ }^{*} \text { clausûra }=\text { 'Einfang, eingezäuntes } \\
\text { Grundstück' (vgl. TONK: 792, 797; Anreiter 1997: 25) }\end{array}$ \\
\hline Latsch $_{\mathrm{VE}}$ & $\begin{array}{l}\text { < rom. * vallacia = 'Hochtal'; } 1448 \text { Flatschbach (TONK: 796; Anrei- } \\
\text { ter 1997: 25) }\end{array}$ \\
\hline Taufen $_{\mathrm{VE}}$ & $\begin{array}{l}\text { < rom. *tovu < lat. tubus = 'Gleitbahn, -rinne für Stämme' (WTM: } \\
\text { 632; TONK: 798; WBÖ 4: 1077) }\end{array}$ \\
\hline Vent $t_{\mathrm{VE}}$ & < vorröm. *uend'b- 'Ort, Platz' (Anreiter 1997: 25) \\
\hline Rofen $_{\mathrm{VE}}$ & < vorröm. * rovinâ = 'Muhre' (TONK: 797; Anreiter 1997: 25) \\
\hline
\end{tabular}


hochgelegene Weideplätze, Gefahrenpotentiale bzw. für die Beweidung ungeeignete Plätze benannt (Tab. 3).

Die meisten der in Tab. 3 genannten Wörter sind Einzelwörter. Zwei Namenbestandteile aber, die ebenfalls auf vordeutsche Wörter zurückgehen, werden sehr häufig für reihenhafte Namenbildung genutzt: das sind die Flurnamen Kofel $_{\mathrm{OG}, \mathrm{VE}}$ (< lat. *cubulum = 'Höhle'; heute: 'großer Stein, Felsblock, Felswand'; WTM 347) und $K_{\text {ogel }}$ OG, VE $_{1}$ (< lat. cucullus = 'Kapuze'; heute: 'Bergkuppe, Berg, Bergspitze'; vgl. WTM 347; Rampl 2011: 136 f.), z.B.
Gampers Steinkofel ${ }_{\mathrm{OG}}$, Auenkofel ${ }_{\mathrm{OG}}$, Wasserkofel $_{\mathrm{VE}}$, Roter Kofel $\mathrm{VE}_{\mathrm{VE}}$; Plattenkogel ${ }_{\mathrm{OG}}$, Wurmkogel $_{\mathrm{OG}}$, Vorderer Spiegelkogel Die wenigen Flurnamen bzw. Flurnamenbestandteile, die aus vordeutschen Sprachschichten stammen, weisen darauf hin, dass die Weidegebiete um und in Obergurgl und Vent schon seit Jahrtausenden genutzt worden sind. Die meisten Flurnamen sind aber im Hochmittelalter und danach entstanden. Sie geben Hinweise auf die landschaftlichen und die landwirtschaftlichen Verhältnisse in der Zeit der Entstehung der dauerbesiedelten Schwaighöfe und später.

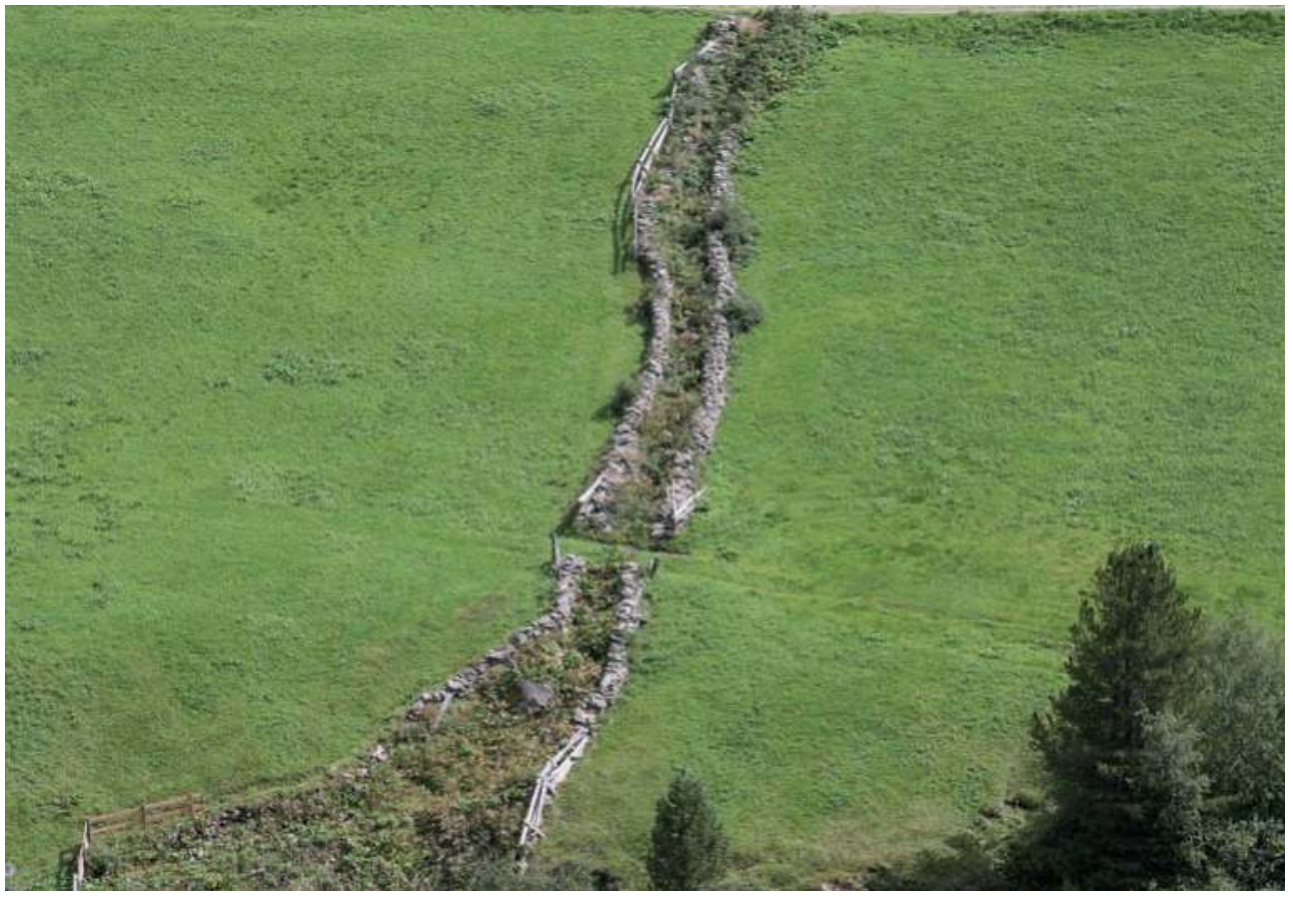

Abb. 5:

Hohlweg für den Viehtrieb: mundartlich Traje, ein Wort mit keltischen Wurzeln; heute wird dieser Viehweg in Obergurgl mit dem deutschen Flurnamen die Gasse benannt. (Foto: R. Kaufmann) 
"Schwaige' bedeutet die Haltung von Milchvieh und 'Schwaighöfe' waren Berghöfe, die zumeist von Grundherren mit einem gewissen Viehstande - etwa zu sechs Kühen und hundert Schafen - an Bauern zu Erbrecht und zur dauernden Bewirtschaftung gegen die jährliche Abgabe von 200 bis 300 kleinen Käsen verliehen waren. Da die Höfe oft knapp an oder ober [sic!] der Grenze des Getreidebaues liegen, erhielten die Bauern öfters von den Grundherren jährlich eine gewisse Menge Getreide zu ihrem Eigenbedarf zugewiesen. Die Höfe wurden vielfach auf Geländen, die bisher als Almwiesen genutzt waren, angelegt.“ (Stolz 1939: 75; vgl. auch Stolz 1930 und 1932)

Der größte Teil der Flurnamen ist also deutsch und wird heute im Dialekt des inneren Ötztals ausgesprochen: $d r$ Neadrboudn ${ }_{\mathrm{OG}}$ (Norder- 'schattenseitig'; WTM: 454), di Må:drgrüabe ${ }_{\mathrm{VE}}$ (Mähdergrube).

\section{Die Bildung der Flurnamen}

Flurnamen werden nicht aus dem Nichts heraus gebildet. Vielmehr werden vorhandene Wörter der Standardsprache für die Namengebung verwendet. Ein Beispiel: Im Innerötztaler Dialekt heißen Lawinen Lehnen und Lawinenstriche nennt man Lehner (WTM: 386). Aus vielen solchen Lehne(r)n wird eine(r) ausgesondert und erhält den individuellen Namen die Lehne bzw. der Lehner. Diese einfachen Flurnamen können dann weiterverwendet werden, um spezifische andere Landschaftselemente zu benennen, vgl. die dazugehörigen komplexeren Flurnamen in Obergurgl und Vent (Abb. 6).

Ähnliche Leitwörter wie Lehne bzw. Lehner gibt es viele. In Obergurgl werden die 787 erhobenen Flurnamen mit 194 verschiedenen Grundwörtern gebildet (also entsteht im Durchschnitt nur jeder 4. Name aus einer neuen Basis). Die Bewoh-

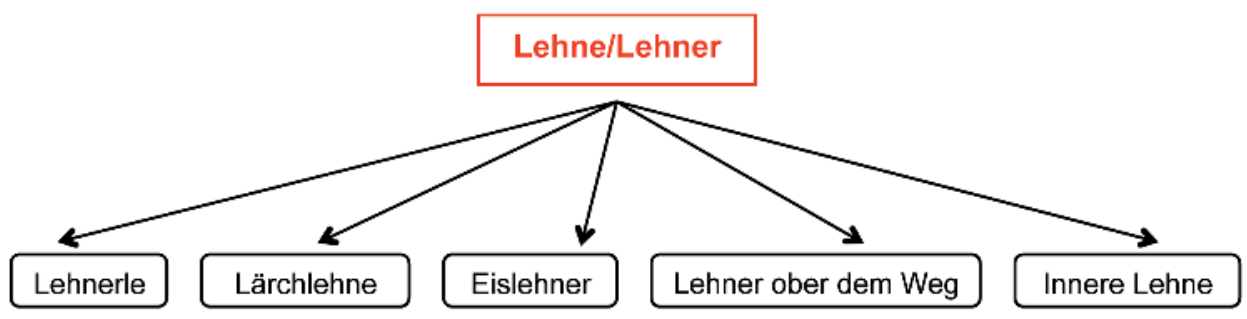

Abb. 6:

Vom einfachen zum komplexen Flurnamen: Ausgehend von den Basiswörtern die Lehne bzw. der Lehner entstehen Ableitungen, die aus einem Wort und einer Nachsilbe bestehen (Lehnerle ${ }_{\mathrm{OG}}$ ), ferner Zusammensetzungen aus zwei Wörtern (Lärchlehne ${ }_{\mathrm{OG}}$, Eislehne ${ }_{\mathrm{VE}}$ ) sowie Mehrwortfügungen (Lehner ober dem Weg ${ }_{\mathrm{VE}}$ ' Innere Lehne $\left.{ }_{\mathrm{OG}}\right)$. 
L. Ortner, R. Kaufmann, Y. Kathrein, J. Pidner

Tab. 4:

Die 15 häufigsten Namenbestandteile (Leitwörter) in den Flurnamen von Obergurgl und Vent (absteigende Reihenfolge): weitgehend gleichartige Verteilung der genutzten Wörter (vgl. Farbmarkierung)

\begin{tabular}{|c|c|c|c|c|c|}
\hline \multicolumn{3}{|c|}{ Obergurgl (787 erhobene Flurnamen) } & \multicolumn{3}{|c|}{ Vent (468 erhobene Flurnamen) } \\
\hline Pille & $\begin{array}{l}\text { 'Heustadel, -hütte auf dem } \\
\text { offenen Felde' (WTM: 79) }\end{array}$ & 34 & Egge & $\begin{array}{l}\text { '[...] vorstehendes Ende im } \\
\text { Gelände' (WTM: 141) }\end{array}$ & 22 \\
\hline Egge & $\begin{array}{l}\text { '[...] vorstehendes Ende im } \\
\text { Gelände' (WTM: 141) }\end{array}$ & 28 & Bach & & 18 \\
\hline Bach & & 27 & Stein & & 18 \\
\hline Ebene & & 18 & Köfele & 'kleiner Kofel' & 17 \\
\hline Lehne & 'Lawine’ (WTM: 386) & 17 & Kofel & $\begin{array}{l}\text { 'großer Stein, Felsblock, Fels- } \\
\text { wand' (WTM: } 347 \text { ) }\end{array}$ & 16 \\
\hline Barst & $\begin{array}{l}\text { 'Wiese mit Borstgras' (Nar- } \\
\text { dus stricta: schlechtes Gras) }\end{array}$ & 17 & Bachle & 'kleiner Bach' & 10 \\
\hline Infang & $\begin{array}{l}\text { 'Einfang, umzäuntes Stück } \\
\text { im Feld, Wald; Viehhag' } \\
\text { (WTM: 311) }\end{array}$ & 17 & Bichl & 'Hügel' & 10 \\
\hline Rinne & & 16 & Berg & & 9 \\
\hline Stein & & 16 & Bödele & 'kleine Bodenfläche' & 9 \\
\hline Tal & & 16 & Boden & $\begin{array}{l}\text { 'Boden, Bodenfläche' } \\
\text { (WTM: 94) }\end{array}$ & 9 \\
\hline Kar & $\begin{array}{l}\text { 'Im Hochgebirge Mulde, } \\
\text { Kessel als Weidegebiet' } \\
\text { (WTM: 324) }\end{array}$ & 15 & Ebenle & 'kleine Ebene' & 9 \\
\hline Köfele & 'kleiner Kofel' & 15 & Pille & $\begin{array}{l}\text { 'Heustadel, -hütte auf dem } \\
\text { offenen Felde' (WTM: 79) }\end{array}$ & 9 \\
\hline Kofel & $\begin{array}{l}\text { 'großer Stein, Felsblock, Fels- } \\
\text { wand' (WTM: 347) }\end{array}$ & 14 & Leger & $\begin{array}{l}\text { 'Lager, Lagerplatz für das } \\
\text { Vieh auf der Alpe, Weide' } \\
\text { (WTM: 381) }\end{array}$ & 8 \\
\hline Kogel & $\begin{array}{l}\text { 'Bergkuppe, Berg, Bergspit- } \\
\text { ze' (WTM: 347) }\end{array}$ & 14 & Rinne & & 8 \\
\hline$A u$ & & 12 & Feld & & 7 \\
\hline
\end{tabular}


ner von Vent verwenden 132 Grundwörter für die Bildung von 468 Flurnamen $(\varnothing=3,55)$. Tab. 4 zeigt die Hitparade der 15 häufigsten Namenleitwörter in Obergurgl und Vent. Es besteht kaum ein Unterschied zwischen den beiden Orten: Vorherrschend sind Naturnamen. Kulturnamen wie Pille, Infang oder Leger sind seltener reihenbildend.

Wie diese Leitwörter am Aufbau von „Namenfeldern“ (Fleischer 1962) beteiligt sind, zeigt die folgende Zusammenstellung der Namen nach inhaltlichen Kriterien.

\section{Landschaft und landwirtschaftliche Nut- zung im Spiegel der Flurnamen}

Die Hochgebirgsorte Obergurgl und Vent sind ein Paradebeispiel für Sonderentwicklungen im Flurnamenschatz eines Gebietes (für Obergurgl vgl. auch Ortner 2009: 466 ff.). Seit dem Hochmittelalter wird benannt, was landschaftlich auffällig und für die landwirtschaftliche Nutzung wichtig war.

„Flurnamengebung ist Feldgliederung. [...] Feldgliederung bedeutet [...] in erster Linie die Aufgliederung größerer räumlicher Flächen in kleinere ,Interessengebiete' und deren genaue Kennzeichnung durch In-Bezug-Setzen auf festgelegte, das heißt: benannte Richtpunkte. Flurnamengebung in diesem Sinne entspricht also im wesentlichen [sic!] dem Aufbau eines verbindlichen Koordinatensystems zur leichteren Fixierung von Punkten im Raum.“ (Bauer 1965: 251)

Die extreme Kleinräumigkeit und die ökologische Vielfalt der Umgebung (Bätzing 1991: 64, 284) sind besonders „namenträchtig“. Das belegen die vielen $\mathrm{Na}$ turnamen. Sie nehmen vor allem Bezug auf topographische Gegebenheiten:

(1) Landschaftsformationen (Berge, Täler, Felsen und andere Geländeformen)

Karlesspitze $_{\mathrm{OG}}$, Mitterberg ${ }_{\mathrm{VE}}$, Steintal ${ }_{\mathrm{OG}}$, Brunntal $_{\mathrm{VE}}$, Talegge $\mathrm{OG}$, Durchs Loch ${ }_{\mathrm{OG}}$, Soom $_{\mathrm{OG}, \mathrm{VE}}($ Saum $=$ '[...] fortlaufender Höhenrand, der Saum einer eiszeitlichen oder tertiären Hochfläche'; TONK: 799)

Oft benennen Flurnamen mit -stein und -eck besondere „Merkzeichen“ in der den Menschen umgebenden Welt (vgl. Lynch 1968, 20). Diese haben vor allem Orientierungs- oder Warnfunktion:

Hirtenstein $_{\mathrm{OG}}$, Am Spitzigen Stein ${ }_{\mathrm{OG}}$ (s. Abb. 1), Fauler Stein ${ }_{\mathrm{VE}}$, Fuchsater Stein ${ }_{\mathrm{VE}}$ ('fuchsroter Stein'), Hohler Stein ${ }_{\mathrm{VE}}$ ' Großer Stein ${ }_{\mathrm{VE}}$, Hockender Stein ${ }_{\mathrm{VE}}$ (vgl. Plangg 1998/2011)

Sommeregge $_{\mathrm{OG}}($ Egge = 'vorstehendes Ende im Gelände'; WTM: 141), Rauhegge $_{\mathrm{OG}}$, Jungegge ${ }_{\mathrm{OG}}$, Sackegge ${ }_{\mathrm{VE}}$, Lehneregge $_{\mathrm{VE}}$, Murentalegge $\mathrm{VE}$

Die Orientierungsfunktion wird direkt angesprochen in Zeigerle ${ }_{\mathrm{OG}}$ oder 
Zeigerköfele $_{\mathrm{VE}}$ (Zoager $=$ 'Steinzeichen'; WTM: 718). Markante Punkte in der Landschaft werden auch bildlich hervorgehoben:

INT-P: Und vorher, den Hång då oubm. Hoaßt man's Beim Weißn Mandlan ${ }_{\mathrm{OG}}$. Weil [...] wenn man iatz då inhn geaht und links auchnschaut, vor man zu dem Teich kimmt, siecht man a sou an Fels, an groaßn Stoan. Und då ischt sou a weißr [...], gånz weißr Stoan drinn. Und deswegen hàt man's då „Ban Weißn Mandlan" gehoaßn.

INT-P: ,Und vorher, den Hang da oben nennt man Beim Weißen Männlein ${ }_{\mathrm{OG}}$. Weil [...] wenn man jetzt dort hineingeht und links hinaufschaut, bevor man zu dem Teich kommt, sieht man so einen Felsen, einen großen Stein. Und da ist so ein weißer [...], ganz weißer Stein drin. Und deswegen hat man's dort „Beim Weißen Männlein “genannt.'

(2) Lage

Auf den Bichelen ${ }_{\mathrm{OG}}$ - In der Rinne ${ }_{\mathrm{VE}}$; Obere Brosers Aue ${ }_{\mathrm{OG}}$ - Mittlere Wendls Aue $_{\mathrm{OG}}$ - Untere Brosers Aue ${ }_{\mathrm{OG}}$ (Broser, Wendl = Hofnamen); Innerster Soom - Mittlerer Soom ${ }_{\mathrm{VE}}$ - Oberster Soom $\mathrm{VE}$ (Soom = 'fortlaufender Höhenrand'; TONK: 799); Mitterbach ober dem $W_{\text {eg }}$ - Mitterbach unter dem Weg (Abb. 7).
Es gibt eine Reihe von Flurnamen, die den eigentlichen Namen aussparen, sogenannte Metonymien. Hier übernehmen die Positionsangaben gleichzeitig die Bezeichnungsfunkti-

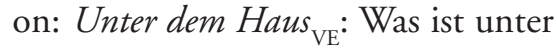
dem Haus? Eine Wiese, ein Hang oder eine Rinne? Ähnlich:

Neben der Nasenwand ${ }_{\mathrm{OG}}$ Bei den Bachlen $_{\mathrm{VE}}$, Am See ${ }_{\mathrm{VE}}$, Zwischen den Bächen $_{\mathrm{OG}}$ (Abb. 8)

\section{(3) Dimension und Größe}

Selten wird nach der Dimension differenziert, z.B. Tiefes Tal ${ }_{\mathrm{VE}}$. Das Wort hoch kommt meist in der Bedeutung 'hochgelegen' vor, verweist also auf eine Lage, s. (2). Hoch ist dann nicht als Gegensatz zu tief zu verstehen, vgl. die lagebezeichnenden Varianten Hoher Faichtwald und Oberer Faichtwald ${ }_{\mathrm{VE}}$ für dasselbe Stück Wald (Faicht $/$ Faichte = 'Fichte'; WTM: 170).

Wesentlich häufiger wird auf die Größe einer Flur Bezug genommen, z.B. Großes $K_{\text {aG }}$, Kleiner Waldstein ${ }_{\mathrm{VE}}$, Kleinalpe ${ }_{\mathrm{OG}}$. Die exzessive Verwendung der Nachsilbe -le (= -lein) in den sogenannten Diminutiva ist ein Indikator für die durch natürliche Gegebenheiten und durch ständige Besitzteilung entstandene Kleinund Kleinstgliederung der Fluren. 


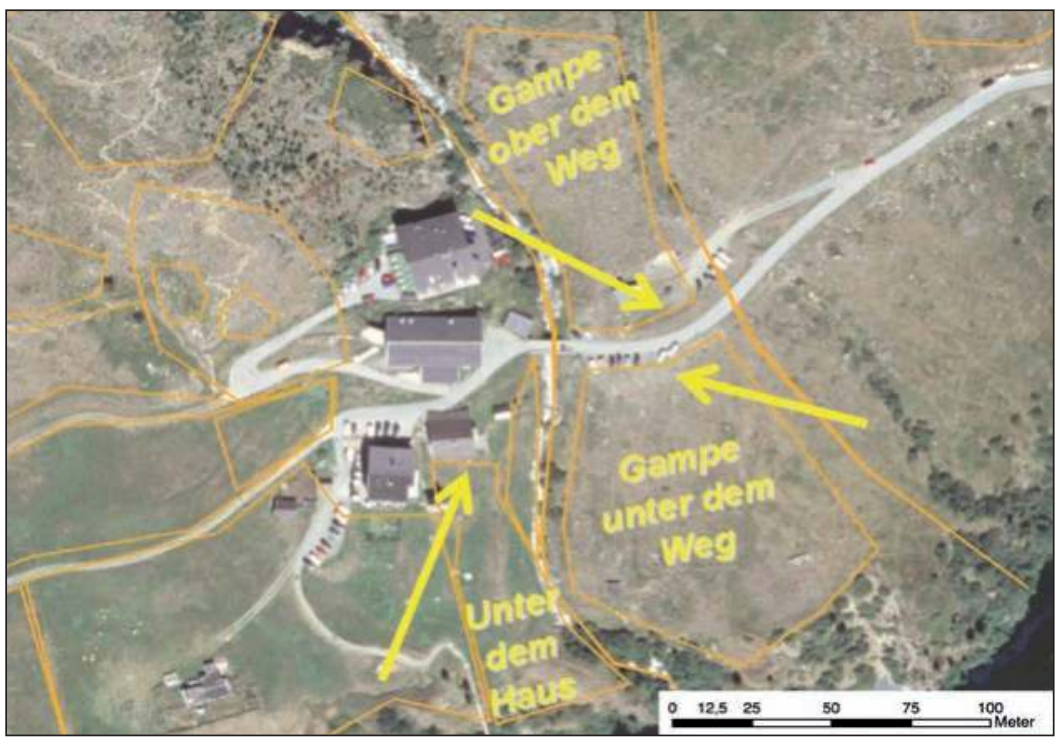

Abb. 7:

Positionsangaben als Flurnamen(bestandteile): Gampe unter dem $W_{\text {VE }}$, Gampe ober dem Weg ${ }_{\mathrm{VE}}$, Unter dem Haus $_{\mathrm{VE}}$ (Gampe = 'ebener freier Platz bei der Alphütte'; WTM: 201). (Orthofoto: BEV, Befliegung 2003, Rofenhöfe bei Vent)

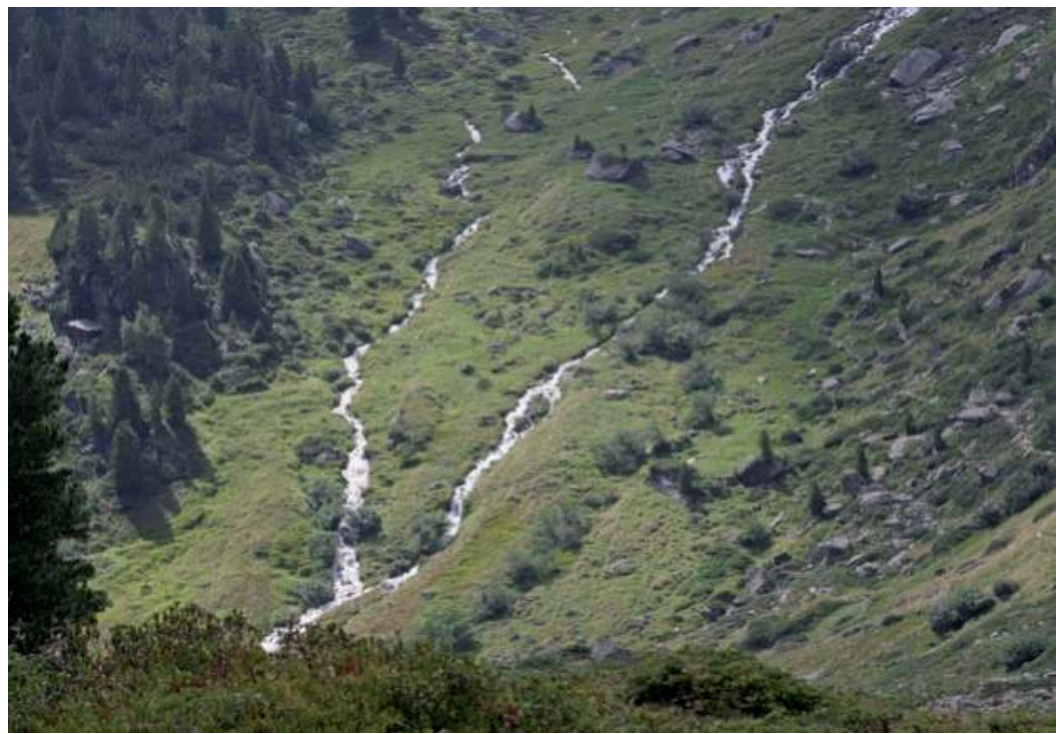

Abb. 8:

Metonymien: Die Flur wird über den Umweg der Positionsangabe benannt: Zwischen den Bächen.

(Foto: R. Kaufmann) 
17-20\% der Flurnamen enden auf -le. Hier ein Ausschnitt aus dem Reigen der Verkleinerungsformen: Äuele $_{\mathrm{OG}}$ ('kleine Au'), Müblbachle ${ }_{\mathrm{OG}}$ ' Bichele $_{\mathrm{OG}}$, Gartle $\mathrm{OG}_{\mathrm{OG}}$, Jöchle $\mathrm{OG}_{\mathrm{OG}}$, Karle $\mathrm{OG}$ ('kleines Kar'), Küppele ${ }_{\mathrm{OG}}$ ('kleine Kuppe'), Unteres Legerle ${ }_{\mathrm{OG}}$ ('kleiner Lagerplatz für Vieh'), Pirchmösle ${ }_{\mathrm{OG}}$ ('kleines Moor mit Birken'), Rastbödele ${ }_{\mathrm{OG}}$, ÄuBeres Schwindeleggele ${ }_{\mathrm{OG}}$, Spitziges Köfe$l_{\mathrm{OG}}$, Wurmkögele ${ }_{\mathrm{OG}}$, Zweckle $\mathrm{OG}_{\mathrm{OG}}$ ('kleiner Zwickel'); Ackerle $_{\mathrm{VE}}$, Eisbachle $\mathrm{VE}_{\mathrm{VE}}$, Oberstes Bödele $\mathrm{VE}_{\mathrm{VE}}$ ' Hintereisbrüggle $_{\mathrm{VE}}$, Martins Ebenle ${ }_{\mathrm{VE}}$, Hochgample $_{\mathrm{VE}}$ ('hochgelegene kleine Gampe' = 'ebener freier Platz bei der Alphütte'; WTM: 202), Grüble ${ }_{\mathrm{VE}}$ ('kleine Grube'), Hörnle ${ }_{\mathrm{VE}}$, Schwarzköfele ${ }_{\mathrm{VE}}$, Eiskögele $_{\mathrm{VE}}$, Mösle $_{\mathrm{VE}}$, Loamle ${ }_{\mathrm{VE}}($ Loam = 'Lehm'; WTM: 369), Dunkelrinnele ${ }_{\mathrm{VE}}$ ' Waldele $_{\mathrm{VE}}$, Wiesle $\mathrm{V}_{\mathrm{VE}}$

Wie gering die Ausmaße von Fluren sein können, die mit solchen Verkleinerungsformen benannt werden, zeigt Abb. 9.

Die Lage einer Flur bzw. ihre räumliche Gestalt waren offenbar vorrangige Bezeichnungsnotwendigkeiten für Hirten und Bauern. Die große Vielfalt an raumbezogenen Namen lässt sich durch die reich gegliederte Hochgebirgslandschaft mit ihren vielen Gefahren und kargen Nutzungsmöglichkeiten erklären.

Außer auf topographische Gegebenheiten beziehen sich Flurnamen auch auf andere Phänomene der Umwelt und auf den landwirtschaftlichen Alltag, allerdings wesentlich seltener. Die folgenden Benennungsmotive beleuchten spotlightartig verschiedene andere Elemente des Lebensraumes:

\section{(4) Bodenbeschaffenheit} $A_{m}$ Moos $_{\mathrm{OG}}$ ('Moor'), Schwemmploder ${ }_{\mathrm{OG}}$ (Ploder = 'Sumpf'; WTM: 91), Brunnenboden $_{\mathrm{VE}}$ (INT-P: ,Da sind mehrere Quellen.'), Loamtal ${ }_{\mathrm{VE}}$ ('Lehmtal'), Steiniger Taufen ${ }_{\mathrm{VE}}$ (Taufen = 'Gleitbahn, -rinne für Stämme'; WTM: 632; WBÖ 4: 1077), Steinige Rinne ${ }_{\mathrm{OG}}$

\section{(5) Naturgefahren}

Flurnamen, die auf steiles, abschüssiges Gelände Bezug nehmen, können indirekt Naturgefahren signalisieren, wie das z.B. im bildlichen Namen Höllegge $_{\mathrm{OG}}$ der Fall ist, s. auch (1). Direkt genannt werden Lawinen und Muren, Naturgefahren, die auf 2000 - 3000 m Seehöhe immer präsent sind:

Windlehne $_{\mathrm{OG}}$ ('Ort, wo Staublawinen abgehen'; vgl. WTM: 706), Lehnereg$g e_{\mathrm{VE}}$, Murental $_{\mathrm{VE}}$

\section{(6) Gewässer}

Weißer Bach ${ }_{\mathrm{OG}}$, Mahdtalbachle ${ }_{\mathrm{VE}}$, Honnesen Brünnle $\mathrm{VE}_{\mathrm{VE}}$, See ${ }_{\mathrm{VE}}$, Nasensee $_{\mathrm{OG}}$, Auf Krumpwasser $_{\mathrm{OG}}$ (krump = 'krumm'; INTP: Und dås ischt wåhrscheinlich, weil då ischt danebm a Boudn und då rinnt des Wassr fascht in an sou an Bougn rund 

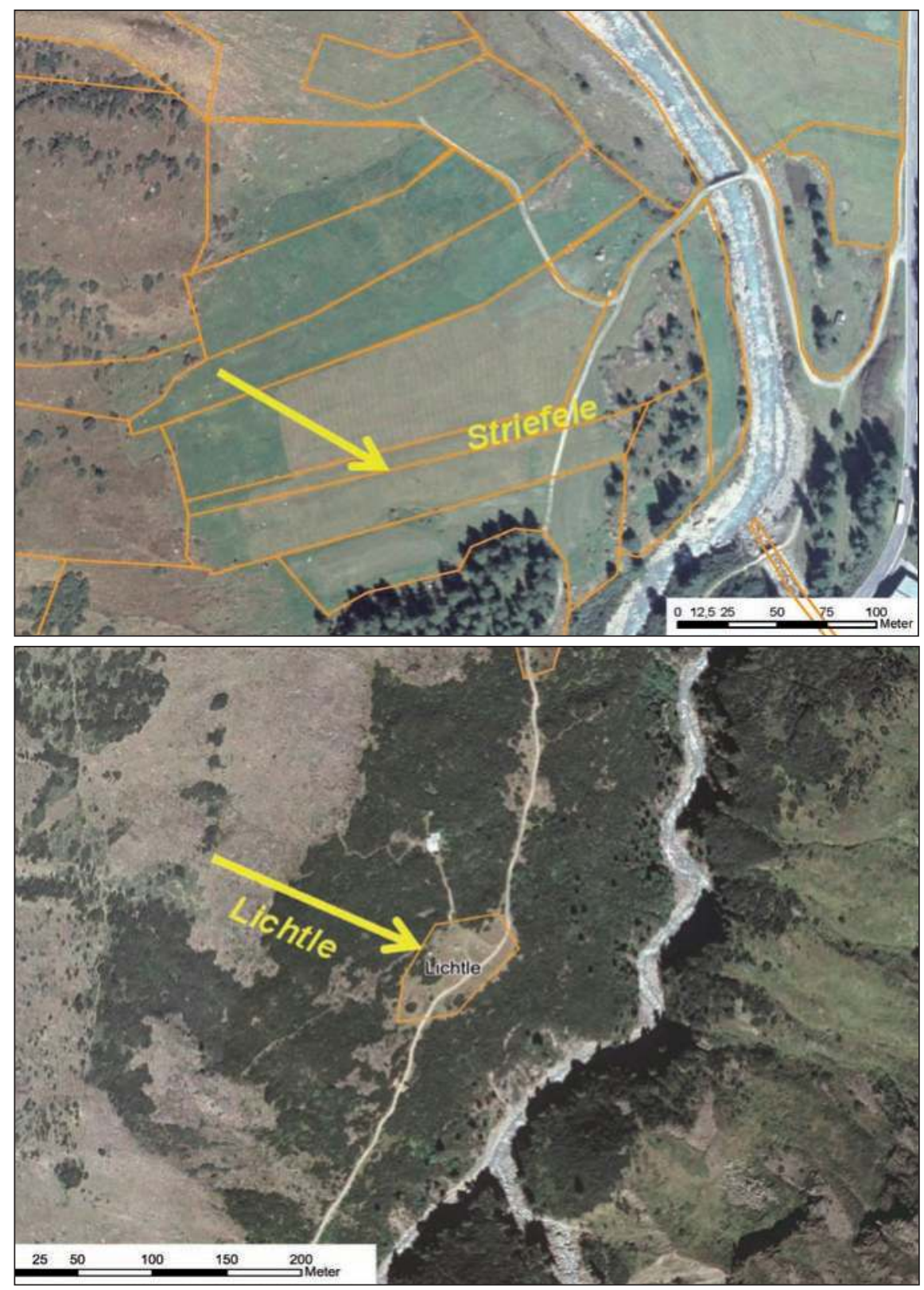

Abb. 9:

Flurnamen, die auf Dimensionen Bezug nehmen: Striefele ${ }_{\mathrm{OG}}$ benennt eine sehr schmale Flur (WTM: 612), eben nur ein 'Streifchen' (a). Lichtle ${ }_{\mathrm{VE}}$ benennt eine 'kleine Lichtung' im großen Wald (vgl. WTM: 390) (b). (Orthofoto: BEV, Befliegung 2003) 
ummat [...]. INT-P: ,Und das ist wahrscheinlich [deswegen], weil da daneben eine Bodenfläche ist und da rinnt das Wasser fast in so einem Bogen rund herum $\left.[\ldots]^{c}\right)$

Das Benennungsmotiv [Gewässer] liegt übrigens auch dem Namen Gurgl $l_{\mathrm{OG}}$ zugrunde: 'gefährliche Wasserwirbel in tiefen Gewässern' (TONK: 791, 796, 822, 840).

Manche Flurnamen sprechen indirekt ökologische Vorbedingungen für die Berglandwirtschaft an. Nederboden ${ }_{\mathrm{OG}}$ z.B. benennt eine schattseitige Flur. Daraus folgt, dass sie nicht ideal nutzbar ist. Daneben gibt es viele Namen, die sich ganz direkt auf verschiedene landwirtschaftliche Gegebenheiten beziehen. Sie geben Auskunft über Mahd, Alm- und Weidewirtschaft:

(7) (Alp-)Weiden, Wiesen, Mahdflächen und Wälder

Oberes Feld ${ }_{\mathrm{OG}}$ ('Wiese'), Leite ${ }_{\mathrm{OG}, \mathrm{VE}}$ ('ansteigendes Feld, Feldhang'; WTM: 384), Anger $_{\mathrm{OG}, \mathrm{VE}}$ ('umzäuntes Wiesfeld beim Haus, im Wald'; WTM: 25), Äußere Wiese $_{\mathrm{VE}}$, Stableinmähder ${ }_{\mathrm{VE}}$, Bergmahd Plattai $_{\mathrm{VE}}$, Wendls Mittlere Au $u_{\mathrm{OG}}$, Jörgeles Wald $_{\mathrm{OG}}$, Waldele

Einige Namen geben Aufschluss über die Nutzbarmachung von Land in früherer Zeit. Es sind entweder allgemeine Rodungsnamen (mit roden zusammenhängend) oder Flurnamen, die spezifisch auf das Urbarmachen von Flächen durch Brandrodung verweisen:

Anraitle $_{\mathrm{OG}}$ ( $<$ mhd. riute = 'Stück Land, das durch riuten urbar ist gemacht worden'; Lexer 1872-1878, 2: 471), Raut $_{\mathrm{OG}}$ ('Raut, Rodung'; WTM: 475); Brand ${ }_{\mathrm{OG}}$ ' Brandle $_{\mathrm{VE}}$, Brandschrofen ${ }_{\mathrm{VE}}$, Brenner ${ }_{\mathrm{OG}}$, Martins Brennbichl $_{\mathrm{VE}}$, die Brinsten ${ }_{\mathrm{VE}}$ (Brinsten Plural $<$ mhd. brunst, Plural brünste $=$ 'das Brennen, Brand')

(8) Pflanzen

Einerseits werden Bewuchsverhältnisse, die ausschlaggebend für gute oder schlechte Beweidungs- bzw. Mahdbedingungen sind, direkt thematisiert, z.B. Am Inneren Barst ${ }_{\mathrm{OG}}$ ('mit Borstgras bewachsene Fläche'), Distelwiese $_{\mathrm{OG}}$. Anderseits geben Pflanzenbezeichnungen in Flurnamen auch einfach nur Hinweise auf landschaftliche Eigenheiten, z.B. Zum Larch ${ }_{\mathrm{OG}}$ ('Bei der Lärche'), Zirbenwand $_{\mathrm{VE}}$, Rumsoppen $\mathrm{OG}$ ('Bärlauchhügel'; zu Rams = 'Bärenlauch' [sic!] und Oppen = 'runder Bichl'; TONK: 798).

Eine Besonderheit bei Flurnamen mit Pflanzenbezeichnungen sind Kollektivbezeichnungen mit den dialektalen Nachsilben - at bzw. -it sowie -ach. Sie fassen eine bestimmte Menge/,Vielheit“ zusammen:

Pirchat $_{\mathrm{VE}}$ / Pirchitt ${ }_{\mathrm{OG}}$ : 'Ort mit (vielen) Birken'; das Salchach ${ }_{\mathrm{OG}}$ : 'Ort mit (vielen) Weiden' ( $<$ die Salche = 'Salweide'; 
WTM: 502), Ebners Ronach ${ }_{\mathrm{OG}}$ : 'Ort mit (vielen) umgestürzten Bäumen' (< die Rone = 'umgefallener verdorrter Baum'; WTM: 490), das Poschach ${ }_{\mathrm{OG}}$ (der Poschn = 'junges Nadelholz, Jungwald, Fichtensetzling'; WTM: 99), das Zetach ${ }_{\mathrm{OG}}$ (die Zete = 'niederer Strauch, Gestrüpp, Heiderich, Alpenrosenstrauch, Legföhre, Beerenstrauch'; WTM: 726; in Obergurgl und Vent aber überwiegend: 'Grünerle').

An dieses Muster der Kollektivbezeichnungen schließen auch andere Grundwörter an, und zwar solche aus den oben genannten Kategorien (1) und (4), vgl.

Putzach $_{\mathrm{OG}}$ (Putz< althochdeutsch puzza $<$ rom. * putëu < lat. puteus = 'Brunnen, Pfütze’; vgl. TONK: 911; WTM: 123; bzw. direkt aus rom. ${ }^{*}$ puts $(o)<{ }^{*}$ putju; WBÖ 3: 1568 f.), Brunnach ${ }_{\mathrm{OG}}$ : 'Gebiet mit (vielen) Quellen', Plattach ${ }_{\mathrm{OG}}$ : 'Gebiet mit Steinplatten' (WTM: 86; mit den Varianten In den Platten $_{\mathrm{OG}}$, Platt $_{\mathrm{OG}}$ und Plattai $_{\mathrm{VE}}$; s. Tab. 3, S. 49), Steinmaurach $_{\mathrm{OG}}$ : 'Gebiet mit (vielen) Steinen' (Maurach $=$ 'Steingerölle von Bergstürzen'; WTM: 420)

\section{(9) Tiere}

Tierbenennungen werden in Flurnamen sehr selektiv eingesetzt. Viele scheinen nie auf (z.B. Murmeltier), wahrscheinlich weil sie nicht hervorhebenswert sind. Flurnamen mit Tierbezeichnungen verweisen haupt- sächlich auf Nutztiere, in geringerer Zahl bezeichnen sie Wild oder Tiere als Gefahrenpotentiale:

Rinderköfele $_{\mathrm{OG}}$, Ochsenkopf $\mathrm{OG}_{\mathrm{OE}}$, Stierbichl $_{\mathrm{OG}}$, Kubberg $_{\mathrm{VE}}$, Kälberpuit ${ }_{\mathrm{VE}}$ (Puite $=$ 'eingehegtes Grundstück'; vgl. Haslinger 2009: 236), Kitzleite ${ }_{\mathrm{OG}}$, Geißköfele ${ }_{\mathrm{OG}}$, Oberer Schafleger $_{\mathrm{VE}}$ (Leger = 'Lager, Lagerplatz für das Vieh auf der Alpe, Weide'; WTM: 381); Hirschköfel ${ }_{\mathrm{VE}}$, Fuchsgande $_{\mathrm{VE}}$ (Gande = 'Hang mit Steingeröll'; WTM 203); Wurmegge OG (INT-P: ,[Da] gibt es Kreuzottern und Blindschleichen.')

(10) Nutzung

Viele Flurnamen spiegeln typische berglandwirtschaftliche Tätigkeiten wider, s. auch (11):

Mittermahdl ${ }_{\mathrm{VE}}$ ('mittlere kleine Mahdfläche'), Mahdstubl ${ }_{\mathrm{OG}}$ (bildlich), Ötzrain $_{\mathrm{OG}}($ etzen $=$ 'Vieh weiden nach der [Heu-]Ernte'; WTM: 152; vgl. auch Ötztal), Hirtenstein ${ }_{\mathrm{OG}}$ ('Rastplatz für Hirten')

(11) Gebäude/Gewerbeanlagen

Bienenhaus $_{\mathrm{OG}}$, Alte Hütte ${ }_{\mathrm{OG}}$, Jagdhüt$t e_{\mathrm{OG}}$, Mitterbachpille $_{\mathrm{VE}}$ (Pille $=$ 'Heuhütte'), Beim Stadele ${ }_{\mathrm{OG}}$, Rossstall ${ }_{\mathrm{OG}}$, Mühle $_{\mathrm{OG}}$, Kaser ${ }_{\mathrm{VE}}$ ('Alphütte, in welcher gesennt wird'; WTM: 327)

\section{(12) Wege, Übergänge und Grenzen}

Beim Steg ${ }_{\mathrm{OG}}$, Schienenweg ${ }_{\mathrm{OG}}$ (betrifft das Heuziehen auf Holzschienen), Alter Sennweg $_{\mathrm{VE}}$, Hohe Brücke ${ }_{\mathrm{OG}}$, March $_{\mathrm{VE}}$ 
('Kennmarke, Kennzeichen, Grenzzeichen, Feldgrenze'; WTM: 415), Zaun $_{\mathrm{VE}}$

\section{(13) Besitzer/Nutzer}

Die (heute noch oder bis vor kurzem) bewirtschafteten Fluren sind sehr kleine Einheiten. Dies ist einerseits durch die landschaftlichen Gegebenheiten erklärbar, vgl. (1) - (3). Andererseits gibt es auch besitzrechtliche Gründe. Über Jahrhunderte hinweg wurden Mahdflächen und Weiden geteilt und damit die Besitzverhältnisse neu geregelt. Dann mussten auch für die kleinsten Einheiten Namen geschaffen werden - und dies geschah oft, indem der Besitzer bzw. Nutzer im Flurnamen genannt wurde. Das Benennungsmotiv [(ehemaliger) Besitzer/Nutzer] ist fast so wichtig wie das Benennungsmotiv der Positionierung einer Flur im Raum (s. (2) Lage)

Auf (ehemalige) Besitzer verweisen vor allem alte Hofnamen, die sich aus Rufnamen entwickelt haben (Ortner 2009: 476-479) (Abb. 10): Brosers Auenrain ${ }_{\mathrm{OG}}$ (< Ambrosius), Dodls Grünebene ${ }_{\mathrm{OG}}(<$ Methodius $)$, Wendls Barst ${ }_{\mathrm{OG}}(<$ Wendelin), Jörgeles Infangle ${ }_{\mathrm{OG}}$ ('kleiner Einfang', 'umzäuntes Stück im Feld, Wald'; WTM: 311), Jakobn Anger ${ }_{\mathrm{OG}}$, Vennegilds Angerle ${ }_{\mathrm{OG}}$, Kleons Ebenle ${ }_{\mathrm{VE}}$, Martins Ebenle ${ }_{\mathrm{VE}}$, Piusen Hütte ${ }_{\mathrm{VE}}$, Seraphins Infang ${ }_{\mathrm{VE}}$
Seltener sind Flurnamen, die mit Familiennamen gebildet werden, z.B. Gamper Moos ${ }_{\mathrm{OG}}$, Grüner Grünebene $_{\mathrm{OG}}$, Scheiberbichl ${ }_{\mathrm{OG}}$, Pirpamer Steiniglehn $n_{\mathrm{VE}}$

Eine Eigenheit der sehr kleinen Ortschaft Vent mit dem Weiler Rofen ist die „egozentrische“ Besitzerperspektive im Flurnamensystem, wobei diese Flurnamen einen sehr engen Gültigkeitsbereich haben (vgl. Windberger-Heidenkummer 2001: 232 ff.):

Unser Bödele $_{\mathrm{VE}}-$ Nachbars Bödele $_{\mathrm{VE}}$; Unsere Grüblesköfelen ${ }_{\mathrm{VE}}-$ Nachbars

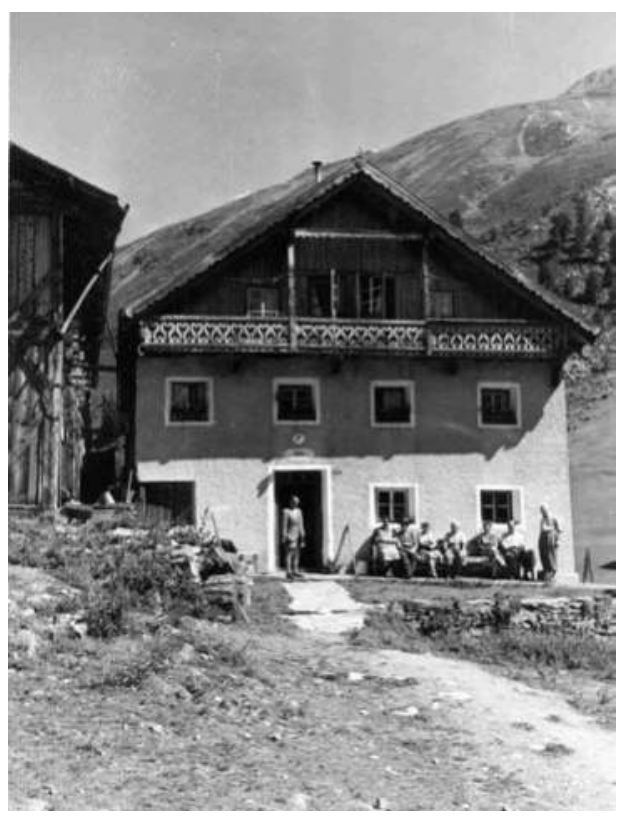

Abb. 10: Elternhaus der Familie Grüner, der Martinshof. Mit freundlicher Abdruckgenehmigung von Annemarie Schöpf (geb. Grüner) (Foto: Fam. Grüner). 
Grüblesköfelen $_{\mathrm{VE}}$; Unser Neder ${ }_{\mathrm{VE}}-$ Nachbars Neder $_{\mathrm{VE}}($ Neder $=$ 'Schattseite')

Die Perspektive kann je nach Sprecher variieren und damit auch die Referenz der komplementären Flurnamenpaare: Der Nachbar bezeichnet seinen Grund von seiner Perspektive aus ebenfalls als Unser Bödele, und das, was vorher Unser Bödele geheißen hat, wird dann eben Nachbars Bödele.

\section{(14) Bewertungen und Einstellungen}

Im Zusammenhang mit den Nutzungsmöglichkeiten werden in den Flurnamen - sehr selten - auch Wertungen ausgedrückt, die Einstellungen zu einzelnen Landschaftselementen, Einschätzungen über ihre Brauchbarkeit für landwirtschaftliche Zwecke oder Warnungen vor Gefahrenpotentialen direkt sichtbar machen:

Rauher Bichl ${ }_{\mathrm{OG}}$, Schlechter Boden ${ }_{\mathrm{VE}}$, die Schöne $_{\mathrm{OG}}$ ('schöner, freier Fleck, schöner Wiesfleck'; TONK: 493), Schönwiese $_{\mathrm{OG}}$ ('schöne, gut nutzbare Wiese'), Beim guten Wasserle ${ }_{\mathrm{OG}}$, In der Hölle ${ }_{\mathrm{OG}}$, Bösegge ${ }_{\mathrm{OG}}$, Schiechegge ${ }_{\mathrm{OG}}$ (schiech = 'hässlich'; WTM: 521. Der Name bezeichnet eine Kurve, die bei Schnee oder Nässe für das Vieh gefährlich werden kann.)

Erst im Kommen sind ironisierende „Neonamen“ wie Hungerbichl ${ }_{\mathrm{OG}}$ (für ein Grundstück, auf dem ein großer Hotelkomplex steht). Doppelbenennung aus pragmatischen Gründen liegt auch vor, wenn neue touristische Bergnamen entstehen, z.B. Zahme Anna ${ }_{\mathrm{OG}}$ (für Annako$\mathrm{gel}_{\mathrm{OG}}$ ) oder Wilde Hilde (für Hohe Wilde $_{\mathrm{OG}}$ ), oder wenn „Schisportnamen" althergebrachte Flurnamen verdrängen, z.B. Rippenbrecher ${ }_{\mathrm{OG}}$ statt Am Wams ${ }_{\mathrm{OG}}$. Solche Brüche in den Benennungstraditionen sind ein Indiz für den tiefgreifenden soziologischen Wandel von der Berglandwirtschaft zum Tourismus.

Die Zusammenstellung der 14 Benennungsmotive in den Flurnamen von Obergurgl und Vent zeigt das Bild einer eigenständigen kleinräumigen Namenlandschaft („onymische ,Kleinlandschaften“", Hellfritzsch 1990/2010: 26). Ob diese Namenlandschaft insgesamt typisch für hochalpine Räume ist, wäre in einer umfassenderen Untersuchung zu klären.

\section{Flurnamengeschichten: Erfahrungs- wissen über hochalpine Räume und ihre Nutzung}

Die Interviews haben gezeigt, dass sich die Methode der Flurnamenerhebung hervorragend als Ausgangspunkt für Alltagsgeschichten über das bäuerliche Le- 
ben eignet. In natürlichen Gesprächssituationen erzählen die Interviewten über die hochalpine Naturlandschaft und deren Nutzung in Vergangenheit und Gegenwart. Flurnamen sind starke Impulse für Erinnerungen. Die erhobenen Flurnamengeschichten sind somit auch ein Beitrag zur Erhaltung des immateriellen kulturellen Erbes.

Die folgenden Beispiele sollen abschließend kaleidoskopartig einige wichtige Themen von „Flurnamengeschichten“ beleuchten: „Die Einschätzung von Nutzbarkeit und Qualität einer Flur“, „Viehwirtschaft und Beweidung“, „Mahd und Heutransport" sowie „Der Wandel in der bergbäuerlichen Gesellschaft“.

Die Einschätzung von Nutzbarkeit und Qualität einer Flur

Trockene Wiesen: Då mahnsch zwår, åbr es steht hinter dir wiedr auf.

INT-P 1: Sou a trockene Seitn! Då hommse frier oft - då gehn jå de Waale durch zum Bewässern - wenn's gånz trockn wår, håmmse oft extra zerscht in Wål ånlåssn, dass die Seite bewässert weard, dassn se mahn kennen überhaupt. Und heit numma sou årg, bleibt er stian oanfäch. Weard woll a Schåfkemmn.

INT-P 1: ,So eine trockene Seite [ist das]! Da haben sie früher oft - dort gehen ja die Waale [= Bewässerungskanäle] durch zum Bewässern - wenn es ganz trocken war, haben sie extra zuerst den Waal angelassen, dass die Seite bewässert wird, dass sie überhaupt mähen können. Und heute [ist das] nicht mehr so arg, da bleibt [das Gras] einfach stehen. Wird schon ein Schaf kommen [und es fressen].

INT-P 2: [...] des Egg', des håm mir untranåndr, dr Papa, allm die "Truckne Seite“[...] gehoaßn. [...] Då wåchst eigntlich lei a Barcht und wenn's nit feicht gnuag ischt, fäscht nicht mähbår. Då mahnsch zwår, åbr es steht hinter dir wiedr auf.

INT-P 2: ,[...] dieses Eck, das haben wir untereinander, der Papa, immer die „Trockene Seite“ [...] genannt. [...] Da wächst eigentlich nur Borstgras und wenn es nicht feucht genug ist, [ist es] fast nicht mähbar. Da mähst du zwar, aber es steht hinter dir wieder auf.

\section{Viehwirtschaft und Beweidung}

\section{Beweidung: in die Schneaflucht gean}

INT-P: Die Gurgler håbm des Recht gehåbm, wenn's im Friejair zu lange 's Viech nit auslåssen håbm kinnt, håbm se gedirft [...] in die Schneaflucht geahn. Zwieslstoan, Seldn, $i$ gloub' sogàr bis Längenfeld außn, [...] håbm se gedirft's Viech då af die Woade treibm. [...] Und nåchn håt man gset: "In die Schneaflucht geahn“. (Abb. 11)

INT-P: ,Die Gurgler haben das Recht gehabt, wenn sie im Frühjahr das Vieh zu lange nicht auslassen haben können, durften sie [...] in die Schneeflucht gehen. [Nach] Zwieselstein, 
Sölden, ich glaube sogar bis Längenfeld hinaus [- das sind $27 \mathrm{~km} !-]$, [....] durften sie das Vieh da auf die Weide treiben. [...] Und dann hat man gesagt: „In die Schneeflucht gehen“.'

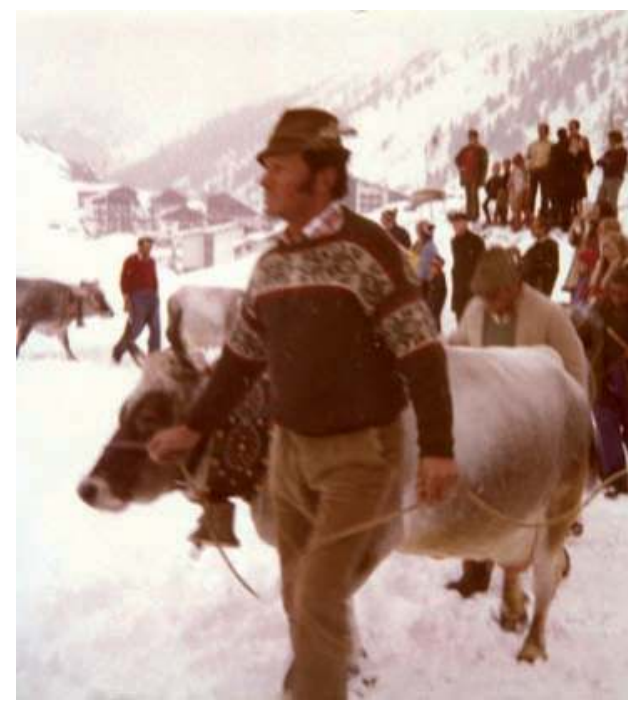

Abb. 11:

Viehwirtschaft. Mit freundlicher Abdruckgenehmigung der Familie Grüner (Foto: Familie Hansjörg Grüner, ca. 1974)

Viehtreiben über den Bach: Dånn håmmr ins in Schwainz vo die Kiah um die Hånd gewickelt - Die Eltern håbens nit wissen derfen.

INT-P: Und wenn man Rotmoostål drin, då isch jå gånz intn drin heit no koa Brett iwern Båch und mainchmål - rechts intn hoaßt man's Wåssrfällseite, weil då hint $d r$ Waissrfällgletschr isch, und links ebn Rotmoosseite - und wennes die Kiah iwern Båch gången sein, wenn er a sou gången ischt wia iatz, dån bische jå eigentlich nit dribrkemmen, $d r$ Båch hatt' di mit.
Dånn håmmr ins in Schwånz vo die Kiah um die Hånd gewickelt, wasch wo die Här sein, an Schwånz gehebt und iwern Båch onhn. Mir hein jå bårfuaß gewesn, wollten jå nit nass wearn. [...] Die Eltern håbens nit wissen derfen.

INT-P: ,Und wenn man [im] Rotmoostal drinnen [ist], da ist ja ganz hinten drin heute noch kein Brett über den Bach und manchmal - rechts drinnen nennt man es Wasserfallseite, weil da hinten der Wasserfallgletscher ist, und links eben Rotmoosseite - und wenn die Kühe über den Bach gegangen sind, wenn er [= der Bach] so verlaufen ist wie jetzt, dann bist du ja eigentlich nicht drübergekommen, der Bach hätte dich mit[gerissen]. Dann haben wir uns den Schwanz von den Kühen um die Hand gewickelt, weißt du, wo die Haare sind, am Schwanz festgehalten und [sind] über den Bach hinüber. Wir sind ja barfuß gewesen, wollten ja nicht nass werden. [...] Die Eltern haben das nicht wissen dürfen.

Viehtreiben im Schnee: Sel sein die ålten Tåtsachen.

INT-P: Wia mir sou Buabm warn in Kriag und gehiatet håbm, håmmr jå in gånzn Summr koan Schuah angelegt, in gånzen Summr bårfuaß gången. [...] INT: Des muaßja hårt gwesn sein untn [...] INT-P: Abr sou gsund fir die Fiaß! I hån nia kålte Fiaß. Ou in Wintr mitn Schikurs, hal gibt's ba mir nit. Und des wår gsund. [...] Na, mir håbm nia Schuah ankåbt. Und wenn mr dånn no sou iwer die Schneafeldr gången ischt, wo no Lawine wår. Des wår jå kålt, bårfuaß! Abr die Kiah håbm jå wås Wårms 
fälln gelàtt und då ischt man einigetretn. Jå, dånn wår's wårm. Und dainn ischt man wiedr a Drum schnell gången. Sel sein die ålten Tåtsachen. [...] Na, des ischt nit schlimm. Des ischt überhaupt nit schlimm, weil nåch zehn Mettr håsche die Fiaße wiedr hålbwegs saubr kåbt.

INT-P: ,Als wir so Buben waren in der Kriegszeit und gehütet haben, haben wir ja den ganzen Sommer keinen Schuh angezogen, den ganzen Sommer [sind wir] barfuss gegangen. [...] INT: ,Das muss ja hart gewesen sein unten $[\ldots]^{\prime}$ INT-P: ,Aber so gesund für die Füße! Ich habe nie kalte Füße. Auch im Winter [bei der Arbeit] mit dem Schikurs, das gibt's bei mir nicht. Und das war gesund. [...] Nein, wir haben nie Schuhe angehabt. Und wenn man dann so über die Schneefelder gegangen ist, wo noch [eine] Lawine war. Das war ja kalt, barfuss! Aber die Kühe haben ja etwas Warmes fallen gelassen und da ist man hineingetreten. Ja, dann war's warm. Und dann ist man schnell wieder ein Stück gegangen. Das sind die alten Tatsachen. [...] Nein, das ist nicht schlimm. Das ist überhaupt nicht schlimm, weil nach zehn Metern hast du die Füße wieder halbwegs sauber gehabt.'

\section{Mahd und Heutransport}

\section{Versorgung der Mäher mit Essen: Mittåg trågn}

INT-P: Wia mir no Kindr wårn, håm mir no miaßn Mittåg trågn. [...] Also de Måhder, dr Våtr und die Måhder, sein umma sechse odr wenn auchn, håm a Jause mitkåbt. Mittågs hàt man gekocht heruntn, då håmmr sou a Holzgschirr kåbt fir die Suppe, meischtns håt's eh Knedl gebm odr wås. Entwedr a Reisfleisch odr [...] Kråpfn odr an Apflstrudl odr hålt de zwoa Såchn. Und då håm mir als Kindr auhnmiaßn, mittågs, so schnell wia meglich, dass hål no wårm wår. Oft wår's eh schon kålt, bis mr oubm wårn. [...] Dånn håmmr wiedr oachageahn miaßn, die åndre Seite auchn, die Kiah houln. Und die Måhder und die sein hålt dånn umma siebene, achte żnåchts [...] kemmen. (Abb. 12)

INT-P: ,Als wir noch Kinder waren, mussten wir noch Mittag tragen. [...] Also, die Mäher, der Vater und die Mäher, sind gegen sechs [Uhr morgens] oder so ungefähr hinauf, [sie] haben eine Jause mitgehabt. Mittags hat man gekocht unten [im Tal], da haben wir so ein Holzgeschirr gehabt für die Suppe, meistens hat's eh Knödel gegeben oder [so] etwas. Entweder Reisfleisch oder [...] Krapfen oder einen Apfelstrudel oder eben die zwei Sachen. Und da haben wir als Kinder hinaufmüssen, mittags, so schnell wie möglich, damit [das Essen] halt noch warm war. Oft war's eh schon kalt, bis wir oben waren. [...] Dann haben wir wieder hinuntergehen müssen, [und] die andere Seite hinauf, die Kühe holen. Und die Mäher und die sind halt dann um sieben, acht abends [...] gekommen.'

Heutragen: Und a scheas Tragle, des isch der Stolz gewesn vo an Màhdar.

INT-P: So treit man a Tragle. [...] Und aso weart's aug'schobm, då setz i a Tiechl au, ja. Då 
Kapitel 2 | Die Landschaft und ihre Namen
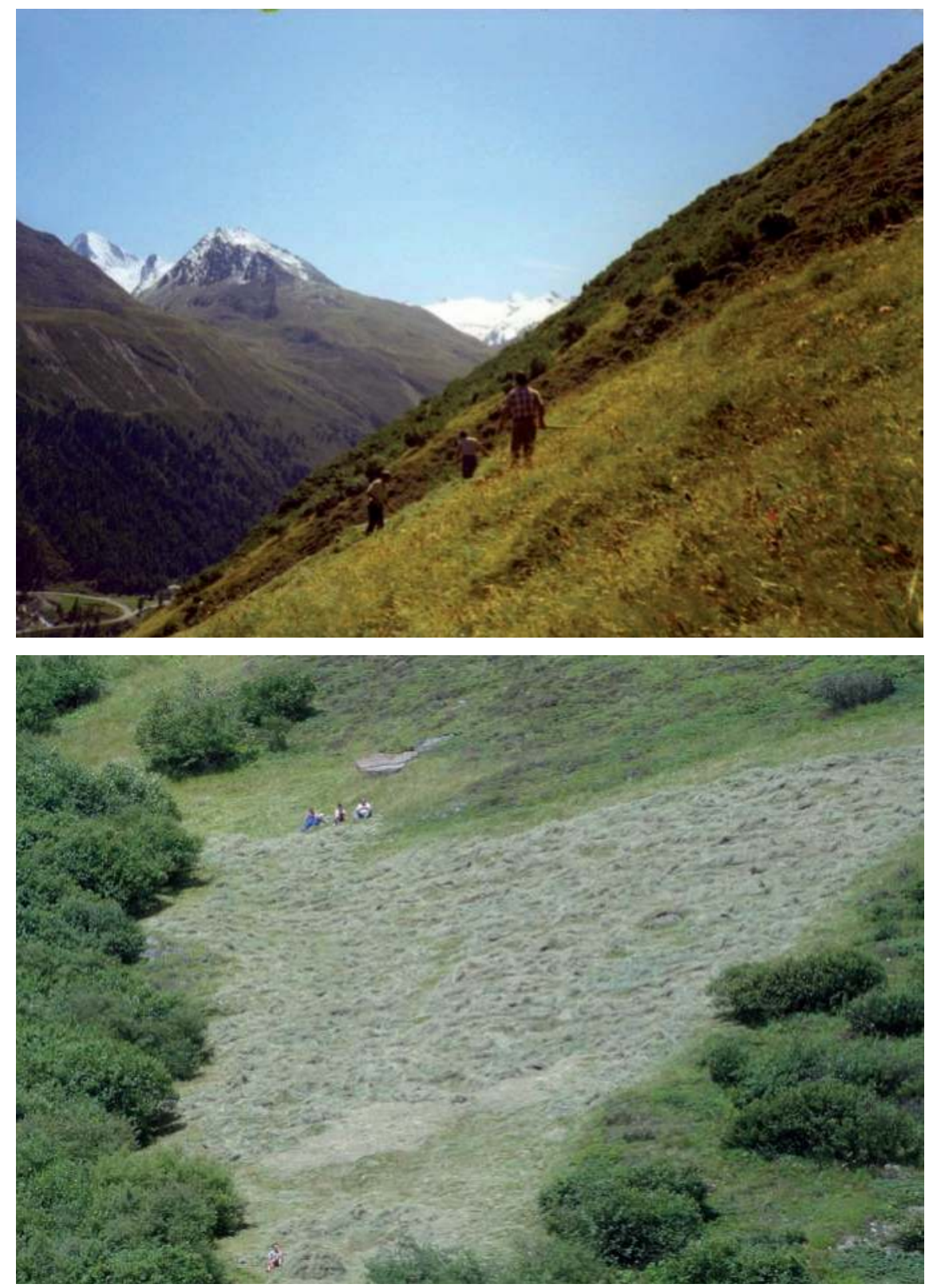

Abb. 12:

Oben: Beim Mähen im steilen Gelände (ca. 1974). Mit freundlicher Abdruckgenehmigung der Familie Grüner (Foto: Familie Hansjörg Grüner);

Unten: Rast beim Mähen auf Kitzlehn (2011). (Foto: R. Kaufmann) 
hån i no koa Glåtze, åber då hån $i$ mehr Hår, då isch's feinar. Und då isch aso ... so schuibt man au. So geaht man drunter inhn. Aso isch die Ferggla. Då isch's Kopfloch, und då schuibt man ietz hea. Auschiebm hàt man's gehoaßn. Jå, des isch alles vom Hatrogn. [...] Dås isch im indern Tåle dobm. 35, 40 Kilo. [...] Woasche wol. [...] 50. Und a scheas Tragle, wàs da då siesch, des isch der Stolz gewesn vo an Måhdar. [...] då tuat man schean årechn, dås weart gepflegt. (Abb. 13)

INT-P: ,So tragt man ein „Tragle“. [...] Und so wird es hinaufgeschoben, da ziehe ich ein [Kopf-]Tüchlein an, ja. Da hab ich noch keine Glatze, sondern da habe ich mehr Haare, da ist es feiner [auf dem Kopf zu tragen]. Und da ist so [...] so schiebt man [es] hinauf. So geht man darunter hinein. So ist die Ferggel. Da ist das Kopfloch, und da schiebt man jetzt her. Hinaufschieben hat man es genannt. Ja, das ist alles vom Heutragen. [...] Das ist im inneren Tal droben. 35, 40 Kilo [...] Weißt du wohl [...] 50 [Kilo Heu]. Und ein schönes "Tragle“ ist das, was du da siehst, das ist der Stolz eines Mähers gewesen. [...] da tut man [das Heu] gut abrechen, das wird gepflegt.'

Heute ist alles anders ...

Höhergelegene Weiden werden nicht mehr benutzt: Heit påckn's die Kiah nimmr und die Hirtn 0.

INT-P: Weil frier sein se mit de Kiah eigntlich bis zum Goaßbergfernr inhn gfärn fäscht. [...]
Heit påckn's die Kiah nimmr und die Hirtn 0.

INT-P: ,Weil früher sind sie mit den Kühen eigentlich bis fast zum Gaisbergferner hinein gegangen. [...] Heute packen es die Kühe nicht mehr und die Hirten ebenfalls [nicht].

Viel mehr Kühe waren auf der Weide: Ietz verhåltn si die Schåf a gånz ånders und's Rindviech sowieso.

INT-P: Aber hålt ietz a weniger, weil ietz durch des, weil då dia Abfährtn gerodet sein, [...] verhåltn si die Schåf a gånz ånders und 's Rindviech [...] sowieso. 'S isch jå nimmr sou viel Rindviech [...], frier [...] isch ma mit die Kiah, die [...] då, des Hoarnviech sågn mir, de man gemolchn håt, de Kiah, [...] de sein [...] untertågs a då gånz auffigången, weil jå viel mehr Kiah geweisn sein.

INT-P: ,Aber jetzt halt auch weniger, weil jetzt dadurch, dass da die Abfahrten gerodet sind, verhalten sich die Schafe auch ganz anders und das Rindvieh [...] sowieso. Es gibt ja nicht mehr so viel Rinder [...], früher [...] ist man mit den Kühen, die [...] da, das Hornvieh sagen wir, die man gemolken hat, die Kühe, [...] die sind [...] untertags auch da ganz hinaufgegangen, weil es ja viel mehr Kühe gegeben hat.

Die Murmeltiere folgen den Mähern ins Tal: Jå, natirlich sein de a nit bled.

INT-P: [...] frier håt man natirlich mehr gmahnt, de Flächn bis zum Felsn aufn alles - ietz mahnt man hålt 's Breckl untn. Wăhrscheinlich eh nimmr lång, weil die Murmeltie- 

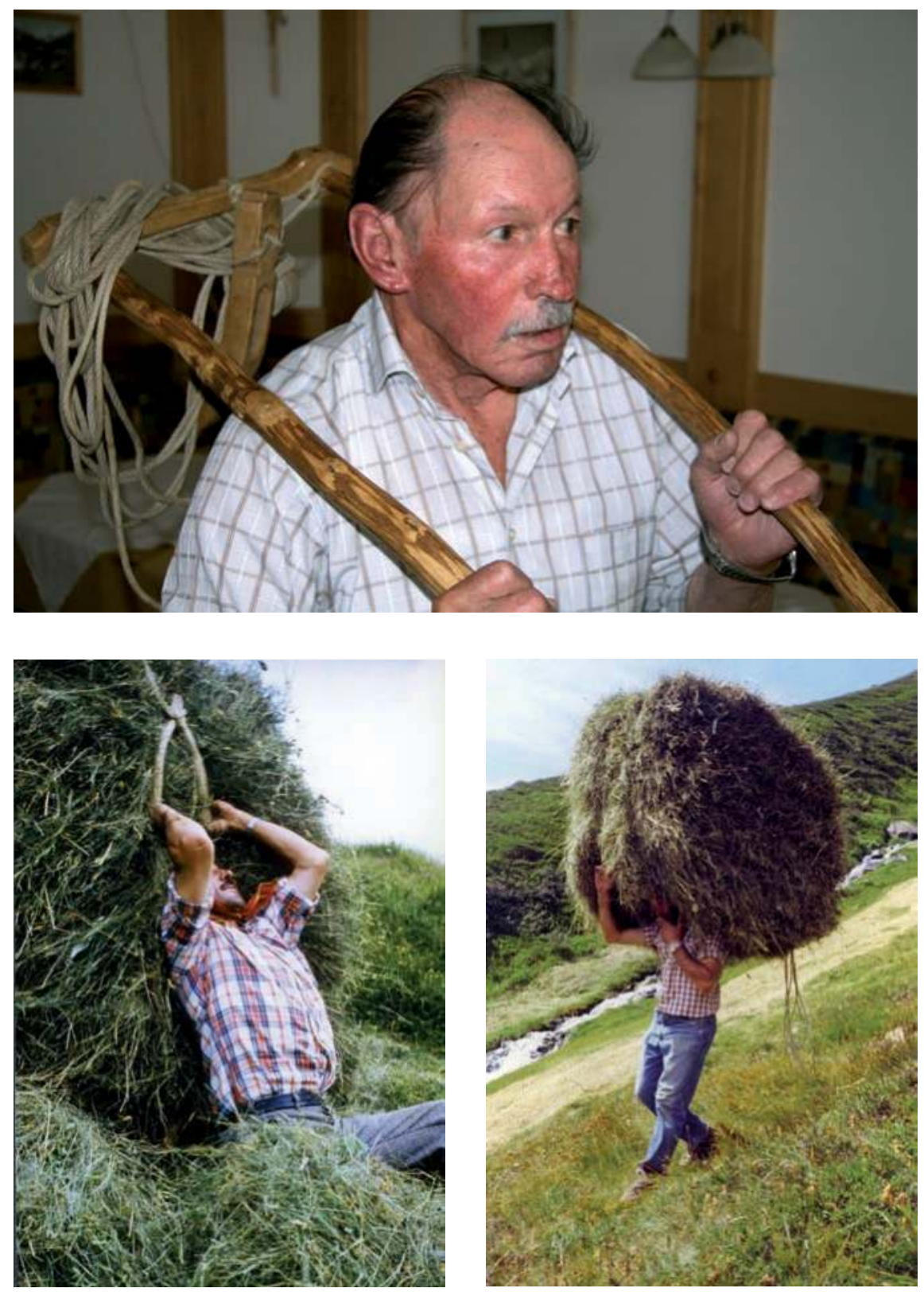

Abb. 13:

Oben: Hansjörg Grüner zeigt, wie man die Ferggel benützt. (Foto: R. Kaufmann, 2008);

Links und rechts (ca. 1974): Hansjörg Grüner „schiebt“ das Tragle „hinauf“ und trägt es ins Tal. Mit freundlicher Abdruckgenehmigung der Familie Grüner (Foto: Familie Hansjörg Grüner) 
re inzwischen den Besitz ergriffn hån. [...] In Lehnarle. Alle drei Mettr ischt a Murmltierloch schon. INT: Sant dia nu mehr woan? INT-P: Mehr wårscheinlich sein nit woarn. Frier hät man natirlich bis gånz oubm hin alls gmäht. Und de håm immr frisches Fuattr kåbt dånn. $J a ̊$, natirlich sein de a nit bled. De sein natirlich mit die Måhder ins Tål gången. [...] 'S frischere Fuatr håbm 's natirlich då. [...] Speziell, wenn då umme schaugsch zur Mittagszeit, in Barcht im Flåchn drübm, do sein gråd gnuag!

INT-P: ,[...] früher hat man natürlich mehr gemäht, die Flächen bis zum Felsen hinauf alles - jetzt mäht man [nur] noch unten das kleine Stück. Wahrscheinlich eh nicht mehr lange, weil die Murmeltiere inzwischen davon Besitz ergriffen haben. [...] Im Lehnerle. Alle drei Meter ist schon ein Murmeltierloch.' INT: ,Sind die noch mehr geworden?` INTP: ,Mehr sind es wahrscheinlich nicht geworden. Früher hat man natürlich bis ganz oben hin alles gemäht. Und die haben dann immer frisches Futter gehabt. Ja, natürlich sind die auch nicht dumm. Die sind natürlich mit den Mähern ins Tal gegangen. [...] Das frischere Futter haben sie natürlich dort. [...] Speziell, wenn du dort hinüber schaust, zur Mittagszeit, im Barst im Flachen drüben, da sind auf jeden Fall genug!‘

Und das Schlusswort der Einheimischen: Des tuasch aus Tradition weitr und weil mr's uns leischtn kinn.
INT-P: Weil, wenn mr ehrlich sein: Notwendig håt's koan Oanzigr. [...] Es isch hålt, du muasch die Einstellung dazua håbm, rechnen dårfsch nit. [...] Des tuasch aus Tradition weitr und weil mr's uns leischtn kinn. (Abb. 14 und 15)

INT-P: ,Weil, wenn wir ehrlich sind: Notwendig hat es [= die Landwirtschaft] kein Einziger. [...] Es ist halt, du musst die Einstellung dazu haben, rechnen darfst du nicht. [...] Du machst aus Tradition weiter und weil wir es uns leisten können.`

\section{Abkürzungen}

\begin{tabular}{|c|c|}
\hline BEV & $\begin{aligned}= & \text { Bundesamt für Eich- und Ver- } \\
& \text { messungswesen }\end{aligned}$ \\
\hline INT & $=$ Interviewer $/$ in \\
\hline INT-P & $=$ Interviewpartner/in \\
\hline lat. & $=$ lateinisch \\
\hline mhd. & $=$ mittelhochdeutsch \\
\hline OG & $=$ Obergurgl \\
\hline rom. & $=$ romanisch \\
\hline TONK & $\begin{aligned}= & \text { Finsterwalder }(1990 \mathrm{a}) \text { : Tiroler } \\
& \text { Ortsnamenkunde }\end{aligned}$ \\
\hline VE & $=$ Vent \\
\hline WTM & $\begin{aligned}= & \text { Schatz (1955/1956 - 1993): } \\
& \text { Wörterbuch der Tiroler Mun- } \\
& \text { arten }\end{aligned}$ \\
\hline WBÖ & $\begin{aligned}= & \text { Österreichische Akademie der } \\
& \text { Wissenschaften (Hg.) } \quad(1970-\end{aligned}$ \\
\hline
\end{tabular}



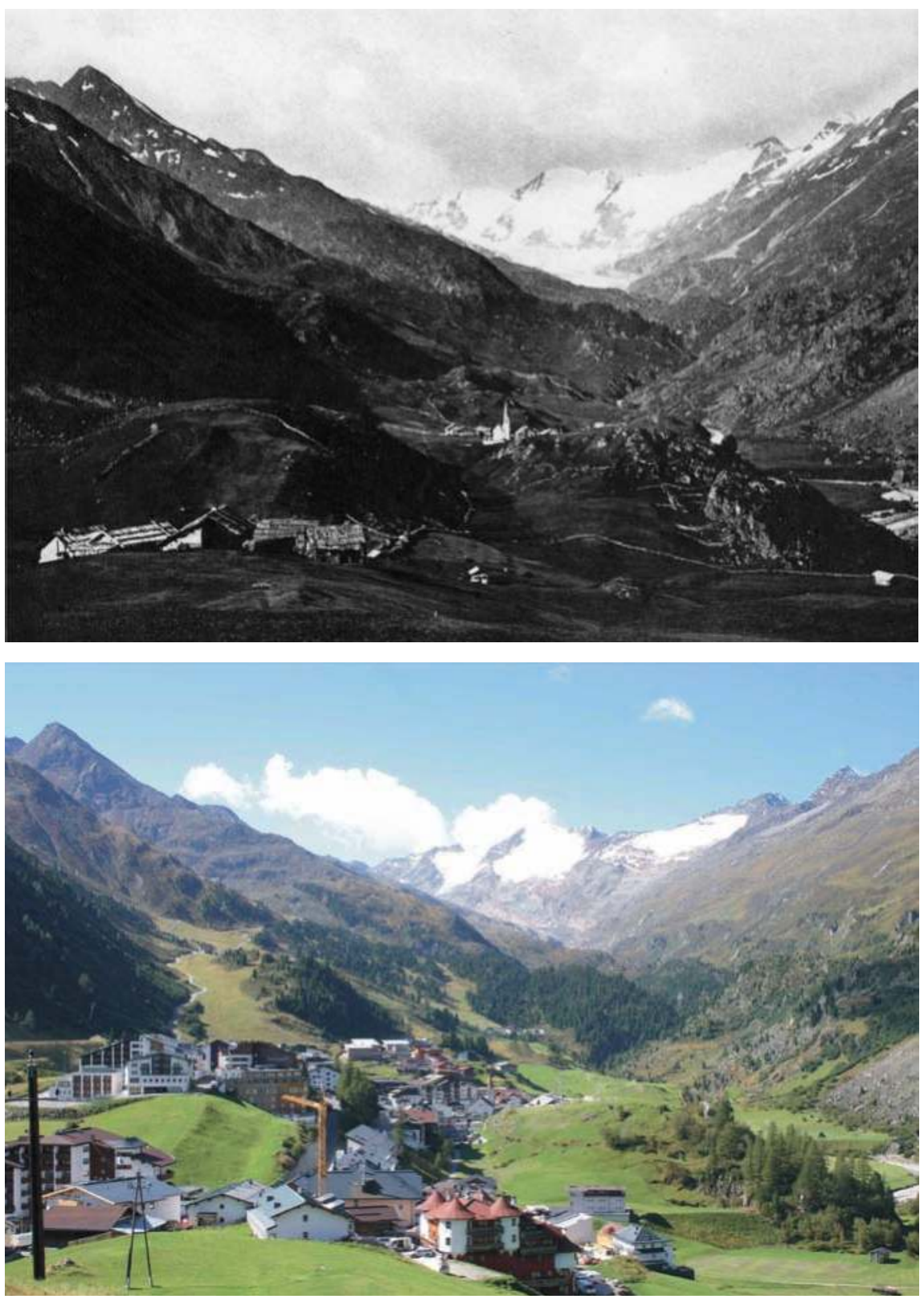

Abb. 14 und 15:

Obergurgl 1880 und 2006: Der Wandel im Ortsbild hat auch einen Wandel der Flurnamen zur Folge. Aber trotz der vielen Gastgewerbebetriebe wird die Landwirtschaft aus Tradition noch weitergeführt. (Foto 1880: B. Johannes, Partenkirchen; Foto 2006: R. Kaufmann) 


\section{Literaturverzeichnis}

Anreiter P. (1997) Zur Methodik der Namendeutung: Mit Beispielen aus dem Tiroler Raum. Innsbrucker Beiträge zur Kulturwissenschaft: Sonderheft 101, Institut für Sprachwissenschaft der Universität Innsbruck, Innsbruck.

Anreiter P., Haslinger M. (2005) Zu den vorrömischen Komponenten des (ost-) alpinen Wortschatzes. In: Indogermanica. Festschrift für Gert Klingenschmitt: Indische, iranische und indogermanische Studien dem verehrten Jubilar dargebracht zu seinem fünfundsechzigsten Geburtstag, Schweiger G. (Hg.), Studien zur Iranistik und Indogermanistik 3, Schweiger VWT, Taimering: 23-43.

Anreiter P., Chapman C., Rampl G. (2009) Die Gemeindenamen Tirols: Herkunft und Bedeutung. Veröffentlichungen des Tiroler Landesarchivs 17, Universitätsverlag Wagner, Innsbruck.

Bätzing W. (1991) Die Alpen: Entstehung und Gefährdung einer europäischen Kulturlandschaft. Beck, München.

Bauer G. (1965) Flurnamengebung als Feldgliederung: Ein kritischer Beitrag zur Methode der Flurnamenstatistik. Mit vier Karten. In: Namenforschung: Festschrift für Adolf Bach zum 75. Geburtstag am 31. Januar 1965, Schützeichel R., Zender M. (Hg.), Universitätsverlag Carl Winter, Heidelberg: 245-263.
Finsterwalder K. (1975) Diphthongierung und Akzentuierung in den Örtlichkeitsnamen in Tirol. In: Tirol-Atlas G: Begleittexte II, im Auftrag des Landes Tirol, Leidlmair L. (Hg.), Universitätsverlag Wagner, Innsbruck: 3-15.

Finsterwalder K. (1990a) [= TONK] Tiroler Ortsnamenkunde: Gesammelte Aufsätze und Arbeiten. Band 2: Einzelne Landesteile betreffende Arbeiten: Inntal und Zillertal, Ölberg H. M., Grass N. (Hg.), Universitätsverlag Wagner, Innsbruck.

Finsterwalder K. (1990 b) Tiroler Familiennamenkunde: Sprach- und Kulturgeschichte von Personen-, Familien- und Hofnamen. Universitätsverlag Wagner, Innsbruck.

Fleischer W. (1962) Zur Frage der Namenfelder. Wissenschaftliche Zeitschrift der Universität Leipzig. Gesellschafts- und sprachwissenschaftliche Reihe H. 2, 11: 319-326.

Haslinger M. (2009) Die Namen von Pfunds. Innsbrucker Beiträge zur Onomastik 6, Praesens, Wien.

Hellfritzsch V. (1990/2010) Zum Begriff „Namenlandschaft" am Beispiel vogtländischer Familiennamen. In: Volkmar Hellfritzsch: (Ostmittel-)Deutsche Namenkunde, Brendler A., Brendler S. (Hg.), baar, Hamburg: 21-31.

Hofinger W. (Hg.) (1991) Tagebuch der Kurazie und Gemeinde in Gurgl: Angefangen im Jahre 1858 von Adolf Trientl. Abschrift einer Kopie der durch Pfarrer 
Franz Danler (1920-1953) ergänzten Handschrift, unveröffentlichtes Manuskript, Innsbruck.

Kat. 35/18-III, Theresianischer Steuerkataster des Gerichtes Petersberg [1776/1777], Tiroler Landesarchiv.

Kaufmann R., Ortner L. (2011) Namenökologie. Eine neue Forschungsrichtung mit mikrotoponomastischen und landschaftsökologischen Methoden: Am Beispiel der Hochgebirgsorte Obergurgl und Vent (Tirol). In: Methoden der Namenforschung. Methodologie, Methodik und Praxis, Ziegler A., WindbergerHeidenkummer E. (Hg.), Akademie Verlag, Berlin: 199-224.

Lynch K. (1968) Das Bild der Stadt. Bauwelt Fundamente 16, Bertelsmann, Berlin München.

Meixner W., Siegl G. (2010) Historisches zum Thema Gletscher, Gletschervorfeld und Obergurgl. In: Glaziale und periglaziale Lebensräume im Raum Obergurgl, Koch E.-M., Erschbamer B. (Hg.), Alpine Forschungsstelle Obergurgl 1, innsbruck university press, Innsbruck: 13-29.

Ortner L. (2009) Flurnamen, Hofnamen, Familiennamen und Gaststättennamen in Obergurgl (Ötztal). In: Kulturraum Tirol: Literatur - Sprache - Medien. Jubiläumsband „150 Jahre Germanistik in Innsbruck", Klettenhammer S. (Hg.), Innsbrucker Beiträge zur Kulturwissenschaft: Germanistische Reihe 75, innsbruck university press, Innsbruck: 461-488.
Österreichische Akademie der Wissenschaften (Hg.) (1970-2004) Wörterbuch der bairischen Mundarten in Österreich (= WBÖ). Bayerisch-Österreichisches Wörterbuch I: Österreich, Wien.

Plangg G. (1998/2011) Zu den Begriffen „Stein"/"Fels" in Tiroler Bergnamen. In: Guntram Plangg: Namenkundliche Schriften zum Raum Tirol, Obererlacher E., Rampl G. (Hg.), Innsbrucker Beiträge zur Onomastik 9, Praesens, Wien: 121-130.

Rampl G. (2011) Zur Entwicklung einiger Alpenwörter im Raum Tirol. In: Die Alpen im Wandel zwischen Risiken und Chancen, Scaramellini G., Dal Borgo A.G. (Hg.), alpine space: man \& environment 12 , innsbruck university press, Innsbruck: $129-140$.

Scharr, K. (2001) Leben an der Grenze der Dauersiedlung: Grund und Boden im „Ötztaler Gebirgsraum“ (Ötztal Schnals - Passeier) vom 13. bis zur Mitte des 19. Jahrhunderts. Schlern-Schriften 314, Universitätsverlag Wagner, Innsbruck.

Schatz J. (1955/1956 - Nachdruck 1993) [= WTM] Wörterbuch der Tiroler Mundarten. Für den Druck vorbereitet von Finsterwalder K., Schlern-Schriften 119, Universitätsverlag Wagner, Innsbruck.

Schmeller J. A. (1877) Bayerisches Wörterbuch. Bd. 2., 2., mit des Verf. Nachträgen verm. Ausg., bearbeitet von Frommann G.K., München.

Schorta A. (1964) Rätisches Namenbuch. Band 2: Etymologien, begründet von 
R. von Planta, bearbeitet und herausgegeben von Schorta A. (Hg.), Francke, Bern.

Stolz O. (1930) Die Schwaighöfe in Tirol: Ein Beitrag zur Siedlungs- und Wirtschaftsgeschichte der Hochalpentäler. Wissenschaftliche Veröffentlichungen des D. u. Oe. Alpenvereins 5, Verlag des Deutschen und Österreichischen Alpenvereins, Innsbruck

Stolz O. (1932) Beiträge zur Geschichte der alpinen Schwaighöfe. Vierteljahrschrift für Sozial- und Wirtschaftsgeschichte 25: 141-157.

Stolz O. (1939) Geschichtskunde von Vent und Rofen. In: Das Venter Tal. Festschrift zum 40-jährigen Bestehen des Deutschen Alpenvereins, Zweig Mark Brandenburg, Deutscher Alpenverein, Zweig Mark Brandenburg, unter Leitung von R. von Klebelsberg zu Thumburg und Mitarbeit von Ekhart E. (Hg.), Bruckmann, München: 74-96.

Stolz O. (1953) Zur älteren Siedlungs- und Herrschaftsgeschichte des Ötztales. Tiroler Heimat. Jahrbuch für Geschichte und Volkskunde 17: 17-24.

Stolz O. (1963) Zur Geschichtskunde des Ötztales. In: Ötztaler Buch, Klebelsberg R. (Hg.), Schlern-Schriften 229, Universitätsverlag Wagner, Innsbruck: 183-247.
Waser E. (2004) Flurnamen. In: Namenarten und ihre Erforschung: Ein Lehrbuch für das Studium der Onomastik. Anlässlich des 70. Geburtstages von Karlheinz Hengst, Brendler A., Brendler S. (Hg.), Lehr- und Handbücher zur Onomastik 1, baar, Hamburg: 349-380.

Windberger-Heidenkummer E. (2001) Mikrotoponyme im sozialen und kommunikativen Kontext: Flurnamen im Gerichtsbezirk Neumarkt in der Steiermark. Europäischer Verlag der Wissenschaften 30, Peter Lang, Frankfurt a.M. u.a.

Die Angaben zu mittelhochdeutschen und althochdeutschen Wortformen wurden den folgenden Werken entnommen und werden der Übersichtlichkeit halber in den Ausführungen nicht näher zitiert:

Lexer M. (1872-1878) Mittelhochdeutsches Handwörterbuch. Zugleich als Supplement und alphabetischer Index zum Mittelhochdeutschen Wörterbuche von Benecke - Müller - Zarncke. 3 Bde., Leipzig.

Köbler G. (1993) Wörterbuch des althochdeutschen Sprachschatzes. Paderborn - Wien (Online-Version: http://www. koeblergerhard.de/ahdwbhin.html). 


\section{Verzeichnis der AutorInnen}

\section{Lorelies Ortner}

Institut für Germanistik

Universität Innsbruck

Innrain 52, 6020 Innsbruck

Lorelies.Ortner@uibk.ac.at

Rüdiger Kaufmann

Institut für Ökologie

Universität Innsbruck

Technikerstr. 25, 6020 Innsbruck

Ruediger.Kaufmann@uibk.ac.at
Yvonne Kathrein

Institut für Germanistik

Universität Innsbruck

Innrain 52, 6020 Innsbruck

Yvonne.Kathrein@uibk.ac.at

Johanna Pidner

Institut für Germanistik

Universität Innsbruck

Innrain 52, 6020 Innsbruck

Johanna.Pidner@student.uibk.ac.at 


\title{
Kapitel 3 | Zum archäologischen Fundbild in Obergurgl
}

\author{
Alexander Zanesco
}

\section{Zusammenfassung}

Für den Raum Obergurgl ist die Anwesenheit des Menschen, wie auch in benachbarten Tälern, innerhalb des frühen Mesolithikums ab der ersten Hälfte des 10. Jahrtausends BPcal nachgewiesen. Den Schwerpunkt der menschlichen Aktivitäten bildete dabei die Jagd. Diese Nutzungsform scheint sich durch das gesamte Neolithikum bis in die Bronzezeit hinein zu erhalten, während parallel dazu im Alpenvorland und in den großen Alpentälern die Landwirtschaft zunehmend Fuß fasst. Spätestens mit dem Beginn der Kupferzeit, um 6300 BP$\mathrm{cal}$, ist eine Nutzung und anthropogene Ausdehnung der Hochweiden zu erkennen. Eine weitere Intensivierung dieser Wirtschaftsform setzt laut Pollenprofilen mit der Bronzezeit ein, wovon auch eine größere Anzahl von Pferchanlagen und Schäferhütten zeugt, die zum Teil mit Radiokarbondaten ab der mittleren Bronzezeit in Verbindung gebracht werden. Eine durchgehende landwirtschaftliche Nutzung bis in unsere Tage scheint schließlich durch weitere Funde aus der älteren und jüngeren Eisenzeit sowie der Römerzeit bis hin zu den ersten Nennungen in Schriftquellen des 13. Jh. n. Chr. gegeben. Die archäologische Forschung muss jedoch weiter intensiviert werden, um zu einem besser fundierten Bild der prähistorischen Siedlungsmuster in der Region zu gelangen.

\section{Abstract}

Human presence in the area of Obergurgl and surrounding valleys is proved for the early Mesolithic from the first half of the $10^{\text {th }}$ millennium BPcal onward. By this time, the main focus of human activity was hunting. This kind of land use seems to continue throughout the Neolithic until the Bronze Age, while in the flatlands and greater alpine valleys agriculture is more and more established. With the beginning of Copper Age, around $6300 \mathrm{BPcal}$, we can recognize the use of alpine grasslands as pastures. By means of pollen diagrams, a further intensification of this kind of economy starts with the Bronze Age. This is also proved by a number of sheepcotes and shepherd huts, which are partly connected with radiocarbon dates starting in the middle Bronze Age. A continuous agricultural use until nowadays seems to be approved by findings from the Iron Ages and Roman times up to the first written records in the middle of the 13th century AD. But for establishing a better founded picture of prehistoric human settlement patterns in the 
region, archaeological investigation needs to be further intensified.

\section{Einleitung}

Parallel zum Rückzug der Gletscher in den Alpen, der etwa 19.000 Jahre vor heute (BPcal - calibrated years before the present) begann, drangen schubweise immer mehr Jäger- und Sammlergruppen in das Alpeninnere vor. Am Beginn dieser Rückzugsphase lag das nördliche Ufer der Adria noch um mehrere hundert Kilometer weiter südlich als heute, weil der Meeresspiegel durch die Bindung von Wasser in den kontinentalen und polaren Eismassen um etwa $120 \mathrm{~m}$ tiefer lag. Erst mit einer verstärkten Erwärmung vor ca. 15.000 Jahren während des BöllingInterstadials stieg der Meeresspiegel rasch an und erreichte innerhalb von etwa 5000 Jahren nahezu heutige Werte (Shackleton et al. 1984). Die Küstenlinien haben sich in diesem Abschnitt wahrscheinlich mit einer Geschwindigkeit verändert, die innerhalb einzelner Generationen deutlich wahrnehmbar gewesen ist. Ähnliches ist auch für das Abschmelzen der Gletscher anzunehmen. Um ca. 16.000 BPcal beginnt im Gegenzug das (neuerliche) Eindringen des Menschen in den alpinen
Raum (Broglio 1994). Eine solche, relativ rasante Umwandlung des Lebensraumes machte entsprechende kulturelle Anpassungen notwendig, die im archäologischen Befund zum Teil erkennbar sind. Ähnliches gilt für den Übergang zum Holozän (Nacheiszeit, ab 11.700 BPcal, Rachoud-Schneider et al. 2002), als mit einem vergleichbaren klimatischen Wandel tiefgreifende Veränderungen im archäologischen Bestand zu bemerken sind. Und auch später reagiert der Mensch auf naturräumliche Veränderungen in unterschiedlicher Weise (Oeggl et al. 2009b), durch eine Mischung aus kultureller Anpassung und Manipulation der Umwelt. Der Zusammenhang von Kultur, Klima und Landschaft ist äußerst komplex, seine Erforschung hat auch Relevanz für künftige Strategien. Wie dieses Wechselspiel im Detail funktionierte, ist Gegenstand einer interdisziplinär arbeitenden (archäologischen) Forschung, die sich nicht nur mit den letztlich marginalen Hinterlassenschaften des Menschen selbst beschäftigt, sondern auch mit den sich verändernden Umweltbedingungen sowie den damit verbundenen Verschiebungen in der Ressourcenverteilung bzw. -nutzung. Archäologische Grundlagenforschung im Hochgebirge wird in Nordtirol erst seit etwa zwanzig Jahren intensiver betrieben ${ }^{1}$. Zuvor wurde prähistorische Landschaftsnutzung, auch für den Raum Obergurgl,

1 Die Auffindung der Mumie vom Tisenjoch („der Mann aus dem Eis“) hatte hier einen ganz wesentlichen Anteil. 
v. a. von archäobotanischer Seite angesprochen (Wahlmüller 1985, Vorren et al. 1993).

$\mathrm{Zu}$ einem wachsenden Verständnis steuern insbesondere die Naturwissenschaften bei. Gezielte archäologische Forschungen im Hochgebirge sollten dabei den Konnex zwischen Natur- und Kulturwissenschaften herstellen und den Menschen im Mittelpunkt sehen. Vieles lässt sich auch, unter geeigneten Voraussetzungen, über das Studium und den Vergleich moderner und submoderner Völker erarbeiten. Daraus lassen sich wiederum Modelle ableiten, welche die Archäologie gezielt überprüfen kann - im prähistorischen wie modernen Kontext.

Das Interesse an Hochgebirgsarchäologie hat sich in den letzten Jahren sehr verstärkt, wovon eine rasch zunehmende Zahl an Publikationen zeugt (z. B. Mandl 2003, Mandl et. al. 2010, Forschungsberichte der ANISA, Mitteilungen der ANISA, Reitmaier 2010, Schäfer 2011). Der Raum Obergurgl bietet dabei für die archäologische Forschung besonders gute Voraussetzungen. ForscherInnen anderer Disziplinen haben seit Jahrzehnten den Boden bereitet, auf dem sich Modelle der prähistorischen Landschaftsnutzung und -formung entwickeln lassen. Es ist zu hoffen, dass diese Forschungen in den nächsten Jahren fortgeführt und intensiviert werden können.
Rekolonisierung der Alpen nach der letzten Eiszeit

Durch das Abschmelzen der Gletscher entstand neuer Lebensraum (Jochim et al. 1999, Boschian 2005). Entsprechend dem Gang der Entgletscherung erfolgte die Rekolonisierung durch Pflanze, Tier und Mensch in horizontaler wie vertikaler (von oben) Richtung, wenn Residualräume bzw. Siedlungen nicht in allzu großer Entfernung lagen. Das teilweise rasche Vordringen des Menschen in die vom Eis frei werdenden Alpen spiegelt sich in der räumlichen und zeitlichen Verteilung von Fundstellen wider (Dalmeri et al. 1998, Dalmeri et al. 2000, Burga 2001, Oberrauch et al. 2010). Waren zunächst nur einzelne, gelegentlich aufgesuchte Örtlichkeiten an den Alpenrändern bekannt (z. B. Riparo Tagliente in den Monti Lessini ab der Ältesten Dryas), beschleunigte sich die Inbesitznahme dieser Landschaft mit dem letzten Interstadial (Bölling/Ältere Dryas/Alleröd bzw. spätes Epigravettién/Magdalenién IV-VI). Ab dem Mesolithikum (Mittelsteinzeit) sind hunderte Fundorte bekannt.

Die überlieferten Tierknochenensembles zeigen zunächst eine gewisse Spezialisierung auf bestimmte Wildarten (bes. Steinbock) und damit ein gezieltes Vordringen in die Gebirgsgegenden (Phoca-Cosmetaton 2006). Unter diesem Blickwinkel müssen die bevorzugten Ressourcen, die sich unter dem Einfluss des Klimawan- 
dels in Richtung Alpenhauptkamm verschoben, ein wesentlicher Faktor für das Eindringen in das Gebirge sowie für veränderte Jagd-, Siedlungs- und Mobilitätsmuster gewesen sein.

Die in Tallagen befindlichen Stationen (z. B. Riparo Soman im Etschtal) zeigen gleichzeitig eine Zunahme von Waldwild in den Schlachtabfällen (z. B. Rothirsch, Wildschwein, Reh, Gämse, Elch u. a., Dalmeri et al. 1998). Andere Tierarten hingegen sind vor ca. 15.000 Jahren aus der Gegend vollständig abgewandert (z. B. Ren, Pferd, Wisent) oder ausgestorben (z. B. Wollnashorn, Schweizer 2002).

Schon im Alleröd (ca. 13.900-12.900 BPcal) errichteten Gruppen ihre Sommerlager (Sekundärstationen) auf Höhen von über 1000-1550 m Seehöhe (z. B. Riparo Dalmeri, 1550 m, Halbhöhle bei Unken/ Salzburg, ca. 650 m, Dalmeri et al. 1998). In der etwas kühleren Jüngeren Dryas (ca. 12.900-11.700 BPcal) stießen die Gletscher wieder vor und überfuhren zum Teil den neuen Waldbestand. Der Raum Obergurgl war zu dieser Zeit noch hunderte Meter hoch von Eis bedeckt ${ }^{2}$.

Mit dem Holozän ( $\mathrm{ab} 11.700$ BPcal) beginnt auch das archäologische Mesolithikum. Jäger- und Sammlergruppen durchstreifen bald nahezu den gesamten alpinen Raum. Ihr Leben war allem Anschein nach von einer durch jahres- zeitlich-klimatischen Wechsel bedingten Mobilität zwischen Hochgebirge und Tal geprägt. Dabei ist die Zweiteilung in Sommer- und Winterlager vermutlich zu kurz gegriffen. Besonders der Herbst muss eine wichtige Jagdsaison gewesen sein, weil sich das Wild in dieser Zeit durch Fettansatz und dichteres Fell auf die Winterzeit vorbereitet. Diese Qualitäten müssen für seine Nutzbarkeit von entscheidender Bedeutung gewesen sein (Watanabe 1973, Phoca-Cosmetaton 2006). Es ist z. B. denkbar, dass manches Jägerlager in mittlerer Lage vor allem im Herbst genutzt wurde.

Die Waldgrenze erreichte im Raum Obergurgl bereits vor ca. 11.000 Jahren eine Meereshöhe um 2300 m (Bortenschlager 2010). Die Böden großer Alpentäler waren dagegen von Systemen aus Seen, Teichen und Sumpfgebieten geprägt (Dalmeri et al. 1998, Patzelt 2008). Dementsprechend hat hier neben der Jagd und dem Sammeln pflanzlicher Ressourcen auch die Nutzung von Fischen und Mollusken eine wichtige Rolle gespielt (Bazzanella 2001, Bazzanella et al. 2007).

Im inneren Ötztal ebenso wie auch vermutlich am gesamten Alpenhauptkamm setzt die Begehung durch den Menschen erst mit dem Mesolithikum ein. Hier dauert die „aneignende“ Wirtschaftsform (Foraging) dann aber auch wesentlich län-

2 Im Fotschertal (Ullafelsen) ist beispielweise auf $1869 \mathrm{~m}$ Seehöhe für das Bölling-Alleröd-Interstadial die Ausbildung eines biogenen Bodens nachgewiesen, der in der Jüngeren Dryas von äolischen Ablagerungen bedeckt wurde. Erst oberhalb dieses Horizonts sind Artefakte und damit die Anwesenheit des Menschen nachgewiesen (Geitner et al. 2010). 
ger an als im Alpenvorland. Die Abgrenzung zu, oder vielmehr das Nebeneinander mit „produktiven“ Subsistenzweisen (Ackerbau, Viehzucht etc.) in Tallagen sollte hier daher besonders gut zu erforschen sein.

Im jüngsten Abschnitt des Mesolithikums (ab ca. 9000 BPcal) und im frühen Neolithikum scheint die Begehung des inneralpinen Raumes etwas zurückzugehen, gleichzeitig zeichnet sich in den Voralpen ein Vordringen der Siedeltätigkeit in größere Höhen ab (Dalmeri et al. 1998). Ein Zusammenhang mit einer klimatisch ungünstigeren Phase (Rotmoosschwankung I, 6600-6200 BPcal) wäre denkbar (Oeggl et al. 2009b, Joerin et al. 2008, Nicolussi 2009).

Klima und Ressourcen, Siedlung und Mobilität

Das Holozän beginnt mit einer ausgesprochenen Warmphase, die mit Unterbrechungen (Joerin et al. 2008, Oeggl et al. 2009b, Bortenschlager 2010) bis vor etwa 6600 Jahren anhielt (Präboreal-Boreal-frühes Atlantikum). Dabei erklomm die Waldgrenze vorübergehend größere Höhen als das heute möglich wäre. Auch die Gletscher haben sich zeitweise weiter zurückgezogen (Nicolussi 2009). Mit der Ansiedlung neuer Vegetation fand sich auch die Tierwelt ein. Neben verschiedenem Stein-, Rot- und Niederwild kann man auch für unseren Raum Räuber wie Bär, Luchs, Wolf und verschiedene Greifvögel annehmen (Schweizer 2002) ${ }^{3}$. Besonders in den Tallagen spielten auch Kleintiere, Fische, Schildkröten, Vögel (Vogeleier), Weichtiere sowie vielleicht auch Insekten (Morris 2008) und Honig eine zunehmende Rolle bei der Nahrungsversorgung. Ein breites Nahrungsspektrum, Mobilität und stärkere soziale Vernetzung (gruppenübergreifende linages, abgeschwächte Territorialität) reduzieren das Risiko von Mangelperioden und den Aufwand für die Nahrungsbeschaffung. Tiere lieferten aber nicht nur Nahrung in Form von Fleisch, Fett, Blut, Eiern und Honig, sondern auch Felle, Knochen, Geweihe, Sehnen, Federn und andere Rohstoffe für den täglichen Bedarf. Die Berge boten daneben auch Kräuter, essbare Wurzeln, Wildfrüchte, Nüsse, Hölzer, Harze, Fasern und Baste. Pflanzliche Nahrung wird eine ebenso große Rolle gespielt haben wie tierische.

Wenn die Ressourcen einer Region nicht ausreichen, um den Jahresbedarf zu decken, wird Wanderschaft zur Notwendigkeit. Der zeitweise Überfluss einer Ressource nützt nur vorübergehend, wenn sie nicht gelagert werden kann (Testart

3 Für Nordtirol stehen archäozoologische Untersuchungen zum Mesolithikum vor allem erhaltungsbedingt noch aus. 
1982). Je besser und vorhersagbarer an einem Ort die Versorgung ist, desto weniger besteht umgekehrt die Notwendigkeit zu einer umherziehenden Lebensweise. Im unteren Etschtal wurde an den Gewässern beispielsweise gefischt. Dies stellte eine verhältnismäßig sichere Lebensgrundlage (Bazzanella et al. 2007) dar. Ein positiver Aspekt zyklischer Wanderung ist aber auch die Schonung des örtlichen Bestan- des. Offenbar überwogen die Vorteile regelmäßiger Ortswechsel. Der Gang ins Gebirge „lohnte“, wenn der Nutzen den Aufwand übertraf (Madsen et al. 2000). Dass dem so war, zeigen die Fundstellen. Es waren offenbar genügend Anreize vorhanden, sich auch in den Zentralalpen nach jagdbarem Wild, nutzbaren Pflanzen und "gesellschaftlichen Nutzbarkeiten “4 umzusehen. Wanderschaft ist nicht ein-

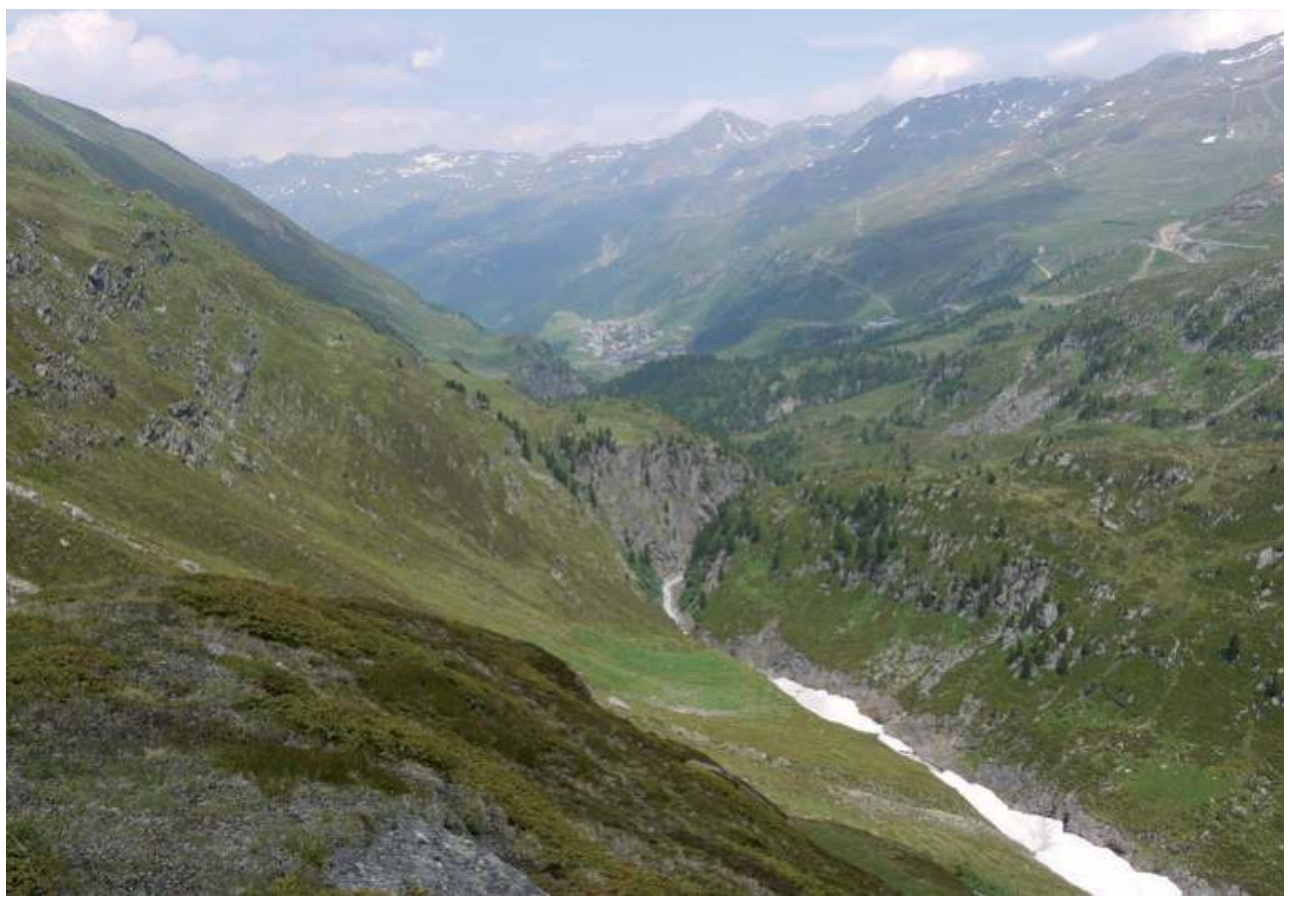

Abb. 1:

Blick über den Beilstein (Bildmitte) nach Obergurgl, Blickrichtung NO (Foto: A. Zanesco)

4 Auch „moderne“ Jäger- und Sammlervölker der mittleren Breiten bestreiten fast regelhaft einen größeren Teil ihres Lebensunterhaltes aus dem Sammeln von Pflanzenteilen und kleinen Tieren, und dennoch besitzt die Jagd einen besonderen gesellschaftlichen Stellenwert. Sie ist meist eine Domäne des Mannes und ein wichtiger Teil des Selbstverständnisses des betreffenden Volkes. Daher ist die Frage nach dem Warum nicht nur über eine mathematische Kosten-Nutzen-Rechnung zu beantworten. Auch andere ideelle Werte wie der Stellenwert der Landschaft (Berge) in der geistigen Vorstellungswelt wären zu berücksichtigen. 
fach Tradition, sondern Notwendigkeit. Die Spuren folgen dabei einer Logik, die mit der Landschaftsgestalt und den vorfindlichen Ressourcen in Zusammenhang steht (Kompatscher et al. 2007). Hatte man einmal Höhen über der Waldgrenze erreicht, konnte man sich vielfach in weitgehend flachem und offenem Gelände rascher fortbewegen. Dort wo Wald und offenes Gelände aufeinander treffen, ist auch die Artenvielfalt besonders groß (Waldgrenzökoton). Dementsprechend finden sich die Hinterlassenschaften die- ser Menschen vor allem an Idealrouten nahe der Waldgrenze zu und zwischen bevorzugten Jagd- und Sammelgebieten. Die häufigste Herkunft des Rohmaterials der Artefakte in Vent spricht beispielsweise für eine Erschließung des inneren Ötztales aus Richtung Süden (Leitner 1999) ${ }^{5}$. Vermutlich hängt das mit den günstigeren klimatischen Bedingungen südlich des Alpenhauptkamms zusammen, aber auch mit dem dort anstehenden, qualitätvollen Silex (z. B. im Nonstal, Monte Baldo, Lessinische Alpen). Nicht zuletzt gaben

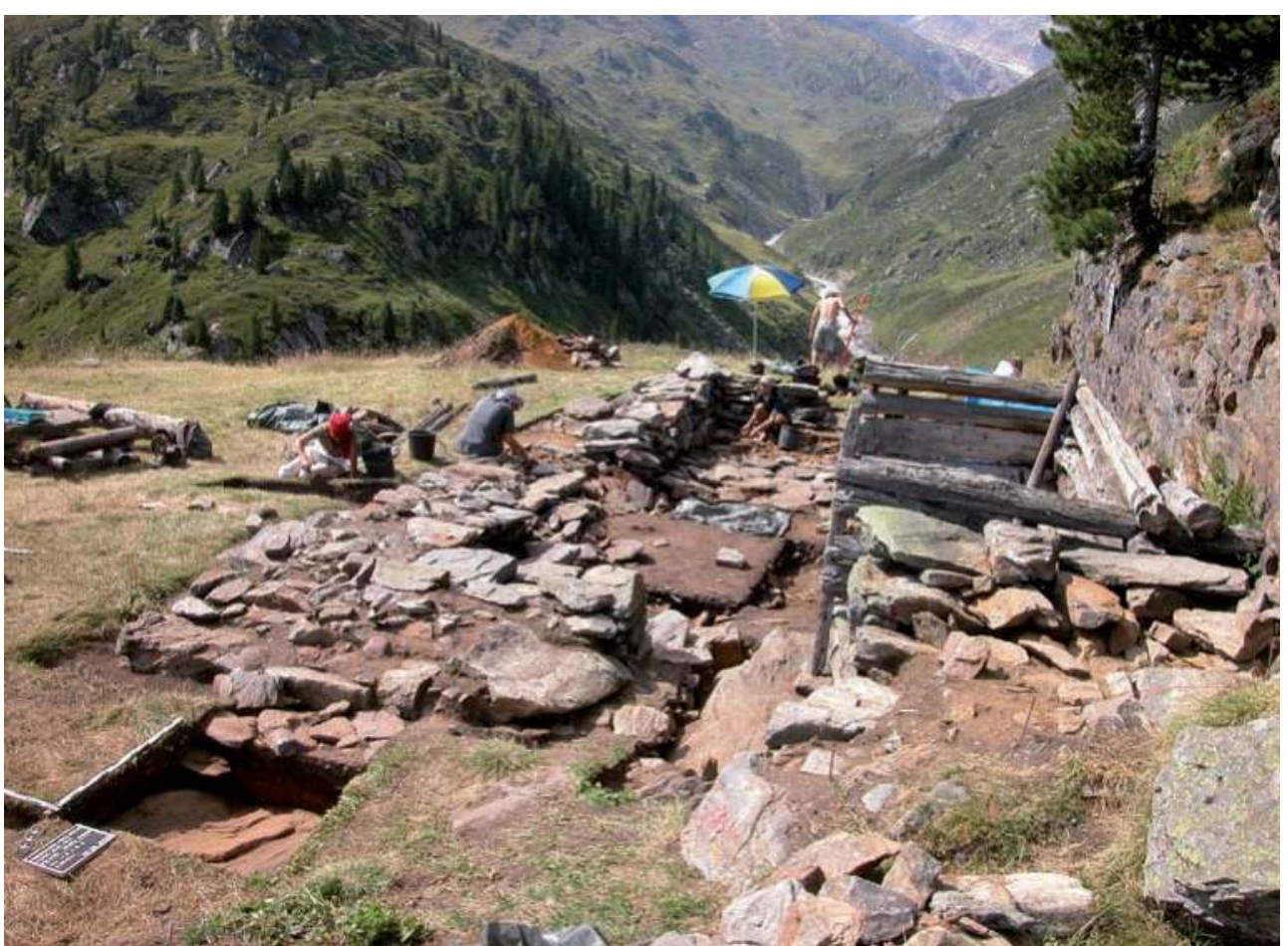

Abb. 2:

Ausgrabungen bei der Almwüstung Beilstein, Blickrichtung S (Foto: A. Zanesco)

5 Herkunftsbestimmungen des am Beilstein geborgenen Silexmaterials sind noch ausständig. 


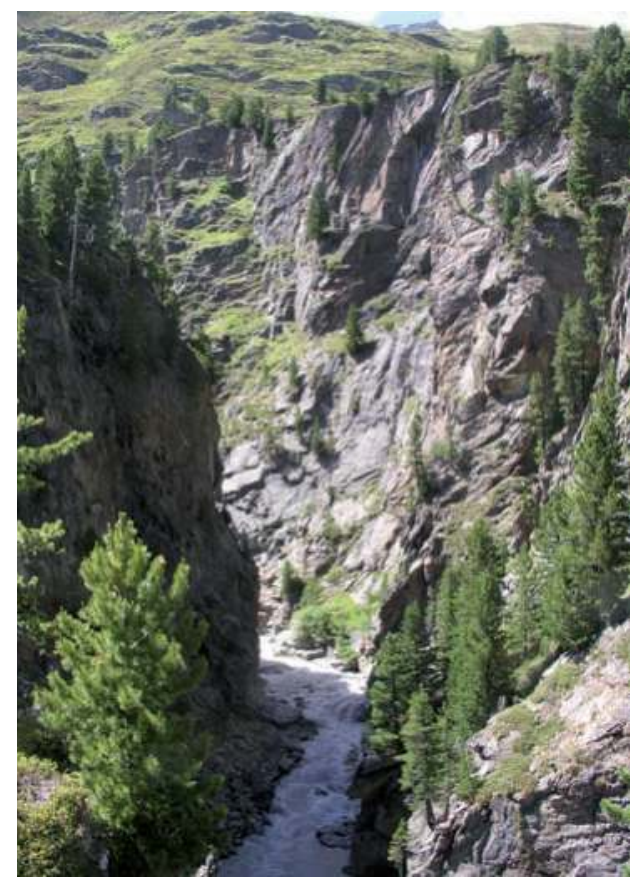

Abb. 3:

Schlucht des Gurgler Baches beim Beilstein (oben an der Felskante rechts, ca. $100 \mathrm{~m}$ über dem Talboden), Blickrichtung W (Foto: A. Zanesco)

diese Materialien selbst einen wichtigen Antrieb zur Wanderschaft (Crotti 2005). Mit Ausnahme von Bergkristall finden sie sich vorwiegend in den Kalk- und Juraformationen der Voralpen (Binsteiner 1994). Sie belegen einen Aktionsradius von hunderten Kilometern.

Mesolithische Basislager reihen sich ab etwa 11.300 bzw. 11.200 BPcal in einer gewissen Häufigkeit entlang der Ränder des Etschtales um Trient (ca. 210-250 m üNN). Aber auch im oberen Etsch- und Eisacktal sind mesolithische Siedlungsbefunde bekannt (z. B. Stufels bei Brixen,
Lunz 1986). Im Inntal fehlen bisher die Nachweise, was aber wohl nur eine Forschungslücke ist (Patzelt 2008, Zanesco 2008).

Schon ab dem 8. Jahrtausend v. Chr. zeigen Fundstellen am Alpenhauptkamm die Begehung durch den Menschen an (Margreiter 2009, Oberrauch et al. 2010, Schäfer 2011). Sekundärlager für längerfristige Aufenthalte im Gebirge und temporäre Lagerplätze erreichen bis hin zum Alpenhauptkamm vielfach Höhen um $2300 \mathrm{~m}$. Wichtige Sekundärlager im Raum Obergurgl sind der Beilstein (Abb. 1-4, Zanesco 2007) und die Station im Rofental/Vent (Leitner 1999). Die bisher vorliegenden ${ }^{14} \mathrm{C}$-Daten aus Kulturschichten setzen auch hier im Laufe der ersten Hälfte des 8. Jahrtausends v. Chr. ein. Gleiches gilt für Jagdlager in den angrenzenden Regionen, etwa im Pfelderertal und Hinterpasseier (Moser et al. 1986, Oberrauch et al. 2010).

Die gegenüber der Eiszeit veränderten Jagdgewohnheiten spiegeln sich auch im Fundmaterial wider. Schon seit dem Spätpaläolithikum belegt eine zunehmende Tendenz zur Verkleinerung der Geschoßeinsätze aus Silex neue Jagdwaffen, die aus mehreren Teilen zusammengesetzt für kleineres Wild verwendet wurden. Sie erlaubten auch eine ökonomischere Nutzung des zur Verfügung stehenden Rohmaterials. Die Typologie der Geräte zeigt im Laufe des Mesolithikums eine regional leicht unterschiedliche Entwicklung bei gleichen Grundtendenzen. Es besteht 


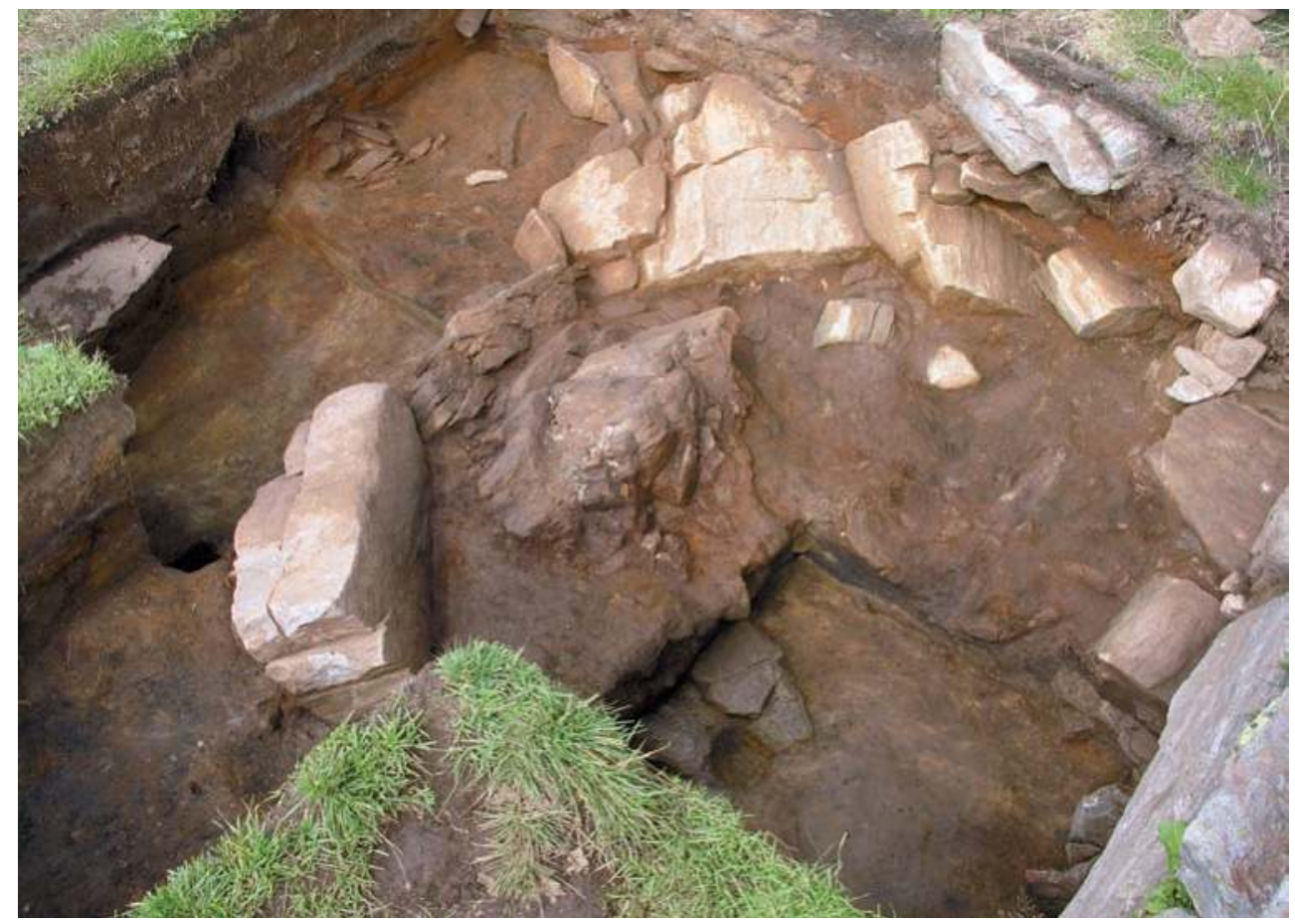

Abb. 4:

Mesolithische Befunde am Beilstein, halbkreisförmige Steinsetzung inmitten von Kulturschichten (Foto: A. Zanesco)

natürlich ein enger Zusammenhang zwischen Jagdtechnik und Ergologie der Jagdgeräte.

Fließender Übergang zu produktiven Wirtschaftsformen

Die sehr mobile Lebensweise dieser Menschen war auch eine wichtige Voraussetzung für Kulturkontakte, Kulturvermittlung und kulturelle Veränderungen. Das dürfte sich besonders am Übergang zum Neolithikum (Jungsteinzeit) bemerkbar gemacht haben. In dieser Periode (ab ca. 7500 BPcal) werden Jäger und Sammler zu Hirten, die uns in der Überlieferung der Gaban-Gruppe im Trentino und in Südtirol (Pedrotti 1998, Pedrotti 2009), vereinzelt auch in Nordtirol (Töchterle 2009) entgegentritt. Dabei ist die Frage nach Akkulturation oder Zuwanderung noch in Diskussion. Wichtig ist in diesem Zusammenhang auch die Frage nach einem zunehmenden Populationsdruck als Triebfeder ökonomischer und kultureller 
Anpassung. In den Abfällen finden sich bereits zu einem nennenswerten Anteil Knochen von Haustieren (z. B. Ziege, Schaf, Rind, Schwein). Auch Getreide und Leguminosen sind nachgewiesen. Keramik war schon in Gebrauch. Doch das Silexinventar ist von dem der unmittelbar vorangegangenen Mesolithiker nur schwer zu unterscheiden. Es gibt auch keinen Grund anzunehmen, dass diese Leute nicht saisonal zur Jagd ins Gebirge gegangen sein sollten. Schließlich belegen die Nordtiroler Funde auch die Überquerung des Alpenhauptkamms. $\mathrm{Ob}$ sie schon ihr Nutzvieh mit in die Berge führten, ist nicht geklärt. Wie das Territorium sollten auch die Haustiere bereits in irgendeiner Form Gemeinschaftsbesitz gewesen sein. Auch dieses war ja sicher nicht nur ein geographisches Gebiet. Sein Inhalt zählte sowie auch die vorhandenen Ressourcen und ideelle Werte.

Anhand von Pollenprofilen im Raum Vent/Obergurgl wird der Beginn der Hochweidenutzung durch aufgetriebenes Vieh für die Zeit ab ca. 6300 BPcal angenommen (Bortenschlager 2000, Bortenschlager 2010). Bereits Brandhorizonte des 6.- 5. Jahrtausends v. Chr. könnten für anthropogene Absenkungen der Waldgrenze sprechen, die man teilweise in Verbindung mit einer Ausdehnung der Weideflächen sehen kann (Gernot Patzelt, persönl. Mitteilung; Patzelt et al. 1997, Bortenschlager 2010, Oberrauch et al. 2010). Allerdings müssten diese Ereignisse erst eindeutig mit menschlichen Ak- tivitäten in Verbindung gebracht werden. Andererseits ist es nicht sehr wahrscheinlich, dass nach dem Beginn der Viehzucht im Etschtal ein Jahrtausend bis zur Bestoßung der Hochweiden vergangen sein sollte, wenn diese Leute ohnedies zur Jagd ins Gebirge gingen. Eher war der Effekt zunächst noch zu gering, um ihn an den Pollenprofilen erkennen zu können.

Auch die Hirten und Jäger mussten, zur durchgehenden Versorgung ihres Viehs und zur Nutzung der Ressourcen im Gebirge, eine saisonale Wanderwirtschaft praktizieren. Inwieweit und $a b$ wann diese Wanderungen die Form von Transhumanz annahmen, bei welcher ein Teil der Bevölkerung zwischen Sommer- und Winterweiden jeweils das heimische Dorf passierte, ist eine von vielen offenen Fragen (Spindler 2005). Man kann spätestens jetzt aber beständige Talsiedlungen annehmen.

Neolithische Artefakte sind im Hochgebirge bisher erst in einer späteren Phase deutlich zu erkennen, weil sich etwa jene der Gaban-Gruppe nur schwer von älterem Material differenzieren lassen und eine genaue Durchsicht wohl noch aussteht. Verschiedene Pfeilspitzenfunde (Niederwanger 1984, Oberrauch et al. 2010), die typisch für die Vasi a Boca Quadrata-Kultur (VBQ) sind, belegen nicht nur die Anwesenheit ihrer Träger aus dem oberitalienischen und Trentiner Raum, sondern auch deren Interesse an der Jagd. Sie dürften die unmittelbaren Nachkommen der vorangegangenen, 


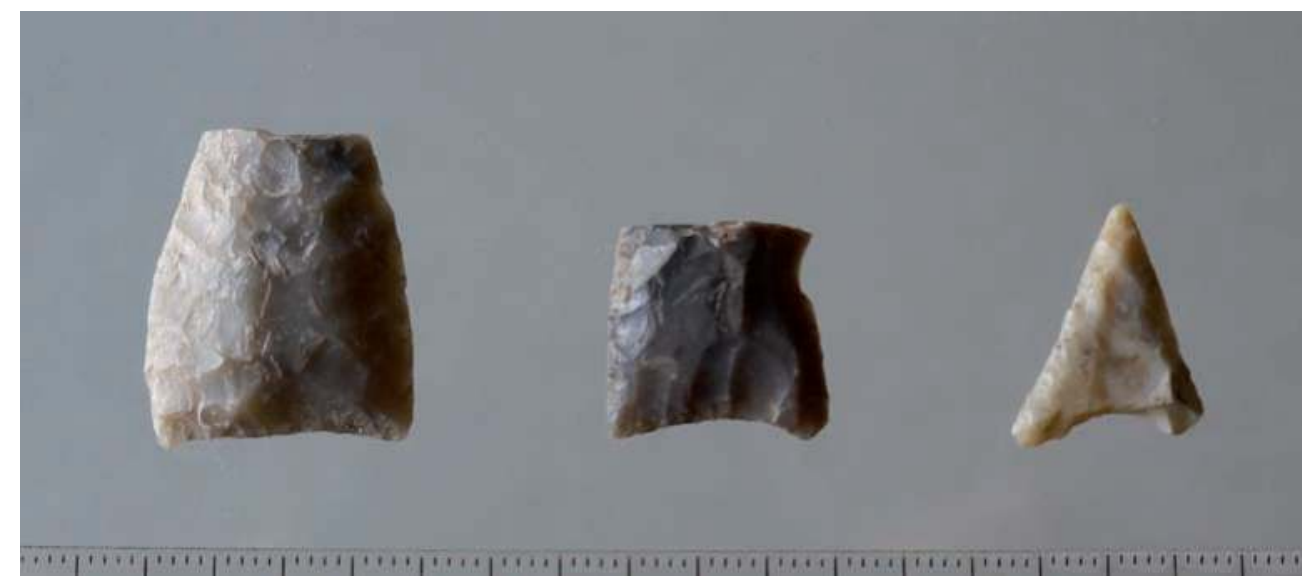

Abb. 5:

Funde vom Beilstein: Neolithische und bronzezeitliche Pfeilspitzen (Foto: A. Zanesco)

frühneolithischen Kulturen gewesen sein, zumal sie teilweise dieselben Siedlungsplätze nutzten. Weitere Projektile stammen aus der Kupferzeit. Auch der Beilstein bei Obergurgl hat derartige Funde erbracht. Die jüngsten Pfeilspitzen (Abb. 5) datieren hier wie in angrenzenden Gebieten noch deutlich später. Damit ist für diese Lokalität zunächst eine Kontinuität als Jagdplatz bis tief in die Bronzezeit hinein abzusehen. Ein differenzierterer Blick wird aber erst mit Abschluss der Fundauswertung möglich sein.

\section{Landwirtschaft und Jagd}

Als Neolithikum wird ein Komplex von materiellen Kulturhinterlassenschaften verstanden, die mit Viehzucht, Ackerbau,
Sesshaftigkeit, Bevorratung und allen damit in Zusammenhang stehenden Merkmalen in Verbindung gebracht werden, wie etwa Keramik, geschliffene Steinartefakte, Hausbau, Siedlungen, Gräberfelder, komplexe Gesellschaften usw. Diese Eigenschaften treten in verschiedenen Regionen in unterschiedlicher Kombination und zeitlicher Abstufung auf. Sie sind auch nicht einfach an „Ackerbauern und Viehzüchter" gebunden. Die Charakterisierung als Neolithikum setzt ein Bündel dieser Kulturäußerungen voraus. In Mitteleuropa, nördlich und südlich der Alpen, beginnt dieser Abschnitt um 7500 BPcal. Eine intensivere landwirtschaftliche Nutzung des Hochgebirges zeigt sich aber erst mit der Bronzezeit. Dabei lässt sich ein sukzessives Eindringen der Landwirtschaft in das Alpeninnere erkennen. Zunächst sind für das 5. Jahrtausend v. Chr. Eingriffe in die 
Wälder der tieferen Zonen zu beobachten (Oeggl 2009, Oeggl et al. 2009b). Klimaxbaumarten gehen zurück, dafür zeigen sich erste Siedlungs- und Kulturzeiger. Im Unterinntal wie im Überetsch herrscht zu dieser Zeit Eichenmischwald vor. Eicheln konnten als zeitweiser Ersatz für kultivierte Pflanzen dienen. Im Alpenvorland werden Gerste, Emmer, Einkorn, Erbse, Lein und Schlafmohn angebaut. Auch Wildobst und Nüsse tragen einen Teil zur Kost bei, daneben Fleisch von Haustieren und zu einem geringeren Anteil von Wild.

Etwa zur selben Zeit, ungefähr 6600 BPcal, hat sich das holozäne Klimaoptimum abgeschwächt (Rotmoosschwankung I; Joerin et al. 2008, Nicolussi 2009). Gleichzeitig sank auch die Baumgrenze bzw. lichteten sich die Wälder im Grenzökoton. Die Sommertemperaturen sanken und erreichten ca. $6400 \mathrm{BPcal}$ ein Minimum unter gleichzeitigem Anstieg der Niederschläge. Auch die Gletscher stießen wieder vor. Es ist auffällig, dass gerade in dieser Periode die Hochweiden, vielleicht durch den Klimadruck bedingt, (verstärkt) erschlossen bzw. durch Rodung nach unten gedrückt wurden (Bortenschlager 2000). Vor etwa 6200 BPcal verbesserten sich die klimatischen Bedingungen wieder. Die Gletscher zogen sich zurück und der Wald gewann an Höhe (Nicolussi 2009). Erst im Endneolithikum, ca. 6000-5000 BPcal, werden Bereiche des inneren Gebirges besiedelt (z. B. Vinschgau, Engadin, Oberinntal).
Hier überwiegen Nadelwälder, besonders die Fichte (Oeggl 2009). Auch in einem Zeitabschnitt nach ca. 5600 BPcal (Rotmoosschwankung II) sind die klimatischen Bedingungen ungünstig (Magny et al. 2006, Joerin et al. 2008, Oeggl et al. 2009b). Diese Periode scheint mit Migrationsbewegungen und der Erschließung neuer Ressourcen im Inneren des Gebirges zusammenzufallen.

Etwa zum gleichen Zeitpunkt dürfte der Bergbau eine zunehmende Rolle gespielt haben. Ab ca. 6300 Jahren BPcal sind in Tallagen (z. B. Brixlegg, Thaur) erste Kupferobjekte nachgewiesen. Montanistische Prospektion erfolgte spätestens ab diesem Zeitpunkt. Für das innere Ötztal sind aber keine Vorkommen bekannt, die einen Abbau ermöglicht hätten. Hier sollten nach wie vor Jagd und Weide die wichtigsten Wirtschaftsfaktoren gewesen sein. Das erst viel später fassbare Bergbaurevier am Schneeberg bleibt bisher ohne Beleg für prähistorische Aktivitäten dieser Art.

Offenbar bestand also ein Jahrtausende langes Nebeneinander von saisonaler Gebirgsjagd und Landwirtschaft in den großen Tälern und im Alpenvorland, bis mit der frühen Bronzezeit, um 4300 BP$\mathrm{cal}$, intensivierte, landwirtschaftliche Aktivitäten auch im Hochgebirge erkennbar werden. Wenige Jahrhunderte danach, etwa 3700-3600 BPcal ist wiederum eine kühlere Phase zu bemerken (LöbbenSchwankung). Die Talsiedlungen rücken langsam näher an die Hochweiden, was u. 
a. die Wege verkürzt. Als weiterer wichtiger Faktor wird der Bergbau spätestens mit der Bronzezeit zumindest indirekten Einfluss ausgeübt haben (z.B. Nahrungsversorgung der im Bergbau Tätigen). Damit wird die Frage nach dem Beginn der Alpwirtschaft wichtig. Die Verarbeitung von Milch zu haltbarem Käse sollte längst üblich gewesen sein (Dammers 2003). Ebenfalls wichtig ist in diesem Zusammenhang die noch ungelöste Frage nach dem Beginn der Heumahd und damit der Überwinterung einer größeren Anzahl von Vieh (Gleirscher 2010). Indirekt hängt daran auch die gleichermaßen ungeklärte Frage nach dem Beginn der Bewässerung auf den bewirtschafteten Flächen.

Wann die ersten Gehöfte den Raum Obergurgl erreichten, ist noch völlig unsicher. Die Schriftquellen setzen erst Mitte des 13. Jh. n. Chr. ein. In den Pollenprofilen lässt sich aber eine kontinuierliche Nutzbarmachung der Flächen erkennen, beginnend mit der frühen Bronzezeit und bis heute andauernd. Ein wesentliches Charakteristikum dieser Aktivitäten ist die Erweiterung der Weideflächen durch Brandrodung von oben her (Patzelt 2000).
Landschaftsbild und Wege in Obergurgl

Das Gurglertal erstreckt sich ab Zwieselstein in NNO-SSW-Richtung zum Alpenhauptkamm und trennt sich hier vom westlich parallel verlaufenden Venterund Niedertal. Es ist von diesem durch eine Bergkette getrennt, die nach Süden ansteigend Höhen um 3550 m erreicht (Ramolkogel). Die steile Talflanke geht auf 2400-2500 m Höhe in eine langgezogene und teilweise breite Talschulter über, welche ideale Hochweideflächen bietet („Seenplatte“, ca. 6 km lang). Die östliche Talseite ist von geringerer Steilheit und wird von mehreren, hoch gelegenen Quertälern durchschnitten. Talbodenreste auf 2200-2300 m Höhe, die sich als Hochweiden eignen, sind hier nur abschnittsweise erhalten (z. B. Angereralm, Äußere und Innere Haide, Böden). Auch die östliche Bergkette erreicht teils Höhen bis $3480 \mathrm{~m}$. Im Süden wird das Tal von Bergen ähnlicher Höhe abgeschlossen. Gletscher sind auf der Ostseite durch Wetterlage und Relief bedingt deutlich stärker ausgeprägt und reichen heute ausnahmsweise bis auf $2400 \mathrm{~m}$ herab, bleiben sonst aber über 2500-2700 m. An der Westseite sind die Gletscherflächen deutlich kleiner und liegen meist oberhalb von $2800 \mathrm{~m}$.

Südlich von Zwieselstein (1470 m) durchschneidet die Gurgler Ache eine Talstufe von etwa 150 m Höhe. Danach steigt der 
Talboden regelmäßig bis zu einer zweiten, kleineren Stufe kurz vor Pill an (1782 $\mathrm{m})$. In diesem Talabschnitt zweigt das etwa $300 \mathrm{~m}$ höher gelegene Timmelstal in Richtung Osten ab und quert über das Timmelsjoch ins Passeirer Timmelstal. Diese Route wird vor allem auf Südtiroler Seite, aber auch am Joch selbst (Schönbodenlacke) durch eine Reihe von Fundstellen belegt, die bis in das frühe Mesolithikum reichen (Niederwanger 2007, Oberrauch et al. 2010, Schäfer 1999: 2, Schäfer 2001). Als niederster Übergang des Alpenhauptkamms zwischen Brennerund Reschenpass war das Timmelsjoch (2509 m) offenbar seit der Mittelsteinzeit von Bedeutung. Der jüngste prähistorische Fund bei der Schönbodenlacke ist eine spätlatènezeitliche Armbrustspiralfibel mit hochgezogenem Fuß, deren Vorläufer in die mittlere Latènezeit reichen (3./2. Jh. v. Chr.). In ähnlicher Form ist sie noch im 1. Jh. n. Chr. in Verwendung (Adam 1996, Zanesco 2007b, Oberrauch et al. 2010).

Im folgenden, mittleren Abschnitt (ca. $4 \mathrm{~km}$ ) wird das Gurglertal relativ eben und breit und erreicht bei Obergurgl eine Meereshöhe um 1900 m. Zwischen Pill und Obergurgl lagen vermutlich die ältesten, von der Landwirtschaft geprägten Siedlungsbereiche. Archäologische Forschungen sind hier jedoch nicht erfolgt. Die rege Bautätigkeit der letzten Jahrzehnte dürfte auch einen Großteil älterer Siedlungsspuren ausgeräumt haben.
Nach weiteren etwa $3,5 \mathrm{~km}$, bei der Einmündung des Langtaler Baches, liegt der Talboden auf $2200 \mathrm{~m}$. Dahinter schließt die Gletscherzone an. Prospektionen, Sondagen und Ausgrabungen beschränken sich bislang auf die Zone oberhalb etwa 2000 m Seehöhe. Die meisten prähistorischen Fundstellen reihen sich entlang der potentiellen Waldgrenze um $2300 \mathrm{~m}$. Innerhalb des Waldgürtels befindet sich der bislang wichtigste Fundort, der Beilstein (Abb. 1-4).

Obergurgl liegt abseits der Transitroute über das Timmelsjoch/Timmelstal, etwas weiter südlich. Das Tal ist nach Norden hin offen und wird sonst durch hoch gelegene Jöcher erreicht. Übergänge führen in das Ventertal (Gurgler Scharte, 2932 m) bzw. Niedertal (Ramoljoch, 3189 m) im Westen, dann weiter über das Niederjoch $(3017 \mathrm{~m})$ ins Schnalstal. Nach Osten führen Pfade durch das Königstal (Königsjoch, $2825 \mathrm{~m}$ ) und das Ferwalltal (Aperes Ferwalljoch, 2902 m) ins Seewertal/Hinterpasseier und weiter über das Rauhe Joch (2926 m) ins Pfelderertal, das auch über das Rotmoostal (Rotmoosjoch, $3055 \mathrm{~m}$ ) und Langtal (Langtaler Joch, $3031 \mathrm{~m}$ ) erreicht wird. Schließlich gelangt man über das Gurgler Eisjoch (3151 m) nach Südosten in das Pfossental und von dort weiter über das Eisjöchl (2900 $\mathrm{m})$ wiederum ins Pfelderertal/Passeiertal bzw. nach Westen ins Schnalstal. Einzig das Gaisbergtal besitzt keinen geeigneten Übergang am Talschluss. Diese Täler er- 


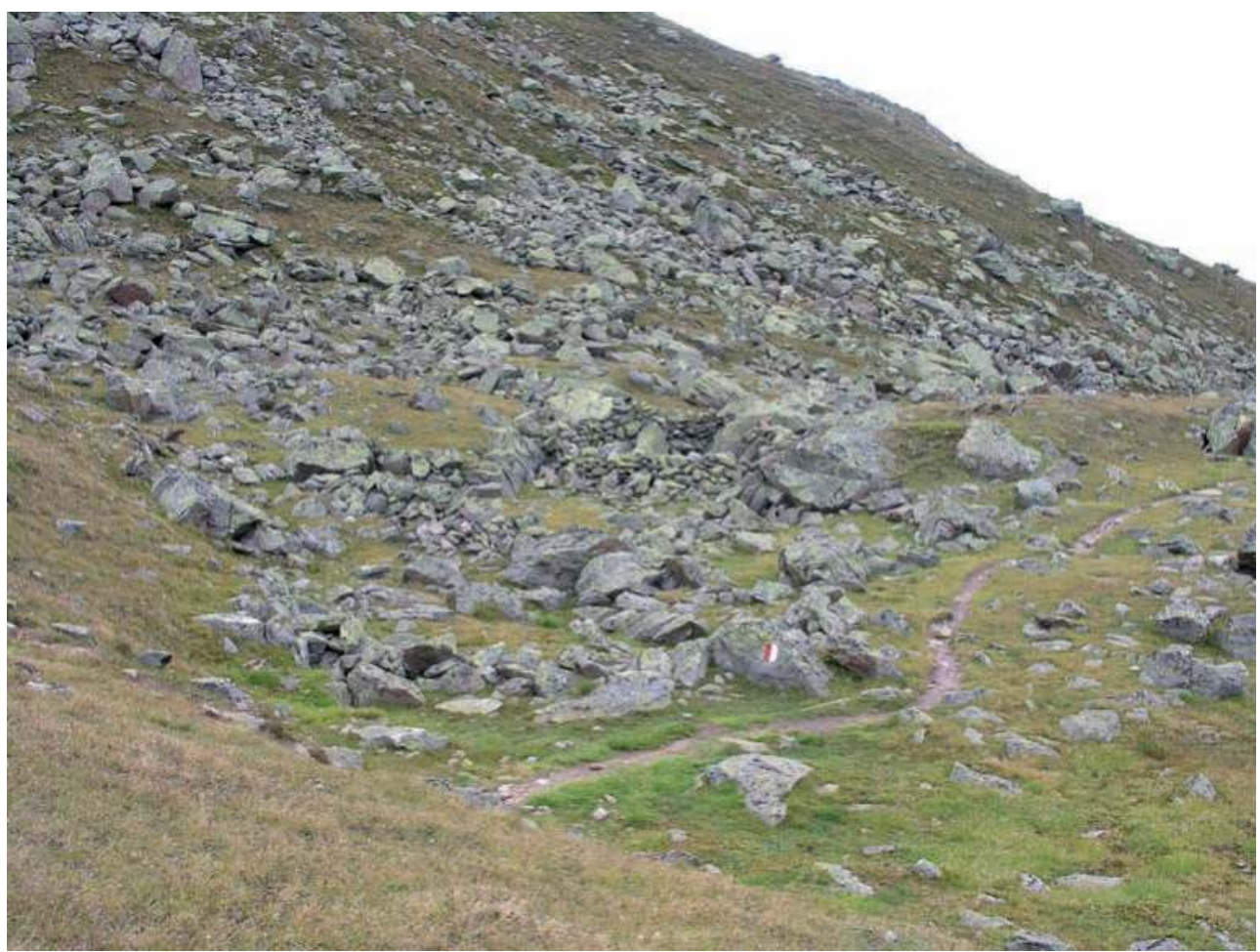

Abb. 6:

Dreifache Viehpferchanlage bei den „Haglen“ (Haage, Pferche) auf der Seenplatte, unterschiedliche Zerfallstadien (Foto: A. Zanesco)

reichen das Haupttal auf Höhe der alten Talschultern.

Der an sich bequemere Weg über das Timmelsjoch bedeutet aus dem Blickwinkel der Gebiete südlich und südöstlich (Passeiertal, Seewertal, Pfelderertal, Pfossental, Schnalstal, Vinschgau) einen weiten Umweg. Selbst aus dem Hinterpasseier und dem Pfelderertal ist das Gebiet bei geeigneten Verhältnissen rascher mittels der hoch gelegenen Übergänge erreichbar. Das zur Jagd, besonders aber wegen seiner Hochweiden günstige Obergurgl wird also seit dem Mesolithikum auch über diese Routen erschlossen worden sein. Welchen Stellenwert der Einzug von Norden (Inntal, äußeres Ötztal) her hatte, ist noch unklar. Auch wenn das Fundmaterial für eine Erschließung vorwiegend von Süden spricht, lässt die hohe Mobilität dieser Leute grundsätzlich alle Richtungen möglich erscheinen.

Andere von der Natur vorgegebene Wege entstehen aus in Talrichtung verlaufen- 


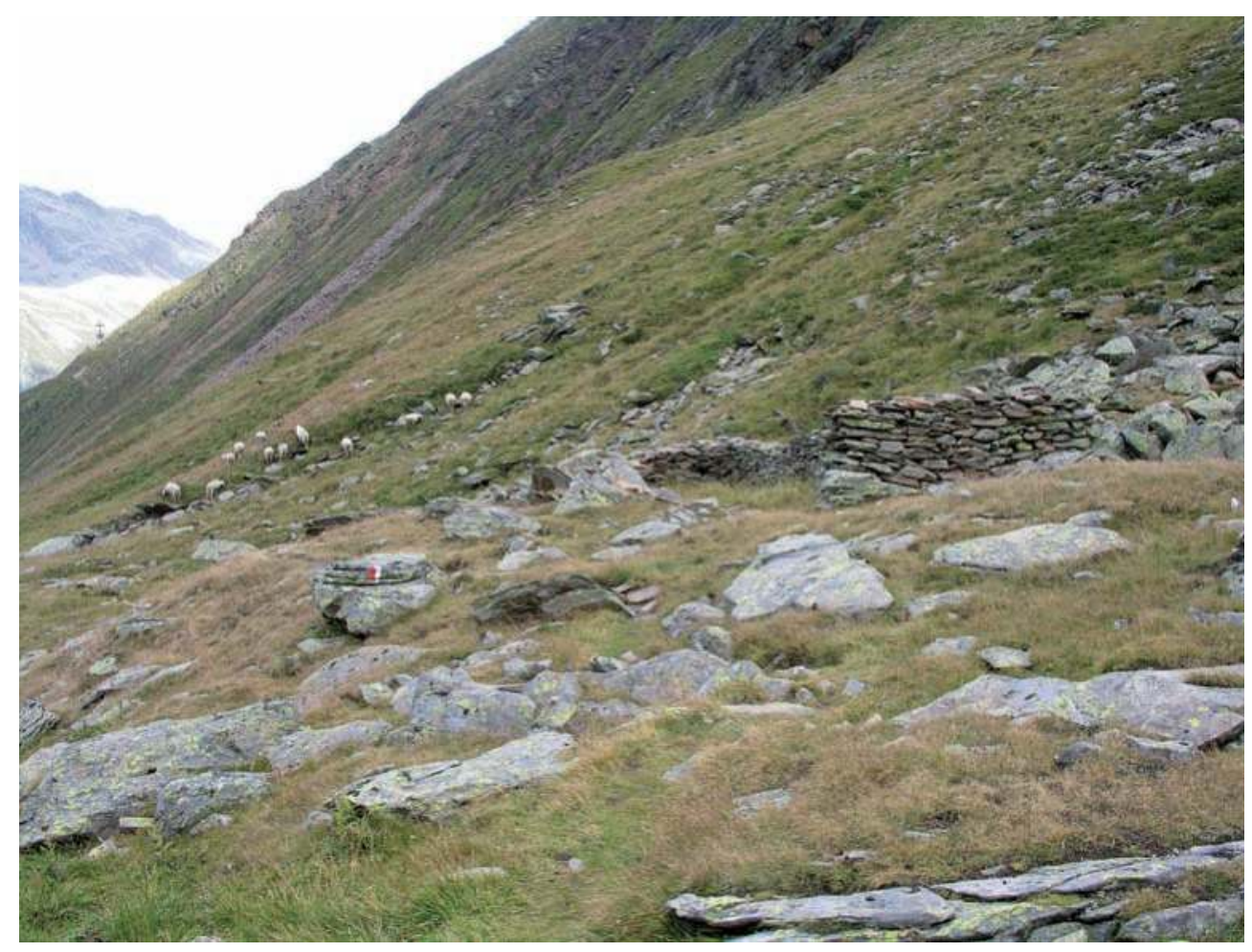

Abb. 7:

Verfallene Hütte (Stall?) im Königstal (Foto: A. Zanesco)

den Schultern, besonders jener über Am Beil-Küppele-Putzach, der im Talgrund auf den Gurgler Ferner trifft. Je nach Ankunftsrichtung und Ziel ist dieser Pfad oder jener an der gegenüberliegenden Talseite über die Gurgler Alm-Kleinalpl günstiger, um den Ferner zu erreichen. Hier konnte auf die jeweils andere Talseite übergewechselt werden. Dazu mochten auch den Gurgler Bach überspannende Lawinenkegel dienen, die den Sommer über liegen bleiben.

Auf die langgezogene „Seenplatte“, an der linken Talseite, etwa $2500 \mathrm{~m}$ hoch gele- gen, treffen keine Seitentäler. Der Pfad über die Gurgler Scharte ins Ventertal beginnt/endet im Bereich der Soomseen, etwa in der Mitte des Plateaus. Jener über das Ramoljoch trifft weit taleinwärts auf das Köpfle (3005 m). Funde fehlen hier bislang. Allerdings birgt die Seenplatte zahlreiche Relikte von Hirtenunterständen und Viehpferchen (Abb. 6), die teilweise in die mittlere bis späte Bronzezeit datieren (Gernot Patzelt, persönl. Mitteilung). Hier haben sich große Weideflächen entwickelt, die bereits für prähistorische Hirten von Bedeutung waren. 


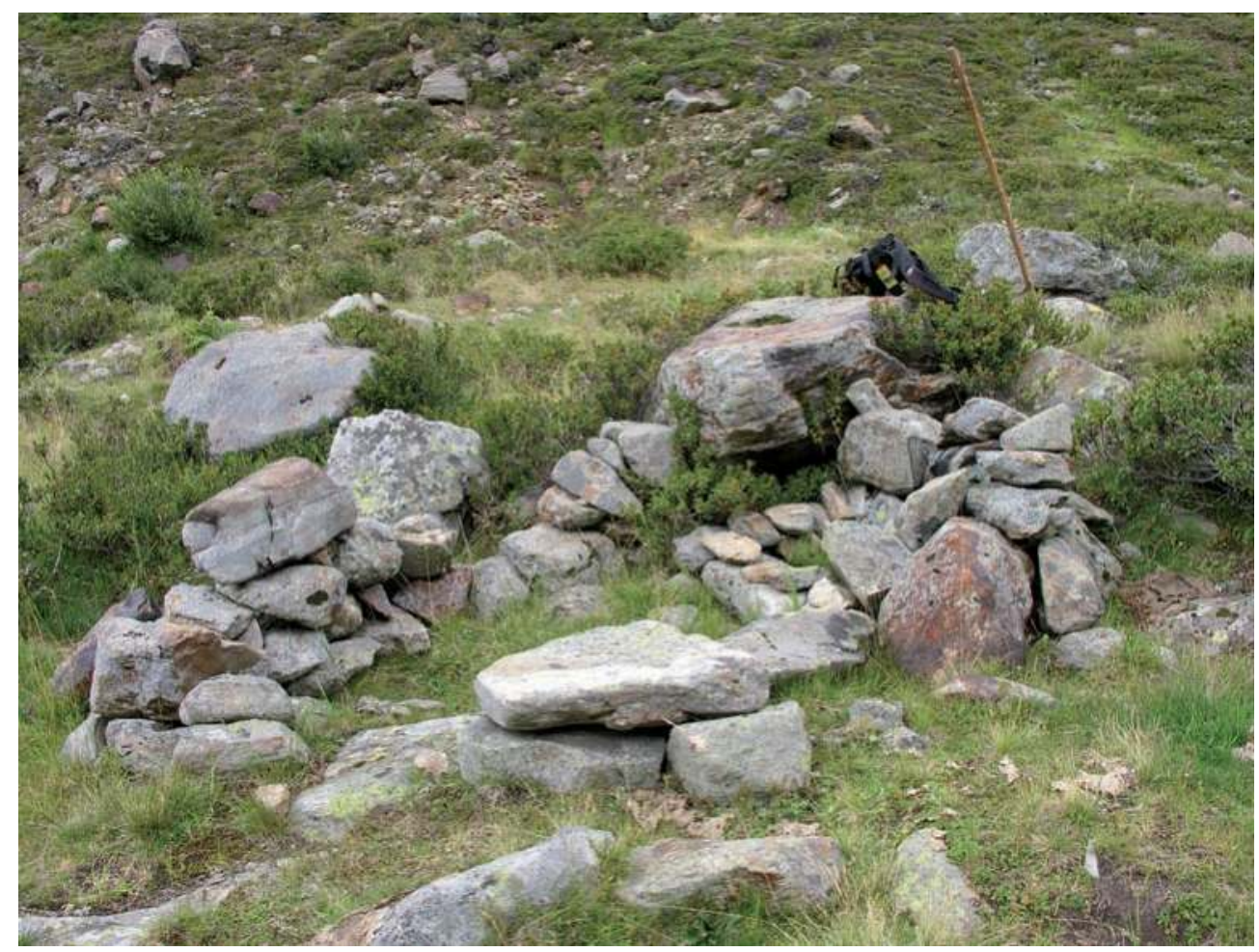

Abb. 8:

Verfallener Hirtenunterstand aus Trockenmauern im Timmelstal (Foto: A. Zanesco)

Fundstellen und Denkmäler

Potentielle Fundplätze sind nicht etwa die ausgesetzten Übergänge, sondern in der Nähe gelegene Kuppen, geschützte Senken und natürliche Unterstände, welche sich zur Rast eigneten. Darüber hinaus ist die Stufe oberhalb des Waldgrenzbereichs aufgrund der dort leichteren Fortbewegung für das Mesolithikum als primäre Funderwartungszone anzunehmen. Hier sind es v. a. die Talschultern säumende Kuppen und Blockhalden, welche La- gerplätze bieten. Über Silexfunde wird dementsprechend von Stellen berichtet, die am Ausgang der Seitentäler bzw. im Waldgrenzbereich liegen. $\mathrm{Zu}$ nennen sind Hochgurgl/Angern (Schäfer 2000, Schäfer 2001), Plattach/südliche Gurgler Heide (Schäfer 2000, Schäfer 2001) und Gurgler Alm (Rotmoostal).

Archäologische Sondagen durch D. Schäfer im Bereich Plattach (2260 m) am Ausgang des Gaisbergtales ergaben Gruben und Feuerstellen aus der älteren/mittleren Bronzezeit, der älteren-jüngeren Eisenzeit und der Römerzeit (1./2. Jh. n. Chr., 
Schäfer 2001). Dieser punktuelle Befund steht sicher stellvertretend für eine durchgehende und zunehmend intensivierte Nutzung dieser Gegend ab der Bronzezeit, wie sie auch in den Pollenprofilen zum Ausdruck kommt.

Die schon erwähnten Viehpferche und Schäferhütten finden sich abgesehen von der Seenplatte auch weiter taleinwärts (Putzach) bzw. auf den Böden zwischen Ferwall- und Königstal (Abb. 7) und im Timmelstal (Abb. 8). Sie werden teilweise bis heute genutzt (z. B. Salzstellen o. ä.) und der unterschiedliche Erhaltungsgrad sowie die bisherigen Radiokarbondatierungen lassen eine durchgehende Verwendung seit etwa 4000 Jahren möglich erscheinen. Als Denkmäler einer sehr lange währenden Landschaftsnutzung sollten sie genauer untersucht werden und vor allem erhalten bleiben.

Daneben finden sich zahlreiche bauliche Hinterlassenschaften unterschiedlicher Funktion. Meist handelt es sich um Almwüstungen (Abb. 2) und Infrastrukturbauten. In diesem Zusammenhang ist beispielsweise an Wegbefestigungen und Wasserhaltungsmaßnahmen zu denken. Zahlreiche Hüttenreste zeugen von der Alp- und Weidewirtschaft vergangener Jahrhunderte, von verfallenen Speicherbauten (Pillen) oder von der Kontrolle der seit 1918 nahe gelegenen Grenze (Zollhütten). Letztere Gebäude sind erst seit gut einem Jahrzehnt ihrer Funktion enthoben und vermutlich dem Verfall preisgegeben.

\section{Am Beilstein}

Am intensivsten wurde in Obergurgl bisher das Jägerlager am Beilstein ergraben, das sich in etwa 45 Minuten Fußmarsch vom Ort taleinwärts auf 2117 m Seehöhe befindet. Seine topographischen Bedingungen sind ungewöhnlich (Abb. 1-3). Auf der linken Talseite gelegen wird der Platz durch ein Knie des etwa 100 m tief einschneidenden Gurgler Baches nach Süden und Osten begrenzt. Westseitig schützt eine lange, etwa $3 \mathrm{~m}$ hohe Felswand. Nur nach Norden ist die Fläche offen, von wo man sie durch ein kleines Tal heraufkommend erreicht. Auf einer kleinen, erhöhten Fläche nahe der Schlucht bzw. an der Felswand finden sich die Spuren menschlicher Anwesenheit seit annähernd 10.000 Jahren.

Almgebäude des 12./13. und Mitte des 17. Jh. n. Chr. (Nicolussi et al. 2007) lehnen sich an den Fels und bedecken bzw. stören die prähistorischen Befunde. Doch hat sich an der Südseite ein starkes Paket mesolithischer/neolithischer Kulturschichten in einer ehemals natürlichen Senke erhalten (Abb. 4). Durch spätere Überdeckungen sind sie sehr gut geschützt. Sowohl die Kulturschichten als auch die Fundverteilung und eine Steinsetzung scheinen einen halbkreis- bis kreisförmigen Bereich zu begrenzen, der hauptsächlich benutzt wurde. Vorbehaltlich weiterer Auswertungen dürfte es sich dabei um eine Anlage aus Sitzsteinen und 


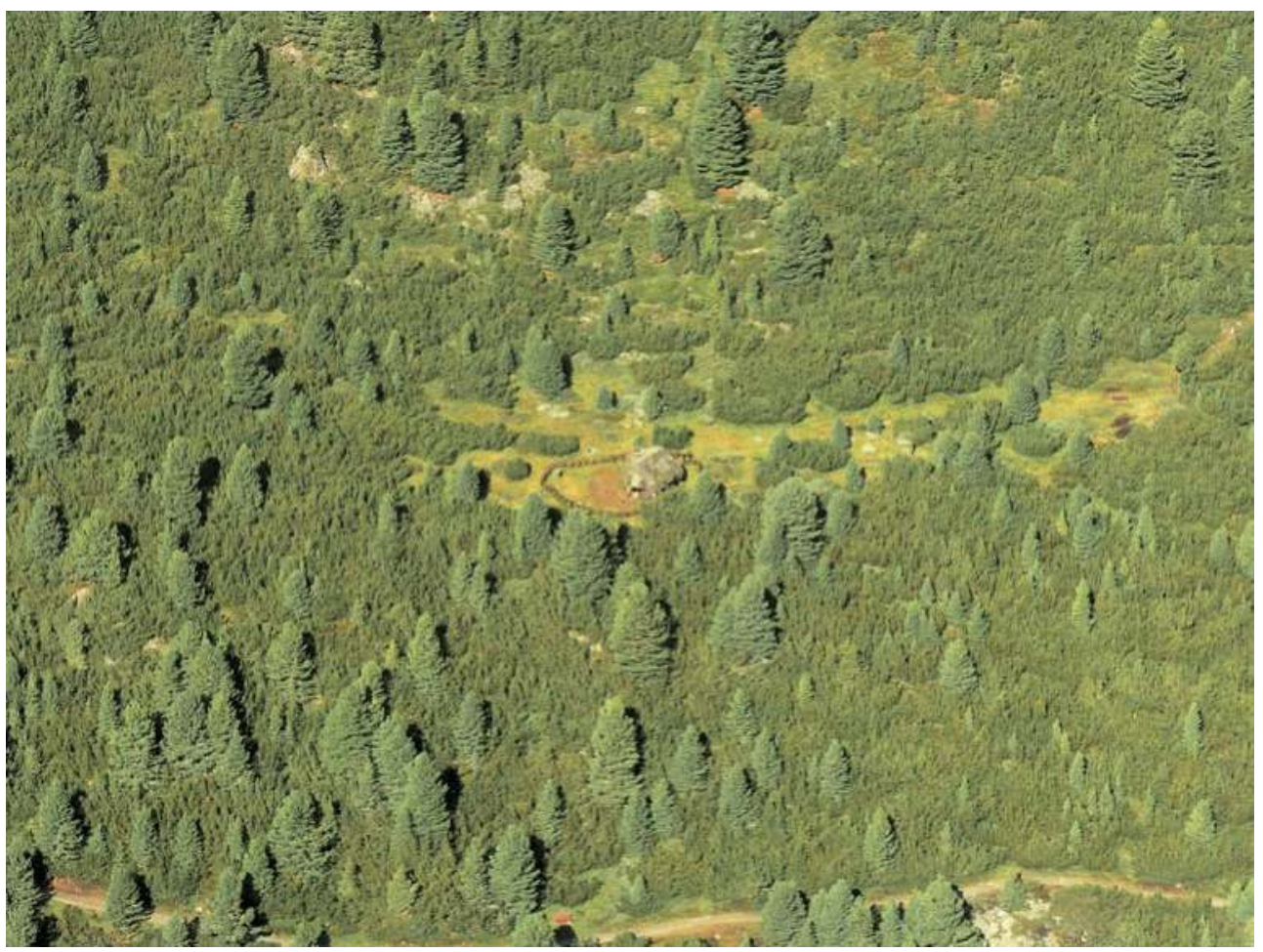

Abb. 9:

Blick auf den Hohlen Stein aus Richtung der Ramolalm in Vent (Foto: A. Zanesco)

anderen Bestandteilen (z. B. Pfostenloch) gehandelt haben, die nach Norden hin offen war.

Die Kulturschichten lieferten ${ }^{14} \mathrm{C}$-Daten ab der ersten Hälfte des 10. Jahrtausends BPcal. Weitere Feuerstellen ergaben Daten des 7. bis 3. Jahrtausends BPcal. Auch das Artefaktmaterial spricht für eine durchgehende Nutzung als Jagdplatz noch über diesen Zeitraum hinaus. Der Anteil der Geräte ist gering. Die große Masse der Funde besteht wie üblich aus Abschlägen und Absplissen. Nur sehr vereinzelt wurde auch Bergkristall verwendet.
Aufgrund der fortgesetzten Nutzung des Platzes und der relativ umfangreichen Strukturen sowie der Lage innerhalb des Waldbereiches kann man ihn am ehesten als Sekundärstation ansprechen, die als Ausgangslager für Jagdausflüge in die Gegend diente. Die nahebei gewonnenen Torfprofile lassen schließlich eine beständige Weidenutzung seit dem 4. Jahrtausend BPcal erkennen.

Ähnlich, wenn auch in einer anderen topographischen Situation dürfte das von W. Leitner ergrabene Jägerlager am Eingang des Rofentales bei Vent einzu- 
ordnen sein (Leitner 1999). Hier ist allerdings der Zusammenhang mit einer Wegkreuzung offensichtlich, der dem Platz einen entsprechend „kommunikativen" Charakter gegeben haben dürfte. Auch an dieser Stelle wurden Sitzsteine in Zusammenhang mit Feuerstellen freigelegt. Ein weiteres Beispiel ist schließlich der in der Nähe befindliche und ebenfalls von W. Leitner untersuchte Hohle Stein in Vent (Abb. 9). Besonders in der Trauflinie des auf einer kleinen Hangverebnung am Eingang ins Niedertal liegenden, stark überhängenden Felsblocks wurden Feuerstellen und Pfostenlöcher sowie zahlreiche mesolithische bis frühneolithische Artefakte festgestellt. Da dieser Platz am Weg durch das Niedertal liegt, der schließlich auch zum berühmten Fundort am Tisenjoch führt, dürfte es sich hier um einen häufig frequentierten Unterstand gehandelt haben. Eine kleine Fundkonzentration ist schließlich in der Flur Liechtl weiter taleinwärts zu vermelden (Blecha et al. 2008). Da hier schon öfter mesolithische Artefakte beobachtet wurden, ist von einem nahe gelegenen Lagerplatz auszugehen. Noch leer von prähistorischem Fundmaterial ist jedoch die sog. Kaser etwas weiter im Inneren des Tales, obwohl sie als größere offene Fläche sicher für gelegentliche Aufenthalte geeignet war (Zanesco 2007c).

Besonders prominent ist natürlich die Fundstelle beim Tisenjoch, wo 1991 die mumifizierte Leiche eines kupferzeitlichen Mannes mitsamt seiner Habe ge- borgen wurde. Der Übergang wurde aber auch Jahrtausende vorher und nachher benutzt, wie entsprechendes Fundmaterial aus den nachfolgenden Grabungen ergab (Bagolini et al. 1995).

Die vereinzelten prähistorischen Fundstellen im Raum Obergurgl deuten an, was bei umfangreicherer, gezielter Suche aufgedeckt werden könnte. Die Forschung konzentriert sich aber mit gutem Grund auf Beispiele, die stellvertretend für bestimmte Nutzungszeiträume und Nutzungsstrategien stehen. Eine sorgfältige Untersuchung solcher Plätze kann stellvertretend für andere, ähnlich gelagerte Situationen stehen. In diesem Sinn wird auch weiterhin in interdisziplinärer Weise geforscht, um den Zusammenhang von Landschaft, Klima und Kultur über die Jahrtausende besser zu verstehen.

\section{Literatur}

Adam A. M. (1996) Le fibule di tipo celtico nel Trentino. Patrimonio storico artistico del Trentino 19, Trento.

Bagolini B., Dal Ri L., Lippert A., Nothdurfter H. (1995) Der Mann im Eis: Die Fundbergung 1992 am Tisenjoch, Gem. Schnals, Südtirol (zweisprachig). In: Der Mann im Eis. Neue Funde und Ergebnisse, Spindler K., Rastbichler-Zissernig E., Wilfing H, Zur Nedden D., Nothdurfter H. (Hg.), Wien/New York: 3-52. 
Bazzanella M. (2001) The fauna of La Vela di Trento: preliminary analysis. Preistoria Alpina 34: 307-310.

Bazzanella M., Betti L., Wierer U. (2007) Mesolithic wetland exploitation at Galgenbühel/Dos de la Forca Italy, Eastern Alps. The fish fauna. In: The Role of Fish in Ancient Time, Plogmann H. H. (Hg.), Proceedings of the 13th Meeting of the ICAZ Fish Remains Working Group, Basel Rahden: 93-100.

Binsteiner A. (1994) Ausgewählte Silexlagerstätten und deren Abbau in den Provinzen Trient und Verona. Archäologisches Korrespondenzblatt 24: 255-263.

Blecha R., Cappelletto J. L. (2008) Fundberichte aus Österreich 46 (2007): $611 \mathrm{f}$.

Boschian G. (2005) Environment and Hunter-Gatherers Mobility in the Northern Adriatic Region. Preistoria Alpina 39: 91-102.

Bortenschlager S. (2000) The Iceman's environment. In: The Iceman and his $\mathrm{Na}$ tural Environment, Bortenschlager S., Oeggl K. (Hg.), Wien/New York: 11-24 Appendix 1-7.

Bortenschlager S. (2010) Vegetationsgeschichte im Bereich des Rotmoostales. In: Glaziale und periglaziale Lebensräume im Raum Obergurgl, Koch E.-M., Erschbamer B. (Hg.), Innsbruck: 77-91.

Crotti P. (2005) Mesolithic hunter-gatherers in western Switzerland: economy and mobility. Preistoria Alpina 39: 155-163.

Broglio A. (1994) Mountain sites in the context of the North-East Italian Upper
Palaeolithic and Mesolithic. Preistoria Alpina 28: 293-310.

Burga C. A. (2005) Die Schweizer Alpen als potenzieller Siedlungsraum im Spät- und Postglazial am Beispiel Graubündens. Preistoria Alpina 39: 31-37.

Dalmeri G., Lanzinger M. (1998) The evolution of the environment and human population of the Adige basin at the end of the late Ice Age and in the early Holocene. Preistoria Alpina 34 : 15-18.

Dalmeri G., Grimaldi S., Lanzinger M. (2000) Il paleolitico e il mesolitico. In: Storia del Trentino. T. 1. La preistoria e la protostoria, Il Mulino, Bologna: 11117, Fig. 20, 29, 30.

Dammers B. (2003) Das Mittelneolithikum in Rheinhessen. In: Leben und Sterben in der Steinzeit. Heide B. (Hg.), Zabern, Mainz: 101-130.

Geitner C., Schäfer D. (2010) Interdisziplinäre Zusammenarbeit an der Schnittstelle von Archäologie und Bodenkunde im Gebirge - Grundsätzliche Überlegungen und Beispiele des Mesolithfundplatzes Ullafelsen (Tirol). In: Archäologie in den Alpen. Alltag und Kult, Mandl, F., Stadler, H. (Hg.), ANISA, Gröbming, Haus im Ennstal, Nearchos 19: 25-40.

Gleirscher P. (2010). Hochweidenutzung oder Almwirtschaft? In: Archäologie in den Alpen. Alltag und Kult, Mandl, F., Stadler, H. (Hg.), Gröbming, Haus im Ennstal, Nearchos 19: 43-62.

Jochim M., Herhahn C., Starr H. (1999) The Magdalenian Colonization of Southern 
Germany. American Anthropologist, New Series 101: 129-142.

Joerin U. E., Nicolussi K., Fischer A., Stocker T. F., Schlüchter C. (2008) Holocene optimum events inferred from subglacial sediments at Tschierva Glacier, Eastern Swiss Alps. Quarternary Science Reviews 27: 337-350.

Kompatscher K., Hrozny-Kompatscher N. M. (2007) Dove piantare il campo: modelli insediativi e di mobilità nel Mesolitico in ambiente alpino. Preistoria Alpina 42: 137-161.

Madsen D. B., Scott T. R., Loosle B. (2000) Differential Transport Costs and HighAltitude Occupation Patterns in the Uinta Mountains, Northeastern Utah. In: Intermountain Archaeology, Madsen D. B., Metcalf M. D., University of Utah Anthropological Papers 122: 15-24.

Magny M., Leuzinger U., Bortenschlager S., Haas J. N. (2006) Tripartite climate reversal in Central Europe 5600-5300 years ago. Quaternery Research 65: 3-19.

Leitner W. (1999) Archäologische Forschungen im Ötztal. In: Die Gletschermumie aus der Kupferzeit. Schriften Südtiroler Archäologiemuseum, Bozen/Wien: 6979.

Lunz R. (1986) Vor- und Frühgeschichte Südtirols. Bd. 1, Steinzeit, Vallagarina, Bruneck.

Mandl F. (2003). Sölkpass. Ein 6000 Jahre alter Saumpfad über die Alpen. Anisa, Gröbming, Haus im Ennstal.
Mandl F., Stadler H. (2010). Archäologie in den Alpen. Alltag und Kult. Anisa, Gröbming, Haus im Ennstal, Nearchos 19.

Morris B. (2009) Insects as food among hunter-gatherers. Anthropology Today 24, Nr. 1: 6-8.

Moser H., Obex A. (1986) Mesolithische Silexgerätfunde im Texelgebiet. Der Schlern: 623-625.

Nicolussi K., Lumassegger G., Patzelt G. Thurner A. (2007) Heustadel im inneren Ötztal - dendrochronologische Ergebnisse zu einem vergehenden Element der alpinen Kulturlandschaft. In: Alpine Kulturlandschaft im Wandel. Hugo Penz zum 65. Geburtstag, Innsbrucker Geographische Gesellschaft (Hg.): 146-153.

Nicolussi K. (2009) Klimaentwicklung in den Alpen während der letzten 7000 Jahre. In: Prähistorische Besiedlung von zentralen Alpentälern in Bezug zur Klimaentwicklung, Oeggl et al. 2009a, alpine space - man \& environment, Vol. 6: Klimawandel in Österreich: 109-132.

Niederwanger G. (1984). Ur- und Frühgeschichte des Sarntales. Archäologische Forschungen in Tirol 8.

Niederwanger G. (2007) Steinzeitfunde am Timmelsjoch. Der Schlern 81, Heft 9: 32-37.

Oberrauch H., Niederwanger G. (2010) Archäologische Prospektionen im hinteren Passeiertal (Südtirol). In: Archäologie in den Alpen. Alltag und Kult, Mandl et al. (Hg.), Anisa, Gröbming, Haus im Ennstal, Nearchos 19: 167-198. 
Oeggl K. (2009) Prähistorische Landnutzung im mittleren Alpenraum. In: Die Geschichte des Bergbaus in Tirol und seinen angrenzenden Gebieten, Oeggl et al. (2009a), innsbruck university press, Innsbruck: 79-90.

Oeggl K., Prast M. (2009a) Die Geschichte des Bergbaus in Tirol und seinen angrenzenden Gebieten. Proceedings zum 3. Milestone-Meeting des SFB HiMAT vom 23.-26.10.2008 in Silbertal, Innsbruck.

Oeggl K., Nicolussi K. (2009b) Prähistorische Besiedlung von zentralen Alpentälern in Bezug zur Klimaentwicklung. Alpine Space - Man \& Environment 6. Klimawandel in Österreich, Innsbruck: 77-86.

Patzelt G., Kofler W., Wahlmüller B. (1997) Die Ötztalstudie - Entwicklung der Landnutzung. In: Alpine Vorzeit in Tirol. Begleitheft zur Ausstellung. Innsbruck: 46-62.

Patzelt G. (2000) Natürliche und anthropogene Umweltveränderungen im Holozän der Alpen. Rundgespräche der Kommission für Ökologie 18. Entwicklung der Umwelt seit der letzten Eiszeit: 119-125.

Patzelt G. (2008) Der Schwemmkegel von Hall. Forum Hall in Tirol 2: 12-21.

Pedrotti A. (2000) Il Neolitico. In: Storia del Trentino. T. 1. La preistoria e la protostoria, Bologna: 115-181.

Pedrotti A. (2001) The Neolithic Age in Trentino Alto Adige. Preistoria Alpina 34: 19-25.

Pedrotti A. (2009) Tradition and innovation between the Mesolithic and Early Neoli- thic in the Adige Valley (Northeast Italy. Documenta Praehistorica 36: 191-204.

Phoca-Cosmetaton N. (2006) Landscape use in Northeast Italy during the Upper Palaeolithic. Preistoria Alpina 41 23-49.

Rachoud-Schneider A.-M., Praz J.-C. (2002) Klima und Vegetation. Das Rhonetal als Beispiel. In: Die ersten Menschen im Alpenraum. Ausstellungskatalog Sitten 2002, Sitten: 19-37.

Reitmaier T. (2010) Letzte Jäger, erste Hirten. Hochalpine Archäologie in der Silvretta. Begleitheft zur Ausstellung 2010. Zürich.

Shackleton J. C., Van Adel T. H., Runnels C. N. (1984) Coastal Paleogeography of the Central and Western Mediterranean during the Last 125,000 Years and Its Archaeological Implications. Journal of Field Archaeology 11: 307-314.

Schäfer D. (1999) Jahresbericht 1999, Archäologische Hochgebirgsforschung, Universität Innsbruck: http://www. hochgebirgsarchaeologie.at.

Schäfer D. (2000) Jahresbericht 2000, Archäologische Hochgebirgsforschung, Universität Innsbruck: http://www. hochgebirgsarchaeologie.at.

Schäfer D. (2001) Jahresbericht 2001, Archäologische Hochgebirgsforschung, Universität Innsbruck: http://www. hochgebirgsarchaeologie.at.

Schäfer D. (2011) Archäologische Hochgebirgsforschung, Universität Innsbruck http://www.hochgebirgsarchaeologie.at.

Schweizer M. (2002) Die urgeschichtliche Fauna. In: Die ersten Menschen im Al- 
penraum. Ausstellungskatalog Sitten 2002, Sitten: 39-53.

Spindler K. (2005) Transhumanz. Preistoria Alpina 39: 219-225.

Testart A. (1982) The Significance of Food Storage among Hunter-Gatherers: Residence Patterns, Population Densities, and Social Inequalities. Current Anthropology 23: 523-537.

Töchterle U. (2009) Kontinuität und Wandel: Funde aus prähistorischer Zeit. In: Archäologische Topographie der Siedlungskammer Ampass. Grabherr G., Kainrath B. (Hg.), IKARUS 4: 29-244.

Vorren K.-D., Mørkved B., Bortenschlager S. (1993) Human impact on the Holocene forest line in the Central Alps. Vegetation History and Archaeobotany 2: 145 156.

Wahlmüller N. (1985) Der vorgeschichtliche Mensch in Tirol. Neue Aspekte aufgrund der Pollenanalyse. Veröffentlichungen des Ferdinandeums. 65: 105-120.

Watanabe H. (1973). The Ainu Ecosystem. Environment and Group Structure.
American Ethnological Society. Monograph 54. 2. A., Washington.

Zanesco A. (2007a) Fundberichte aus Österreich 45 (2006): 627-629.

Zanesco A. (2007b) Fundberichte aus Österreich 45 (2006): 673f.

Zanesco A. (2007c) Fundberichte aus Österreich 45 (2006): 770-772.

Zanesco A. (2008) Mensch und Landschaft bis zur Ankunft der Römer. In: Absam, Laimgruber M., Moser H., Steindl P. (Hg.), Gemeinde Absam, Absam: 59-82.

\section{Verzeichnis des Autors}

Alexander Zanesco

Institut für Archäologien

Universität Innsbruck

Langer Weg 11, 6020 Innsbruck

Alexander.Zanesco@uibk.ac.at 


\title{
Kapitel 4 I Lärchen-Zirbenwälder und Zwergstrauch- heiden
}

\author{
Roland Mayer, Brigitta Erschbamer
}

\section{Zusammenfassung}

Lärchen-Zirbenwälder und Zwergstrauchheiden sind landschaftsprägende Vegetationstypen der subalpinen Stufe im inneren Ötztal. Der Obergurgler Zirbenwald - ein alter, einheitlich aufgebauter Waldbestand in der Terminalphase - kann dem VaccinioPinetum cembrae nardetosum zugeordnet werden. Je nach Kronenschluss und Meereshöhe lassen sich eine Ausbildung mit Oxalis acetosella (dichterer Kronenschluss, $\leq 2060 \mathrm{~m}$ Meereshöhe) und eine Ausbildung mit Loiseleuria procumbens (lockerer Kronenschluss, $\geq 2100 \mathrm{~m}$ Meereshöhe) differenzieren. Die Zwergstrauchbestände im Bereich der Gurgler Heide unterscheiden sich hinsichtlich Wuchshöhe und Mikrorelief deutlich voneinander: in Muldenlagen dominiert das hochwüchsige Rhododendretum ferruginei und an Windkanten das Loiseleurio-Cetrarietum. Die Veränderungen der Vegetation und der Artenzahl wurden in $1 \mathrm{~m}^{2}$-Dauerflächen in drei Typen von Zwergstrauchheiden von 2000-2008 untersucht. Mit Ausnahme des von Flechten dominierten LoiseleurioCetrarietums wurden in diesem Zeitraum jeweils sehr deutliche Veränderungen fest- gestellt. Mögliche Ursachen dafür sind Biomassenzuwachs infolge von Klimaerwärmung oder Stickstoffeintrag direkt aus der Atmosphäre. Das Befahren mit Skiern wirkte sich ebenfalls auf die Artenzusammensetzung aus.

\section{Abstract}

Cembran pine forests and dwarf shrub heaths are characteristic plant communities in the subalpine zone of the inner Oetz valley. In Obergurgl, a homogenously structured pine forest consists of old pines (Pinus cembra) in the terminal stage. The community was defined as Vaccinio-Pinetum cembrae nardetosum. According to tree crown cover and altitude, two variants were distinguished: a variant with Oxalis acetosella (higher tree crown cover, $\leq 2060 \mathrm{~m}$ a.s.l.) and a variant with Loiseleuria procumbens (lower tree crown cover, $\geq 2100 \mathrm{~m}$ a.s.l). The dwarf shrub heaths can be differentiated by canopy height and microrelief. They were attributed to the Rhododendretum ferruginei in troughs and the Loiseleurio-Cetrarietum on wind-exposed edges. Additionally, species composition and 
number of three dwarf shrub communities were studied for nine years (2000-2008) in $1-\mathrm{m}^{2}$ permanent plots. With the exception of the wind-exposed Loiseleurio-Cetrarietum, significant changes were detected in the dwarf shrub communities, probably caused by global climate warming, atmospheric nitrogen deposition and skiing.

\section{Einleitung}

Lärchen-Zirbenwälder (= Lärchen-Arvenwälder) sind typisch für die hochsubalpine Stufe und den Waldgrenzbereich der Innen- und zum Teil auch der Zwischenalpen. Die hochsubalpine Stufe erstreckt sich in den Innenalpen (kontinentale Kernzone) von 2000-2300 m Meereshöhe (Kilian et al. 1994), wobei die potenzielle Waldgrenze bei 2200-2350 m anzusetzen ist (Schiechtl \& Stern 1975, Bortenschlager 1993). Sie wurde jedoch durch massive menschliche Eingriffe um ca. 200-300 m erniedrigt. Im inneren Ötztal liegt die aktuelle Waldgrenze heute daher bei ca. 2100-2200 m Meereshöhe (Rybníček \& Rybníčková 1977). Die höchstgelegenen Zirben stehen am Schönwieskopf bei knapp 2300 m Meereshöhe.

Lärche (Larix decidua) und Zirbe (Pinus cembra) sind weit besser an das extreme Klima im Waldgrenz-Ökoton angepasst als etwa die Fichte (Picea abies), welche in der tiefsubalpinen Stufe der Innen- alpen (1700-2000 m) Wälder mit höherem Anteil an Lärche und Zirbe ausbildet (Kilian 1994). Die Frostresistenz (nach natürlicher Frostabhärtung) liegt für die Zirbe bei $-42^{\circ} \mathrm{C}$ bis $-54^{\circ} \mathrm{C}$, während für die Fichte $-38{ }^{\circ} \mathrm{C}$ bis maximal $-45^{\circ} \mathrm{C}$ angegeben sind (Zitate in Neuner 2007). Hinsichtlich des Ausgangsgesteins ist die Zirbe ziemlich bodenvag (Ellenberg \& Leuschner 2010). Aufgrund ihres Schwerpunktes in den Innenalpen sind es hauptsächlich silikatische Ausgangsgesteine, auf denen sie stockt. Sie gedeiht aber auch auf Karbonatgestein, so z.B. auf Hartkalken und Dolomit. Diese Karbonat-LärchenZirbenwälder (Rhododendro hirsutiPinetum cembrae; Bojko 1931, Karner \& Willner nom. nov.) kommen vor allem in den Rand- und Zwischenalpen von 1700 $\mathrm{m}$ bis maximal $2100 \mathrm{~m}$ Meereshöhe vor (Wallnöfer 1993).

Lärchen-Zirbenwälder wurden vom Menschen durch umfangreiche Rodungen und Almwirtschaft im Lauf von Jahrhunderten drastisch verändert. Im Obergurgler Zirbenwald wurden die ersten Brandhorizonte ins Neolithikum (4000-4230 v. Chr.) datiert (Patzelt 1997). Wiederholte Brandrodung scheint es in Obergurgl seit der Bronzezeit gegeben zu haben (Aulitzky et al. 1963). Generell erfolgten großflächige Eingriffe in Lärchen-Zirbenwälder vor allem im Mittelalter, als diese zum Eigentum der bajuwarischen Siedler gehörten. Zu den am stärksten nachteiligen Maßnahmen im Zuge der Almwirtschaft gehörten Brandrodung für die Weidege- 
winnung, Abholzung der Samenbäume sowie Waldweide und Streugewinnung, wodurch dem Bergwald Nährstoffe entzogen wurden (Reisigl \& Keller 1999). Nach einer Rodung oder einer natürlichen Katastrophe, wie etwa einem Waldbrand, benötigt eine Wiederbewaldung in der subalpinen Stufe viele Jahrzehnte, wobei die Entwicklung ausgehend von einer lärchenreichen Initialphase über eine Phase mit Lärche und Zirbe hin zu einer zirbenreichen Terminalphase verläuft (Mayer 1986). Das Erreichen der Terminalphase ist erst nach 300-400 Jahren zu erwarten (Mayer 1974).

An die Stelle der Lärchen-Zirbenwälder traten großflächig Ersatzgesellschaften, wie etwa Zwergstrauchheiden, Almwiesen und -weiden. Die Beweidung seit dem Neolithikum veränderte wohl auch die Zusammensetzung der von der Brandrodung verschonten Lärchen-ZirbenwaldBestände und trug zu ihrer Dezimierung bzw. Degradierung wesentlich bei. In den Alpentälern sind daher heute nur mehr kleine Reste von einigermaßen intakten Lärchen-Zirbenwäldern vorhanden. In Tirol nahm im 20. Jahrhundert die Fläche, die von Lärchen-Zirbenwäldern bestockt war, von mehr als 100.000 ha auf nur noch 25.000 ha ab. Im Wuchsgebiet Innenalpen reduzierte sich diese Fläche sogar von 25.000 ha auf nur noch 5.000 ha. Das entspricht einem enormen Flächenverlust von $75-80 \%$ gegenüber der ursprünglichen Bewaldung (Klosterhuber $\&$ Hotter 2001). Davon weitgehend ver- schont blieb der Obergurgler Zirbenwald, der insgesamt etwa 20 ha umfasst und auf Grund seiner Geschlossenheit und seines Alters im Jahr 1963 als Naturdenkmal unter Schutz gestellt wurde (Moser 1973). Wesentlich weiter verbreitet als LärchenZirbenwälder sind heute Zwergstrauchheiden, die gelegentlich noch sehr locker mit Zirben bestockt sind. Die ausgedehnten Bestände in Obergurgl sind zum Großteil durch die menschliche Nutzung der Waldgrenzlagen bedingt. Seit Mitte des 19. Jahrhunderts, aber im Besonderen seit 1950, veränderte sich die Landnutzung in der subalpinen Stufe gravierend. Die Almwirtschaft wurde immer weniger rentabel, sodass viele Almwiesen- und weiden im gesamten Alpenraum aufgelassen wurden. Dadurch dehnte sich das Areal der Zwergstrauchheiden aus. Heute scheinen sich auch die Bestände der Zirbe wieder zu regenerieren (Didier 2001, Motta et al. 2006, Casalegno et al. 2010, Ellenberg \& Leuschner 2010).

Der Zirbenwald und die Zwergstrauchheiden im Raum Obergurgl wurden von Pitschmann et al. (1980) im Maßstab 1 : 37.500 sowie im Zuge der Vegetationskartierung des Ruhe- und Natura 2000-Gebietes Ötztal kartiert (Lumasegger et al. 2009). Eine detaillierte und umfassende pflanzensoziologische Bearbeitung fehlte jedoch bisher. Die Veränderungen der Artenzusammensetzung und der -vielfalt von Zwergstrauchheiden in Obergurgl werden seit dem Jahr 2000 im Rahmen eines Monitoring-Programmes beobach- 
tet (Kaufmann 2005). Ihre Dynamik im Lauf der Zeit und vor allem der Einfluss des Klimawandels sollen langfristig erfasst werden. Ziel der vorliegenden Arbeit ist es, den Obergurgler Zirbenwald und die Zwergstrauchbestände auf der Gurgler Heide pflanzensoziologisch zu beschreiben und zu klassifizieren. Zudem soll analysiert werden, wie sich Artenzusammensetzung und -vielfalt von verschiedenen Zwergstrauchbeständen im Vergleich zwischen den Jahren 2000 und 2008 in $1 \mathrm{~m}^{2}$ großen Dauerflächen verändert haben.

\section{Untersuchungsgebiet}

Das Untersuchungsgebiet für die vorliegende Arbeit liegt im innersten Ötztal auf der orographisch rechten Talseite des Gurglertales oberhalb von Obergurgl. Geologisch betrachtet gehört es zum südlichen Ötztal-Stubai-Komplex, in welchem altkristalline Silikatgesteine vorherrschen (Purtscheller 1978). Hauptgesteine sind Biotit-Plagioklasgneis ( $\mathrm{Pa}$ ragneis) und mineralische Glimmerschiefer. Vereinzelt kommen auch Quarzite mit geringer Mächtigkeit vor (Purtscheller 1978). Einen detaillierten Überblick über die Geologie und Geomorphologie im Raum Obergurgl gibt Krainer (2010). Das Untersuchungsgebiet stellt durchwegs potentielles Waldgebiet dar. Ausgenommen davon sind lediglich die stark von Winden beeinflussten, exponierten felsigen Hangbereiche. Der Schwerpunkt des Lärchen-Zirbenwaldes liegt zwischen 1900 und 2200 m Meereshöhe (Pitschmann et al. 1980). Unterhalb von $1700 \mathrm{~m}$ ist auch die Fichte beigemischt. Lumasegger et al. (2009) wiesen den hochsubalpinen Lärchen-Zirbenwald gemeinsam mit dem Lärchenwald als den flächengrößten Waldtyp im Ruhe- und Natura 2000-Gebiet Ötztaler Alpen aus (1,6 \% Flächenanteil aller Lebensraumtypen; s. Abb. 1 in Kapitel 1).

Auch die Zwergstrauchheiden wurden im inneren Ötztal großflächig kartiert. Für das Gurglertal typisch sind das Rhododentretum ferruginei, das Empetro-Vaccinietum gaultherioides, das JuniperoArctostaphyletum und das LoiseleurioCetrarietum. Diese Gesellschaften bevorzugen die Hangbereiche zwischen 2000 und $2400 \mathrm{~m}$ Meereshöhe und sind im gesamten Ruhe- und Natura 2000-Gebiet Ötztaler Alpen sehr weit verbreitet. Sie nehmen hier rund $7 \%$ der kartierten Lebensräume ein (Lumasegger et al. 2009).

Das Untersuchungsgebiet der vorliegenden Studie lässt sich in zwei Teilgebiete untergliedern. Das erste Teilgebiet umfasst die zusammenhängende Waldfläche des Obergurgler Zirbenwaldes (19302060 m Meereshöhe) sowie die stark aufgelockerten Zirben-Bestände und Zwergstrauchheiden oberhalb von 2100 $\mathrm{m}$ Meereshöhe (Abb. 1). Es befindet sich ca. $1,5 \mathrm{~km}$ in südwestlicher Richtung vom Ortszentrum Obergurgls entfernt. 


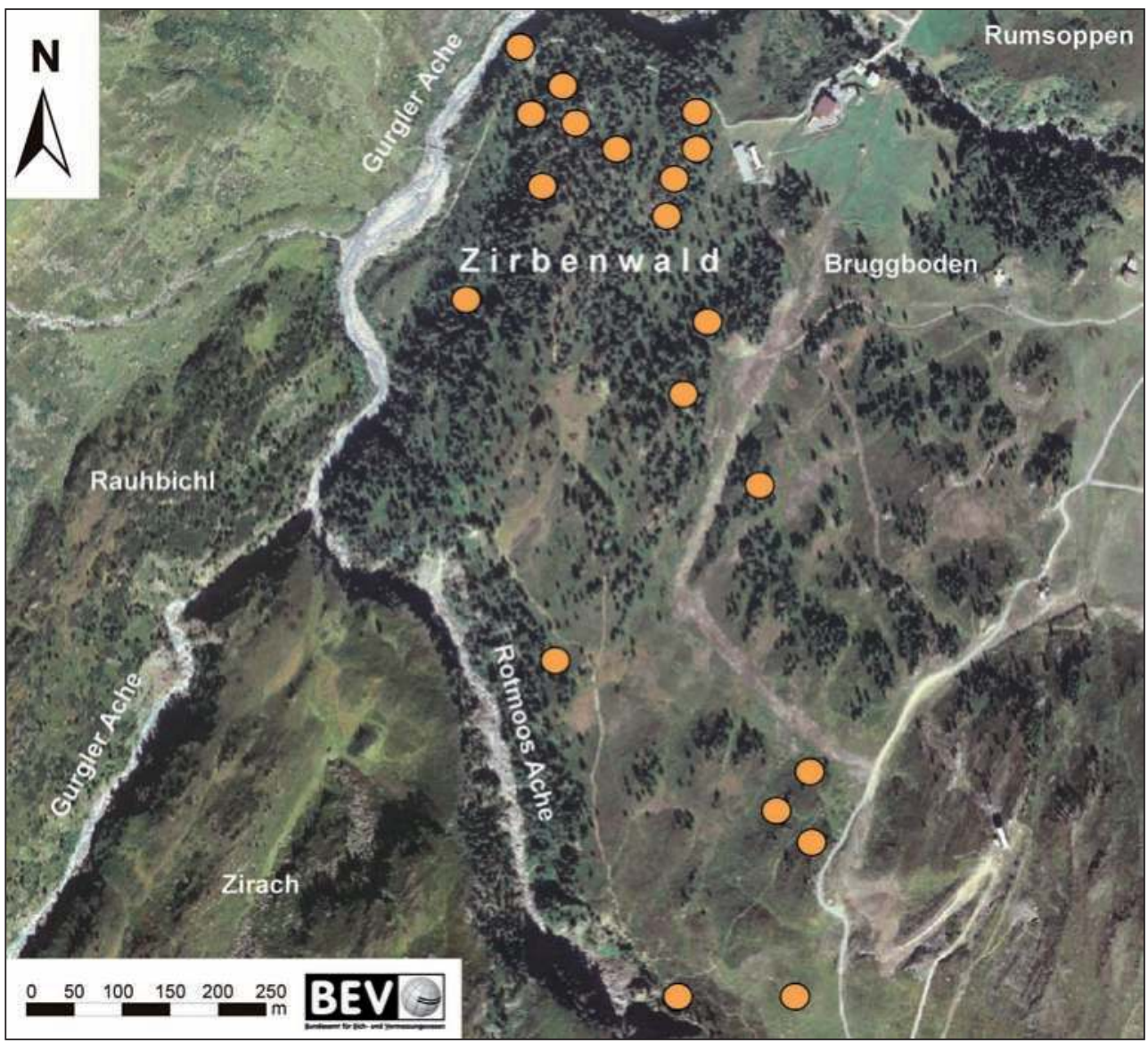

Abb. 1:

Die Position der 20 Aufnahmeflächen (Kreise) im Obergurgler Zirbenwald. (Karte: Tiroler Landesregierung, (C BEV 2011, Vervielfältigt mit Genehmigung des BEV - Bundesamtes für Eich- und Vermessungswesen in Wien, T2011/80692)

Die Hänge sind meist zwischen $18^{\circ}$ und $43^{\circ}$ geneigt und in WNW- bis seltener in NW-Richtung exponiert. Das zweite Teilgebiet befindet sich auf der Gurgler Heide, wo niedrigwüchsige, spalierartige Zwergstrauchheiden an Windkanten (um ca. $10 \mathrm{~cm}$ Wuchshöhe) gedeihen. Es handelt sich um ein Loiseleurio-Cetrarietum, bei welchem die Flechten eine sehr große Rolle spielen. Zudem kommen auch hochwüchsige Zwergstrauchbestände (ca. $50 \mathrm{~cm}$ Wuchshöhe) in Hangmulden oder auf Blockschutt vor (Abb. 2). Diese werden von Rhododendron ferrugineum (Rostrote Alpenrose) beherrscht. Am Rumsoppen dominiert Empetrum hermaphrodi- 


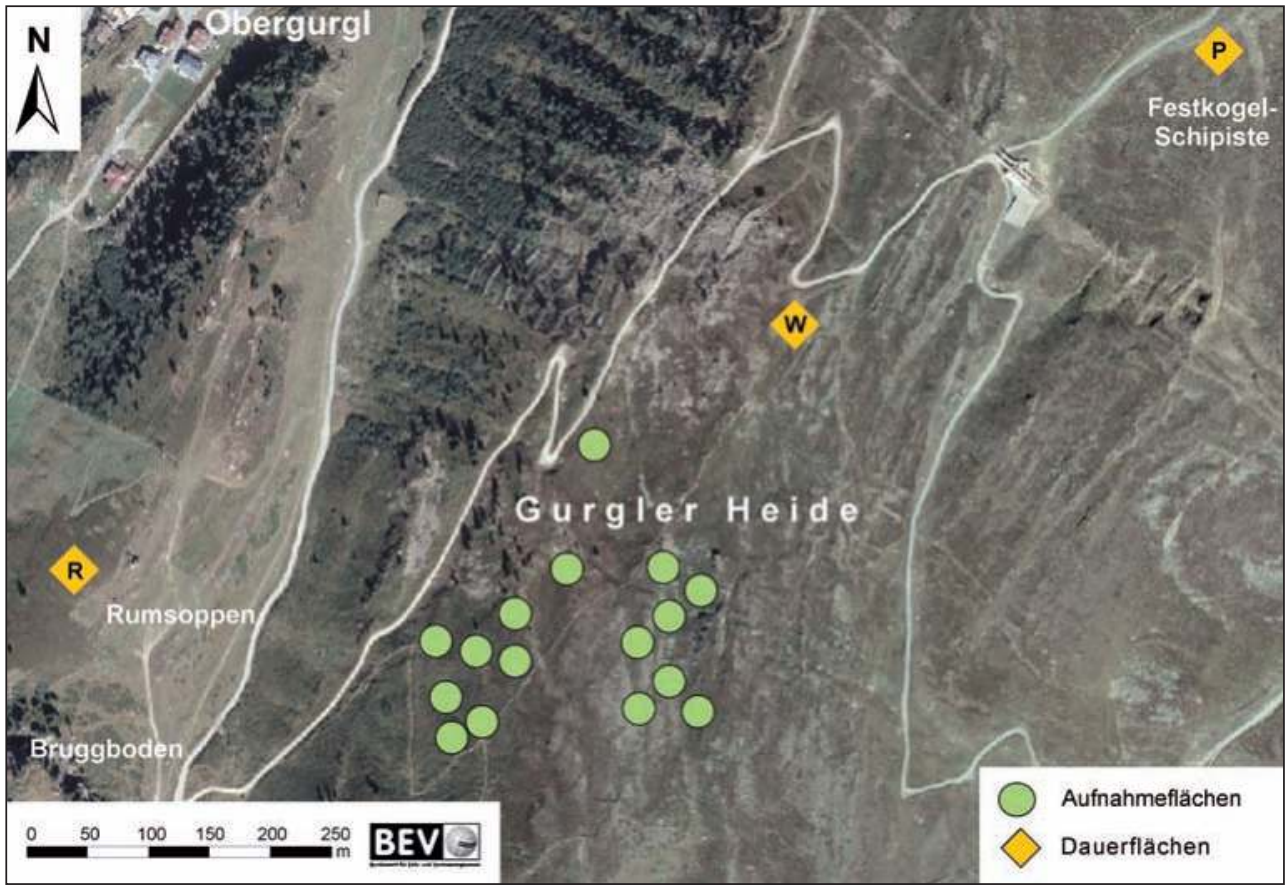

Abb. 2:

Die Position der 16 Aufnahmeflächen (Kreise) und die Lage der drei Standorte mit $1 \mathrm{~m}^{2}$-Dauerflächen (Rauten) im Bereich der Gurgler Heide; R: Rumsoppen, W: Windheide, P: Pistenheide (Karte: Tiroler Landesregierung, (C) BEV 2011, Vervielfältigt mit Genehmigung des BEV - Bundesamtes für Eich- und Vermessungswesen in Wien, T2011/80692)

tum (Zwitter-Krähenbeere) zusammen mit Vaccinium gaultherioides (Rauschbeere), $V$. vitits-idaea (Preiselbeere) und $V$. myrtillus (Heidelbeere). Es gedeihen jedoch auch dort immer wieder Büsche von Rhododendron ferrugineum und prägen so das Erscheinungsbild von weitem. Im Bereich der Festkogel-Schipiste kommen Zwergstrauchheiden am Pistenrand auf 2350 m Meereshöhe vor. Diese Pistenheide wird von Schifahrern genutzt aber nicht mit Pistenfahrzeugen präpariert. Es dominieren Vaccinium vitis-idaea und
Loiseleuria procumbens (Gamsheide), Empetrum hermaphroditum ist beigemischt. Auch Carex curvula (Krumm-Segge) und die Flechte Cetraria islandica (Isländisches Moos) sind auffällig. Dieses zweite Teilgebiet ist ca. $1 \mathrm{~km}$ in südöstlicher Richtung vom Ortszentrum Obergurgls entfernt. Die Hänge sind überwiegend zwischen $16^{\circ}$ und $47^{\circ}$ geneigt und in WNW- bis vereinzelt in NW-Richtung exponiert.

In drei weiteren Zwergstrauchheiden im Untersuchungsgebiet wurden im Jahr 2000 im Rahmen des Langzeit-Monito- 
ring-Programmes (Kaufmann 2005) 1 $\mathrm{m}^{2}$ große Dauerflächen angelegt und in den Jahren 2000, 2003, 2004 und 2008 analysiert (Kaufmann 2005, R. Mayer unpubl. Daten). Diese drei Standorte befinden sich auf dem Rumsoppen (2040 m Meereshöhe, n=6), auf der Gurgler Heide (Windheide, $2300 \mathrm{~m}$ Meereshöhe, $\mathrm{n}=4$ ) und unmittelbar außerhalb der FestkogelSchipiste (Pistenheide, $2350 \mathrm{~m}$ Meereshöhe, $\mathrm{n}=3$; Abb. 2). Dort wurden jeweils auch Mikroklimamessungen durchgeführt. Dazu wurde je ein Datenlogger (Onset, Pocasset MA) verwendet, um die Luft- (200 cm über dem Boden) und die Bodentemperatur (10 cm Bodentiefe) zu messen. Die mittlere Jahres-Lufttemperatur war am Rumsoppen am höchsten (Abb. 3a). In der Windheide und beson- ders außerhalb der Festkogel Schipiste (Pistenheide) war sie deutlich niedriger (Abb. 3a). Die mittlere Jahres-Lufttemperatur war im Jahr 2005 deutlich am niedrigsten, die höchsten Werte wurden 2002 und 2006 erreicht. Die mittlere maximale Lufttemperatur wies im Jahr 2003 an allen Standorten einen außergewöhnlich hohen Wert auf (Abb. 3b). Die mittlere minimale Bodentemperatur im Winter war am Rumsoppen am höchsten und wies die geringsten Schwankungen von Jahr zu Jahr auf (Abb. 3c). Im Bereich der Windheide hingegen wurden die geringsten mittleren Minima der Bodentemperaturen im Winter gemessen und diese zeigten auch die deutlich größten jährlichen Schwankungen (Abb. 3c).

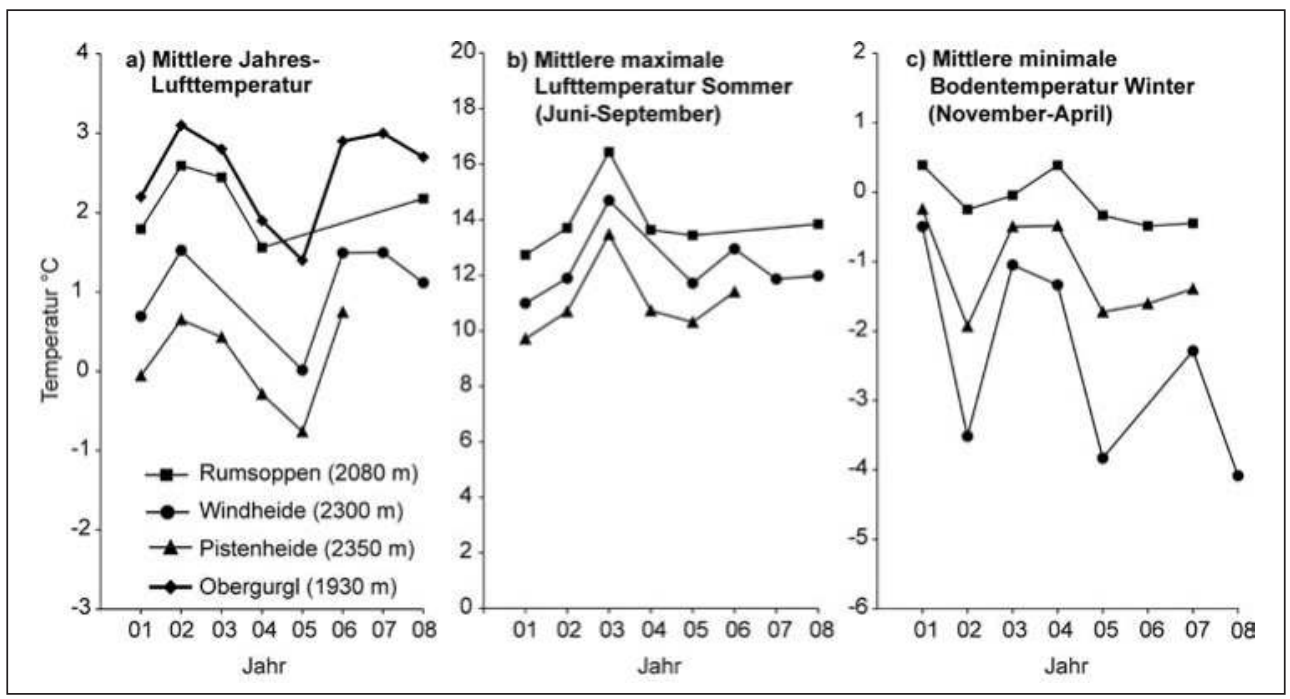

Abb. 3:

Das Mikroklima (Luft- und Bodentemperatur) an den drei Standorten mit Dauerflächenbeobachtungen. Als Referenz ist die mittlere Jahres-Lufttemperatur der Wetterstation in Obergurgl $(1930 \mathrm{~m})$ dargestellt. 


\section{Methoden}

\section{Vegetationsaufnahmen}

Um den Obergurgler Zirbenwald (Abb. 4, 5) und die Zwergstrauchheiden im Bereich der Gurgler Heide pflanzensoziologisch zu untersuchen, wurden im August 2010 insgesamt 36 Aufnahmen gemacht. In den Aufnahmeflächen wurden alle Gefäßpflanzen sowie die auffälligsten Moose und Flechten erfasst und ihre Artmächtigkeit mit Hilfe der kombinierten Abundanz-Dominanz-Skala von BraunBlanquet (1964), erweitert nach Reichelt \& Wilmanns (1973), geschätzt. Die Rindenmoose und die Krustenflechten auf den Felsen wurden nicht berücksichtigt. Im Obergurgler Zirbenwald wurden 20 Aufnahmen entlang eines Höhengradienten von 1950 bis 2160 m Meereshöhe

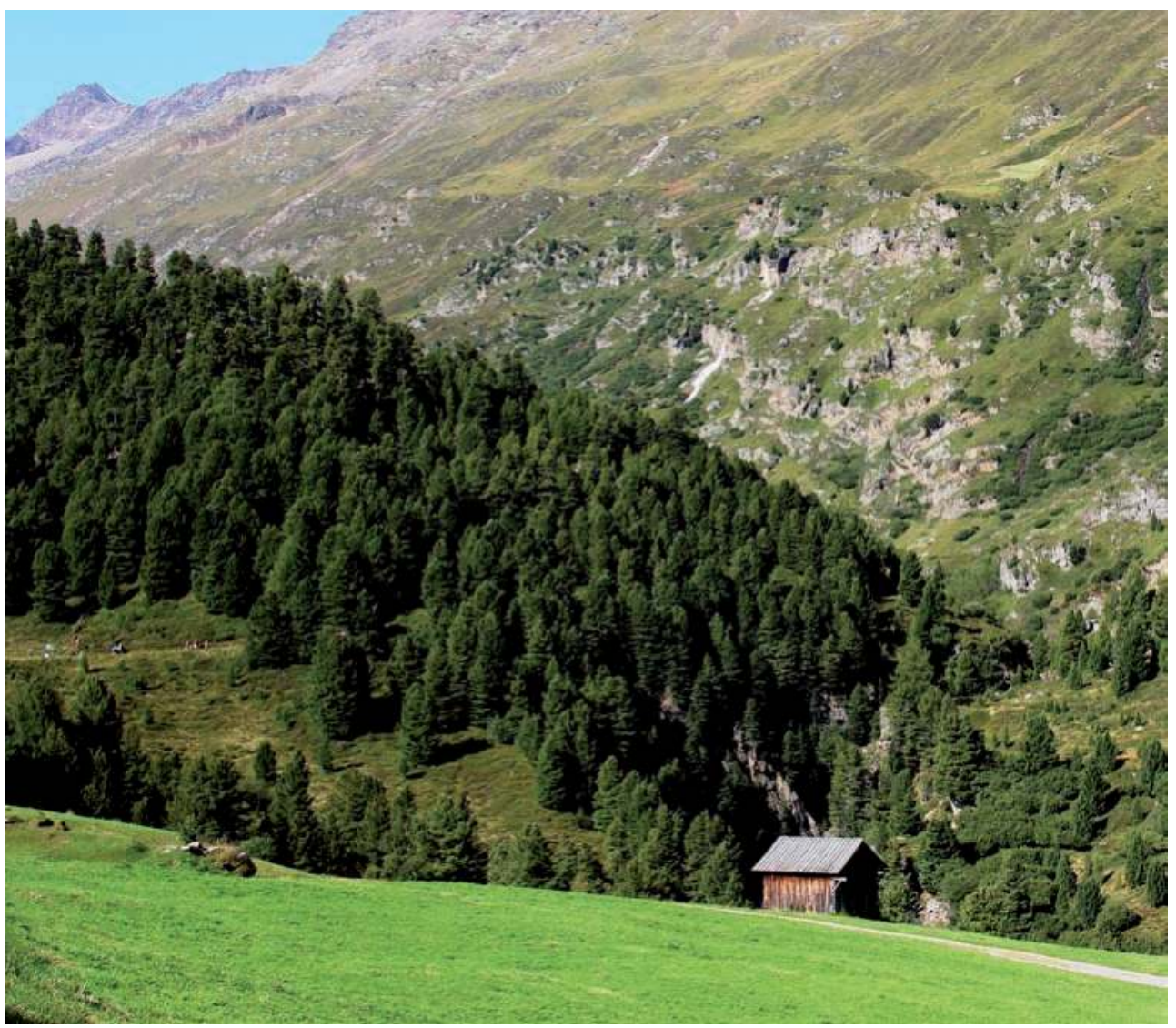

Abb. 4:

Blick auf den geschlossenen Zirbenwald südwestlich von Obergurgl. (Foto: Brigitta Erschbamer) 


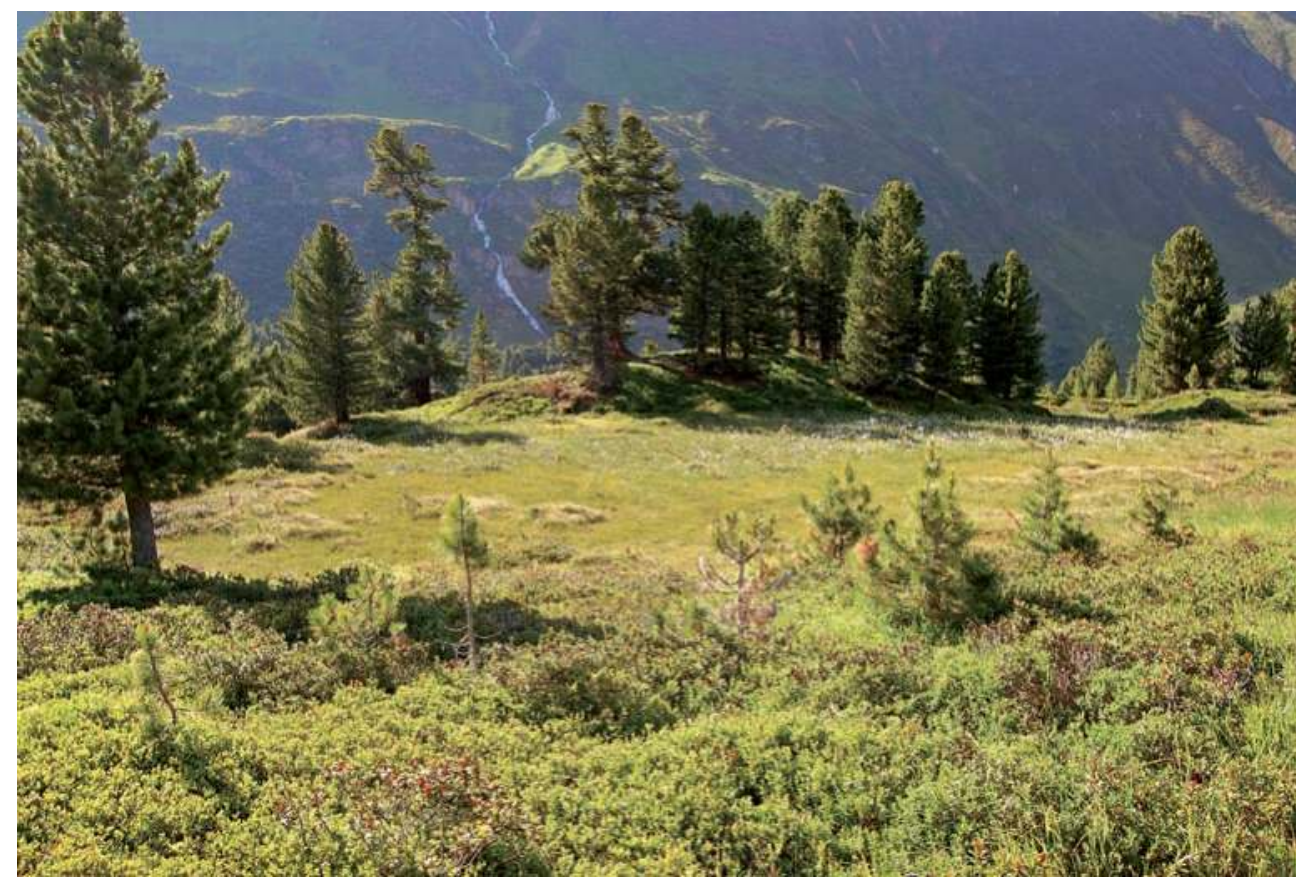

Abb. 5:

Oberhalb von ca. 2100 m Meereshöhe lockert der Obergurgler Zirbenwald immer mehr auf. Die Zirben stocken dann meistens auf Felsköpfen (Hintergrund) rund um die Moore, die die Senken und ebenen Flächen bestimmen. Hier wurde teilweise aufgeforstet. (Foto: Roland Mayer)

angeordnet (Abb. 1). Es wurden auch die Bestände an den steilen, felsigen, in nordwestliche Richtung exponierten Hängen mit einbezogen, nicht jedoch die Zirbenwaldmoore, welche von Rybníček \& Rybníčková (1977) erforscht worden sind. Auf der Gurgler Heide wurden sechs Zwergstrauchbestände an Windkanten und zehn in Hangmulden bzw. auf grobem Blockschutt zwischen 2150 und 2250 m Meereshöhe untersucht (Abb. 2). Die Größe der Aufnahmeflächen entsprach dem Minimumareal und betrug im Obergurgler Zirbenwald $200 \mathrm{~m}^{2}$, in den hochwüchsigen Zwergstrauchbeständen $50 \mathrm{~m}^{2}$ und in den niedrigwüchsigen Zwergstrauchheiden $25 \mathrm{~m}^{2}$.

\section{Frequenzanalysen und Datenverarbei- tung}

Die Dauerflächen im Bereich der Zwergstrauchheiden R, W und P wurden mit Hilfe eines Frequenzrahmens aufgenommen, wobei der Rahmen mittels Ösen exakt über den Bodenmarkierungen fixiert wurde. Der Rahmen wurde durch 
Bespannung in 100 Teilquadrate zu je 10 x $10 \mathrm{~cm}$ unterteilt. In jedem dieser Teilquadrate wurde eine Artenliste erstellt (Präsenz/Absenz-Daten). Die Frequenz einer Pflanzenart gibt an, in wie vielen der 100 Teilquadrate sie vorkommt (Dierschke 1994). Meist erfolgt die Angabe in Prozent. Die Frequenzaufnahmen in den Dauerflächen fanden in den Jahren 2000, 2003, 2004 und 2008 statt.

Für die Auswertung wurden die Arten nach Lebensformen (Zwergsträucher, Grasartige, Kräuter, Moose und Flechten) differenziert. Die Frequenzen der Lebensformen wurden aufsummiert und die Mittelwerte dieser Summen für jeden der drei Standorte berechnet. Außerdem wurden die Mittelwerte für die Artenzahl berechnet.

Die Nomenklatur der Gefäßpflanzen richtete sich nach Fischer et al. (2008), die der Moose nach Frahm \& Frey (2004), jene der Flechten nach Wirth (1995). Die Moose und Flechten wurden dankenswerterweise von Univ.-Prof. Dr. Georg Gärtner (Institut für Botanik, Universität Innsbruck) nachbestimmt.

\section{Pflanzensoziologische Auswertung}

Die Aufnahmen wurden mittels TWINSPAN (Hill 1979) klassifiziert. Zur besseren Vergleichbarkeit der Gesellschaften mit Literaturdaten wurden Stetigkeitstabellen erstellt. Die relative Stetigkeit einer bestimmten Pflanzenart ist ihr pro- zentuelles Vorkommen innerhalb einer gegebenen Anzahl von Aufnahmen. Es wurden folgende Stetigkeitsklassen unterschieden: $\mathrm{V}=>80 \%$, IV $=61-80 \%$, $\mathrm{III}=41-60 \%, \mathrm{II}=21-40 \%, \mathrm{I}=\leq 20 \%$ (Dierschke 1994).

\section{Ergebnisse}

\subsection{Obergurgler Zirbenwald}

In der Baumschicht des Obergurgler Zirbenwaldes herrscht Pinus cembra fast unumschränkt vor (Abb. 4). Abgesehen davon mischen sich nur einzelne Exemplare von Picea abies, Larix decidua und Sorbus aucuparia (Vogelbeere) dazu. Die Baumschicht ist überwiegend aufgelockert und weist eine Kronendeckung von durchschnittlich 30-50 \% auf. Stellenweise wird der Bestand aber auch dichter, wobei die Kronendeckung bis zu 80 \% erreicht. Die Strauchschicht ist meist spärlich ausgebildet und deckt nur in Einzelfällen maximal bis $30 \%$, lediglich in einer der Aufnahmen erlangte sie $60 \%$ Deckung. In der Strauchschicht herrscht der Jungwuchs von Pinus cembra vor. Unter den Sträuchern ist nur Lonicera caerulea (Blaue Heckenkirsche) häufig, welche in manchen der Aufnahmen Deckungswerte von $5 \%$ aufweist. Vereinzelt kommt der Jungwuchs von Picea abies, Larix decidua und Betula pendula (Hänge-Birke) auf. 


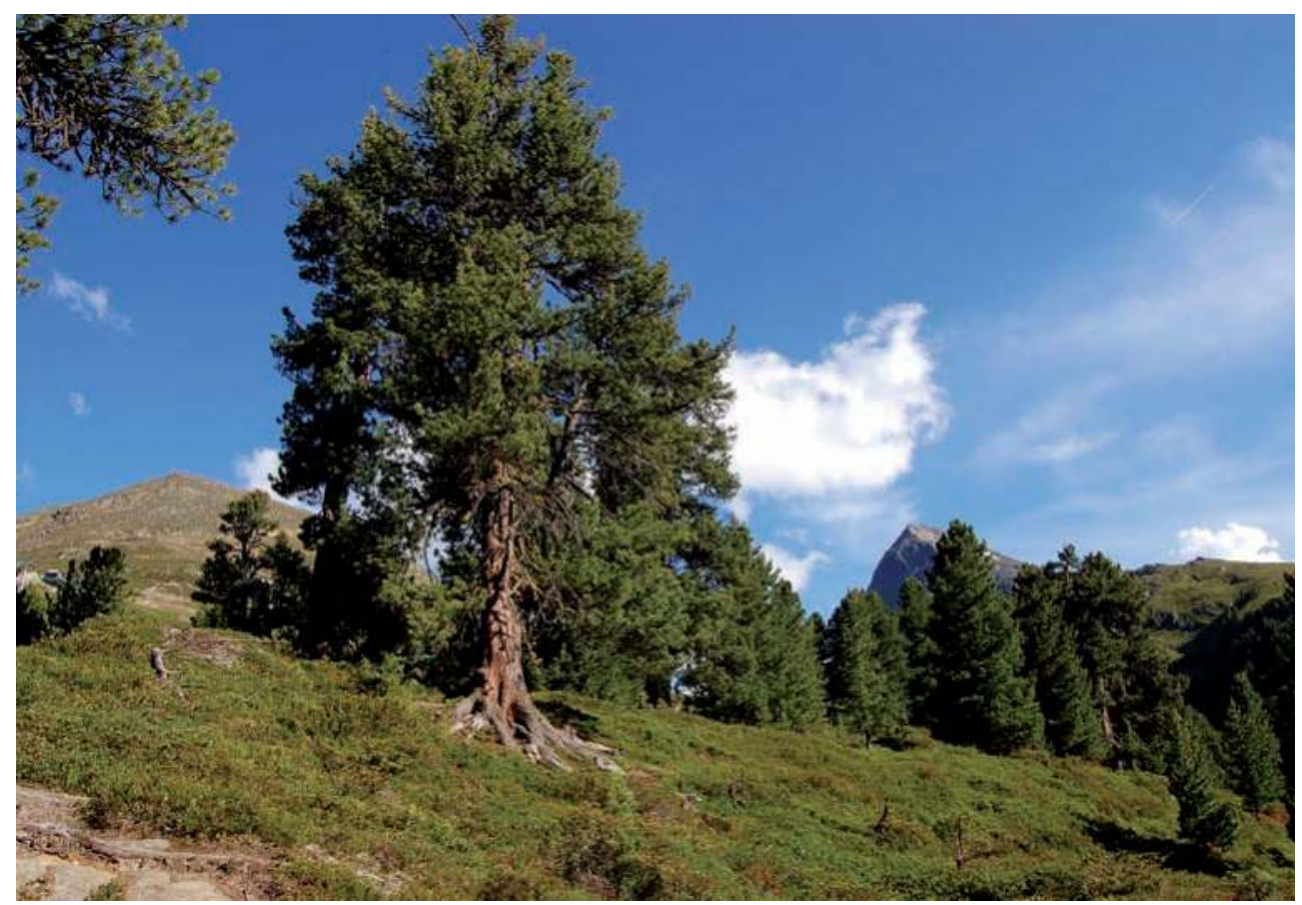

Abb. 6:

Der Obergurgler Zirbenwald ist ein einschichtiger Baumbestand mit alten Zirben (Hintergrund). Rhododendron ferrugineum (Rostrote Alpenrose; Vordergrund) wird vor allem an den offenen Flächen zwischen den Zirben dominant, dort, wo die Kronendeckung kaum mehr gegeben ist und wo damit eine sichere Schneedecke garantiert ist. (Foto: Roland Mayer)

Es gedeihen zudem einzelne Exemplare von Pinus mugo (Leg-Föhre, Latsche). Der Unterwuchs wird überwiegend von Zwergsträuchern (bis $80 \%$ Deckung) dominiert (Abb. 6), wobei Rhododendron ferrugineum am wichtigsten ist. Erst $\mathrm{ab}$ ca. $2100 \mathrm{~m}$ Meereshöhe geht die Vorherrschaft der Rostroten Alpenrose allmählich zurück. Rhododendron ferrugineum gedeiht bevorzugt in Mulden oder auf grobem Blockschutt an Bereichen ohne nennenswerte Kronendeckung und wird vorwiegend von Juniperus communis ssp. nana (Zwergwacholder) begleitet. Von den Zwergsträuchern der Gattung Vaccinium sind mit in etwa gleichen Anteilen $V$. vitis-idaea und V. gaultherioides vertreten, oft jedoch herrscht $V$. myrtillus vor. In den stärker aufgelichteten Beständen der höheren Lagen gedeiht auch Loiseleuria procumbens. Grasartige spielen im Unterwuchs eine wichtige Rolle, wobei sie vereinzelt bis zu $50 \%$ des Bodens bedecken. In manchen der Flächen ist ein dichter Grasfilz ausgebildet, welcher vor allem von Calamagrostis villosa (Wolliges Reitgras) 
aufgebaut wird. Mit sehr hoher Stetigkeit tritt Avenella flexuosa (Draht-Schmiele) auf. Sie deutet auf eine beträchtliche Versauerung des Bodens hin. Die Bedeutung der Grasartigen geht in höheren Lagen oberhalb von $2040 \mathrm{~m}$ Meereshöhe deutlich zurück. Es kommen zahlreiche Kräuter vor, ihre Deckung bleibt allerdings gering (meist nur zwischen 5 und $10 \%$ ). Im Obergurgler Zirbenwald gedeihen immer wieder Weidezeiger wie etwa Lotus corniculatus (Hornklee), Potentilla aurea (Gold-Fingerkraut) und Mutellina adonidifolia (Alpen-Mutterwurz). Hochstauden wie Gentiana punctata (Punktierter Enzian) treten zumeist in den Hintergrund. Eine durchwegs große Bedeutung erlangen Moose und Flechten. So ist die Moos- und Flechtenschicht meistens sehr gut ausgebildet und weist eine Deckung von bis zu $50 \%$ und in höheren Lagen bis $75 \%$ auf. Die wichtigsten Waldmoose sind Dicranum scoparium (BesenGabelzahnmoos), Pleurozium schreberi (Rotstängelmoos), Hylocomium splendens (Stockwerkmoos), Rhytidiadelphus triquetrus (Großes Kranzmoos) und Racomitrium sudeticum (Sudeten-Zackenmütze). Unter den Strauchflechten herrschen Cladonia rangiferina (Rentierflechte) und Cetraria islandica vor, von den Laubflechten ist Peltigera aphthosa (Apfelflechte) am auffälligsten. An den Stämmen und Ästen der Zirben gedeiht in oft üppigem Ausmaß Pseudevernia furfuracea (Baummoos) und vielfach auch die leuchtend gelbe $L e$ tharia vulpina (Wolfsflechte).
Pflanzensoziologisch lässt sich der Obergurgler Zirbenwald der Klasse VaccinioPiceetea Br.-Bl. in Br.-Bl. et al 1939 (Nordisch-alpische Nadelwälder, bodensaure Latschengebüsche und Birkenbruchwälder) und dort der Ordnung Junipero-Pinetalia mugo Boşcaiu 1971 (boreale Nadelwälder) zuordnen (Willner \& Grabherr 2007). Für die Zugehörigkeit zum Verband Pinion mugo Pawł. 1978 s.l. (subalpine Lärchen-, Zirben- und Bergföhrenwälder) sprechen die Verbandscharakterarten Pinus cembra (Baumschicht), Larix decidua (Baumschicht) und Pinus mugo (Strauchschicht) (Tab. A1, Spalten 1a, 1b). Die meisten der für die SilikatLärchen-Zirbenwälder typischen konstanten und dominanten Begleiter treten auch im Obergurgler Zirbenwald auf (Tab. A1, Spalten 1a, 1b). Der Obergurgler Zirbenwald gehört zur Assoziation Vaccinio-Pinetum cembrae (Pollmann \& Haffter 1933) Oberd. 1962 und kann aufgrund der verschiedenen Weidezeiger der Subassoziation nardetosum zugeordnet werden (Willner \& Grabherr 2007). Eine der Aufnahmen an den steilen Nordwesthängen der Gurgler Ache weist Bezüge zur Subassoziation alnetosum alnobetulae auf. Es lassen sich je nach Höhenlage zwei Ausbildungen unterscheiden, welche durch Differenzialartengruppen voneinander abgegrenzt werden können ( $D$ 1a, D 1b, Tab. A1). Die Ausbildung mit Oxalis acetosella (15 Aufnahmen) umfasst den Zirbenwaldbestand zwischen 1950 und $2040 \mathrm{~m}$ Meereshöhe, in welchem die 
Kronendeckung durchschnittlich $49 \%$, maximal bis zu $80 \%$ beträgt (Tab. A1, Spalte 1a). Die mittlere Artenzahl beläuft sich jedoch auf nur 27 Arten pro $200 \mathrm{~m}^{2}$. Die Ausbildung mit Loiseleuria procumbens (5 Aufnahmen) gedeiht in deutlich größerer Meereshöhe zwischen 2100 und 2160 m (Tab. A1, Spalte 1b). Sie weist eine niedrigere Kronendeckung von 10$60 \%$ (im Durchschnitt $32 \%$ ) auf, wobei aber die mittlere Artenzahl mit 47 Arten pro $200 \mathrm{~m}^{2}$ deutlich höher ausfällt (Abb. 5). Während Rhododendron ferrugineum in der Ausbildung mit Oxalis acetosella ihren Schwerpunkt hat, treten in der Ausbildung mit Loiseleuria procumbens vor allem Cladonia arbuscula, Hieracium glabratum (Kahlblatt-Habichtskraut) und Festuca halleri (Felsen-Schwingel) hervor. Diese Arten sind typisch für Windkanten- und Felsenrasen-Gesellschaften. Die Moos- und Flechtenschicht ist mit durchschnittlich $58 \%$ Deckung deutlich stärker vertreten als in der Ausbildung mit Oxalis acetosella mit im Mittel $26 \%$.

\subsection{Zwergstrauchheiden}

In Obergurgl wurden zwei Typen von Zwergstrauchheiden untersucht, welche sich durch ihre mittlere Wuchshöhe deutlich unterscheiden. Die hochwüchsigen Zwergstrauchheiden werden durchwegs von Rhododendron ferrugineum aufgebaut (Tab. A1, Spalte 2; Abb. 6). Weitere wichtige Zwergsträucher sind Juniperus communis ssp. nana, Empetrum hermaphroditum, Vaccinium myrtillus und Vaccinium gaultherioides. Nur vereinzelt kommt Arctostaphylos uva-ursi (Arznei-Bärentraube) vor, dann jedoch mit hoher Deckung. Die Zwergsträucher decken zwischen 65$85 \%$. Demgegenüber kommt den Grasartigen (5-15\% Deckung) und Kräutern (5-10\% Deckung) eine deutlich geringere Bedeutung zu. Die Deckung der Moos- und Flechtenschicht wechselt stark und beträgt zwischen 10 und $70 \%$. Unter den Gräsern herrschen Avenella flexuosa, Avenula versicolor (Bunt-Wiesenhafer) und Nardus stricta vor. Von den Kräutern fallen vor allem Arten auf, welche für beweidete Nardeten (Bürstlingsrasen) typisch sind, wie Potentilla aurea, Mutellina adonidifolia, Lotus corniculatus und Ranunculus montanus (Berg-Hahnenfuß). Diese hochwüchsigen Zwergstrauchheiden gedeihen an den in nordwestliche Richtung geneigten Hängen unmittelbar oberhalb des Dorfzentrums von Obergurgl in Hangmulden, oft auf blockigem Material. Hier herrscht Rhododendron ferrugineum deutlich vor. Im untersten Hangabschnitt werden die Zwergstrauchbestände von Grünerlengebüschen abgelöst. In aufgelockerten Grünerlengebüschen treten die Zwergsträucher überwiegend noch deckend in Erscheinung. Bei zunehmend geschlossener Deckung von Alnus alnobetula (Grünerle) verschwindet Rhododendron ferrugineum rasch (R. Mayer, unpubl. Daten). 


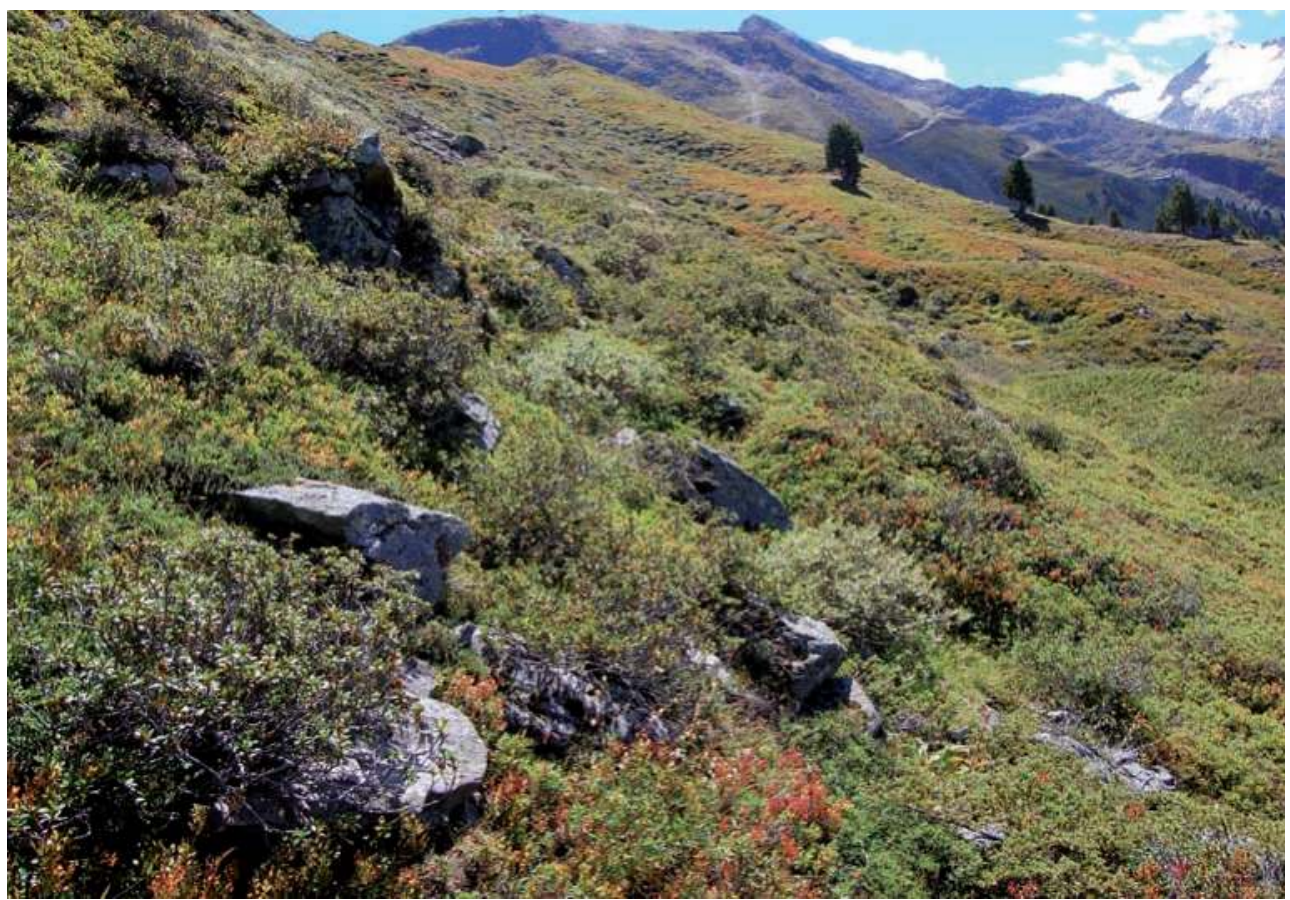

Abb. 7:

Blick auf einen hochwüchsigen Zwergstrauch-Bestand auf der Gurgler Heide, welcher dem Rhododendretum ferruginei zugeordnet wurde. Es herrscht Rhododendron ferrugineum vor (Vordergrund), doch auch Vaccinien prägen den Bestand (in herbstlicher Färbung). Das Rhododentretum ferruginei stockt bevorzugt auf grobem Blockmaterial. (Foto: Roland Mayer)

In höheren Lagen des Untersuchungsgebietes gedeiht im Anschluss an die in Mulden gelegene hochwüchsige Zwergstrauchheide ein niedrigwüchsiger Zwergstrauchbestand an Windkanten, der von den Teppichen des Spalierstrauches Loiseleuria procumbens geprägt wird (Abb. 7). Hier sind auch Strauchflechten der Gattungen Cetraria und Cladonia bedeutend, wobei an besonders exponierten und damit windgefegten Stellen Alectoria ochroleuca (Windbartflechte) stärker hervortritt (Tab. A1, Spalte 3). Die Zwerg- sträucher decken zwischen 55 und $85 \%$, auch die Moos- und Flechtenschicht (10$60 \%$ Deckung) ist sehr gut ausgebildet. Die Grasartigen treten demgegenüber mit 5-25 \% Deckung klar zurück. Kräutern kommt hinsichtlich ihrer Deckung (ca. 1 $\%)$ nur eine geringe Bedeutung zu.

Die untersuchten Zwergstrauchheiden im Bereich der Gurgler Heide gehören pflanzensoziologisch zur Klasse LoiseleurioVaccinietea Eggler 1952 (Zwergstrauchheiden der Arktis und boreal-nemoralen Gebirge) und hier in die Ordnung Rho- 


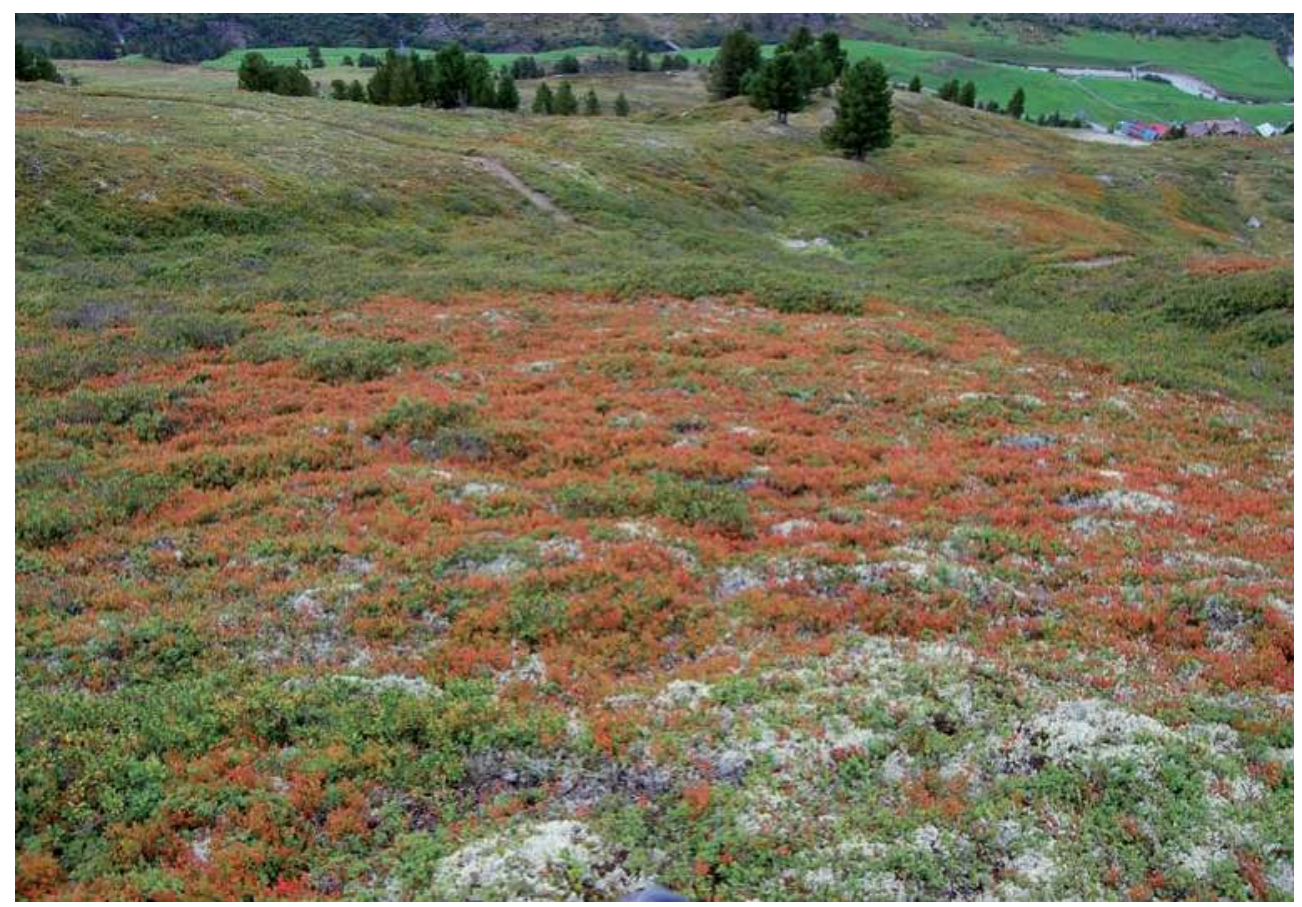

Abb. 8:

Die niedrigwüchsigen, spalierartigen Zwergstrauch-Bestände auf der Gurgler Heide werden von Flechten geprägt. Auffallend sind zudem die Vaccinien, welche durch ihre herbstliche Färbung hervorstechen. Im Hintergrund zu sehen sind einzelne Zirben, welche meist auf felsigen Kuppen gedeihen.

(Foto: Roland Mayer)

dodendro-Vaccinietalia Br.-Bl. in Br.-Bl. et Jenny 1926 (Azidophile Zwergstrauchheiden der Arktis und boreo-nemoraler Hochgebirge, Grabherr 1993). Die hochwüchsige Zwergstrauchgesellschaft gehört zum Verband Rhododendro-Vaccinion J. Br.-Bl. ex G. Br.-Bl. et J. Br.-Bl. 1931 (Bodensaure Alpenrosen- und Heidelbeerenheiden). Dafür ausschlaggebend ist die Dominanz der Charakterart Rhododendron ferrugineum (Tab. A1, Spalte 2). Die wichtigste Differenzialart gegenüber dem Loiseleurio-Vaccinion (Windheiden ne- moraler Hochgebirge) ist Juniperus communis ssp. nana, welche mit hoher Stetigkeit vorkommt. Gegenüber der niedrigwüchsigen Zwergstrauchgesellschaft ist das Rhododendretum ferruginei Rübel 1911 (Bodensaure Alpenrosenheide) durch eine umfangreiche Differenzialartengruppe deutlich unterschieden (D 2, Tab. A1). Als typische konstante und dominante Begleiter der Assoziation sind Vaccinium myrtillus, Avenella flexuosa, Pleurozium schreberi und Peltigera aphthosa zu nennen (Tab. A1, Spalte 2). 
Die niedrigwüchsige Zwergstrauchheide gehört zum Verband Loiseleurio-Vaccinion Br.-Bl. in Br.-Bl. et Jenny 1926 (Windheiden nemoraler Hochgebirge). Dafür sprechen die Charakterarten Loiseleuria procumbens und Empetrum hermaphroditum (Tab. A1, Spalte 3). Die Zugehörigkeit zur Assoziation Loiseleurio-Cetrarietum Br.-Bl. et al. 1939 (Alpenazaleen-Windheiden) ist durch die Charakterarten Hieracium alpinum (Alpen-Habichtskraut) und Alectoria ochroleuca belegt (Grabherr 1993). Zahlreiche dominante und konstante Begleiter wie etwa Cladonia arbuscula, Luzula lutea (Gold-Hainsimse), Vaccinium gaultherioides, Avenula versicolor und Phyteuma hemisphaericum (Grasblatt-Teufelskralle) sprechen für diese Zuordnung (Tab. A1, Spalte 3). Gegenüber dem Rhododendretum ferruginei hebt sich das LoiseleurioCetrarietum durch die hohe Stetigkeit der Flechten und die Präsenz von alpinen Arten ab (D 3, Tab. A1; Abb. 8). Es gedeiht auf der Gurgler Heide zwischen 2150 und 2220 m Meereshöhe an extremen Windkanten, die in nordwestliche Richtung geneigt sind. Loiseleuria procumbens und Vaccinium gaultherioides treten deckend hervor, während Empetrum hermaphroditum zwar eine hohe Stetigkeit aufweist, aber nur eine geringe Deckung zeigt (Tab. A1, Spalte 3). Carex curvula ist zwar hochstet, ihr Bestand ist jedoch sehr lückig. Juncus trifidus (Dreiblatt-Simse) kann vertreten sein.

\subsection{Veränderungen in den Dauerflächen der Zwergstrauchheiden}

Die stärksten Veränderungen hinsichtlich Artenzusammensetzung und -zahl wurden in der Zwergstrauchheide auf dem Rumsoppen $(2080 \mathrm{~m})$ beobachtet (Tab. 1): Alle Lebensformen nahmen zwischen den Jahren 2000 und 2008 zu. Die stärksten Zunahmen verzeichneten die Moose, gefolgt von den Kräutern und den Zwergsträuchern. Die Frequenzen der Grasartigen und Flechten nahmen hingegen nur wenig zu. Die Artenzahl stieg ebenfalls deutlich an.

Im Vergleich dazu veränderten sich die Frequenzen der Lebensformen in der Windheide (2300 m) kaum. Im Vergleich zum Jahr 2000 nahmen die Frequenzen der Zwergsträucher, Kräuter und Flechten leicht ab (Tab. 1). Die Grasartigen und Moose zeigten kaum Veränderungen. Die Artenzahl blieb weitgehend konstant. Die Pistenheide veränderte sich hingegen deutlich stärker. Die Frequenzen der Kräuter waren im Jahr 2008 im Vergleich zu 2000 deutlich höher (Tab. 1). Auch die Frequenzen der Grasartigen nahmen zu. Moose und Flechten veränderten sich kaum. Die Zwergsträucher hingegen verzeichneten einen leichten Rückgang. Die mittlere Artenzahl veränderte sich nur unwesentlich. 
Tab. 1:

Veränderungen der mittleren Artenzahl und der Frequenzen von 2000 bis $2008 \pm$ Standardfehler in den drei Zwergstrauchheiden (R: Rumsoppen: $n=6$; W: Windheide: $n=4$; P: Pistenheide: $n=3$ ).

\begin{tabular}{lcccccc}
\hline & \multicolumn{2}{c}{ Rumsoppen } & \multicolumn{2}{c}{ Windheide } & \multicolumn{2}{c}{ Pistenheide } \\
& 2000 & 2008 & 2000 & 2008 & 2000 & 2008 \\
\hline Artenzahl & $28 \pm 1$ & $32 \pm 2$ & $17 \pm 1$ & $17 \pm 3$ & $21 \pm 1$ & $23 \pm 4$ \\
$\begin{array}{l}\text { Frequenzen } \\
\text { Zwergsträucher }\end{array}$ & $176 \pm 14$ & $274 \pm 30$ & $183 \pm 23$ & $161 \pm 34$ & $190 \pm 44$ & $178 \pm 24$ \\
$\begin{array}{l}\text { Frequenzen } \\
\text { Grasartige }\end{array}$ & $136 \pm 12$ & $153 \pm 14$ & $27 \pm 14$ & $32 \pm 16$ & $132 \pm 17$ & $185 \pm 30$ \\
$\begin{array}{l}\text { Frequenzen } \\
\text { Kräuter }\end{array}$ & $117 \pm 16$ & $227 \pm 24$ & $66 \pm 53$ & $56 \pm 40$ & $199 \pm 33$ & $330 \pm 66$ \\
& & & & & & \\
Frequenzen Moose & $3 \pm 1$ & $54 \pm 11$ & $23 \pm 10$ & $25 \pm 13$ & $49 \pm 36$ & $57 \pm 33$ \\
$\begin{array}{l}\text { Frequenzen } \\
\text { Flechten }\end{array}$ & $1 \pm 1$ & $5 \pm 4$ & $298 \pm 31$ & $249 \pm 27$ & $118 \pm 54$ & $122 \pm 42$ \\
\hline
\end{tabular}

\section{Diskussion}

\subsection{Zirbenwald}

Auffällig am Obergurgler Zirbenwald sind der fast durchwegs einschichtige Aufbau des Bestandes und der sehr geringe Anteil an anderen Baumarten. Der Mengenanteil Larix decidua zu Pinus cembra hängt ganz wesentlich vom Alter des Waldes und vom menschlichen Einfluss ab. Ein nahezu monodominanter Bestand aus Pinus cembra wie in Obergurgl weist auf eine naturnahe Schlusswaldgesellschaft hin (Mayer 1986). Zudem setzt sich der Baumbestand aus überwiegend mehr als 300 Jahre alten Zirben zusammen. Diese besondere Struktur ist auf einen Waldbrand im Jahr 1880 zurückzuführen, bei welchem vor allem nur die alten Zirben überlebten (Landmann 2006).

Der Obergurgler Zirbenwald wurde der von Willner \& Grabherr (2007) ausgewiesenen Subassoziation Vaccinio-Pinetum cembrae nardetosum zugeordnet, d.h. einem Waldtyp mit typischen Weidezeigern. In der näheren Umgebung 
existieren zahlreiche Weideflächen und auch aktuell könnten sich durchaus noch vereinzelt Weidetiere im Zirbenwald aufhalten. In der Vergangenheit jedenfalls wurde der Obergurgler Zirbenwald intensiv beweidet. Insbesondere durch die Ziegenweide ist die Vegetation und vor allem die Zirbenverjüngung so stark in Mitleidenschaft gezogen worden, dass um ca. 1930 ein Weideverbot verhängt wurde (Neuwinger 1987). In den Ostalpen wurde historisch durch den Weidebetrieb im Waldgrenzbereich das Vaccinio-Pinetum cembrae (Silikat-Lärchen-Zirbenwald) durch das Rhododendretum ferruginei (Bodensaure Alpenrosenheide) vielfach ersetzt (Mayer 1974). Auch das Befahren mit Skiern im Winter stellt eine nicht zu unterschätzende Gefährdung für den Jungwuchs der Zirbe dar.

Klosterhuber (1994) untersuchte Lärchen-Zirbenwälder im inneren Ötztal bei Zwieselstein auf 1790-2060 m Meereshöhe. In diesen Beständen waren Larix decidua und Picea abies in der Baumschicht weitaus häufiger als im Obergurgler Zirbenwald. Unterluggauer (2003) belegte im benachbarten Vent das VaccinioPinetum cembrae durch vier Aufnahmen auf 1920-2110 m Meereshöhe. Im Vergleich zum Obergurgler Zirbenwald war Larix decidua in der Baumschicht stärker vertreten, Picea abies spielte auch hier keine nennenswerte Rolle. Auffällige floristische Ähnlichkeiten des Obergurgler Zirbenwaldes bestehen des Weiteren zum Rododendro ferruginei-Pinetum pros- tratae (Bodensaures Latschengebüsch) des Venter Tales, wobei es sich dort um Strauchbestände handelt, in welchen $\mathrm{Pi}$ nus cembra einen sehr lockeren Baumbestand bildet (Unterluggauer 2003).

Im Obergurgler Zirbenwald ist die Verjüngung von Pinus cembra - vor allem in den dichteren Beständen - als gering einzustufen. Es wurden nur vereinzelt Keimlinge von Pinus cembra gefunden. Junge Zirben kommen erst in den stärker aufgelichteten Beständen der höheren Lagen ab $2100 \mathrm{~m}$ besser auf. Das lässt sich dadurch erklären, dass die Zirbe eine Halbschattenbaumart ist, welche für den Aufwuchs ausreichend Licht benötigt (Tranquillini 1955). Außerdem dürfte die Verjüngung an den offenen Flächen in erster Linie auf den Tannenhäher (Nucifraga caryocatactes) zurückzuführen sein. Dieser Rabenvogel ist besonders dazu befähigt, Zirbenzapfen zu nutzen. Mit seinem starken Schnabel bearbeitet er sie effektiv und transportiert die Nüsschen in seinem Kehlsack. Er ist in der Lage, im weiten Umkreis zu ernten und durch sein ausgezeichnetes Ortsgedächtnis Vorräte anzulegen. Der Tannenhäher ist daher der häufigste und wichtigste Verbreiter der Zirbennüsse (Mattes 1982). Er legt auch gerne Verstecke oberhalb der Waldgrenze an. Zu den bevorzugten Verstecken gehören die Umgebung der Stammbasis unter dichten Kronen, felsige Kuppen sowie Abhänge und erhöhte Stellen wie etwa Zwergstrauch-Bulten und Baumstümpfe. In einem geringen Ausmaß werden auch 
offene Stellen wie Lichtungen genutzt. Die Verstecke im Boden legt der Tannenhäher immer in der Streu bzw. den obersten Bodenschichten an, nie in der Bodenvegetation (Mattes 1982). Mehr als $80 \%$ der Verstecke findet der Tannenhäher wieder, trotzdem bleibt meist ein beträchtlicher Überschuss an vergrabenen Zirbennüsschen übrig, welche sich zudem in einem günstigen Keimsubstrat befinden. Die Samenausbreitung durch den Tannenhäher ist besonders an der Waldgrenze und in degradierten Beständen von großer forstwirtschaftlicher Bedeutung (Mattes 1982). Verjüngungshemmend wirkt sich jedoch Calamagrostis villosa aus, ein ausläuferbildendes Gras, das einen dichten Filz bildet, in welchem es die Zirbenkeimlinge sehr schwer haben aufzukommen (Mayer 1974).

\subsection{Zwergstrauch-Gesellschaften}

Besonders die lokalen, mikroklimatischen Verhältnisse bestimmen zusammen mit dem Geländerelief die Verteilung der Zwergstrauchgesellschaften (Larcher 1977). Da in Obergurgl diesbezüglich keine detaillierten Untersuchungen vorliegen, dienen im Folgenden die Mikroklima-Untersuchungen vom Patscherkofel bei Innsbruck (1950 m Meereshöhe) als Grundlage für die Erläuterungen. Die wichtigsten klimatischen Faktoren sind Windgeschwindigkeit, Schneehöhe und Bodentemperatur. Es lässt sich ein Gradient beobachten, der von den hochwüchsigen RhododendronHeiden in schneereichen Lagen bis hin zur niedrigwüchsigen Loiseleuria-Heide in schneeärmeren oder schneefreien Lagen reicht. Rhododendron-Heiden sind im Winter durch die Schneedecke geschützt. Im Spätwinter, bei geringerer Dicke der Schneedecke, kann allerdings das Risiko für Frostschäden beträchtlich ansteigen, da Rhododendron ferrugineum nicht mehr ausreichend durch den Schnee geschützt wird (Neuner et al. 1999a,b). Die Frosttrocknis-Gefahr limitiert daher das Vorkommen von Rhododendron ferrugineum an schneearmen Standorten. Die Untersuchungen von Cernusca (1976) zeigten die mikroklimatischen Unterschiede der Zwergstrauchbestände sehr deutlich: während in einem Rhododendron-Bestand aufgrund der konstanten Schneedecke stabile Wintertemperaturen von ca. $0{ }^{\circ} \mathrm{C}$ herrschten, wies die schneefrei geblasene Loiseleuria-Heide zur selben Zeit extreme Erwärmung $\left(+30{ }^{\circ} \mathrm{C}\right)$ und Abkühlung $\left(-10^{\circ} \mathrm{C}\right)$ auf. Auch im Sommer unterschieden sich die Bestände markant: die Rhododendron-Heide blieb wesentlich kühler im Vergleich zur Loiseleuria-Heide. Der offene Aufbau der hochwüchsigen Rhododendron-Zwergstrauchbestände ermöglicht es, dass der Wind nahezu ungehindert durchziehen kann. Dadurch nehmen Temperatur und Luftfeuchtigkeit im Bestand merklich ab (Siegwolf \& Cernusca 1984). In der windexponierten Loiseleuria-Zwergstrauchheide hingegen 
ist das Mikroklima durch den spalierartigen, geschlossenen Wuchs der Gamsheide deutlich wärmer und feuchter (Cernusca 1976, Larcher 1977). Bestandesstruktur und Wuchshöhe üben damit einen entscheidenden Einfluss auf das Mikroklima aus (Körner 2003).

Die Aufnahmen auf der Gurgler Heide erfolgten im Bereich des potenziellen Waldgebietes. Im Rhododendretum ferruginei gedieh immer wieder der Jungwuchs der Zirbe, Keimlinge wurden hingegen nur selten gefunden. Der Jungwuchs der Zirben geht vermutlich auf Aufforstungstätigkeiten im Gebiet zurück. Im Loiseleurio-Cetrarietum traten häufiger Keimlinge von Pinus cembra auf, der Jungwuchs fehlte jedoch nahezu völlig. Das deutet darauf hin, dass sich im Loiseleurio-Cetrarietum Zirben-Keimlinge aufgrund der extremen Bedingungen nicht weiter entwickeln können und wieder absterben (Mattes 1982).

Im benachbarten Venter Tal untersuchte Unterluggauer (2003) ebenfalls Zwergstrauchheiden von 2000 bis 2500 $m$ Meereshöhe. Im Venter Tal ist ein artenreiches Junipero-Arctostaphyletum (Bärentrauben-Wacholderheide) ausgebildet. Es gedeiht auf steilen und auf sehr trockenen Sonnenhängen. Unterluggauer (2003) wies ein Empetro-Vaccinietum gaultherioidis (Krähenbeerenheide) aus, das durch die hohe Stetigkeit von Rhododendron ferrugineum Bezüge zum Rhododendretum ferruginei in Obergurgl erkennen lässt.

\subsection{Dynamik der Zwergstrauchheiden}

Die stärksten Veränderungen im Verlauf von neun Jahren wurden in der Zwergstrauchheide am Rumsoppen in 2040 m Meereshöhe festgestellt. Da sich in den Dauerflächen Weidezeiger wie Potentilla aurea, Ranunculus montanus und Lotus corniculatus finden, könnte es sich vielleicht um eine ehemalige Weide- oder Mahdfläche handeln, welche sich gerade im Verbrachungsstadium mit Zwergsträuchern befindet. Daten über die Beweidung in der Vergangenheit fehlen jedoch im Bereich dieser Zwergstrauchbestände. Eine Auflassung müsste dann allerdings schon vor sehr vielen Jahrzehnten erfolgt sein, denn Nagl (2009) beobachtete im Raum Obergurgl, dass selbst 50 Jahre alte Brachen, welche heute zwar optisch durch Zwergsträucher geprägt werden, eindeutig noch alle Elemente der subalpinen Wiesengesellschaften aufweisen. Abgesehen von einer abnehmenden Weidebelastung könnte auch eine Temperaturerhöhung im Zuge der Klimaerwärmung für die Kräuter von Vorteil gewesen sein. In der subalpinen Stufe stellt die Wärme einen limitierenden Faktor dar, daher sollte sich die Biomassenproduktion durch eine $\mathrm{Zu}$ nahme der Temperatur steigern. Denkbar ist auch, dass Stickstoffdepositionen zu einer deutlichen Zunahme der Frequenzen, vor allem der Kräuter, führten. Ähnliches gilt auch für die Pistenheide auf $2350 \mathrm{~m}$ Meereshöhe. Vermutlich wirkt sich vor allem der jährliche Düngereinfluss auf die 
Pisten aus. Die Auswirkungen der mechanischen Belastung durch den Skibetrieb müsste genauer untersucht werden. Der Boden wurde zwar weder planiert noch kommen Raupenfahrzeuge zum Einsatz, jedoch nutzen Skifahrer regelmäßig diesen Bereich (C. Kuen, persönl. Mitteilung). Neben einer Verdichtung des Schnees wirken sich vermutlich die Scherwirkungen durch Skikanten nachteilig auf die Vegetation aus (Grabherr 1987). Infolge dieser mechanischen Störungen werden die Winterknospen der Zwergsträucher beschädigt (Körner 2003). Die Kräuter haben vermutlich zusätzlich einen Vorteil erlangt, weil die Zwergsträucher durch das Befahren mit Skiern nur schlecht aufkommen (Wipf et al. 2005, Roux-Fouillet 2011). Im Vergleich zur Zwergstrauchheide am Rumsoppen und zur Pistenheide erwies sich die Windheide (Loiseleurio-Cetrarietum), abgesehen von jährlichen Fluktuationen, als stabil. Die extremen Verhältnisse, vor allem die sehr niedrigen Boden-Temperaturen im Winter, begünstigen wahrscheinlich die Flechten gegenüber den Gefäßpflanzen. Flechten wachsen jedoch sehr langsam, sodass sich die Frequenzen über die Jahre nur wenig änderten.

In Summe zeigte diese Studie, dass es in der subalpinen und unteren alpinen Stufe innerhalb von nur wenigen Jahren zu auffälligen Veränderungen in der $\mathrm{Zu}$ sammensetzung der Vegetation kommt. Ähnliches haben andere Autoren für die alpine und subnivale Stufe nachgewie- sen (Pauli et al. 2007, Erschbamer et al. 2009). Um zu klären, ob die festgestellten Veränderungen durch Klimaerwärmung, Stickstoffeintrag oder mechanische Belastungen infolge des Skibetriebs verursacht wurden, ist eine Fortsetzung des Monitorings notwendig, gekoppelt mit experimentellen Ansätzen zur Klärung der Einflussfaktoren.

\section{Literatur}

Aulitzky, H., Czell, A., Fromme, I., Neuwinger, I., Schiechtl, H.M. \& Stern, R. (1963) Beschreibung des Gurglertales. Barbeitet von H. Aulitzky u.a. Ökologische Untersuchungen in der subalpinen Stufe zum Zwecke der Hochlagenaufforstung. Mitteilungen der Forstlichen Bundes-Versuchsanstalt Mariabrunn 59.

Bortenschlager, S. (1993) Das höchst gelegene Moor der Ostalpen „Moor am Rofenberg" $2670 \mathrm{~m}$. Dissertationes Botanicae 196: 329-334.

Braun-Blanquet, J. (1964) Pflanzensoziologie. Grundzüge der Vegetationskunde. Springer Verlag, Wien, 865 S.

Casalegno, S., Amatulli, G., Camia, A., Nelson, A. \& Pekkarinen, A. (2010) Vulnerability of Pinus cembra L. in the Alps and the Carpathian mountains under present and future climates. Forest Ecology and Management 259: 750-761. 
Cernusca A. (1976) Bestandesstruktur, Bioklima und Energiehaushalt von alpinen Zwergstrauchbeständen. Oecologia Plantarum 11: 71-102.

Didier, L. (2001) Invasion patterns of European larch and Swiss stone pine in subalpine pastures in the French Alps. Forest Ecology and Management 145: 67-77.

Dierschke, H. (1994) Pflanzensoziologie. Grundlagen und Methoden. Verlag Eugen Ulmer, Stuttgart, $684 \mathrm{~S}$.

Ellenberg, H. \& Leuschner, C. (2010) Vegetation Mitteleuropas mit den Alpen in ökologischer, dynamischer und historischer Sicht. 6. Auflage. Ulmer, Stuttgart, 1333 S.

Erschbamer, B., Kiebacher, T., Mallaun, M. \& Unterluggauer, P. (2009) Short-term signals of climate change along an altitudinal gradient in the South Alps. Plant Ecology 202: 79-89.

Fischer, M.A., Oswald, K. \& Adler, W. (2008) Exkursionsflora für Österreich, Liechtenstein und Südtirol. 3. Auflage. Biologiezentrum der Oberösterreichischen Landesmuseen, Linz, 1394 S.

Frahm, J.-P. \& Frey, W. (2004) Moosflora. 4. Auflage. Ulmer UTB Stuttgart, 538 S.

Grabherr, G. (1987) Tourismusinduzierte Störungen, Belastbarkeit und Regenerationsfähigkeit der Vegetation in der alpinen Stufe. In: MaB-Projekt Obergurgl. Veröffentlichungen des Österreichischen MaB-Programms, Patzelt, G. (Hg.), Wagner Innsbruck 10: 243-256.

Grabherr, G. (1993) Loiseleurio-Vaccinietea. In: Die Pflanzengesellschaften Öster- reichs. Teil II: Natürliche waldfreie Vegetation, Grabherr, G. \& Mucina, L. (Hg.), 447-467.

Hill, M.O. (1979) TWINSPAN - a FORTRAN program for arranging multivariate data in an ordered two-way tableby classification of individualsandattributes. Cornell University, Ithaca, $90 \mathrm{~S}$.

Kaufmann, R. (2005) Langzeit-ÖkosystemMonitoring im Alpinen Raum. Endbericht 2000-2005. Innsbruck: Land Tirol, Abteilung Umweltschutz, 162 S.

Kilian, W., Müller, F. \& Starlinger, F. (1994) Die forstlichen Wuchsgebiete Österreichs. Eine Naturraumgliederung nach waldökologischen Gesichtspunkten. Forstliche Bundesversuchsanstalt, Wien, $60 \mathrm{~S}$.

Klosterhuber, R. (1994) Flora und Vegetation von Schutzwäldern der Tiroler Innenalpen. Diplomarbeit, Universität Innsbruck, $168 \mathrm{~S}$.

Klosterhuber, R. \& Hotter, M. (2001) Rote Liste der Wald- und Gebüschgesellschaften Nord- und Osttirols. Unveröffentlichter Bericht im Auftrag der Tiroler Landesregierung, Abteilung Umweltschutz.

Körner, C. (2003) Alpine Plant Life. Functional Plant Ecology of High Mountain Systems. $2^{\text {nd }}$ ed. Springer, Berlin, 344 S.

Krainer, K. (2010) Geologie und Geomorphologie von Obergurgl und Umgebung. In: Glaziale und periglaziale Lebensräume im Raum Obergurgl. Koch, E.-M. \& Erschbamer, B. (Hg.) innsbruck university press, Innsbruck: 31-52. 
Landmann, A. (2006) Alpiner Erlebnisweg Obergurgler Zirbenwald. Verein Naturpark Ötztal, $32 \mathrm{~S}$.

Larcher, W. (1977) Produktivität und Überlebensstrategien von Pflanzen und Pflanzenbeständen im Hochgebirge. Sitzungsbericht der Österreichischen Akademie der Wissenschaften, Mathematischnaturwissenschaftliche Kl., Abt. I, 186: 373-386.

Lumasegger, M., Hoffert, H., Auer, J. \& Angerer, H. (2009) Vegetationskartierung Ruhegebiet und Natura 2000-Gebiet Ötztal. Kartierung von Lebensraumtypen. Auftraggeber: Land Tirol, Abteilung Umweltschutz. REVITAL Ziviltechniker GmbH, Nußdorf-Debant, 76 S.

Mattes, H. (1982) Die Lebensgemeinschaft von Tannenhäher, Nucifraga caryocatactes (L.), und Arve, Pinus cembra L., und ihre forstliche Bedeutung in der oberen Gebirgswaldstufe. Eidgenössische Anstalt für das forstliche Versuchswesen, Birmensdorf 241, $74 \mathrm{~S}$

Mayer, H. (1974) Wälder des Ostalpenraumes. Gustav Fischer, Stuttgart, 346 S.

Mayer, H. (1986) Europäische Wälder. Gustav Fischer, Stuttgart, New-York, 386 S.

Moser, W. (1973) Naturdenkmal Obergurgler Zirbenwald, Jenny-Druck, Innsbruck.

Motta, R., Morales, M. \& Nola, P. (2006) Human land-use, forest dynamics and tree growth at the treeline in the western italian alps. Annals of Forest Science 63: 739-747.

Nagl, F. (2009) Vegetationskundliche Untersuchungen von Wiesen und Weiden im
Raum Obergurgl. Diplomarbeit, Universität Innsbruck, 129 S.

Neuner, G. (2007) Frost Resistance at the Upper Timberline. In: Trees at their Upper Limit. Treelife Limitation at the Alpine Timberline. Wieser, G., Tausz, M. (Hg.) Springer, Dordrecht: 171-180.

Neuner, G., Ambach, D. \& Aichner, K. (1999a) Impact of snow cover on photoinhibition and winter desiccation in evergreen Rhododendron ferrugineum leaves during subalpine winter. Tree Physiology 19: 725-732.

Neuner, G., Ambach, D. \& Buchner, O. (1999b) Readiness to frost harden during the dehardening period measured in situ in leaves of Rhododendron ferrugineum $\mathrm{L}$. at the alpine timberline. Flora 194: 289-296.

Neuwinger, I. (1987) Bodenökologische Untersuchungen im Gebiet Obergurgler Zirbenwald - Hohe Mut. In: Patzelt, G. (Hg.). MaB-Projekt Obergurgl. Veröffentlichungen des Österreichischen MaB-Programms, Wagner Innsbruck 10: 173-190.

Patzelt, G. (1997) Arbeiten aus dem Forschungsinstitut für Hochgebirgsforschung. Die Ötztalstudie - Entwicklung der Landnutzung. In: Alpine Vorzeit in Tirol. Begleitheft zur Ausstellung. Oeggl K., Patzelt, G., Schäfer D. (Hg.) Universität Innsbruck: 45-62.

Pauli, H., Gottfried, M., Reiter, K., Klettner, C. \& Grabherr, G. (2007) Signals of range expansions and contractions of vascular plants in the high Alps: observations 
(1994-2007) at the GLORIA*master site Schrankogel, Tyrol, Austria. Global Change Biology 13: 147-156.

Pitschmann, H., Reisigl, H., Schiechtl, H. \& Stern, R. (1980) Karte der aktuellen Vegetation von Tirol $1: 100.000$. VII. Teil: Blatt 10, Ötztaler Alpen Meran. Documents pour la Carte de la Végétation des Alpes 23: 47-68 + 1 Karte.

Purtscheller, F. (1978) Ötztaler und Stubaier Alpen. Sammlung Geologischer Führer, Band 53 (2. Auflage). Gebrüder Borntraeger, Berlin, Stuttgart, $128 \mathrm{~S}$.

Reichelt, G. \& Wilmanns, O. (1973) Vegetationsgeographie. Westermann, Braunschweig, $210 \mathrm{~S}$.

Reisigl, H. \& Keller, R. (1999) Lebensraum Bergwald: Alpenpflanzen in Bergwald, Baumgrenze und Zwergstrauchheide, 2. Auflage. Spektrum Akademischer Verlag, Gustav Fischer, Heidelberg, Berlin, $158 \mathrm{~S}$.

Roux-Fouillet, P., Wipf, S. \& Rixen, C. (2011) Long-term impacts of ski piste management on alpine vegetation and soils. Journal of Applied Ecology 48/4: 906-915.

Rybníček, K. \& Rybníčková, E. (1977) Mooruntersuchungen im oberen Gurgltal, Ötztaler Alpen. Folia Geobotanica \& Phytotaxonomica 12/3: 245-291.

Schiechtl, H.M. \& Stern, R. (1975) Die Zirbe (Pinus cembra L.) in den Ostalpen,
Ötztaler Alpen und westliche Stubaier Alpen. Angewandte Pflanzensoziologie Österreich, Band 22, Agrarverband Wien, 84 S.

Siegwolf, R. \& Cernusca, A. (1984) $\mathrm{CO}_{2}$ Gaswechsel von Rhododendron ferrugineum an der alpinen Waldgrenze. Verhandlungen der Gesellschaft für Ökologie 12: 111-122.

Tranquillini, W. (1955) Die Bedeutung des Lichtes und der Temperatur für die Kohlensäureassimilation von Pinus cembraJungwuchs am hochsubalpinen Standort. Planta, Berlin, 46: 154-178.

Unterluggauer, P. (2003) Die Vegetation in Vent und Rofen (Ötztal Tirol). Diplomarbeit Universität Innsbruck, 166 S.

Wallnöfer, S. (1993) Erico-Pinetea. In: Die Pflanzengesellschaften Österreichs. Teil III: Wälder und Gebüsche: Mucina, L., Grabherr, G. \& Wallnöfer, S. (Hg.) 244-282.

Willner, W. \& Grabherr G. (2007) Die Wälder und Gebüsche Österreichs. Ein Bestimmungswerk mit Tabellen, 2 Bände. Elsevier München, $302+290 \mathrm{~S}$.

Wipf, S., Rixen, C., Fischer, M., Schmid, B. \& Stoeckli, V. (2005) Effects of ski piste preparation on alpine vegetation. Journal of Applied Ecology 42: 306-316.

Wirth, V. (1995) Flechtenflora. 2. Auflage. Eugen Ulmer UTB Stuttgart, 664 S. 
Brigitta Erschbamer

Institut für Botanik

Universität Innsbruck

Sternwartestr. 15, 6020 Innsbruck

Brigitta.Erschbamer@uibk.ac.at
Roland Mayer

Institut für Botanik

Universität Innsbruck

Sternwartestr. 15, 6020 Innsbruck

Roland.Mayer@uibk.ac.at 


\title{
Kapitel 5 I Wald- und Waldgrenzenforschung in Obergurgl -
}

\author{
Vergangenheit und Zukunft
}

Robert Jandl, Andreas Schindlbacher, Silvio Schüler, Dieter Stöhr

\section{Zusammenfassung}

Der Versuchsstandort Poschach/Obergurgl ist seit mehr als fünf Jahrzehnten Gegenstand forstlicher Forschungen. Die Projekte nahmen ihren Anfang, als in der ersten Hälfte des 20. Jahrhunderts durch die anthropogen verursachten Waldverluste im Bereich der Waldgrenze die Schutzfunktion des Waldes dramatisch verringert wurde. Für die Durchführung von Hochlagenaufforstungen waren keine Referenzen aus vergleichbaren Regionen verfügbar, sodass in einem angewandten Forschungsprojekt die erforderlichen Maßnahmen entwickelt wurden. Das bleibende Ergebnis dieses Projektes ist das WindSchnee-Ökogramm, das Hilfestellungen bei der räumlichen und zeitlichen Planung von Hochlagenaufforstungen bietet. Später hat sich das Forschungsinteresse anderen Themen zugewandt. Vor allem die Kohlenstoffspeicherung im Boden sowie in der Biomasse von Wäldern und Waldersatzgesellschaften im Bereich der alpinen Waldgrenze ist heutzutage von großem Interesse, da sie die nationale $\mathrm{Bi}$ - lanz der Treibhausgasemissionen beeinflusst. Ein weiteres drängendes Thema ist die Entwicklung von Konzepten der adaptiven Waldbewirtschaftung, um die Stabilität der Wälder auch unter geänderten Klimabedingungen und im Hinblick auf demographische Verschiebungen langfristig sicherzustellen.

\section{Abstract}

The experimental site Poschach/Obergurgl is already investigated for more than five decades. Research started when the protection function of forests was diminished in the first half of the $20^{\text {th }}$ century due to earlier anthropogenic reductions of the forested area in the timberline ecotone. For afforestation projects in high elevation no suitable reference cases from comparable regions were available. Consequently the appropriate measurements were developed in a project of applied science. The lasting product of the project is the WindSnow Ecogramme supporting practical fore- 
stry in the spatial and temporal organization of afforestation projects in the timberline ecotone. Later research addressed other topics. Especially the sequestration of carbon in soils as well as in biomass of forests and forestreplacing plant communities is nowadays of relevance due to its bearing on the national budget of greenhouse gas emissions. Projects on the topic are ongoing. A pressing need concerns the development of concepts for adaptive forest management in order to ensure the stability of forests under changing climatic and demographic conditions.

Geschichtlicher Anlass für die forstliche Forschung

Siedlungen im Gebirge sind zahlreichen Naturgefahren wie Hochwässern und Muren im Sommer und Lawinen im Winter ausgesetzt. Wälder können die Gefahren wesentlich verringern, wenn sie dauerhaft einen geeigneten Bestandesaufbau aufweisen. In den inneralpinen Gebirgsregionen Österreichs war die wichtigste Funktion des Waldes der Schutz der landwirtschaftlichen Nutzfläche, während die Holzproduktion als sekundär erachtet wurde. Bereits aus der Zeit vor dem Mittelalter sind Beschreibungen über $\mathrm{Na}$ turkatastrophen überliefert und sehr früh wurde erkannt, dass gesetzliche Regelungen der Waldnutzung notwendig sind. Im 16. Jahrhundert wurde der Wald vom
Staat in Besitz genommen und die Bevölkerung hatte bescheidene Nutzungsrechte. Nach den napoleonischen Kriegen gab es in Tirol eine kurze Episode, in der die Besitzverhältnisse des Waldes unklar waren. In dieser Zeit trat eine gewaltige Übernutzung des Waldes ein. Die Schuldenlast der Bauern und die Aussichtslosigkeit der Intensivierung des Ackerbaus waren der Anlass für großflächige Rodungen (Schwendungen) zur Erhöhung der Viehbestände und der Vergrößerung der Alm- und Waldweideflächen auf Kosten der Wälder. Dazu kam Streunutzung in den verbliebenen Wäldern und der erhöhte Holzbedarf zur Errichtung der Almgebäude. Der Wald degradierte schleichend und die Schutzwirkung verringerte sich. Lawinen, Muren und Bodenerosion schädigten die Siedlungen und zerstörten die landwirtschaftliche Nutzfläche. Im Bereich der potentiellen Waldgrenze war die landwirtschaftliche Fläche teilweise mit Zwergsträuchern zugewachsen, sodass sowohl der land- als auch forstwirtschaftliche Ertrag verringert wurde. Am Ende des 19. Jahrhunderts wurde ernsthaft an die Aufgabe der Landwirtschaft in den betroffenen Regionen gedacht (Fromme 1957, Fliri 1973, Johann 1986 und 2007, Schreiber 1957).

Zur Sicherstellung der Schutzfunktion des Waldes zum allgemeinen Wohl trat 1852 das Reichsforstgesetz in Kraft. Bereits ab dem Jahr 1884 regelte das Wildbachverbauungsgesetz den Überschneidungsbereich zwischen Forstrecht und 
Wasserrecht und machte Raumordnungsmaßnahmen im Wirkungsbereich von Wildbächen und Lawinen durchsetzbar (Plattner 1983, Sauermoser 1988).

Die vielen Beobachtungen über den $\mathrm{Zu}$ sammenhang des Abflussverhaltens von Wildbächen und der Waldausstattung des Einzugsgebietes wurden im klassischen schweizerischen Vergleich des vollständig bewaldeten Sperbelgraben mit dem schütter bewaldeten Rappengraben erstmals wissenschaftlich rigoros belegt (Hegg et al. 2004). Die hydrologischen Messungen wurden 1903 begonnen und werden als Musterbeispiel ökologischer Langzeitforschung bis zum heutigen Tag fortgesetzt. Dem Schweizer Beispiel folgend sind weltweit eine Reihe von Einzugsgebietsstudien begonnen worden, in welchen der Effekt der Waldbehandlung und verschiedener Umwelteinflüsse auf das Abflussverhalten untersucht wird (Likens et al. 1995, Ellenberg et al. 1986, Matzner 2004). Auch in Obergurgl war die Regulation des Abfluss-Geschehens ein zentrales Thema, wobei auf Erfahrungen aus anderen Tiroler Tälern zurückgegriffen wurde (Stauder 1963).

Zwischen den beiden Weltkriegen war die wirtschaftliche Situation der Forstwirtschaft in den Gebirgsregionen unerfreulich. Sogar im Wirtschaftswald waren die Aufforstungsrückstände erheblich und es fehlten die Mittel für Maßnahmen im Gebirgswald (Schreiber 1957). Die beiden katastrophalen Lawinenwinter 1951 und 1954 mit zahlreichen Todesopfern und wirtschaftlichen Verlusten, insbesondere in den inneralpinen Tälern, haben den Anlass für verstärkte Bemühungen um die Hochlagenaufforstungen gegeben. Die Hochlagenaufforstungsprojekte waren anfangs mit Mitteln des European Recovery Fund (ERP) kofinanziert. Die Projekte wurden als Integralmelioriation bezeichnet, da neben der eigentlichen Aufforstung auch die Landbewirtschaftung der Gefahrensituation angepasst wurde, indem die Waldweide möglichst abgelöst wurde, um die Viehwirtschaft auf den am besten geeigneten Standorten zu konzentrieren (Stauder 1968). Die ersten Hochlagenaufforstungen zeigten teilweise nur bescheidene Erfolge. Besonders flächendeckende Aufforstungen erwiesen sich als problematisch, da viele Pflanzen auf ungünstigen Kleinstandorten wie schneereichen Mulden und windexponierten Geländekanten eingebracht wurden, auf welchen ein erfolgreiches Aufkommen von vornherein nicht zu erwarten war und wo in der Tat die Ausfälle durch Pilzbefall, zum Beispiel bei der Zirbe mit Scleroderris lagerbergii, und abiotische Schäden beträchtlich waren. Zudem ist auf den geneigten Hängen die Zugbelastung durch die kriechende Schneedecke eine Gefahr für die Bäumchen, der aber mit technischen Schutzmaßnahmen begegnet werden kann (Markart et al. 2002, Sauermoser 1988).

Ebenso als problematisch erwies sich die Beschaffung von geeignetem Pflanzenmaterial, da Hochlagenherkünfte mit 
der notwendigen Angepasstheit an die widrigen Bedingungen nur selten fruktifizieren und eine geringere Keimfähigkeit sowie geringere Samengewichte aufweisen (Müller \& Schultze 1996). In diversen Projekten wurden daher verschiedene heimische und fremdländische Baumarten getestet, sowie vegetativ vermehrte Pflanzen aus den unterschiedlichsten Provenienzen angebaut (Stern \& Zwerger 1980, Weißenbacher et al. 2007 und 2011). Es wurde danach getrachtet, die Projekte auf einer soliden wissenschaftlichen Basis durchzuführen, um die Kleinstandorte identifizieren zu können, auf welchen eine Etablierung von Wald möglich wäre. Dazu wurde an der damaligen Forstlichen Bundesversuchsanstalt eine Arbeitsgruppe für die subalpine Waldforschung gegründet. Diese hat bald Weltruf erlangt und war die Grundlage für die internationale Bedeutung der österreichischen Baumphysiologie, die auch in vielen Publikationen deutlich wird (Tranquillini 1979, Larcher 2001, Körner 2003a, Wieser \& Tausz 2007).

\section{Hochlagen-Aufforstungsprojekt Poschach/Obergurgl}

Der Versuchsstandort Poschach/Obergurgl repräsentierte die Situation einer Siedlung an der oberen Höhengrenze und die Herausforderung für die Hoch- lagenaufforstung zur Wiederherstellung der Schutzwirkung des Waldes. Im Ötztal wurde die Waldfläche zwischen 1774 und 1880 um mehr als 50\% reduziert. Danach war bis zum Jahr 1953 noch eine leichte Entwaldungstendenz zu beobachten. Einzelgruppen von vitalen Zirben in 2400 m Höhe zeigten die mögliche Ausdehnung des Waldes. Die Landwirtschaft im Gurgler Tal brachte bescheidene Erträge der Hauptfrüchte Kartoffel, Rüben und Kohl. Die Tragfähigkeit der Region ,Oberes Ötztal' wurde in der Mitte des 19. Jahrhunderts mit 120 Personen beziffert und diese Anzahl wurde unter anderem durch eine restriktive Heiratspolitik strikt eingehalten (Fromme 1957, Moser \& Peterson 1981). Die Landnutzung wurde von alpinem Grasland und von Bergmähdern dominiert. Der Hochwald, bestehend aus Lärchen und Zirben, und die Zwergstrauchheiden nahmen weniger Fläche ein und wurden zudem beweidet (Abb. 1). Das Gebiet der Gemeinde Sölden ist derzeit zu 8\% bewaldet, wobei Lärchen-Zirbenwälder (Larici-Pinetum cembrae) dominieren (Kilian et al. 1994, Erschbamer 2007) und in Talnähe auch Fichte (Picea abies) und Weißkiefer (Pinus sylvestris) vorkommen (Tab. 1).

Der Ortsteil Poschach wurde 1951 von einer Lawine beschädigt, deren Abrissgebiet zwischen der aktuellen und der potentiellen Waldgrenze in etwa $2300 \mathrm{~m}$ Seehöhe lag (Fromme 1957). Es lag auf der Hand, dass Maßnahmen zum Schutz vor Naturgefahren erforderlich waren. Angesichts 


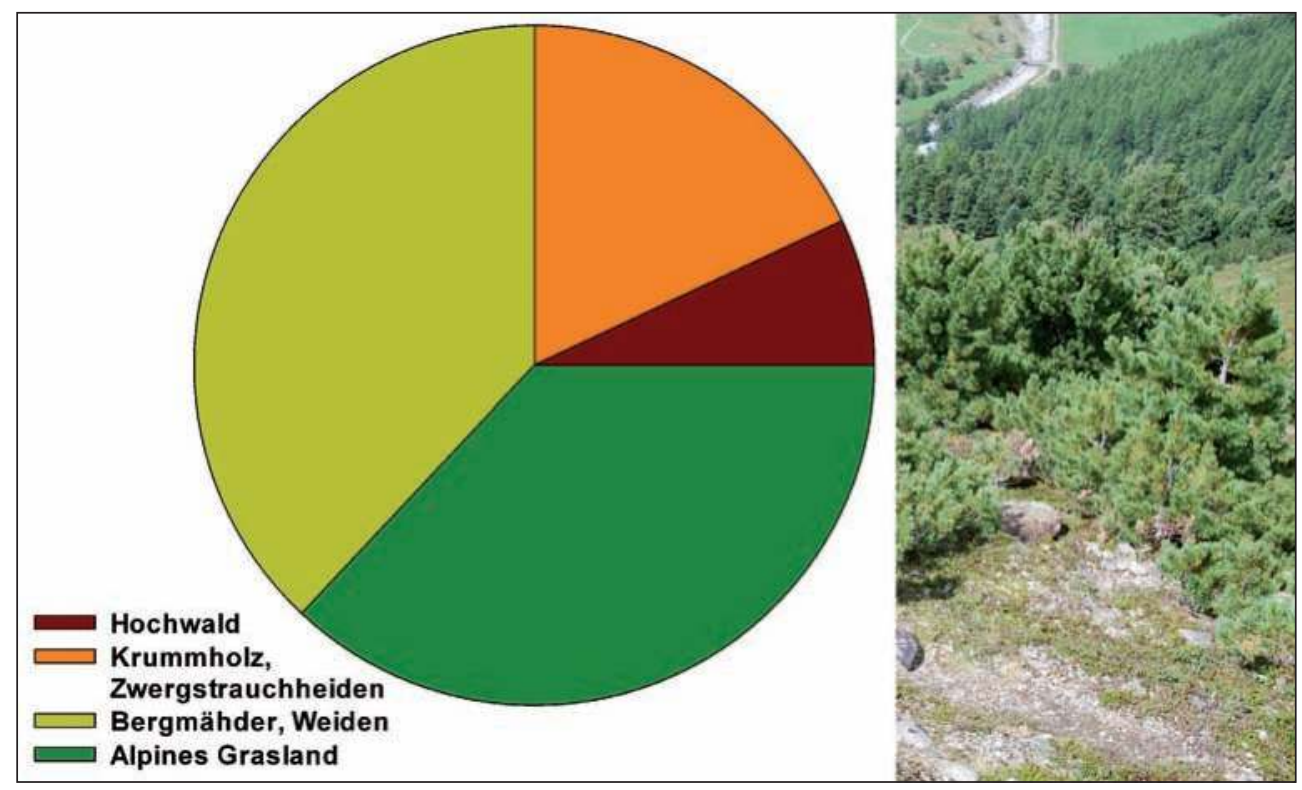

Abb. 1:

Anteile verschiedener Formen der Landnutzung im Gurgler Tal in den frühen 1950er Jahren.

(Quelle: Friedel 1961)

Tab. 1:

Die Waldausstattung der Gemeinde Sölden nach Angaben der Walddatenbank Tirol (ADTLR 2011)

\begin{tabular}{|l|c|l|c|}
\hline & Fläche [ha] & Besitzverhältnisse & [\% der Waldfläche] \\
\hline Hochwald & 3212 & Agrargemeinschaften & 74 \\
\hline Krummholz & 488 & $\begin{array}{l}\text { Gemeindewald und } \\
\text { offentlich-rechtlicher Wald }\end{array}$ & 9 \\
\hline & & Privatwald & 17 \\
\hline
\end{tabular}

der hohen Kosten für die Errichtung und Erhaltung von Lawinenschutzbauten im Abrissgebiet der Lawinen wurde entschieden, ein Hochlagenaufforstungsprojekt mit Mitteln des ERP Funds unter
Federführung der Forstlichen BundesVersuchsanstalt durchzuführen. Das Forschungsprojekt Poschach war ein Musterbeispiel für angewandte Forschung. Die konkrete Frage war, wie in der extrem 
schwierigen Lage ein stabiler Waldbestand etabliert werden kann. Der Erfahrungsschatz aus Voruntersuchungen war gering, sodass ein komplexer Geländeversuch eingerichtet wurde.

Der Lärchen-Zirbenwald reichte bis in eine Seehöhe von 2050 m. Das Versuchsgebiet reichte bis ans obere Ende der Beerrinne, die in $2220 \mathrm{~m}$ Seehöhe in eine Verebnung übergeht. Die Zone oberhalb der aktuellen Waldgrenze wurde als ,Kampfzone des Waldes' bezeichnet. Im Gebiet wurden umfangreiche Kartierungen der Vegetation, des Bodens, der Geländemorphologie gemacht und einige Parameter (z.B. Lufttemperatur, Luftfeuchte, Niederschlag, Windrichtung und -stärke, Bodentemperatur, Bodenfeuchte und Wasserverfügbarkeit, Schneehöhen und Ausaperung) wurden in zwei Bänden publiziert (Forstl. Bundesversuchsanstalt 1961 und 1963). Das Messprogramm war damals wissenschaftliches Neuland. Die Bedeutung der Bodentemperatur für die Keimbetteigenschaften der Jungpflanzen und die mikrobiologische Aktivität wurden erstmals für subalpine Standorte beschrieben und der Zusammenhang zwischen Vegetation und der Schneebedeckung wurde explizit dargestellt (Tab. 2). Außerdem wurde gezeigt, dass die Vegetationsperiode auf den Standorten nur 70 bis 90 Tage lang ist, in der Tat eine sehr kurze Zeit, um die Entwicklung abzuschließen und frosthart in den nächsten Winter zu kommen (Aulitzky 1961, Friedel 1961, Turner 1961).

Das am deutlichsten herausragende Ergebnis ist das ,Wind-Schnee-Ökogramm', in dem der Geländebefund zur Handlungsempfehlung für die Aufforstung verdichtet wurde (Abb. 2). Dabei wird aus der Artenkombination der krautigen Vegetation auf die Schneehöhe und

Tab. 2:

Zusammenhang zwischen Vegetationsform und Höhe der Schneedecke, die Hinweise auf die Maßnahmen zur Aufforstung geben

\begin{tabular}{|c|c|c|}
\hline $\begin{array}{l}\text { Höhe der } \\
\text { Schneedecke } \\
\text { im April [cm] }\end{array}$ & Pflanzengesellschaft & \\
\hline $5-10$ & Alectorietum ochroleucae & Windflechtenheide \\
\hline $10-30$ & Loiseleurietum procumbentis & Gemsheide \\
\hline $30-80$ & Vaccinetum uliginosi & Rauschbeerheide \\
\hline $80-200$ & $\begin{array}{l}\text { Rhododendretum ferruginei }+ \\
\text { Vaccinetum myrtilli }\end{array}$ & $\begin{array}{l}\text { Rostrote Alpenrose und } \\
\text { Heidelbeere }\end{array}$ \\
\hline
\end{tabular}




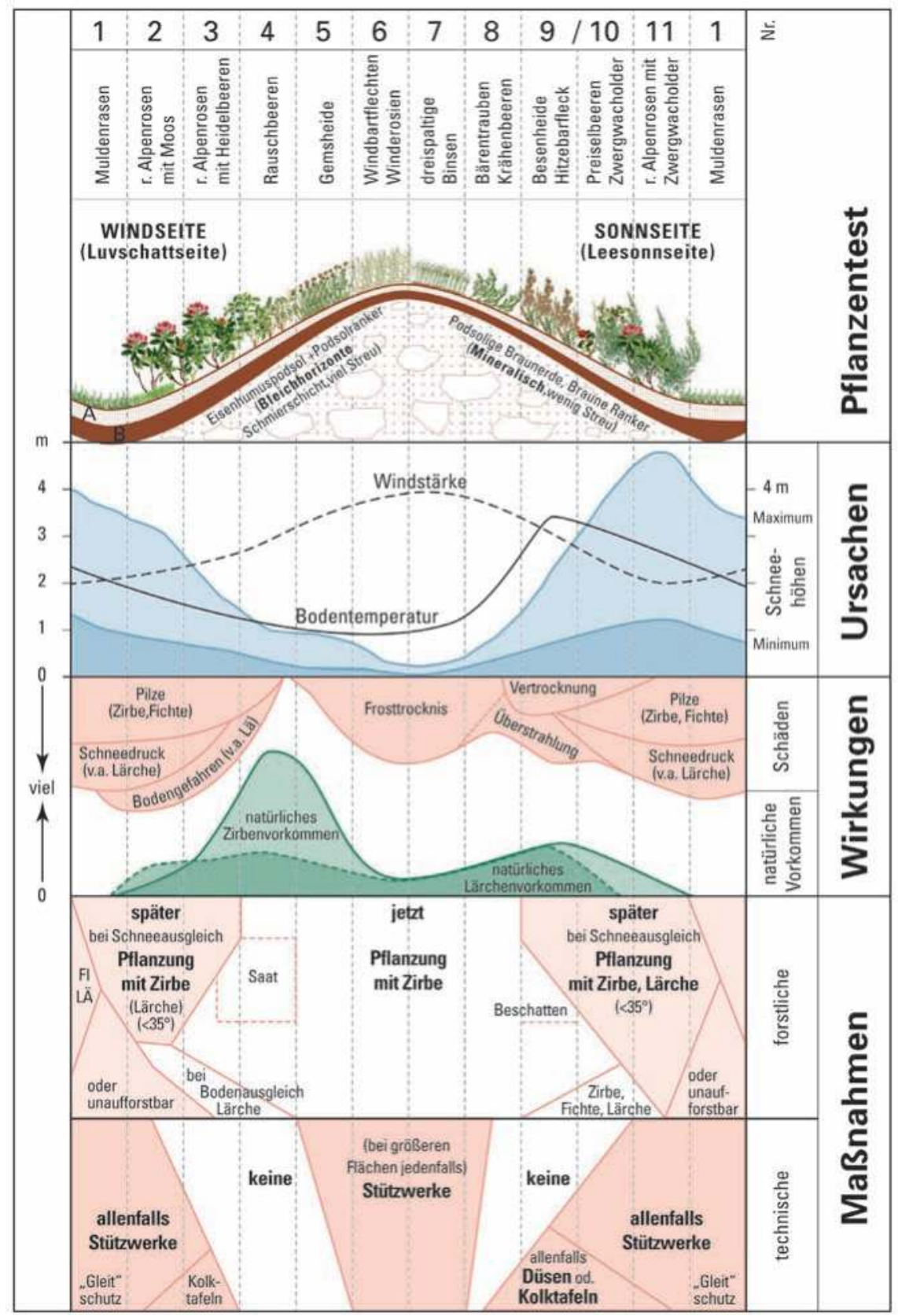

Abb. 2:

Vorläufiges Wind-Schnee-Ökogramm als Hilfsmittel für die Hochlagenaufforstung in den österreichischen Innenalpen (Aulitzky 1963). 
die Bodentemperatur geschlossen. Daraus lässt sich die Ursache von Schäden eruieren und zuletzt ist es möglich, die Hochlagenaufforstung mit forsttechnischen Maßnahmen zu unterstützen. Ein Zirben-Aufforstungsprojekt ist zeitlich am besten im späten Frühjahr durchzuführen. Räumlich beginnt es auf den Rippen eines Hanges, also dort, wo die natürliche Ausbreitung der Zirbe auch aktuell am deutlichsten stattfindet. Wenn eine Gruppe von Bäumchen der Rottenstruktur nach Mayer (1976), der schwierigsten Entwicklungsphase, entwachsen ist, verändert sie den Kleinstandort derartig, dass die Aufforstung um die entstandene Baumgruppe fortgesetzt werden kann und in Folge der bereits vorhandene Wald das Aufkommen weiterer Jungpflanzen begünstigt. In den schneereichen Mulden eines Hanges sind zusätzliche Schutzmaßnahmen erforderlich, um die Pflanzen vor Schneegleiten zu schützen (Aulitzky 1963 und 1992).
Der Erfolg des Hochlagenaufforstungsprojektes Poschach wird in Abb. 3 ersichtlich. Rezent reicht der Wald viel höher hinauf als in der Vergangenheit. Die antreibenden Faktoren dafür sind vielfältig und werden unter dem Sammelbegriff ,globale Veränderung' zusammengefasst. Dieser beschreibt den demographischen Wandel, der durch die Änderung der beruflichen Orientierung weg von Arbeitsplätzen in der Landwirtschaft zur Aufgabe der hochgelegenen Bergmähder und Weideflächen führt, andererseits ist der landwirtschaftliche Ertrag dieser Flächen aus betriebswirtschaftlichen Überlegungen von untergeordneter Bedeutung. Ganz entscheidend wird der Anstieg der Waldgrenze und die Wiederbewaldung der Zwergstrauchheiden vom Klimawandel begünstigt.

Die Projektlinie der Hochlagenaufforstungen wurde anfangs der 1970er Jahre in Obergurgl aufgegeben und nach Haggen

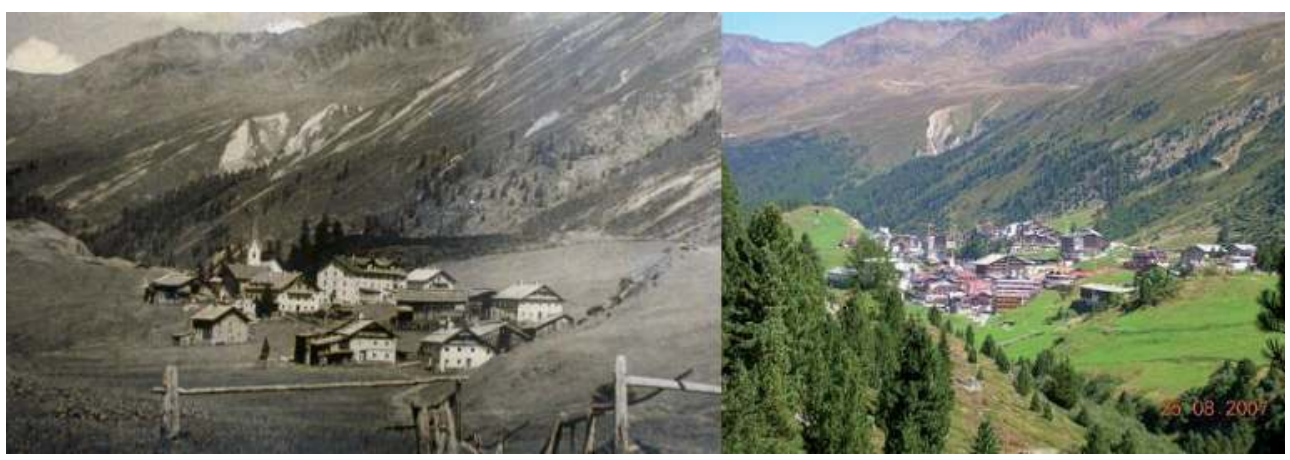

Abb. 3:

Ansicht der Ortschaft Obergurgl im Jahr 1920 (links) und 2007 (rechts). Im Hintergrund oberhalb der Kirchturmspitze ist die höhere Ausdehnung des Waldes in der Gegenwart zu erkennen. (Foto 1920: Alpine Forschungsstelle Obergurgl, Foto 2007: Robert Jandl) 
im Sellrain und zu anderen Standorten verlegt. In Haggen werden gemeinsam mit der Wildbachverbauungsbehörde praxisnahe Versuche durchgeführt. Dort wurde auch erstmals der Wasserhaushalt einer Hochlagen-Aufforstungsfläche im Vergleich mit der benachbarten Zwergstrauchheide untersucht (Kronfuss 1983, Markart 2000).

Ökosystemleistungen des Waldes Kohlenstoffsequestrierung

Der Begriff, Ökosystemleistungen des Waldes ${ }^{`}$ wird weit gefasst. Neben der tra-

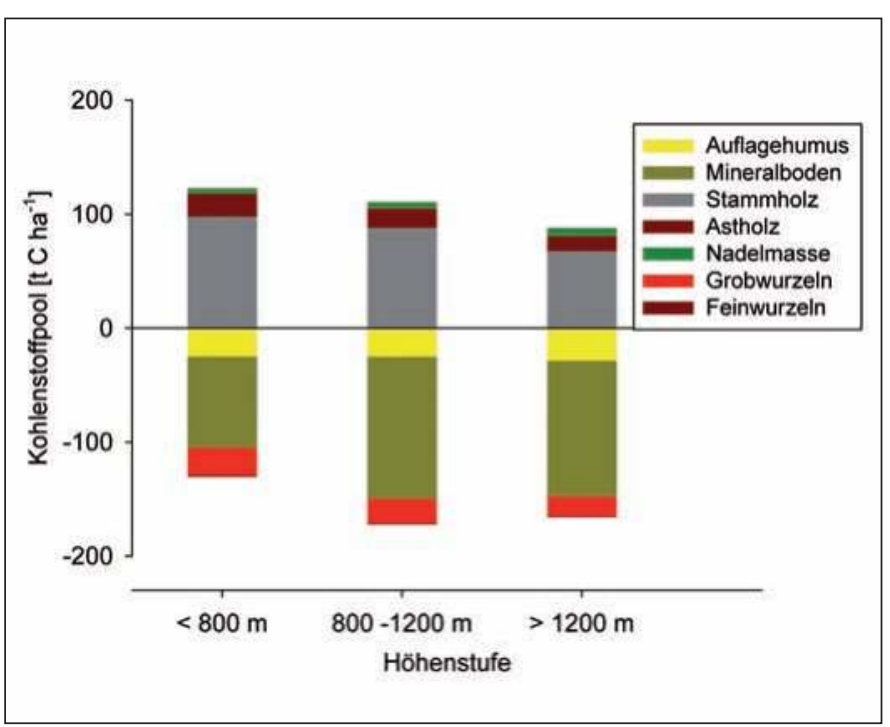

ditionellen Schutzfunktion des Waldes werden der Beitrag zur Festlegung von Kohlenstoff in der Biomasse und im Waldboden, die Bedeutung für die Bereitstellung von hochwertigem Wasser, die Biodiversität und die landschaftliche Schönheit als Werte von Waldökosystemen genannt. Diese Werte sind ökonomisch bewertbar, aber nur teilweise marktfähig (Naidoo et al. 2008, Schröter et al. 2005, Spangenberger \& Settele 2010, Thuiller et al. 2005). Die Hoffnungen der Waldbesitzer auf ein lukratives Nebenprodukt der forstlichen Bewirtschaftung haben sich bisher nicht ganz erfüllt. Die Ökosystemleistung, Festlegung von Kohlenstoff ist marktnahe, da durch die Anrechenbarkeit terrestrischer Kohlenstoffsenken in der österreichischen Treibhausbilanz eine Valorisierung möglich ist. Anhand von Abb. 4 wird deutlich, dass in Hochlagen dem Kohlenstoffspeicher im Waldboden eine große Bedeutung zukommt. Hier ist mehr Kohlenstoff gebunden als in der oberirdischen Biomasse, da die Produktivität der Gebirgswälder gering ist und andererseits durch die

Abb. 4:

Kohlenstoffvorrat in den österreichischen Wäldern. Datenquellen: Österreichische Waldinventur und Österreiche Waldbodenzustandsinventur; Projekte des Bundesforschungs- und Ausbildungszentrums für Wald, Naturgefahren und Landschaft (BFW); basierend auf einer Stichprobe von 140 Waldbeständen 


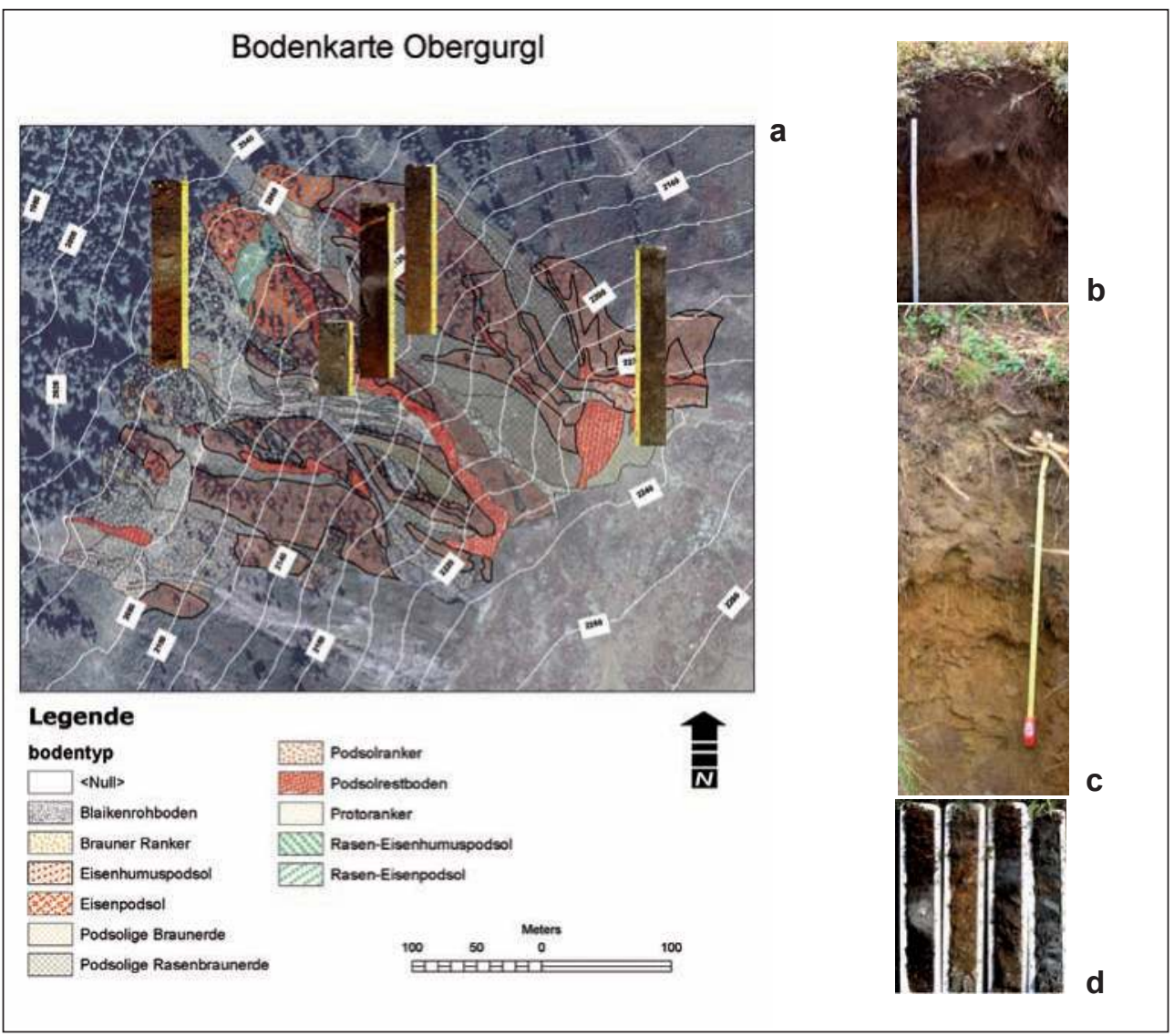

Abb. 5:

Die Verteilung von Bodentypen im Versuchsgebiet Poschach/Obergurgl mit einigen Leitprofilen (a), ein charakteristisches Bodenprofil des alten Zirbenwaldes (b), der Zwergstrauchheide (c) und ein Eindruck der kleinsträumigen Variabilität von Bodentypen (d)

Kürze der Vegetationsperiode und die niedrigen Temperaturen die Bedingungen für die Mikroorganismen des Bodens so ungünstig sind, dass Bodenkohlenstoff eher konserviert als abgebaut wird.

Im Projektgebiet Poschach war bei Geländearbeiten der Eindruck entstanden, dass die Böden des alten Zirbenwaldes weniger Kohlenstoff enthalten als die Bö- den der höher gelegenen Zwergstrauchheiden. Der quantitative Nachweis ist aufgrund der kleinräumigen Variabilität aufwendig (Abb. 5). Der Geländebefund widerspricht allerdings der gängigen Auffassung, dass Waldböden aufgrund der kontinuierlichen und erheblichen Dotierung mit organischer Substanz durch den ober- und unterirdischen Streufall reicher 
an organischer Substanz sind als Böden mit anderer Vegetationsbedeckung und Bewirtschaftungsform.

Diese Beobachtung war der Anlass, das Projektgebiet mehr als 50 Jahre nach dem Beginn der ersten Versuche zur Hochlagenaufforstung wieder zu untersuchen. Mit einer Nachkartierung des Bodens wurden die historischen Erhebungen mit den neuen Entwicklungen der Bodensystematik in Einklang gebracht (Neuwinger und Czell 1961, Stöhr 2007, Jandl et al. 2008). Außerdem wurden Bodenproben für die chemische Analyse gezogen. Die Böden sind dem geologischen Material zufolge stark sauer und haben eine extrem geringe Kationenaustauschkapazität. Die mikrobielle Biomasse des Bodens ist ebenfalls gering. Alle diese Ergebnisse stimmen mit den Erwartungen an Böden im Hochgebirge gut überein.

Im Rahmen des Projektes wurde auch die Bodentemperatur gemessen (Abb. 6); einerseits um an die historischen Messungen anzuschließen, andererseits weil dieser Parameter ein wichtiger Zeigerwert für die obere Waldgrenze ist (Aulitzky 1963,

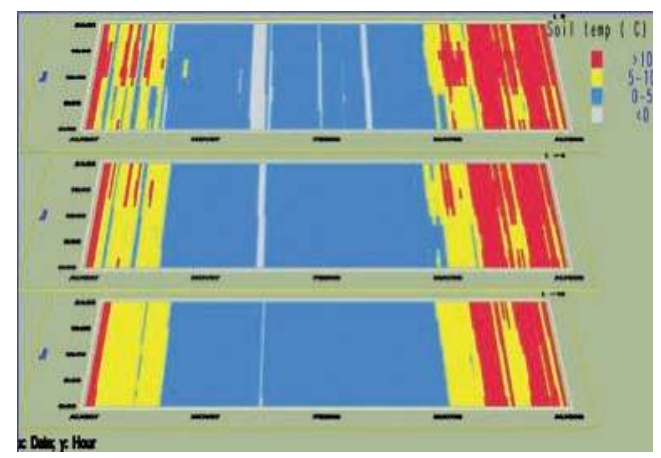

Körner \& Paulsen 2004). Demnach friert der Boden im Winter nur in einer sehr kurzen Zeit. Trotz der extremen Kälte in der freien Atmosphäre übt die Schneedecke ihre isolierende Wirkung aus und ermöglicht den Bodenmikroorganismen sogar die Fortsetzung der Veratmung von Bodenkohlenstoff mit einer geringen Rate (Hubbard et al. 2005, Schindlbacher et al. 2007).

Der biogeochemische Kreislauf des Bodenkohlenstoffes im Waldgrenzen-Ökoton ist noch nicht befriedigend geklärt. In weiteren Forschungsprojekten soll geklärt werden, warum und wie deutlich sich die Kohlenstoffvorräte in den Böden unter Zirbenwald und Zwergstrauchheiden unterscheiden. Dazu werden mehrere Hypothesen verfolgt: (1) die Pflanzengesellschaft beeinflusst die bodenmikrobiellen Prozesse durch die Modifizierung des Kleinklimas, (2) durch die Qualität der gebildeten Streu, (3) durch die Menge des Streufalls, oder der Wechsel der Vegetationsform löst im Boden einen ,priming'Effekt aus (Fontaine et al. 2004, Kuzyakov 2010; Abb. 7).

Abb. 6:

Bodentemperatur am Standort Poschach/Obergurgl oberhalb der aktuellen Waldgrenze in der Zwergstrauchheide. Auf der $\mathrm{x}$-Achse ist das Datum, auf der y-Achse die Uhrzeit eingetragen. Die drei Panele zeigen die Bodentiefen $5 \mathrm{~cm}$ (oben), $15 \mathrm{~cm}$ (Mitte) und $30 \mathrm{~cm}$ (unten). 


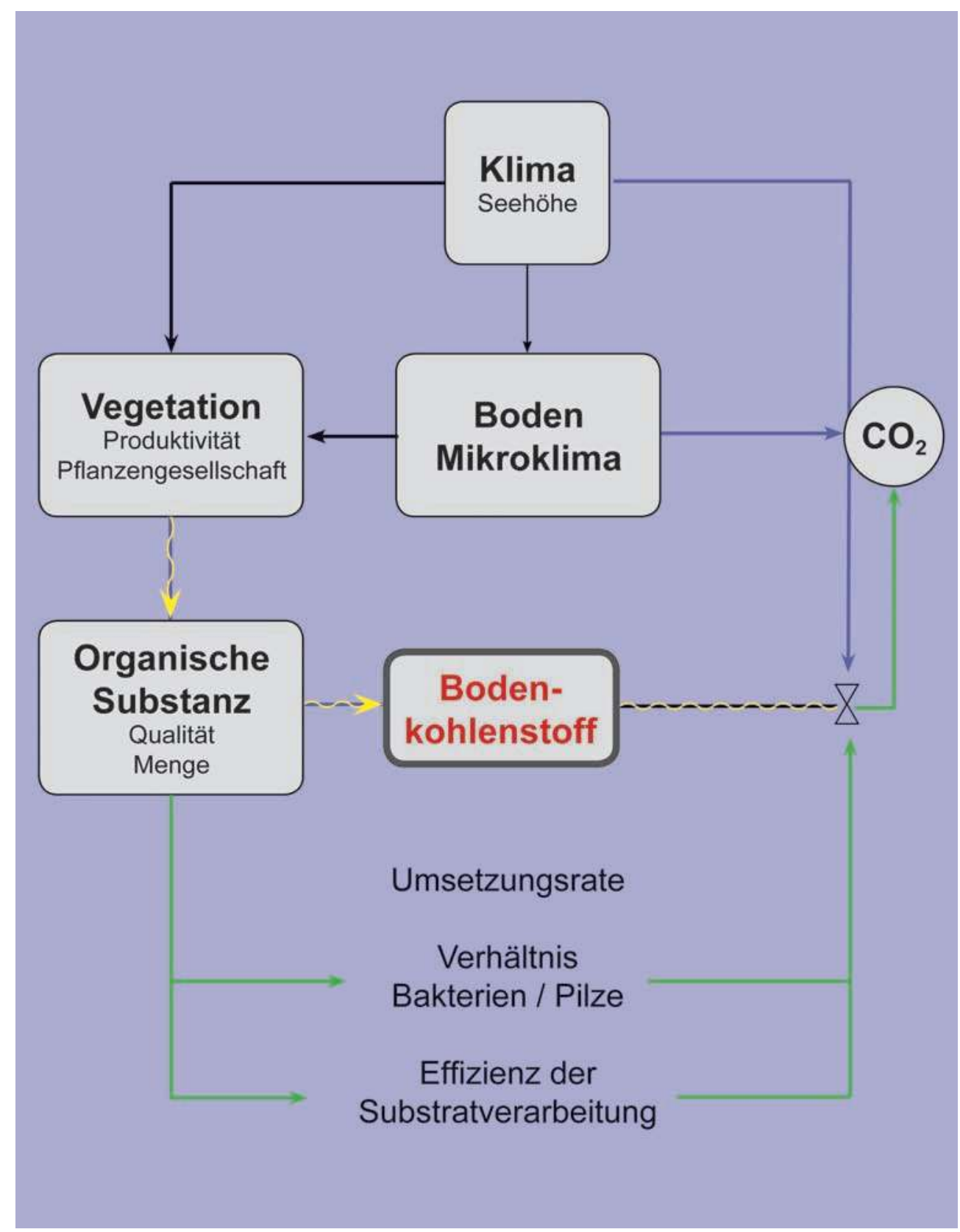

Abb. 7:

Hypothesen über den Zusammenhang zwischen dem Bodenkohlenstoffvorrat, der Vegetation und dem Klima 
Herausforderungen für die Hochlagenaufforstung in der Zukunft

Die wichtigsten Herausforderungen zukünftiger Hochlagenaufforstungen sind der Klimawandel und fortschreitende Landnutzungsänderungen. Der Klimawandel äußert sich in der Erwärmung, der Veränderung der zeitlichen Verteilung und Menge des Niederschlages und in der Veränderung der Häufigkeit von Extremereignissen. Am belastbarsten gelten den Klimaforschern die Temperatur-Szenarien (Abb. 8). Das Intergovernmental
Panel of Climate Change (IPCC 2007) hat mehrere Szenarien der Emission von Treibhausgasen entwickelt, welche zur globalen Erwärmung führen. Szenario A1B zeigt eine Welt mit raschem ökonomischen Wachstum und einer mittelfristig wachsenden Weltbevölkerung, die sich aus einem Mix aus fossilen und erneuerbaren Energiequellen bedient. Szenario B1 unterstellt eine raschere Anpassung der Menschheit an die Herausforderungen des Klimawandels und ist daher eine Variante mit einer langsameren globalen Erwärmung. Die globalen Klimaszenarien haben nur eine sehr grobe räumliche

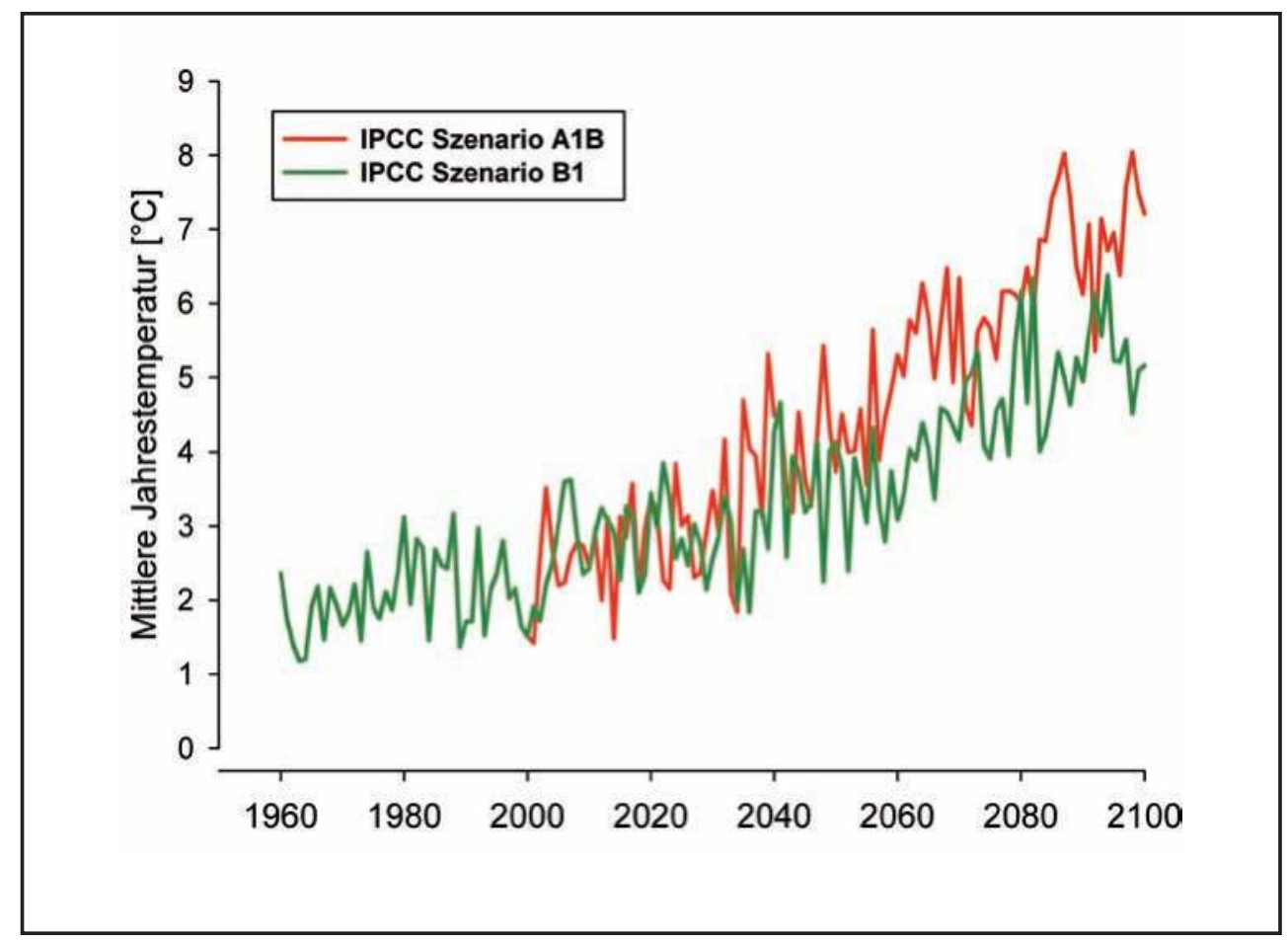

Abb. 8:

Mittlere Jahrestemperatur in Obergurgl nach den Emissionsszenarien A1B und B1 des IPCC 
Auflösung. Daher werden sie regionalisiert. Derzeit kann eine räumliche Auflösung von $10 \times 10 \mathrm{~km}$ erreicht werden. Abb. 8 zeigt bis zum Jahr 2100 eine Temperaturerhöhung zwischen 3 und $5^{\circ} \mathrm{C}$. Ungeachtet der verschiedenen Modellansätze zeigen bereits die Messungen der Vergangenheit, dass der Alpenraum sich stärker erwärmt als im globalen Mittel (Auer et al. 2007): während beispielsweise die globale Temperatur seit Mitte des 19. Jahrhunderts im Mittel um ca. $0,8^{\circ}$ $\mathrm{C}$ gestiegen ist, stiegen die Temperaturen im österreichischen Alpenraum um etwa das Doppelte, um knapp 1,6 $6^{\circ}$ C. Setzt sich dieser Trend in gleichem Maße in der Zukunft fort, so impliziert das große Veränderungen. Zum Beispiel muss bei Aufforstungen der Baumgenetik eine verstärkte Aufmerksamkeit gewidmet werden, denn aufgrund der langen Umtriebszeit von Schutzwäldern werden die Bäume in Zukunft anderen Klimabedingungen ausgesetzt sein, als bei der Pflanzung (Zwerger \& Schüler 2008).

Allein der Temperatureffekt würde einer Verschiebung der Vegetation zwischen 250 und 500 Höhenmetern entsprechen. Versuche mit verschiedenen Baumarten zeigen, dass derzeit die heimischen Baumarten noch das größte Wuchspotential und die höchste Schadresistenz aufweisen (Weißenbacher et al. 2011). Ob dies aber auch unter veränderten Klimabedingungen gilt, kann derzeit noch nicht beantwortet werden. Eventuell wird fremdländischen Baumarten zur Stabilisierung von
Waldgrenzregionen in Zukunft eine höhere Bedeutung zukommen (Zwerger \& Schüler 2008, Abb. 9).

Bei der Stabilisierung der Schutzwälder und der Anpassung an veränderte Umweltbedingungen spielt neben den Aufforstungsmaßnahmen der natürliche Anstieg der Baumgrenze eine wichtige Rolle. Dabei stellt sich die Frage, ob die natürlichen Prozesse, die einen schnellen Anstieg der Baumgrenze ermöglichen, intakt sind (z.B. Namkoong et al. 1996). Wichtig in diesem Zusammenhang sind die Sicherung der Reproduktion der Bäume (z.B. Auftreten von Samenjahren) und das Vorhandensein von Samen ausbreitenden Vektoren (z.B. Tannenhäher für die Zirbe). Aus wissenschaftlicher Sicht stellt sich die Frage, wie groß die Zeitverzögerung zwischen Klimaerwärmung einerseits und den natürlichen Ausbreitungsprozessen an der Waldgrenze andererseits ist.

Herausforderungen für die Bewirtschaftung des Waldes in Hochlagen in der Zukunft

Der Wald ist vielfach unter Druck. Die oben beschriebenen klimatischen Stressoren beeinflussen auch die potentielle biotische Schädigung der Wälder. Unter künftigen Klimabedingungen werden Schädlinge ihr Verbreitungsgebiet ausbauen können. Schon jetzt ist etwa 


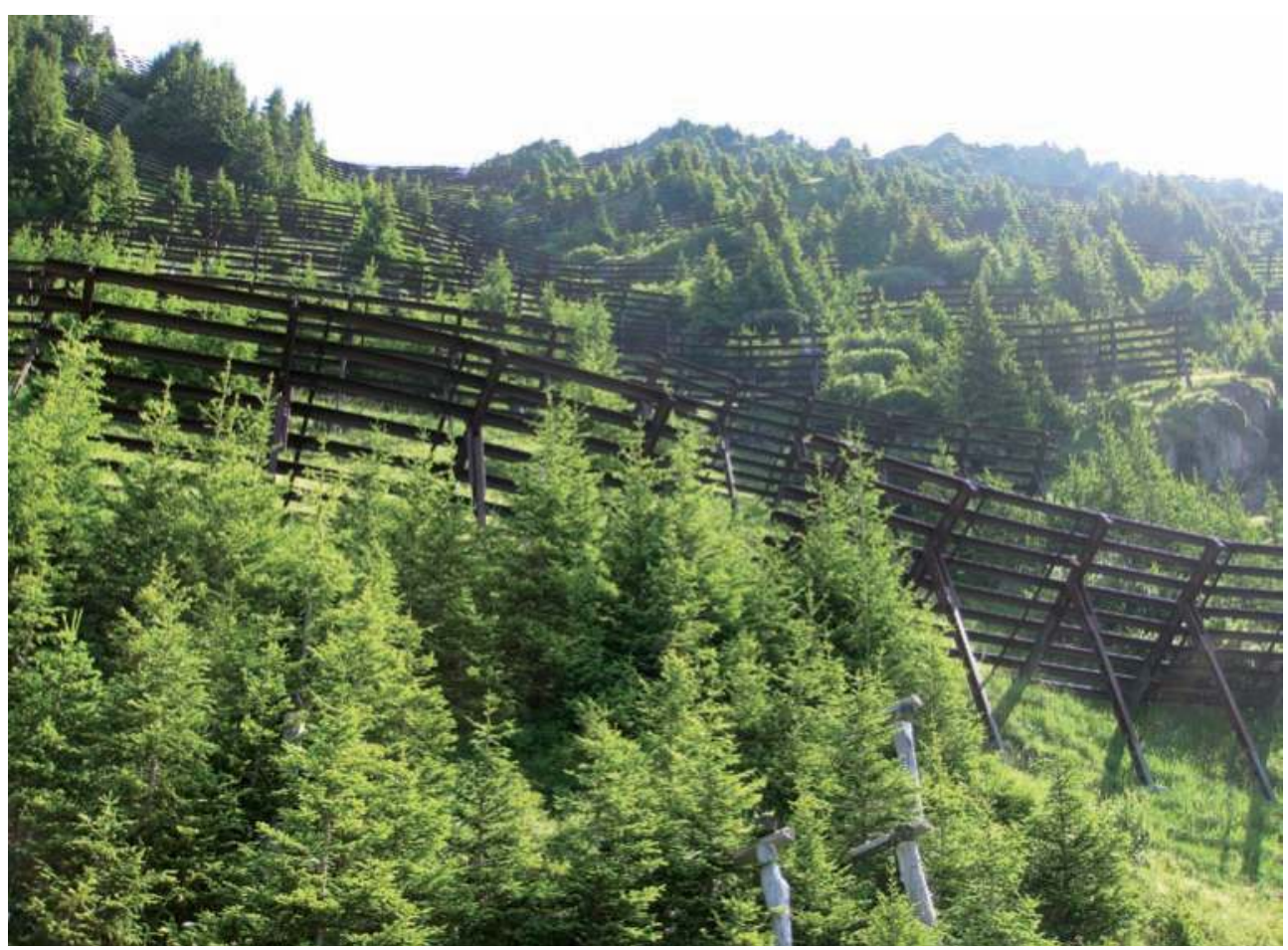

Abb. 9:

Hochlagenaufforstungen als biologische Verbauungen sind ein unverzichtbarer Bestandteil von Lawinenschutzmaßnahmen. Das Foto zeigt eine Fichtenstecklingsfläche in etwa 2200 m Seehöhe bei Kappl (Tirol). (Foto: Lambert Weißenbacher)

der Borkenkäfer als Fichtenschädling in Regionen anzutreffen, die in klassischen Lehrbüchern (z.B. Schwerdtfeger 1981) als ,nicht gefährdet' galten. Zur heimischen Schädlingspopulation ist noch mit einer neu einwandernden Population zu rechnen. Das Bild wird zunehmend komplexer, wenn man auch bedenkt, dass zudem Antagonisten und Protagonisten wirksam werden. Ein Szenario über die künftige forstentomologische Situation ist daher extrem spekulativ. Ein weiterer Schadfaktor sind Sturmschäden, von de- nen behauptet wird, dass sie im Zuge des Klimawandels häufiger werden (Gardiner et al. 2010). Für die Schutzwälder der höheren Lagen sind diese Schadfaktoren besonders gefährlich, denn die phytosanitäre Aufarbeitung von Schadholz ist in diesen Lagen kaum wirtschaftlich zu bewältigen. Diese Störungen der Waldökosysteme beeinträchtigen unmittelbar die Schutzfunktion der Wälder, sie vermindern aber auch die Kohlenstoffspeicherung im Wald und verändern die Hydrologie (Körner 2003b). Die natürlichen 
Belastungsfaktoren werden von der Unsicherheit der künftigen Bewirtschaftungsintensität der Wälder überlagert. Die geringer werdende Kapazität an Arbeitskräften, die Erschließung neuer Flächen für den Ski-Tourismus, das Verhalten der zunehmend, waldfernen' Waldbesitzer und die Waldschädigung infolge der hohen Wilddichte lassen nicht erwarten, dass die traditionelle Form der intensiven Waldbewirtschaftung fortgesetzt wird (Haas 2008, Hogl et al. 2005, Jandl \& Stöhr 2009, Stöhr 2009). Vielmehr gilt es, Formen der ,adaptiven Waldbewirtschaftung' zu finden, die davon ausgehen, dass sich das Klima der Zukunft deutlich vom heutigen Klima unterscheidet und die Schutzfunktion des Waldes dennoch permanent erhalten bleibt. Diese Konzepte werden derzeit in enger internationaler Kooperation zwischen Praktikern und Wissenschaftlern entwickelt (Heinimann 2010, Lindner et al. 2010, Seppälä et al. 2009). Die Erwartung, dass der Wald wesentlich zur Milderung des Klimawandels (,mitigation') beiträgt, wird nicht mehr unterstützt. Vielmehr stellt sich die Frage, wie der Wald bestmöglich an die geänderten Standortbedingungen angepasst werden sollte (Brang et al. 2008).

Angesichts des geringer werdenden wirtschaftlichen Anreizes der Waldbewirtschaftung für die Holzproduktion, kann die Hoffnung einer multifunktionalen Waldwirtschaft, in welcher Ökosystemleistungen als erfreuliches Nebenprodukt erbracht werden, nicht unbedingt auf- recht erhalten werden. Vielmehr ist damit zu rechnen, dass die Erhaltung einer geeigneten Waldstruktur zur Erzielung der Schutzfunktion vermehrte Investitionen benötigen wird (Broggi 2002, Puettmann et al. 2009).

\section{Literatur}

Amt der Tiroler Landesregierung (ADTLR, 2011) Tiroler Waldbericht 2011. http:// www.tirol.gv.at/fileadmin/www.tirol. gv.at/themen/umwelt/wald/waldzustand/downloads/waldbericht2011.pdf, 27. 9. 2011.

Aulitzky H. (1961) Die Bodentemperaturen in der Kampfzone oberhalb der Waldgrenze und im subalpinen LärchenZirben-Wald. In: Ökologische Untersuchungen in der subalpinen Stufe - Teil 1, Mitteilungen der Forstlichen BundesVersuchsanstalt 59: 153-208.

Aulitzky H. (1963) Grundlagen und Anwendung des vorläufigen Wind-SchneeÖkogrammes. In: Ökologische Untersuchungen in der subalpinen Stufe - Teil 2, Mitteilungen der Forstlichen BundesVersuchsanstalt 60: 765-834.

Aulitzky H. (1992) Die Sprache der ,'stummen Zeugen “. Proceedings Interpraevent 1992: 139-174.

Brang P., Bugmann H., Bürgi A., Mühlethaler U., Rigling A. \& Schwitter R. (2008) Klimawandel als waldbauliche Heraus- 
forderung. Schweizerische Zeitschrift für das Forstwesen 159: 362-373.

Broggi M. F. (2002) Alpine Space: A challenge for research. Centralblatt für das gesamte Forstwesen 119: 247-254.

Ellenberg H., Mayer R. \& Schauermann J. (1986) Ökosystemforschung - Ergebnisse des Sollingprojektes 1966-1986. Eugen Ulmer Verlag, Stuttgart

Erschbamer B. (2007) Vegetation at the upper timberline. In: Trees at their upper limit - Treelife limitation at the Alpine Timberline, Chapter 5, Wieser G., Tausz M. (Hg.) Plant Ecophysiology Vol 5: 67-78.

Fliri F. (1973) Beziehungen zwischen Mensch und Wald im Gebirgsland Tirol. Allgemeine Forstzeitung 84: 268-271.

Fontaine S., Bardoux G., Abbadie L., Mariott A. (2004) Carbon input to soil may decrease soil carbon content. Ecology Letters: 314-320.

Forstliche Bundes-Versuchsanstalt Mariabrunn (Hg.) (1961, 1963) Ökologische Untersuchungen in der subalpinen Stufe - Teil 1 und 2, Mitteilungen der FBVA, Band 59 und 61.

Friedel H. (1961) Schneedeckendauer und Vegetationsverteilung im Gelände. In: Ökologische Untersuchungen in der subalpinen Stufe - Teil 1, Mitteilungen der Forstlichen Bundes-Versuchsanstalt 59: 317-369.

Fromme G. (1957) Der Waldrückgang im Oberinntal (Tirol). Untersuchungen über das Ausmaß, die Ursachen und Folgeerscheinungen des Waldrückganges in einem Gebirgslande sowie über die Aussichten der Wiederaufforstung. Mitteilungen der Forstlichen BundesVersuchsanstalt Mariabrunn 54: 1-220.

Gardiner B., Blennow K., Carnus J.-M., Fleischer P., Ingemarson F., Landmann G., Lindner M., Marzano M., Nicoll B., Orazio C., Peyron J.-L., Reviron M.P., Schelhaas M.-J., Schuck A., Spielmann M., Usbeck T. (2010) Destructive Storms in European forests: past and forthcoming impacts. European Forestry Institute (EFI), Joensuu.

Haas W. (Projektleitung 2008) Integrative Forschung im Ötztal. Footprints - future - past - present. Endbericht MaB Biosphärenpark Ötztal. Phase 2. Institute for Social Ecology. Faculty for Interdisciplinary Studies, Klagenfurt University.

Hegg C., Badoux A., Lüscher P., Witzig J. (2004) Zur Schutzwirkung des Waldes gegen Hochwasser. Forum für Wissen 2004: 15-20.

Heinimann H. R. (2010) A concept in adaptive ecosystem management - An engineering perspective. Forest Ecology and Management 259: $848-856$.

Hogl K., Pregernig M., Weiss G. (2005) What is new about new forest owners? A typology of private forest ownership in Austria. Small-scale Forestry 4: 325-342.

Hubbard R. M., Ryan M. G., Elder K., Rhoades C. C. (2005) Seasonal patterns in soil surface $\mathrm{CO}_{2}$ flux under snow cover in 50 and 300 year old subalpine forests. Biogeochemistry 73: 93-107.

IPCC (2007) Climate Change 2007: Synthesis Report. Contribution of Working 
Groups I, II and III to the Fourth Assessment Report of the Intergovernmental Panel on Climate Change [Core Writing Team, Pachauri, R.K and Reisinger, A. (Hg.)]. IPCC, Geneva, Switzerland, $104 \mathrm{~S}$.

Jandl R., Schindlbacher A., Katzensteiner K., Göttlicher S., Katzensteiner H., Zechmeister-Boltenstern S., Pfeffer M., Stöhr D. (2008) Effect of a climateinduced shift of the timberline on the release of greenhouse gases - Report StartClim.2007.D. Federal Forest Research Center (BFW), Vienna.

Jandl R., Stöhr, D. (2009) Forests and rural development in the light of global change - a perspective of mountain forests. Folia Forestalia Polonica Series A, 51: 33-38.

Johann E (1986) Nutzung, Gefährdung und Schutz des Waldes in der Geschichte der Gebirgsregionen der Ostalpen. International Union of Forest Research Organizations, Proceedings des 18. IUFRO Weltkongress: 238-244.

Johann E. (2007) Traditional forest management under the influence of science and industry: The story of the alpine cultural landscapes. Forest Ecology and Management 249: 54-62.

Kilian W., Müller F., Starlinger F. (1994) Die Forstlichen Wuchsgebiete Österreichs. Berichte der Forstliche Bundesversuchsanstalt Wien, Band 82: 1-60.

Körner C. (2003a) Alpine plant life - functional ecology of high mountain ecosystems. Springer Verlag, New York.
Körner C. (2003b) Slow in, rapid out - Carbon flux studies and Kyoto targets. Science 300: 1242-1243.

Körner C., Paulsen J. A. (2004) World-wide study of high altitude treeline temperatures. Journal of Biogeography 31: 713-732.

Kuzyakov Y. (2010) Priming effects: Interactions between living and dead organic matter. Soil Biology and Biochemistry 42: $1363-1371$.

Larcher W. (2001) Ökophysiologie der Pflanzen: Leben, Leistung und Streßbewältigung der Pflanzen in ihrer Umwelt. UTB, Stuttgart.

Lindner M., Maroschek M., Netherer S., Kremer A., Barbati A., Garcia-Gonzalo J., Seidl R., Delzon S., Corona P., Kolström M., Lexer M. J. \& Marchetti M. (2010) Climate change impacts, adaptive capacity, and vulnerability of European forest ecosystems. Forest Ecology and Management 259: 698 - 709 .

Likens G.E. \& Bormann F.H. (1995) Biogeochemistry of a Forested Ecosystem. Springer Verlag, New York.

Kronfuss H. (1984) Die Hochlagenaufforstung Haggen. Der Tiroler Forstdienst 27: 4-6.

Markart G. (2000) Der Wasserhaushalt von Hochlagenaufforstungen - Dargestellt am Beispiel der Aufforstung von Haggen bei St. Sigmund im Sellrain. Berichte der FBVA117: 1-126.

Markart G., Sauermoser F., Kohl B., Stern R. (2002) Land-use changes and the effect of mountain forests in alpine catch- 
ments. Centralblatt für das gesamte Forstwesen 119: 335-343.

Matzner E. (Hg.) (2004) Biogeochemistry of forested catchments in a changing environment - A German case study, Ecological Studies Vol. 172, Springer Verlag, New York.

Mayer H. (1976) Gebirgswaldbau - Schutzwaldpflege. Gustav Fischer Verlag, Stuttgart.

Moser W., Peterson, J. (1981) Limits to Obergurgl's growth. Ambio 10: 68-72.

Müller F., Schultze U. (1996) Einfluss einer Korngrößensortierung auf Frühtestmerkmale und Sämlingsentwicklung von Nachkommenschaften aus FichtenSaatguterntebeständen unterschiedlicher Seehöhe. Centralblatt für das gesamte Forstwesen 113: 155-174.

Naidoo R., Balmford A., Costanza R., Fisher B., Green R. E., Lehner B., Malcolm T. R., Ricketts T. H. (2008) Global mapping of ecosystem services and conservation priorities. PNAS 105: 9495-9500.

Namkoong G., Boyle T., Gregorius H.-R., Joly H., Savolainen O., Ratnam W., Young A. (1996) Testing criteria and indicators for assessing the sustainability of forest management: Genetic criteria and indicators. Working Paper No. 10, Center for International Forestry Research, Bogor, Indonesia.

Neuwinger-Raschendorfer, I. \& Czell, A. (1961) Böden in den Tiroler Zentralalpen. Mitteilungen der Forstlichen Bundes-Versuchsanstalt Mariabrunn 59: 371-410.
Puettmann K.J., Coates K.D., Messier, C. (2009) A critique of silviculture - managing for complexity. Island Press.

Plattner E. (1983) Die Entwicklung des Berglandes - Gefahr für den Fortbestand alpiner Wälder. Wald- und Holzwirtschaft 361: 140-142.

Sauermoser S. (1988) Bisherige Erfahrungen in der Hochlagenaufforstung der Wildbach- und Lawinenverbauung in Tirol in den letzten 35 Jahren: Schutz des Lebensraumes vor Hochwasser, Muren und Lawinen. Proceedings INTERPRAEVENT Graz: 253-266.

Schindlbacher A., Zechmeister-Boltenstern S., Glatzel G., Jandl, R. (2007) Winter soil respiration from an Austrian mountain forest. Agricultural and Forest Meteorology 146: 205-215.

Schreiber M. (1957) Zur Planung der Aufforstungen in der subalpinen Stufe der österreichischen Alpen. Allgemeine Forstzeitung 68: 132-137.

Schröter D., Cramer W., Leemans R., Prentice I. C., Araújo M. B., Arnell N. W., Bondeau A., Bugmann H., Carter T. R., Gracia C. A., de la Vega-Leinert A. C., Erhard M., Ewert F., Glendining M., House J. I., Kankaanpää S., Klein R. J. T., Lavorel S., Lindner M., Metzger M. J., Meyer J., Mitchell T. D., Reginster I., Rounsevell M., Sabaté S., Sitch S., Smith B., Smith J., Smith P., Sykes M. T., Thonicke K., Thuiller W., Tuck G., Zaehle S. \& Zierl B. (2005) Ecosystem Service Supply and Vulnerability to Global Change in Europe. Science 310: 1333-1337. 
Schwerdtfeger F. (1981) Die Waldkrankheiten. Ein Lehrbuch der Forstpathologie und des Forstschutzes. Verlag Paul Parey, Hamburg.

Seppälä R., Buck A., Katila, P. (Hg.) (2009) Adaptation of forests and people to climate change. A Global Assessment Report. IUFRO World Series Vol 22, Helsinki.

Spangenberg J. H., Settele J. (2010) Precisely incorrect? Monetising the value of ecosystem services. Ecological Complexity 7: $327-337$

Stauder, S. (1963) Das Projekt 'Wildbachund Lawinenvorbeugung Vorderes Zillertal' und seine wirtschaftliche Bedeutung. Mitteilungen der Forstlichen Bundes-Versuchsanstalt Mariabrunn 60/ II: 721-741.

Stauder S. (1968) Methoden und Erfahrungen der Integralmeliorationen in Tirol. Allgemeine Forstzeitung 79: 196-198.

Stern R., Zwerger P. (1980) Rumelische Kiefer (Pinus peuce Griseb.) und Zirbe (Pinus cembra L.): Ein Pflanzvergleich in der subalpinen Stufe Tirols. Mitteilungen der Forstlichen Bundesversuchsanstalt Wien 129: 27-40.

Stöhr D. (2007) Soils - heterogeneous at the microscale. In: Trees at their upper limit - Treelife limitation at the Alpine Timberline, Chapter 3, Wieser G., Tausz, M. (Hg.), 37-56, Plant Ecophysiology Vol 5, Springer, Dordrecht.

Stöhr D. (2009) Is there a future for mountain forests? In: Global Change and Sustaina- ble Development in Mountain Regions, Jandl R., Borsdorf A., Van Miegroet H., Lackner R., Psenner R. (Hg.) innsbruck university press Vol 7: 31-38.

Thuiller W., Lavorel, S., Araújo M. B., Sykes M. T., Prentice I. C. (2005) Climate change threats to plant diversity in Europe. PNAS 102: 8245-8250.

Tranquillini W. (1979) Physiological ecology of the alpine timberline - Tree existence at high altitude with special reference to the European Alps. Ecological Studies Vol 31, Springer, New York.

Turner H. (1961) Die Niederschlags- und Schneeverhältnisse. In: Ökologische Untersuchungen in der subalpinen Stufe - Teil 1, Mitteilungen der Forstlichen Bundes-Versuchsanstalt 59: 265-315.

Weißenbacher L., Herz H., Schüler S., Zwerger P. (2007) Fichtenstecklinge - Eine Alternative für Hochlagenaufforstungen. Forstzeitung 118: 36-38.

Weißenbacher L., Schüler S., Herz H., Zwerger P. (2011) Heimische und fremdländische Baumarten an der Waldgrenze. Forstzeitung 122: 14-16.

Wieser G., Tausz M. (2007) Trees at their upper limit - Treelife limitation at the Alpine Timberline. Plant Ecophysiology Vol. 5, Springer, New York.

Zwerger P., Schüler S. (2008) Stabile Schutzwälder - eine Herausforderung für die Forstgenetik. Österreichische Forstzeitung 15: 19-21. 
Robert Jandl

Bundesforschungs- und Ausbildungszentrum

für Wald, Naturgefahren und Landschaft (BFW)

Seckendorff-Gudent-Weg 8, 1131 Wien

robert.jandl@bfw.gv.at

Andreas Schindlbacher

Bundesforschungs- und Ausbildungszentrum

für Wald, Naturgefahren und Landschaft (BFW)

Seckendorff-Gudent-Weg 8, 1131 Wien

andreas.schindlbacher@bfw.gv.at
Silvio Schüler

Bundesforschungs- und Ausbildungszentrum

für Wald, Naturgefahren und Landschaft (BFW)

Seckendorff-Gudent-Weg 8, 1131 Wien

silvio.schueler@bfw.gv.at

Dieter Stöhr

Landesforstdirektion

Amt der Tiroler Landesregierung

Bürgerstraße 36, 6020 Innsbruck

Dieter.Stoehr@tirol.gv.at 


\section{Kapitel 6 I Jahresringuntersuchungen an rezentem und subfossilem Holzmaterial aus dem Raum Obergurgl -}

\section{Klimaanalysen und holozäne Waldentwicklung}

Kurt Nicolussi, Andrea Thurner

\section{Zusammenfassung}

Im Gebiet von Obergurgl wurden Bohrkerne lebender Zirben (Pinus cembra) aus dem Obergurgler Zirbenwald (ca. 2000 - 2100 m Seehöhe) sowie subfossile Holzproben aus dem Zirbenwaldmoor (2050 m Seehöhe) und den kleinen Moorarealen unterhalb der Gurgler Alm (2150 m bis $2240 \mathrm{~m}$ Seehöhe) dendrochronologisch untersucht.

Die Analyse der Jahrringbreiten der rezenten Bäume des Gurgler Zirbenwaldes zeigt Übereinstimmungen des Radialzuwachses mit der Sommertemperaturentwicklung (Juni-JuliAugust-Mittel). Die Jahrringdaten von 36 subfossilen Hölzer aus dem Zirbenwaldmoor und den umgebenden Moorflächen decken den Zeitraum zwischen etwa 100 und 1300 n. Chr. ab. Diese Konzentration ist einerseits auf die lokale Moorentwicklung, andererseits auf verschiedene Phasen anthropogener Eingriffe in den umgebenden Waldbestand zurückzuführen. Im Gegensatz dazu spiegelt die zeitliche Verteilung der 23 subfossilen Hölzer aus den unterhalb der Gurgler Alm gelegenen Moor- flächen vor allem die holozäne Waldgrenzentwicklung zumindest in Ansätzen wider.

\section{Abstract}

In the region of Obergurgl cores from living stone pine trees (Pinus cembra) from the so called Obergurgler Zirbenwald (approx. 2000 - $2100 \mathrm{~m}$ a.s.l.) as well as sub-fossil logs found in the peat of the Zirbenwaldmoor $(2050 \mathrm{~m}$ a.s.l) and the small peat bogs below the Gurgler Alm (2150 m to $2240 \mathrm{~m}$ a.s.l.) were investigated by dendrochronological methods.

The analysis of cores from living trees of the Obergurgler Zirbenwald verifies the expected accordance of tree-ring growth and summer temperature evolution (June-July-August average). Tree-ring series established for 36 sub-fossil samples from the Zirbenwaldmoor cover the time period between ca. 100 and ca. $1300 \mathrm{AD}$. This distribution is on the one hand due to the development of the local peat bog and on the other hand caused by anthro- 
pogenic interventions at the encircling forest area. In contrast to the sub-fossil samples of the Zirbenwaldmoor the temporal distribution of 23 wood samples from the peat bogs below the Gurgler Alm is to some extent related to the Holocene tree-line evolution.

\section{Einleitung}

Der Obergurgler Zirbenwald ist einer der Ausgangspunkte der dendrochronologischen Forschung in Österreich: Veronika Giertz (später: Veronika SiebenlistKerner) entnahm hier im Sommer 1970 insgesamt 24 rezenten Zirben (Pinus cembra) Bohrkerne, die dem Aufbau einer ersten Zirben-Jahrringbreitenchronologie dienten (Siebenlist-Kerner 1984). Diese Jahrringchronologie, ergänzt mit an historischen Hölzern des Ötztales gewonnenen Daten, reichte schließlich bis ins Jahr 1466 n. Chr. zurück (Giertz \& Grießer 1975, Siebenlist-Kerner 1984). Diese dendrochronologischen Analysen erfolgten auf Initiative der Alpinen Forschungsstelle, damals unter der Leitung von Walter Moser, und wurden in den Folgejahren ergänzt durch die Erarbeitung von Jahrringchronologien für die beiden anderen wesentlichen alpinen Hochlagenbaumarten, Lärche (Larix decidua) und Fichte (Picea abies). Diese beiden Chronologien, wiederum beruhend auf Daten von lebenden Bäumen und Hölzern historischer Gebäude von verschiedenen Standorten des mittleren und inneren Ötztales, decken die Zeitbereiche 1333-1974 n. Chr. (Lärche) und 1276-1974 n. Chr. (Fichte) ab (Siebenlist-Kerner 1984). Die Daten der Ötztaler Jahrringchronologien wurden in weiterer Folge für Rekonstruktionen der Sommertemperatur (Eckstein \& Aniol 1981), das Holzmaterial teilweise auch für Holzdichtemessungen (Lamprecht 1981, Esper et al. 2007) verwendet.

Veronika Giertz versuchte bereits in den 1970er Jahren die Zirben-Jahrringchronologie weiter in die Vergangenheit zu verlängern. Dazu analysierte sie fünf Stammscheiben, die subfossilen, im Torf des Zirbenwaldmoores eingebetteten Zirbenstämmen entnommen wurden. Die Bearbeitung dieser Hölzer lag nahe, da dieses Moor mitten im Obergurgler Zirbenwald lokalisiert ist. Allerdings gelang ihr der Anschluss beziehungsweise die Verbindung mit der ins 15. Jh. zurückreichenden Jahrringchronologie nicht (Siebenlist-Kerner 1984).

Heute ist klar, warum die Datierung der fünf subfossilen Zirbenproben nicht glückte: sie stammen aus dem ersten Jahrtausend $\mathrm{AD}$ und datieren damit deutlich älter als der Beginn der Giertz-ZirbenJahrringchronologie. Dies wurde belegbar im Rahmen eines Projektes zum Aufbau einer holozänen Hochlagen-Jahrringchronologie (Nicolussi et al. 2004, 2009). Für diese Chronologie, die derzeit die letzten rund 9100 Jahre durchgehend abdeckt, 
war das Obergurgler Zirbenwaldmoor auch einer der Ansatzpunkte für die Hölzersuche und -gewinnung.

Der vorliegende Text fasst Ergebnisse von Untersuchungen zusammen, die die früheren Arbeiten im Raum von Obergurgl fortführen: einerseits werden Auswertungen von Proben, die in den letzten Jahren an Bäumen des Obergurgler Zirbenwaldes gezogen wurden, und darauf basierende Analysen zur Jahrring-Klima-Beziehung vorgestellt, andererseits wird ein Überblick zu den Ergebnissen der Auswertung subfossiler Hölzer aus Mooren im Obergurgler Raum gegeben.

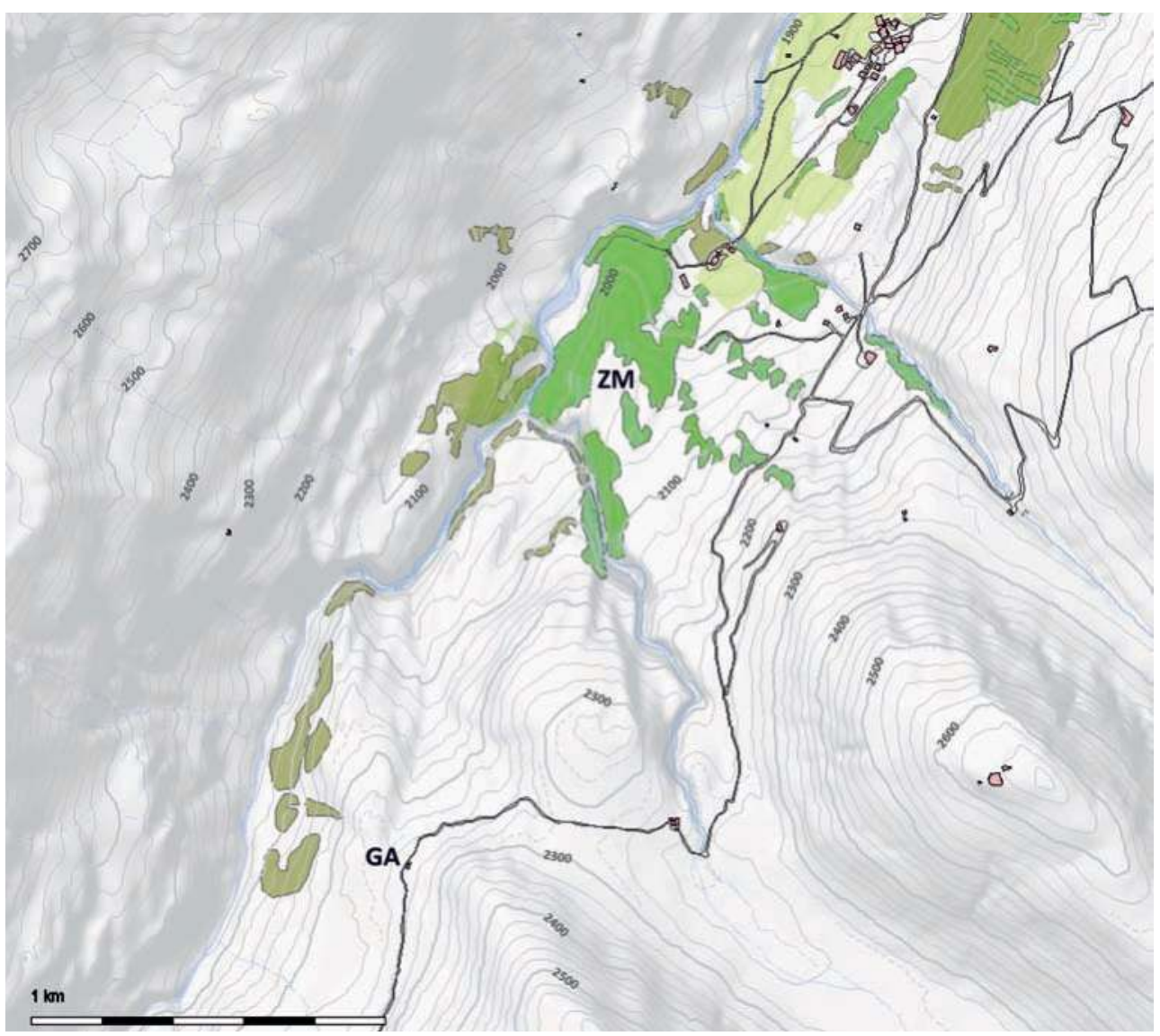

Abb. 1 .

Das Gurgler Tal südlich von Obergurgl mit den ausgewiesenen Waldflächen und den bearbeiteten Moorarealen. ZM: Zirbenwaldmoor, umgeben vom Obergurgler Zirbenwald; GA: Gurgler Alm.

Basiskarte: (C) Land Tirol - tiris 


\section{Material und Methoden}

Insgesamt 92 lebenden Bäumen im Obergurgler Zirbenwald (ca. 2000 - 2100 m Seehöhe, Abb. 1) wurden in den Sommern 1992 bis 2009 Bohrkerne mittels Zuwachsbohrern entnommen. Die Probennahmen konzentrierten sich auf die vergleichsweise alten Bäume des Waldes, nur verhältnismäßig wenig junge Bäume wurden einbezogen. Je Baum wurden in Brusthöhe zwei Bohrkerne hangparallel und von gegenüberliegenden Seiten gewonnen. Da manche Zirben des Obergurgler Zirbenwaldes einen morschen
Kernbereich aufweisen beziehungsweise wenn bei der Beprobung die Stammmitte nicht getroffen wurde, fehlten an vielen Bohrkernen die frühen Jahrringe. Um für solche Proben trotzdem das Lebensalter der einzelnen Bäume zu erfassen und das Kambialalter des ersten gemessenen Jahrrings zu bestimmen, wurde die Anzahl der fehlenden Jahrringe bis zum Mark einerseits anhand von Baumumfangmessungen während der Beprobungen und andererseits mittels der Kurvengravur der Jahrringe am innersten Abschnitt der Bohrkerne abgeschätzt.

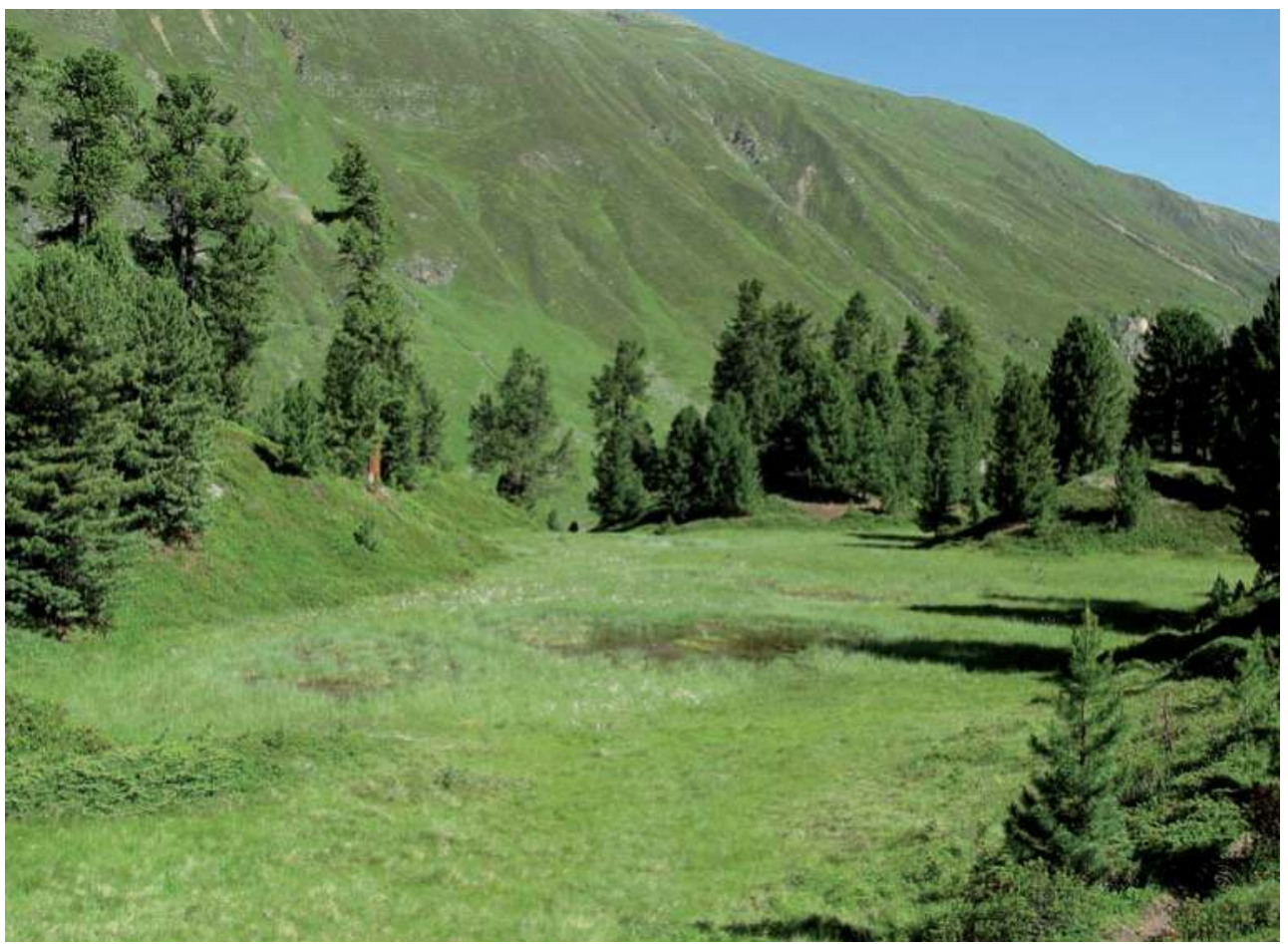

Abb. 2:

Das Obergurgler Zirbenwaldmoor (Foto: Kurt Nicolussi, 2008) 
Die Suche nach subfossilen Hölzern im Bereich von Obergurgl wurde in zwei Moorarealen durchgeführt. Ein Bereich war das Zirbenwaldmoor (11 00 '54" / $46^{\circ} 51^{\prime} 31^{\prime \prime}$, Abb. 2) auf 2050 m Seehöhe mit einigen kleineren, wenig höher liegenden Moorflächen. Das zweite Moorareal umfasst vor allem das unmittelbar unterhalb der Gurgler Alm (11 $1^{\circ} 00^{\prime} 25^{\prime \prime} /$ 46 50`47“, Abb. 3) auf 2240 m Seehöhe gelegene Moor sowie kleinere, nördlich davon und etwas tiefer, am Abhang Richtung Gurgler Ache, liegende Moorflächen (bis ca. 2150 m Seehöhe). In den Mooren erfolgte die Suche nach im Torf eingebet- teten und an der Oberfläche nicht sichtbaren Stämmen mittels rund $90 \mathrm{~cm}$ langer Metallsonden. Dadurch beschränkte sich die Probennahme auf die obersten Torfbereiche. Von den gefundenen Stämmen wurden jeweils Stammscheiben gesägt. An Proben mit fehlenden inneren Holzabschnitten wurde die Distanz sowie die Zahl der fehlenden Ringe bis zum Mark anhand der Jahrringkrümmung geschätzt. Die Ausmessung der Jahrringbreiten der Bohrkernproben und der Stammscheiben erfolgte mit einer Auflösung von 0.001 mm. Die einzelnen Messradien je Baum beziehungsweise je Stammscheibe wur-

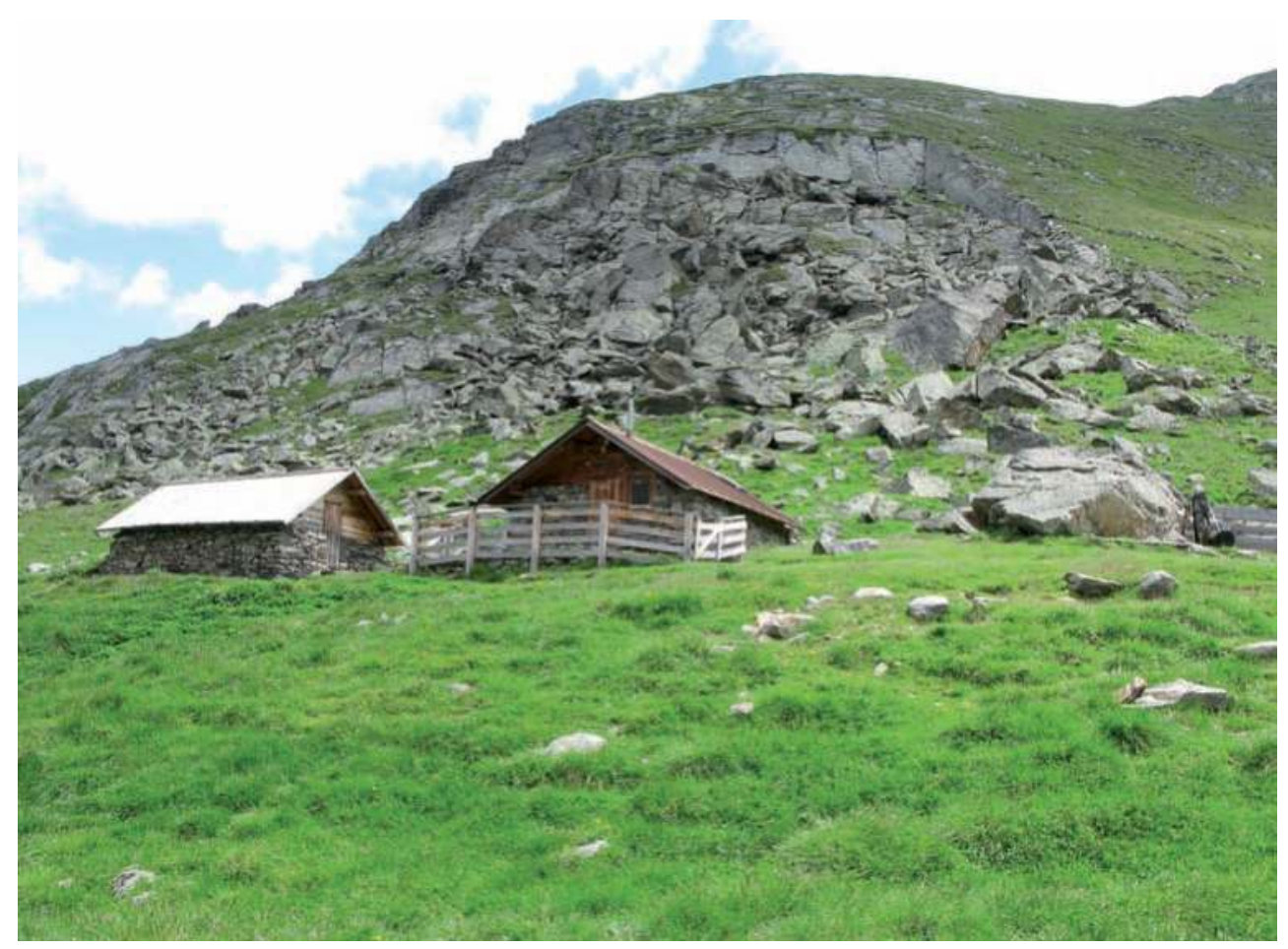

Abb. 3:

Die Gurgler Alm (Foto: Kurt Nicolussi, 2008) 
den nach Überprüfung der Radien zu Baum/Stammmittelkurven zusammengefügt. Die weitere Bearbeitung erfolgte auf Basis dieser Mittelkurven.

Die dendrochronologische, d.h. jahrgenaue Datierung der Jahrringserien der subfossilen Holzproben erfolgte im Zuge des Aufbaus der Ostalpinen Nadelholzchronologie (Nicolussi et al. 2009). Teilweise wurden einzelne Hölzer auch ${ }^{14} \mathrm{C}$ datiert (Daten dazu in Nicolussi et al. 2009). Dies erfolgte speziell am Anfang der Arbeiten für das Hochlagen-Chronologie-Projekt.

Für den Vergleich der rezenten Jahrringdaten mit instrumentellen Klimawerten wurden die Jahrringserien standardisiert, um baumalterbedingte Wachstumstrends in den Reihen zu eliminieren. Grundsätzlich können solche Standardisierungen für jede Jahrringreihe individuell gerechnet werden. Diese Vorgangsweise hat jedoch prinzipiell die Eliminierung langfristiger Wachstumstrends zur Folge (Cook et al. 1995). Für den vorliegenden Vergleich wurde der RCS-Ansatz (RCS: Regional Curve Standardization) gewählt (z.B. Briffa et al. 1992, 1996). Dabei erfolgt die Standardisierung der einzelnen Jahrringserien mittels eines empirischen Wachstumsmodells, das abhängig vom Kambialalter für jeden Jahrring einen zu erwartenden Breitenwert vorgibt. Der Vorteil des RCS-Ansatzes im Gegensatz zur individuellen Standardisierung von Jahrringserien liegt im potentiellen Erhalt jener Komponente der Jahrringbreitenvariabilität, die von langfristigen Klimaentwicklungen geprägt ist.

Die Erstellung des für diese Analyse verwendeten Wachstumsmodells erfolgte auf Basis der rezenten Serien aus dem Obergurgler Zirbenwald unter Berücksichtigung des Kambialalters des jeweils ersten gemessenen Jahrrings der Reihen. Die einzelnen Jahrringserien wurden anschließend durch Division mit der Obergurgler Zuwachskurve unter Verwendung des signal-free Ansatzes (Melvin \& Briffa 2008) standardisiert. Die durch Mittelung aus den so standardisierten Reihen erstellte RCS-Chronologie wurde für Vergleiche an instrumentellen Klimadaten skaliert. Dazu wurde das Juni-Juli-August Temperaturmittel gewählt, da sich hierfür nach response function Analysen mit der vorliegenden Chronologie (Ergebnisse nicht gezeigt) und auch nach einer Reihe von anderen Studien (z.B. Büntgen et al. 2005) im Vergleich die besten Übereinstimmungen ergeben.

\section{Ergebnisse und Diskussion}

\section{Rezentproben aus dem Obergurgler Zirbenwald}

Aus 92 Baummittelkurven von lebenden Zirben wurde eine insgesamt 469 Jahre 
(1540-2008) abdeckende Jahrringbreitenchronologie erstellt. Abb. 4a stellt die Belegung dieser Chronologie für den Zeitraum ab 1780 dar. Da die Bohrkernentnahmen in unterschiedlichen Jahren erfolgte, nimmt die Zahl der Proben pro Jahr für die letzten rund fünf Jahre der Chronologie ab. Insgesamt liegt aber die Belegung für die letzten Jahrhunderte bei rund 80 Bäumen. Das verdeutlicht auch die Dominanz alter Bäume im Datenbestand.

Die Abb. $4 b$ und 4c zeigen den Vergleich der an den Temperaturdaten des Zeitabschnittes 1850-2008 skalierten RCSChronologie mit dem Juni-Juli-August Temperaturmittel über den Zeitraum der letzten knapp 230 Jahre (1780-2008). Von besonderem Interesse sind dabei die Ergebnisse für die Jahre ab 2000, da andere alpine Temperaturrekonstruktionen (z.B. Büntgen et al. 2005, 2011) dieses Jahrzehnt nur ansatzweise abdecken. Hingegen sind die Jahrhunderte der frühen Neuzeit bei den genannten Rekonstruktionen besser als bei der Obergurgler Lokalchronologie belegt. Herangezogen für den Vergleich wurden die Temperaturwerte des HISTALP-Datensatzes (Auer et al. 2007) für den nahe gelegenen Gitterpunkt $11^{\circ}$ Ost / $47^{\circ}$ Nord (Böhm et al. 2010). Da für die Berechnung der Gitterpunktdaten relativ großräumig Stationsreihen verwendet wurden (Auer et al. 2007), zeigt ein Vergleich der Gitterpunktserie mit den Reihen des gesamten HISTALP-Raumes kaum Unterschiede.
Abb. 4b stellt den Vergleich zwischen Jahrring- und instrumentellen Daten auf Einzeljahrbasis dar, Abb. 4c hingegen gibt die mit einem 10-jährigen Tiefpass-Filter geglätteten Werte sowie die Mittel über jeweils 25 Jahre wieder. Die Korrelation zwischen den beiden Klimareihen für den Zeitraum von 1850 bis 2008 beträgt 0.62 (Einzeljahre) beziehungsweise 0.82 (10-jährig geglättete Serien). Zwischen etwa 1840 und 1980 zeigen die beiden Serien eine vergleichsweise gute Übereinstimmung, die Abweichungen fallen relativ gering aus. Allerdings erreichen die Übereinstimmungen nicht das Niveau, das bei der Verwendung von regionalen Chronologien festgestellt werden kann (Büntgen et al. 2005). Hier werden die Limitierungen durch die Verwendung einer lokalen Chronologie ersichtlich. Vor der Mitte des 19. Jahrhunderts treten signifikante Unterschiede auf: einerseits liegt die Varianz der instrumentellen Daten klar über jener der Jahrring-basierten Serie, andererseits zeigt letztere deutlich niedrigere Temperaturen auf. In beiden Fällen dürften hier zumindest teilweise Effekte der „Frühen Instrumentellen Periode“ vorliegen, während der Temperaturmessungen noch nicht standardisiert erfolgten (Frank et al. 2007, Böhm et al. 2010). Zwar wurde der hier verwendete HISTALP-Datensatz gegenüber einer früheren Version schon diesbezüglich korrigiert, das Ausmaß der Änderungen dürfte allerdings nach Ausweis der Jahrringdaten zu gering ausgefallen sein. 


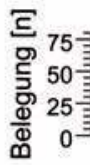

(a)
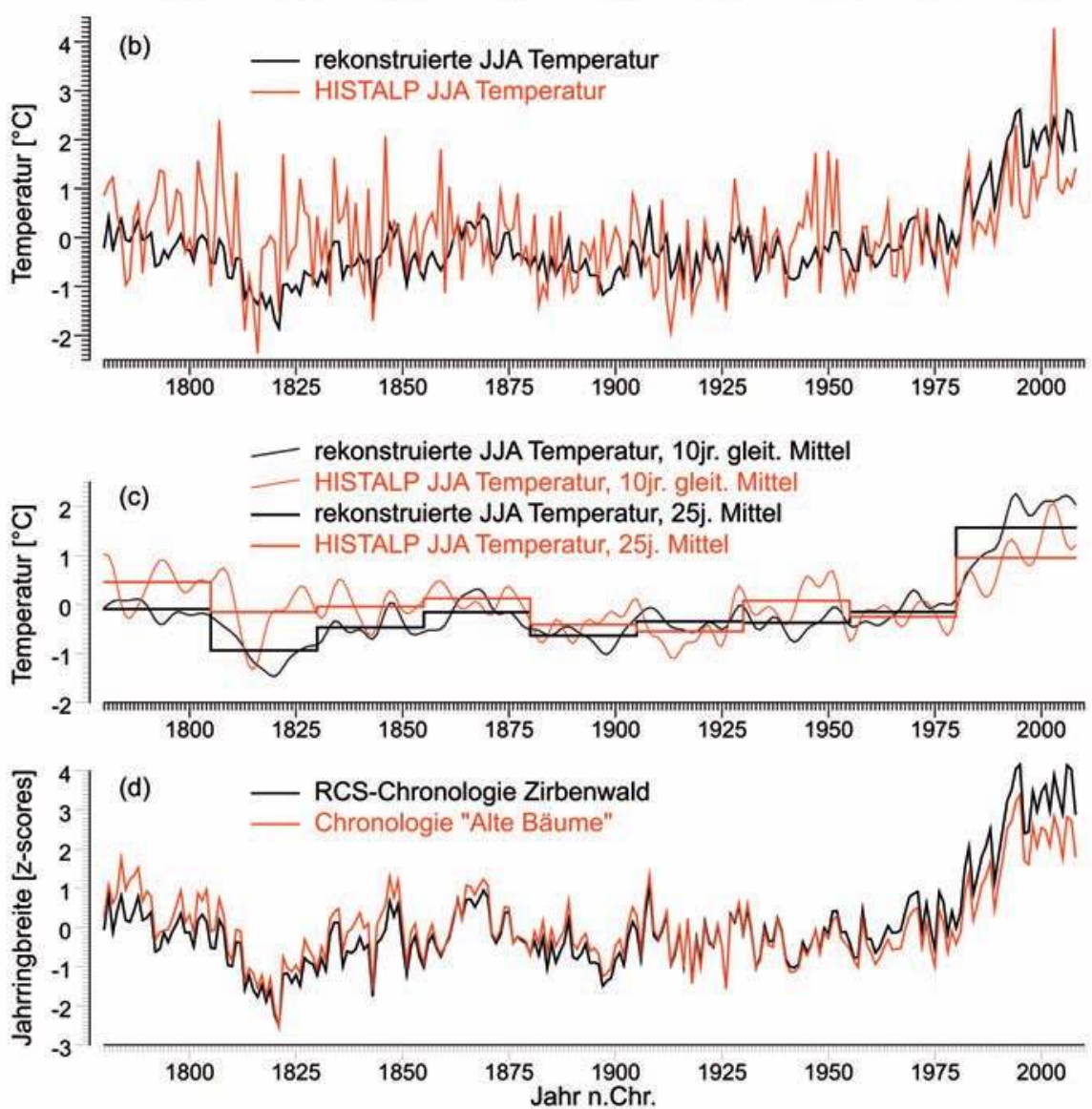

Abb. 4:

Ergebnisse der Untersuchungen an rezenten Zirbenproben des Obergurgler Zirbenwaldes (dargestellter Zeitraum jeweils 1780-2008): a) Anzahl der Serien je Jahr der RCS-Chronologie Zirbenwald; Vergleich der auf Jahrringbasis rekonstruierten sowie der instrumentellen Temperaturreihen (HISTALP Datensatz, Juni-Juli-August-Mittel): b) Einzeljahrbasis und c) mit 10 jährigem Tiefpass-Filter geglättete Serien sowie über jeweils 25 Jahre gemittelt; d) Vergleich der RCS-Chronologie mit der Chronologie "Alte Bäume" (nur Jahrringbreitenwerte mit einem Kambialalter>200) 
Die instrumentellen Sommertemperaturen belegen nach 1980 eine markante Erwärmung an, die von den Jahrringdaten nachgezeichnet und sogar etwas übertroffen wird. Damit zeigt die Obergurgler Zirben-RCS-Chronologie keine Anzeichen eines „divergence-Effektes“ (z.B. D’Arrigo et al. 2008), wie er für andere alpine Jahrringserien postuliert wurde (Oberhuber et al. 2008). Der Grund für diese unterschiedlichen Ergebnisse, erarbeitet jeweils mit Zirben-Chronologien, dürfte in der divergierenden methodischen Herangehensweise zu suchen sein. Um zu überprüfen, ob der starke Anstieg der Zirbenwald-RCS-Chronologie nach 1980 möglicherweise einem Bearbeitungseffekt zuzuschreiben ist, wurde eine zusätzliche Jahrringchronologie ausschließlich auf der Basis der „alten Bäume“ des Datensatzes erstellt. Da die empirischen Wachstumskurven von Zirben $a b$ einem Kambialalter von rund 200 Jahren nur noch einen schwachen Alterstrend aufweisen, wurden an allen Serien die jüngeren Abschnitte (Kambialalter < 200) eliminiert und aus den übrig bleibenden Abschnitten eine Mittelkurve erstellt. Abb. 4d zeigt den Vergleich der RCS-Chronologie mit der Chronologie „alte Bäume“. Beide Serien wurden für den Vergleich über den gleichen Zeitabschnitt standardisiert. Danach ist der starke Wachstumsanstieg der RCS-Chronologie ab 1980 kein methodisches Artefakt, sondern klimainduziert. Die Chronologie „alte Bäume“ zeigt zwar ein etwas geringeres Niveau um 2000, allerdings dürfte sich hier noch ein schwacher Alterstrend bedingter Effekt widerspiegeln, was ebenso für die etwas höheren Werte dieser Chronologie in der ersten Hälfte des Vergleichszeitraums gilt.

\section{Subfossile Hölzer aus dem Zirbenwald- moor}

Für 36 Holzproben von Zirben, gewonnen an subfossilen Stämmen des Zirbenwaldmoores und nahe gelegener Moorareale, liegen dendrochronologische $\mathrm{Da}$ tierungen, d.h. Altersangaben auf Kalenderbasis vor (Abb. 4). Mit den an diesen Proben erarbeiteten Jahrringdaten wird der Zeitraum zwischen 130 und 1280 n. Chr. durchgehend abgedeckt. Die längste einzelne Probenreihe erstreckt sich über 539 Jahre und zählt damit mehr Jahrringe als die längste (469 Jahrringe) an den lebenden Bäumen des Zirbenwaldes gewonnene Serie. Siebenlist-Kerner (1984) erfasste maximal 420 Jahre an einer der lebenden Zirbe.

Bemerkenswert ist, dass trotz der Beprobung aller an der Oberfläche sichtbaren Stämme keine Hölzer aus den letzten sieben Jahrhunderten erfasst wurden. Ebenso auffallend ist der grob übereinstimmende Wachstumsbeginn für rund die Hälfte der subfossilen Proben. Beide Erscheinungen stehen wohl mit der Waldentwicklung in der Umgebung des Zirbenwaldmoores in Zusammenhang und können auf 
Basis einer für dieses Moor vorliegenden Pollenanalyse (Rybnicek \& Rybnickova 1977) erklärt werden. Danach war die Umgebung des Zirbenwaldmoores nach einem ${ }^{14} \mathrm{C}$-Datum (2120 \pm 80 BP, 2 o: 380 cal BC - $30 \mathrm{cal}$ AD) aus einer Tiefe von $100-90 \mathrm{~cm}$ im ausgehenden ersten Jahrtausend v. Chr., vermutlich als Resultat anthropogener Eingriffe, weitgehend entwaldet. Anschließend kam es zu einer Neubildung und Verdichtung der Baumbestände. Gleichzeitig endete auch eine etwa 3500 Jahre andauernde Stagnationsphase des Moorwachstums, während der das Areal stark von Birken bestanden war und die Oberfläche sich nur um 20 bis $30 \mathrm{~cm}$ erhöhte (Rybnicek \& Rybnickova 1977). In der Häufung der Moorhölzer mit einem Reihenbeginn in der frühen Römerzeit (Abb. 5) spiegelt sich danach die Wiederbewaldung wider. Auch das Fehlen älterer Hölzer erklärt sich durch die Moorentwicklung, da die Suche nach Moorhölzern auf den obersten Meter beschränkt war.

Nach der Wiederbewaldung im frühen ersten Jahrtausend n. Chr. und dem Absterben der damals aufgekommenen Bäume zeichnet sich eine grob kontinuierliche Entwicklung von Verjüngung und Absterben ab. Ein klarer Zusammenhang der Waldentwicklung mit der Klimaentwicklung kann nicht hergestellt werden. Einzig das Aufkommen einer Gruppe von Bäumen ab der Mitte des 9. Jahrhunderts könnte als Ausdruck günstiger Klimaver- hältnisse am Beginn der mittelalterlichen Wärmeperiode gesehen werden.

Rybnicek und Rybnickova (1977) gehen von einem kontinuierlichen, ja beschleunigten Moorwachstum über die letzten rund 2000 Jahre aus. Dem steht die Beobachtung gegenüber, dass Stämme von Bäumen, die im Frühmittelalter abgestorben waren und spätestens nach wenigen Jahrhunderten ins Moor gestürzt sein dürften, heute teilweise noch an der Oberfläche sichtbar sind. Gleichzeitig fehlt allerdings Totholz aus den letzten rund 700 Jahren. Anzunehmen ist, dass das Moorwachstum in den letzten Jahrhunderten entgegen den Annahmen von Rybnicek und Rybnickova (1977) wiederum zurückgegangen ist und daher vergleichsweise altes, aus dem ersten Jahrtausend nach der Zeitenwende stammendes Holz auch oberflächennahe vorhanden ist. Gleichzeitig zeigt das Pollendiagramm (Rybnicek \& Rybnickova 1977) in den obersten Dezimetern des Moores wiederum einen deutlichen Waldrückgang. Damit dürften auch nur wenige oder keine Bäume ins Moor gestürzt sein. Dieser mittelalterliche Waldrückgang ist wohl anthropogenen Eingriffen zuzuschreiben, ist doch für spätestens ab dem 13. Jh. eine kontinuierliche Besiedlung und Nutzung dieses Talraumes anzunehmen: eine historische Quelle dokumentiert für das Jahr 1250 durch eine erste Nennung die Existenz eines Hofes in Gurgl. 


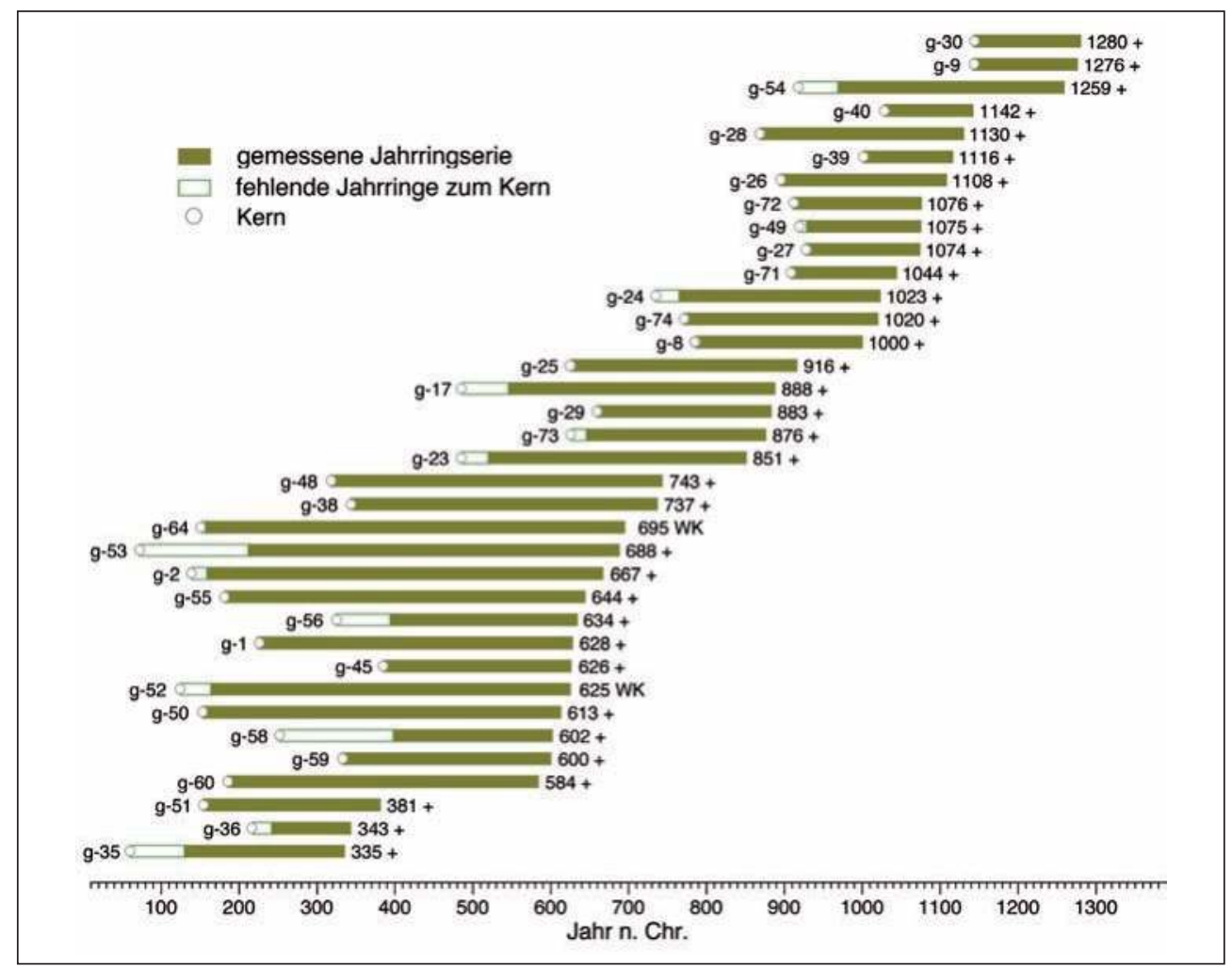

Abb. 5:

Zeitliche Erstreckung der Jahrringserien der datierten subfossilen Hölzer des Obergurgler Zirbenwaldes (2050 m SH). Genannt werden Probenbezeichnung, Endjahr der Jahrringserie und Klassifizierung des letzten gemessenen Jahrringes (+: keine Waldkante; WK: Waldkante vorhanden).

Die Jahrringserien der subfossilen Hölzer aus dem Zirbenwaldmoor bilden einen wichtigen Bestandteil der Ostalpinen Hochlagenchronologie (Nicolussi et al. 2009). Als solche fanden sie bereits Eingang in mehrtausendjährige Temperaturrekonstruktionen (publiziert in Büntgen et al. 2011).
Moorhölzer aus dem Bereich der Gurgler Alm

Das Moor bei der Gurgler Alm (2240 m Seehöhe) und auch die am Abhang nördlich davon und etwas tiefer befindlichen kleinen Moorflächen liegen deutlich höher als das Zirbenwaldmoor und überwie- 
gend im holozänen Schwankungsbereich der Waldgrenze. Schiechtl und Stern (1975) lokalisierten die Obergrenze der potentiellen Zirbenwaldverbreitung in den 1970er Jahren im Bereich der Gurgler Alm bei rund $2200 \mathrm{~m}$ Seehöhe und damit etwa 50 Höhenmeter unterhalb des Almgebäudes. Unter Berücksichtigung des in den letzten Jahrzehnten eingetretenen Temperaturanstieges und dem damit verbundenen verstärkten Aufkommen von Zirbenjungwuchs im Hochlagenbereich (Nicolussi et al. 2005) ist diese Obergrenze heute sicherlich deutlich höher anzusetzen. Damit kann angenommen werden, dass ohne anthropogene Beeinflussung durch Beweidung und bei Andauer der Klimaverhältnisse der letzten zwei Jahrzehnte das kleine Moorareal bei der Gurgler Alm von einem zumindest offenen Zirbenbestand umgeben wäre.

Die Hölzer, die im Moor bei der Gurgler Alm entdeckt werden konnten (Abb. 6), zeigen eindrucksvoll, dass in manchen Abschnitten des Holozäns die Baumverbreitung deutlich über 2250 m Seehöhe hinaus ging. Dies steht in guter Übereinstimmung mit den Ergebnissen einer Pollenanalyse an einem Torfprofil aus diesem Moor, die Baumwachstum in diesem Höhenbereich bereits nachwies (Vorren et al. 1993). Die dendrochronologisch analysierten Moorhölzer datieren dabei in den Zeitraum zwischen etwa 5500 und 2000 v. Chr. Das Fehlen des Auffindens älterer Hölzer liegt wohl in der Beschränkung der Suche auf die obersten $90 \mathrm{~cm}$ des
Moores begründet. Die Kulmination von Hölzern zwischen etwa 5500 und 4500 ist klimageschichtlich erklärbar: für diesen Zeitraum ist im nahe gelegenen Kaunertal eine hohe Waldgrenzlage (Nicolussi et al. 2005, Nicolussi 2009) und für den Tschierva Gletscher (Engadin) eine extreme und auch lange Rückzugsphase (Jörin et al. 2008, Nicolussi 2009) nachgewiesen. Um 5000 v. Chr. dürfte nahe der Gurgler Alm somit ein wohl lichter Zirbenwald existiert haben. Für die Holzfunde aus dem Zeitraum um 4000 v. Chr. sind ähnliche Schlüsse zu ziehen. Die Lücken der Belegung um 4300 und 3500 v. Chr. fallen hingegen mit Phasen ungünstiger Klimabedingungen beziehungsweise mit Gletschervorstößen zusammen (Nicolussi 2009).

Die jüngsten Holzfunde aus dem Moor unmittelbar bei der Gurgler Alm datieren um 2000 v. Chr. Diese Zeitmarke fällt in verschiedenen Zeitreihen zur alpinen Waldgrenzentwicklung auf und markiert das endgültige Absinken der Baumgrenze im Alpenraum auf ein im holozänen Maßstab tiefes, für die letzten vier Jahrtausende aber charakteristisches Niveau (Nicolussi et al. 2005, Reasoner und Tinner 2008). Aufgrund dieser zeitlichen Übereinstimmung ist das Fehlen von Hölzern aus dem Moor nahe der Gurgler Alm ab rund 2000 v. Chr. klimatisch und weniger nutzungsgeschichtlich zu interpretieren.

Die deutlich unterhalb der Gurgler Alm gelegenen Moorflächen erbrachten auch 


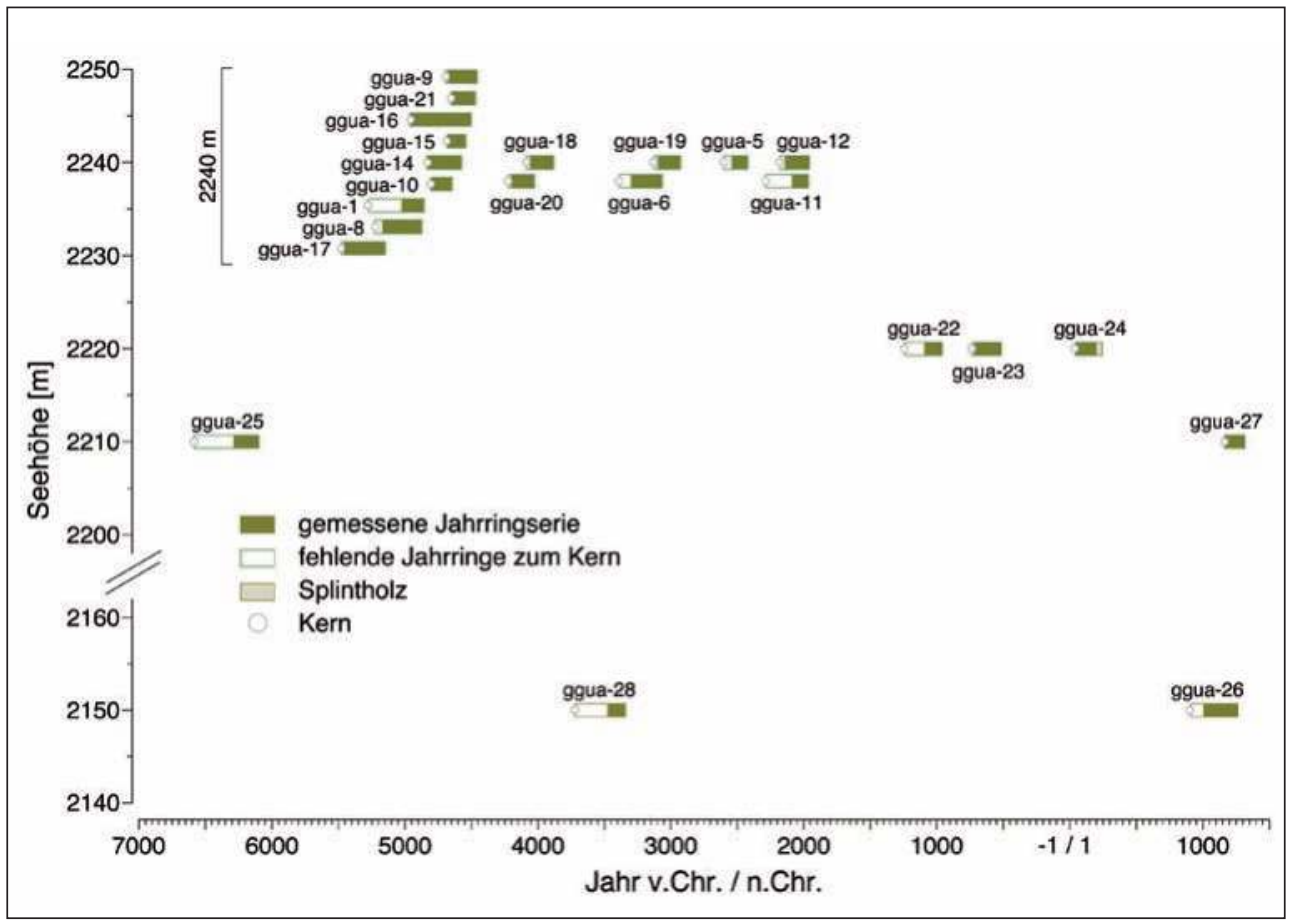

Abb. 6:

Die zeitliche Verteilung der Jahrringserien der datierten subfossilen Hölzer im Bereich der Moorflächen der Gurgler Alm, aufgetragen nach Fundorthöhen. Genannt ist jeweils die Probenbezeichnung. Für die neben der Klammer vermerkten Hölzer ist jeweils die gleiche Fundhöhe $(2240 \mathrm{~m})$ gegeben.

jüngere, in den Zeitraum nach 2000 v. Chr. datierende (zum Teil aber auch ältere) Holzfunde (Abb. 6). Die insgesamt geringe Anzahl von geborgenen Holzproben aus den letzten vier Jahrtausenden ist am ehesten durch die Erhaltungsmöglichkeiten in den befundeten, flächenmäßig meist sehr kleinen Moorarealen begründet. Die Baumgrenze selbst ist auch in diesem Zeitabschnitt des Holozäns wohl kaum je unter $2150 \mathrm{~m}$ abgesunken. Das gänzliche Fehlen von Hölzern aus den letzten 700 Jahren dürfte am ehesten wie- derum mit anthropogenen Eingriffen im Zusammenhang mit der ab dem Hochmittelalter intensiven Hochlagennutzung im innersten Gurgler Tal und weniger mit der Klimavariabilität erklärbar sein.

\section{Fazit}

Mit den hier vorgelegten Ergebnissen dendrochronologischer Auswertungen an 
Probenmaterial aus dem Raum Obergurgl wird eine vor rund 40 Jahren begonnene, damals von der Alpinen Forschungsstelle Obergurgl initiierte Forschungstradition fortgesetzt.

Die Analyse der Jahrringbreitenzuwächse von lebenden Zirben aus dem Obergurgler Zirbenwald zeigt die erwartete Übereinstimmung des Jahrringbreitenwachstums dieser Hochlagenbäume mit der Sommertemperaturentwicklung. Bemerkenswert ist, dass keine Hinweise auf ein negatives Abweichen (divergence) zwischen der Jahrringentwicklung und der Temperaturzunahme in den letzten zwei bis drei Jahrzehnten festgestellt werden konnten.

Die Analyse der zeitlichen Verteilung von subfossilen Hölzern aus Mooren im Raum von Obergurgl erbrachte unterschiedliche Ergebnisse: die Konzentration der Holzdatierungen aus dem Zirbenwaldmoor und aus den nahe gelegenen Moorflächen auf den Zeitraum zwischen etwa 100 und 1300 n. Chr. ist einerseits durch die lokale Moorentwicklung, andererseits durch verschiedene Phasen anthropogener Eingriffe in den das Moor umgebenden Wald begründbar. Demgegenüber spiegelt die zeitliche Verteilung der subfossilen Hölzer aus dem Bereich der Gurgler Alm vor allem die holozäne Waldgrenzentwicklung zumindest in Ansätzen wider.

\section{Literatur}

Auer, I., Böhm, R., Jurkovic, A., Lipa, W., Orlik, A., Potzmann, R., Schöner, W., Ungersböck, M., Matulla, C., Briffa, K., Jones, P., Efthymiadis, D., Brunetti, M., Nanni, T., Maugeri, M., Mercalli, L., Mestre, O., Moisselin, J.M., Begert, M., Müller-Westermeier, G., Kveton, V., Bochnicek, O., Stastny, P., Lapin, M., Szalai, S., Szentimrey, T., Cegnar, T., Dolinar, M., Gajic-Capka, M., Zaninovic, K., Majstorovic, Z., Nieplova, E. (2007) HISTALP—historical instrumental climatological surface time series of the Greater Alpine Region. Int. Journal of Climatology 27/1: 17-46.

Böhm, R., Jones, P. D., Hiebl, J., Brunetti, M., Frank, D., Maugeri, M. (2009) The early instrumental warm-bias: a solution for long central European temperatures series 1760-2007. Climate Change 101: 41-67.

Briffa, K.R., Jones, P. D., Bartholin, T.S., Eckstein, D., Schweingruber, F.H., Karlen, W., Zetterberg, P., Eronen, M. (1992) Fennoscandian summers from AD 500: temperature changes on short and long timescales. Climate Dynamics 7: 111119.

Briffa, K.R., Jones, P. D., Schweingruber, F.H., Karlen, W., Shiyatov, S.G. (1996) Tree ring variables as proxy-climate indicators. Problems with low-frequency signals. In: Climatic variations and forcing mechanisms of the last 2000 ye- 
ars. Jones, P.D., Bradley, R.S., Jouzel, J. (Hg.), Springer, Berlin: 9-41.

Büntgen, U., Esper, J., Frank, D.C., Nicolussi, K., Schmidhalter, M. (2005) A 1052year tree-ring proxy for Alpine summer temperatures. Climate Dynamics 25: 141-153.

Büntgen, U., Tegel, W., Nicolussi, K., McCormick, M., Frank, D., Trouet, V., Kaplan, J.O., Herzig, F., Heussner, K.-U., Wanner, H., Luterbacher, J., Esper, J. (2011) 2500 Years of European Climate Variability and Human Susceptibility. Science 331: 578-582.

Cook, E.R., Briffa, K.R., Meko, D.M., Graybill, D.A, Funkhouser, G. (1995) The segment length curse in long tree-ring chronology development for paleoclimatic studies. Holocene 5: 229-237.

D’Arrigo, R., Wilson, R., Liepert, B. and Cherubini, P. (2008) On the 'Divergence Problem' in Northern Forests: A Review of the Tree-Ring Evidence and Possible Causes. Global and Planetary Change 60: 289-305.

Eckstein, D., Aniol, R.W. (1981) Dendroclimatological reconstruction of the summer temperatures for an alpine region. In: Dickenwachstum der Bäume. Symposium Innsbruck 9.-12.9.1980. Mitteilungen der Forstlichen Bundesversuchsanstalt Wien 142: 391-298.

Esper, J., Büntgen, U., Frank, D., Pichler, T., Nicolussi, K. (2007.) Updating the Tyrol tree-ring data set. In: TRACE - Tree Rings in Archaeology, Climatology and Ecology 5. Haneca, K., Verheyen, A.,
Beeckman, H., Gärtner, H., Helle, G., Schleser, G. (Hg.): 80-84.

Frank, D., Büntgen, U., Böhm, R., Maugeri, M., Esper, J. (2007) Warmer early instrumental measurements versus colder reconstructed temperatures: shooting at a moving target. Quaternary Science Reviews 26: 3298-3310.

Giertz, V., Grießer, I. (1975) Dendrochronologie. Tiroler Heimatblätter 3: 94-100.

Jörin, U.E., Nicolussi, K., Fischer, A., Stocker, T.F., Schlüchter, C. (2008) Holocene optimum events inferred from subglacial sediments at Tschierva Glacier, Eastern Swiss Alps. Quaternary Science Reviews 27/3-4: 337-350.

Lamprecht, A. (1978) Die Beziehung zwischen Holzdichtewerten von Fichten aus subalpinen Lagen des Tirols und Witterungsdaten aus Chroniken im Zeitraum von 1370-1800 AD. Diploma Thesis. Universität Zürich.

Melvin, T. M, Briffa, K.R. (2008): A „SignalFree" approach to Dendroclimatic Standardisation. Dendrochronologia 26: 71 86.

Nicolussi, K., Lumassegger, G., Patzelt, G., Pindur, P., Schießling P. (2004) Aufbau einer holozänen Hochlagen-JahrringChronologie für die zentralen Ostalpen: Möglichkeiten und erste Ergebnisse. In: Innsbrucker Jahresbericht 2001/2002, Innsbrucker Geographische Gesellschaft (Hg.), 16: 114-136.

Nicolussi, K., Kaufmann, M., Patzelt, G., van der Plicht, J., Thurner, A. (2005) Holocene tree-line variability in the Kauner 
Valley, Central Eastern Alps, indicated by dendrochronological analysis of living trees and subfossil logs. Vegetation History and Archaeobotany 14: 221-234.

Nicolussi, K. (2009): Alpine Dendrochronologie - Untersuchungen zur Kenntnis der holozänen Umwelt- und Klimaentwicklung. In: Klimawandel in Österreich. Schmidt, R., Matulla, C., Psenner, R. (Hg.) (innsbruck university press) Alpine Space - man \& environment 6: 41-54.

Nicolussi, K., Kaufmann, M., Melvin, T.M., van der Plicht, J., Schießling, P., Thurner, A. (2009) A 9111 year long conifer tree-ring chronology for the European Alps - a base for environmental and climatic investigations. The Holocene 19: 909-920.

Oberhuber, W., Kofler, W., Pfeifer, K., Seeber, A., Gruber, A., Wieser, G. (2008) Long-term changes in tree-ring-climate relationships at Mt.Patscherkofel (Tyrol, Austria) since the mid 1980s. Trees 22: 31-40.

Reasoner, M.A., Tinner, W. (2008) Holocene treeline fluctuations. In: Encyclopedia of Paleoclimatology and Ancient Environments. Gornitz, V. (Hg.), Springer, Dordrecht: 442-446.

Rybnicek, K., Rybnickova, E. (1977) Mooruntersuchungen im Oberen Gurgltal, Ötztaler Alpen. Folia Geobot. Phytotax. Praha 12: 245-291.
Schiechtl, H.M., Stern, R. (1975) Die Zirbe (Pinus cembra L.) in den Ostalpen I. Angewandte Pflanzensoziologie 22: 84 S.

Vorren, K.-D., Morkved, B., Bortenschlager, S. (1993) Human impact on the Holocene forest line in the Central Alps. Vegetation History and Archaeobotany 2: 145-156.

\section{Verzeichnis der AutorInnen}

Kurt Nicolussi

Institut für Geographie

Universität Innsbruck

Innrain 52, 6020 Innsbruck

kurt.nicolussi@uibk.ac.at

Andrea Thurner

Institut für Geographie

Universität Innsbruck

Innrain 52, 6020 Innsbruck

andrea.thurner@uibk.ac.at 


\title{
Kapitel 7 I Zur Diversität der Flechten und Moose der subalpinen Stufe im Raum Obergurgl
}

\author{
Georg Gärtner, Wolfgang Hofbauer
}

\section{Zusammenfassung}

In der Wald- und Zwergstrauchzone im Raum Obergurgl sind Moose und Flechten wesentliche Elemente der Vegetation. Neben Boden bewohnenden Arten mit weiter ökologischer Amplitude und Verbreitung sind einige Flechten (wie Wolfsflechte oder Landkartenflechte) spezialisierte Baum- bzw. Felsbesiedler. Im Obergurgler Zirbenwald bilden mehrere häufig vorkommende Waldbodenmoose (z.B. Rotstängel, Etagenmoos u.a.) Massenbestände, im Moor dagegen dominieren Torf- und Braunmoose. Die subalpine Zwergstrauchheide mit ihrer Vielfalt an Kleinstandorten (z.B. Quellaustritte, Felsblöcke, Windkanten, Schneetälchen) weist jeweils sehr typische Moos- und Flechtengesellschaften auf, wobei besonders Feuchtezeiger (z.B. Lebermoose der Gattung Scapania) erwähnenswert sind. Eine lokale Besonderheit bilden die Massenbestände diverser terrestrischer Strauchflechten (z. B. Flavocetraria-, Cladonia- und Alectoria-Arten) in der Gurgler Heide. Seltene Substratspezialisten wie Splachnaceen unter den Laubmoosen kommen auf Tierexkrementen oder in Schneetälchen (z.B. Safranflechte und Norwegisches Haarmützenmoos) vor.

\section{Abstract}

Lichens and mosses are a prominent part of the coniferous belt and the subalpine dwarf shrub zone around Obergurgl. More common and widely distributed species of terrestrial mosses and lichens can be found in the famous Zirbenwald (forest of Pinus cembra), while specialized types are covering tree bark or rock surfaces (e.g. the macrolichen Letharia vulpina). In acidic fens like the "Zirbenwaldmoor" Sphagnum mosses and brown mosses (e.g. Warnstorfia, Straminergon and others) are dominating. The locality "Gurgler Heide" in the subalpine zone is characterized by terrestrial fruticose lichens (e.g. Flavocetraria, Alectoria) and reindeer lichens (Cladonia species). Specific habitats for mosses are lumps of herbivore dung covered by Splachnaceae or wet soils in late-lying snow beds where the arctic-alpine Polytrichastrum sexangulare can be found together with the foliose lichen Solorina crocea. 
Einleitung

Klima, Gesteinsuntergrund und menschliche Siedlungstätigkeit prägen die heutige Pflanzendecke des inneren Ötztales und den Raum Obergurgl. Während die waldfreien Areale - also Gletscher, Felsen, Moränen, alpine Rasen, Bergmähder, Weiden und Zwergstrauchheiden - etwa $85 \%$ der Gesamtfläche einnehmen, entfallen nur knapp 7\% auf Wälder. Vorwiegend sind es Lärchen-Fichtenwälder, Lärchen-Zirbenwälder und reine Zirbenbestände (Larici-Piceetum subalpinum, Lariceto-Pinetum cembrae, Pinetum cembrae, Pitschmann et al. 1980). Sie beschränken sich auf vorwiegend hangseitige Bestände an den Talflanken von Obergurgl talauswärts beziehungsweise auf einen der schönsten Zirbenwälder in den Ostalpen südlich des Ortes Obergurgl (s. Kap. 4). Wie in den meisten Gebieten der Alpen sind die Waldbestände durch die Jahrtausende lange Bewirtschaftung sowie durch Rodungen für den Wintertourismus stark verkleinert worden. Umso mehr gilt es die Restbestände und deren Schutzwirkung für den Siedlungs- und Wirtschaftsraum zu bewahren.
Forschungsgeschichte

Die Waldgrenze, heute anthropogen bedingt bei ca. 2000 bis $2100 \mathrm{~m}$, wäre unter natürlichen Bedingungen höher (Zirbenvorkommen im Bereich des Schönwieskopfes bei $2300 \mathrm{~m}$ ). Ihre vegetationsgeschichtliche Entwicklung und Ökophysiologie ist seit langem Gegenstand wissenschaftlicher Forschung und intensiv dokumentiert (u.a. Tranquillini 1979, 2000, Bortenschlager 1984, 2000, 2010). Über die Zirbenwälder sowie über die Vegetation und Ökologie der subalpinen Stufe im inneren Ötztal liegen ausführliche Langzeitstudien vor (u.a. Schiechtl 1965 - mit Vegetationskarte 1:37 500 des Gurgler Tales, weiters die Sammelbände in den Mitt. d. Forstl. Bundesversuchsanstalt 1959, 1965 und 1967). Eine Karte der aktuellen Vegetation im Maßstab 1: 100000 publizierten Pitschmann et al. 1980, weitere relevante Literatur zur subalpinen Zone Obergurgls ist bei Erschbamer (2000) sowie bei Koch \& Erschbamer (2010) angeführt.

Die naturwissenschaftliche Erforschung des Raumes um Obergurgl, welche im engen Zusammenhang mit der gletscherkundlichen Forschung verlief, ist bereits in Band I der Reihe ausführlich dargestellt (Gärtner 2010), wobei auch die bisher vorliegenden Untersuchungen über Verbreitung und Vorkommen diverser Kryptogamengruppen (Algen, Flechten, Moose) zitiert sind. Neben Artenlisten 
von Exkursionen (vergl. Hofmann et al. 1988) sind es vor allem punktuelle floristische Notizen, die ein Bild der reichen Diversität der niederen Pflanzen vermitteln und mehrere, besonders für Exkursionen geeignete Lokalitäten herausheben.

\section{Der Obergurgler Zirbenwald}

Seit 1963 als Naturdenkmal ausgewiesen und heute ein Teil des UNESCOBiosphärenparks Gurgler Kamm bedeckt der Zirbenwald (Abb. 1) südlich von
Obergurgl eine Fläche von 20 ha zwischen $1950 \mathrm{~m}$ bis $2100 \mathrm{~m}$ Seehöhe. Die Bestandesstruktur ist einerseits durch sehr alte Baumriesen (mehr als 300 Jahre alt) und daneben reichen Jungwuchs gekennzeichnet. Dies wird auf einen Waldbrand um 1900 zurückgeführt. Neben epiphytischen und Boden bewohnenden Flechten und Moosen sind einige steil stehende, sonnig bis halbschattige Felsblöcke mit epilithischen Flechten bemerkenswert.

Unter den Boden bewohnenden Flechten sind eine Reihe von Großflechten (Makrolichenes) zu erwähnen, darunter Becherflechten beziehungsweise Rentierflechten (Cladonia) wie Cladonia ar-

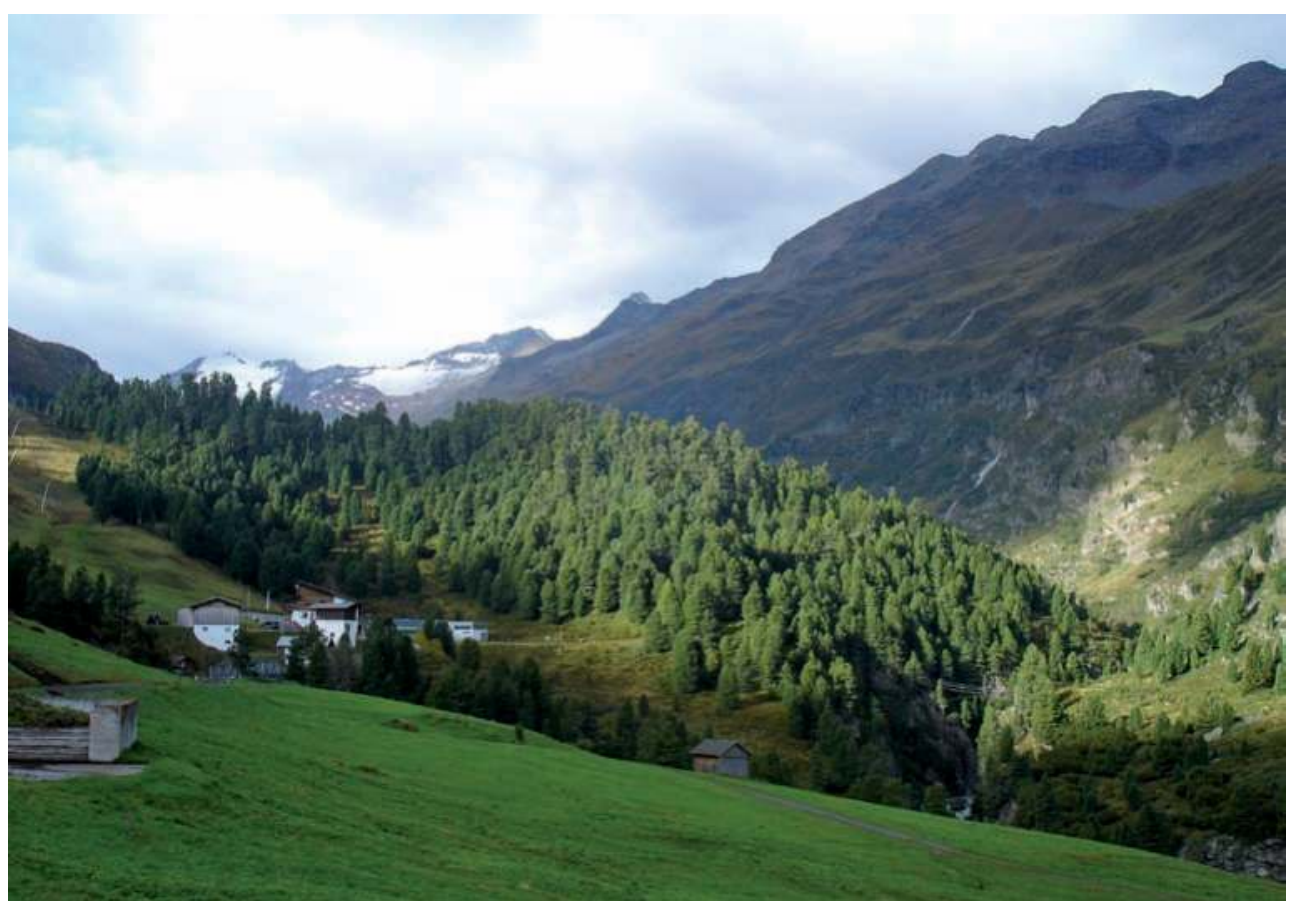

Abb. 1:

Naturdenkmal Obergurgler Zirbenwald (Foto: Georg Gärtner) 


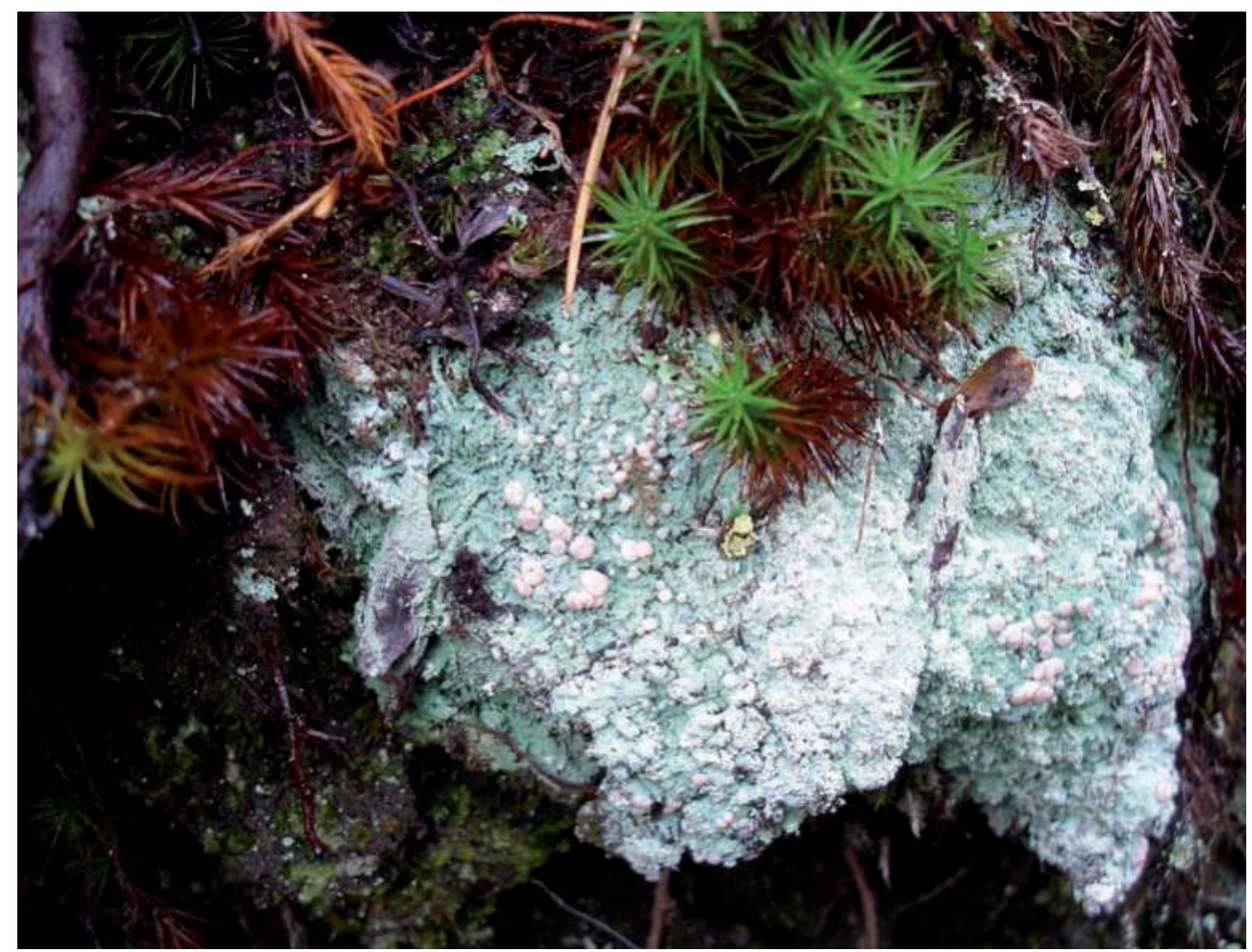

Abb. 2:

Heideflechte (Icmadophila ericetorum) und Schönes Haarmützenmoos (Polytrichum = Polytrichastrum formosum) auf feuchtem Torfsubstrat im Zirbenbestand (Foto: Georg Gärtner)

buscula s.l., Cl. bellidiflora, $\mathrm{Cl}$. cenotea, $\mathrm{Cl}$. cornuta, Cl. furcata, Cl. gracilis, Cl. macilenta subsp. macilenta, $\mathrm{Cl}$. macroceras, $\mathrm{Cl}$. pleurota, $\mathrm{Cl}$. pyxidata, Cl. rangiferina, $\mathrm{Cl}$. squamosa, Cl. stellaris, Cl. sulphurina u.a. Auf vermoderndem Holz und vertorften Moospolstern an sehr schattigen, feuchten Stellen fallen auf grünem krustigem Lager die fleischroten Fruchtkörper der Heideflechte Icmadophila ericetorum (Abb. 2) auf, während zwischen Moosen und unter Grasbüscheln die großen breitlappigen Lager (Thalli) der Apfelflechte (Peltigera aphthosa mit feucht apfelgrün erscheinendem Lager und oberseits schwarzen Warzen $=$ Cephalodien aus Cyanobakterien, Abb. 3) im Zirbenwald nicht selten sind. Eine verwandte Art, Peltigera leucophlebia, kommt ebenfalls vor. Ihre Lagerunterseite ist hell mit dunklen Adern.

Nicht nur im Zirbenwald, sondern in der gesamten Zwergstrauchzone bis in die nivale Stufe kommt das Isländische Moos (Cetraria islandica) vor, eine Strauchflechte mit sehr weiter ökologischer Amplitude und arktisch-alpiner Verbreitung. Ihre 


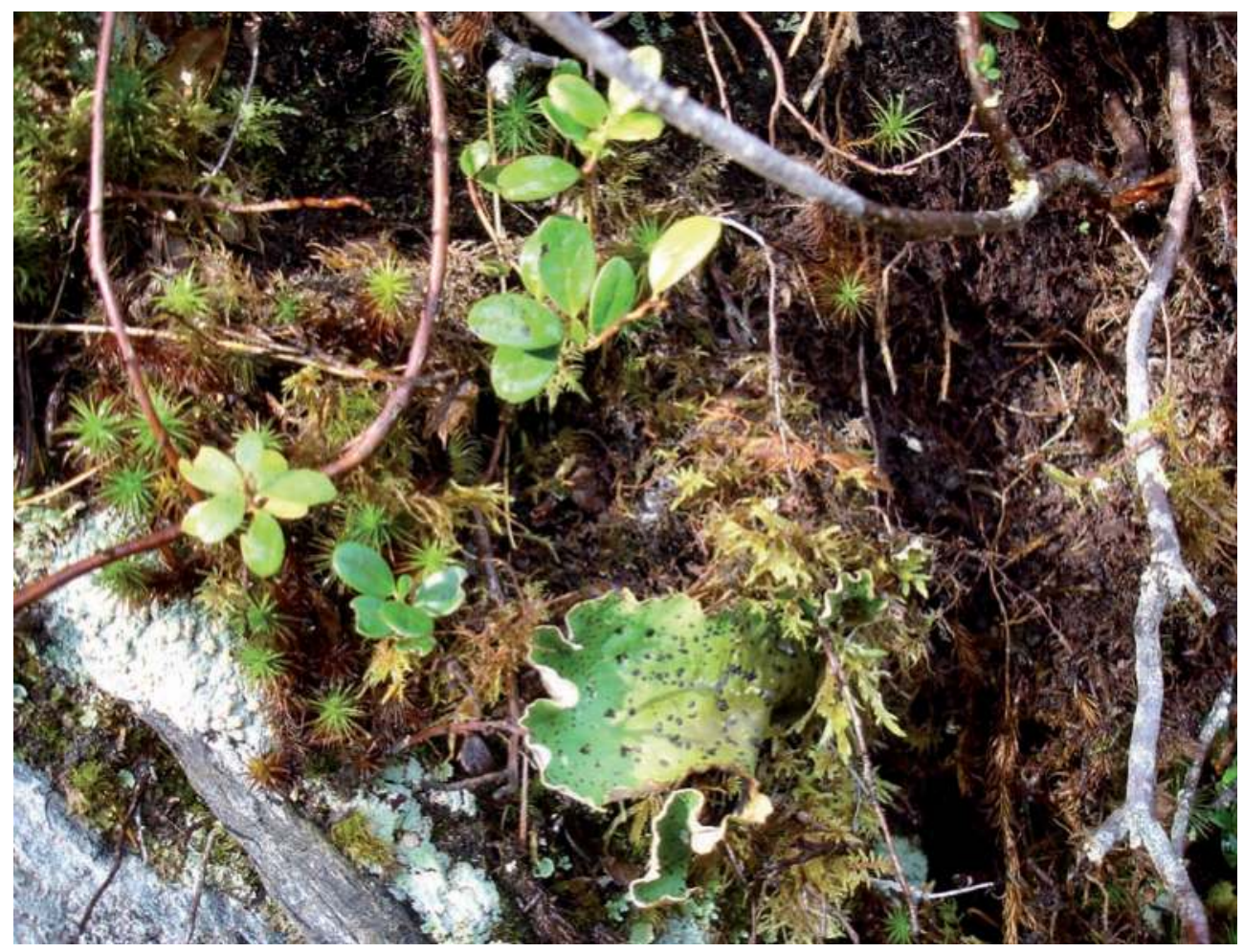

Abb. 3:

Peltigera aphthosa (Apfel- oder Schildflechte mit schwarzen Cephalodien an der Thallusoberseite) vergesellschaftet mit Pleurozium schreberi und Polytrichum (Polytrichastrum) formosum im Unterwuchs unter Rhododendron ferrugineum und Vaccinium vitis-idaea an der Waldgrenze (Foto: Georg Gärtner)

aufrechten, braun bis grünlich gefärbten Thalluslappen können bis über $10 \mathrm{~cm}$ hoch werden und weisen unterseits weiße Flecken (= Aufbrüche der Thallusrinde, Pseudocyphellen genannt) auf. Sie dienen der Durchlüftung. Die Schleimstoffe des Isländisch Moos sind seit langem in der Volksmedizin als Hustenmittel gebräuchlich, aber auch wirksamer Bestandteil von Hustenpastillen.

Die Moosdecke im Zirbenwald ist artenreich. Einige der häufigsten Waldboden- moose, wie Etagenmoos (Hylocomium splendens), Rotstängel (Pleurozium schreberi), Besen-Gabelzahnmoos (Dicranum scoparium), Runzelbruder (Rhytidiadelphus triquetrus) oder Haarmützenmoose (Polytrichum (=Polytrichastrum) formosum, $P$. commune) gemeinsam mit Lebermoosen wie Muschelmoos (Plagiochila asplenioides) oder Kammkelchmoos (Lophocolea bidentata s.1.) sind allgemein verbreitet. Sie steigen auch weit über die Waldgrenze in die Zwergstrauchheiden. 


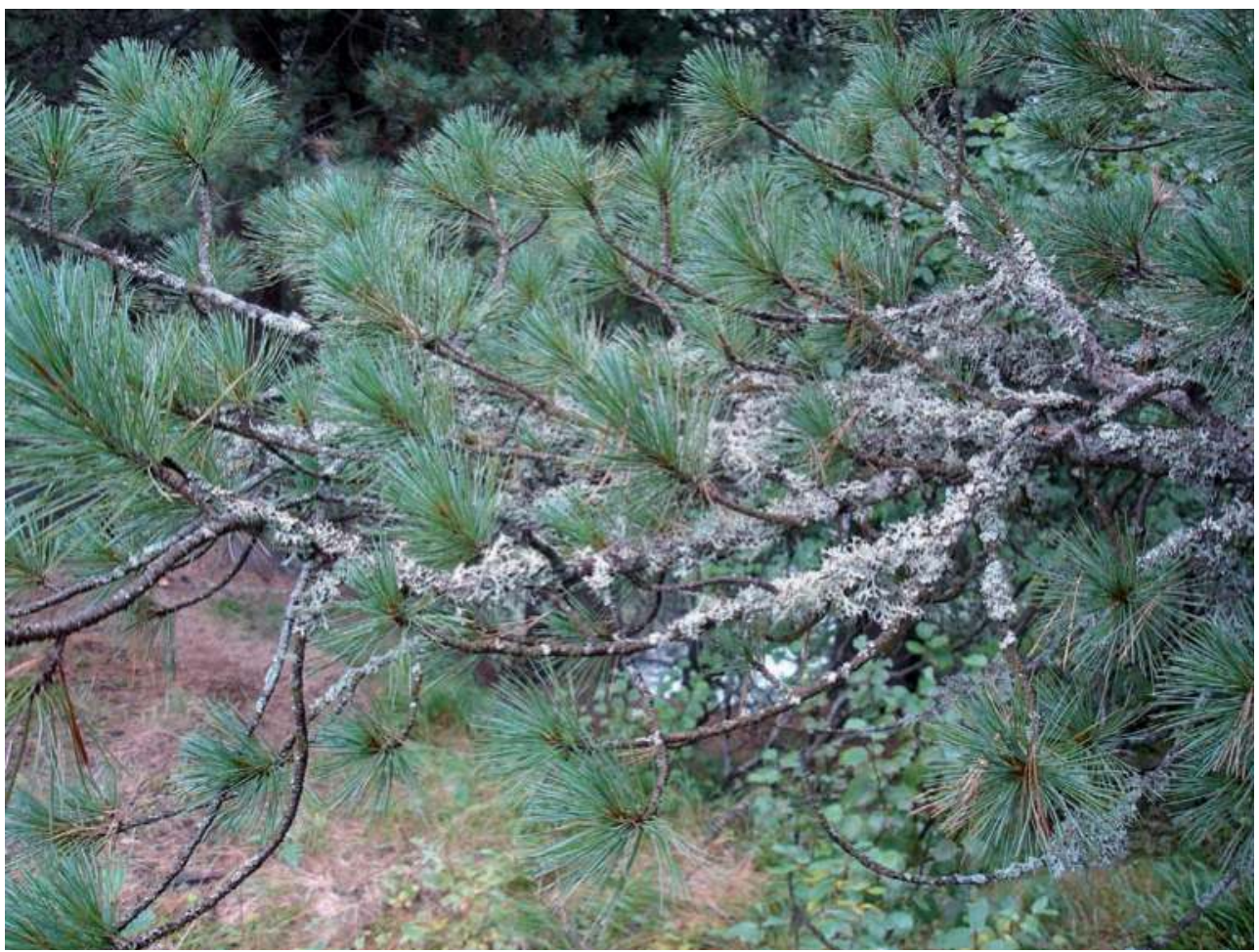

Abb. 4:

Massenbestände von Pseuevernia furfuracea im Zirbenwald (Foto: Georg Gärtner)

An feuchteren Stellen ist gelegentlich ein Torfmoos, Sphagnum quinquefarium, anzutreffen. An Stammbasen ist das euryöke und formenreiche Zypressenmoos (Hypnum cupressiforme) ebenfalls allgegenwärtig.

Epiphytische, also auf Baumrinden siedelnde Flechten sind im Zirbenwald mit einigen charakteristischen und auch ohne Lupe leicht anzusprechenden makrolichenen Arten vertreten. Dichte Massenbestände auf Zirbenzweigen und Lärchen (wie z. B. am Ochsenkopf) bildet die graue Pseudevernia furfuracea (Abb. 4), oft gemeinsam mit Hypogymnia physodes, $H$. bitteri und Bartflechten der Gattung Usnea (U. filipendula u.a.). Im LärchenZirbenwald des Ochsenkopfes, vor allem an der Nordseite gegen Poschach, findet man Pseudevernia furfuracea häufig mit Fruchtkörpern. Zu den lichenologischen Kostbarkeiten zählt das immer noch reiche Vorkommen der giftigen Wolfsflechte (Letharia vulpina), auf alten Zirben- und Lärchenstämmen. Ihre leuchtend zitronen- bis dunkelgelben strauchigen, starren Lager sind reichlich mit feinen zylindrischen Isidien (=stiftförmige asexuelle 


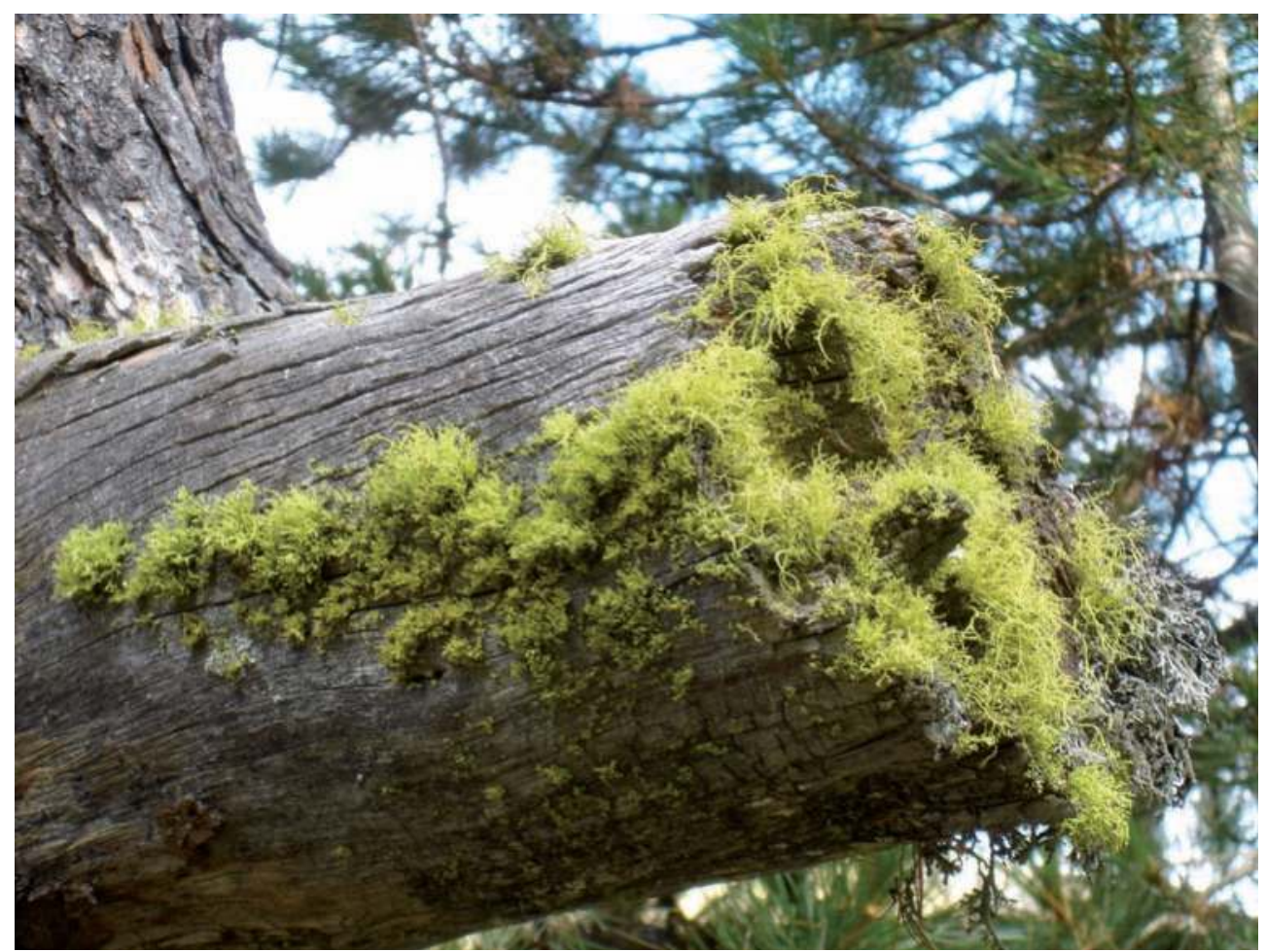

Abb. 5:

Wolfsflechte (Letharia vulpina) auf alter Zirbe (Foto: Georg Gärtner)

Vermehrungskörper) besetzt. Apothezien bei Letharia wurden im Obergurgler Zirbenwald noch nicht gefunden, sie sind sehr selten. Die Giftigkeit der Wolfsflechte beruht auf dem Gehalt an gelber Vulpinsäure, welche in Nordeuropa als Giftstoff für Wolfsköder dient. An der Stammbasis der Bäume und an dünnen Zweigen von Jungtrieben in Bodennähe fällt eine gelbgrüne Blattflechte - Vulpicida pinastri - mit zitronengelben, mehligen Rändern (= Bortensorale) auf. In diesen bildet die Flechte vegetative Vermehrungseinheiten (= Soredien), welche Algen und Pilzhy- phen enthalten und gemeinsam verbreitet werden. Vulpicida pinastri erträgt längere Schneebedeckung, ist aber frostempfindlich. Sie gilt als "Schneepegelflechte“ (Abb. 6) und kommt häufig gemeinsam mit rosettig wachsenden, nur bis $3 \mathrm{~cm}$ großen Blattflechten der Gattung Parmeliopsis (P. ambigua gelblichgrün, P. hyperopta grau) vor.

Die epiphytische Moosflora ist im Gebiet im Verhältnis zu den Flechten eher artenarm, neben Hypnum cupressiforme ist noch das Fiederchen-Lebermoos (Ptilidium pulcherrimum) erwähnenswert. Seine 


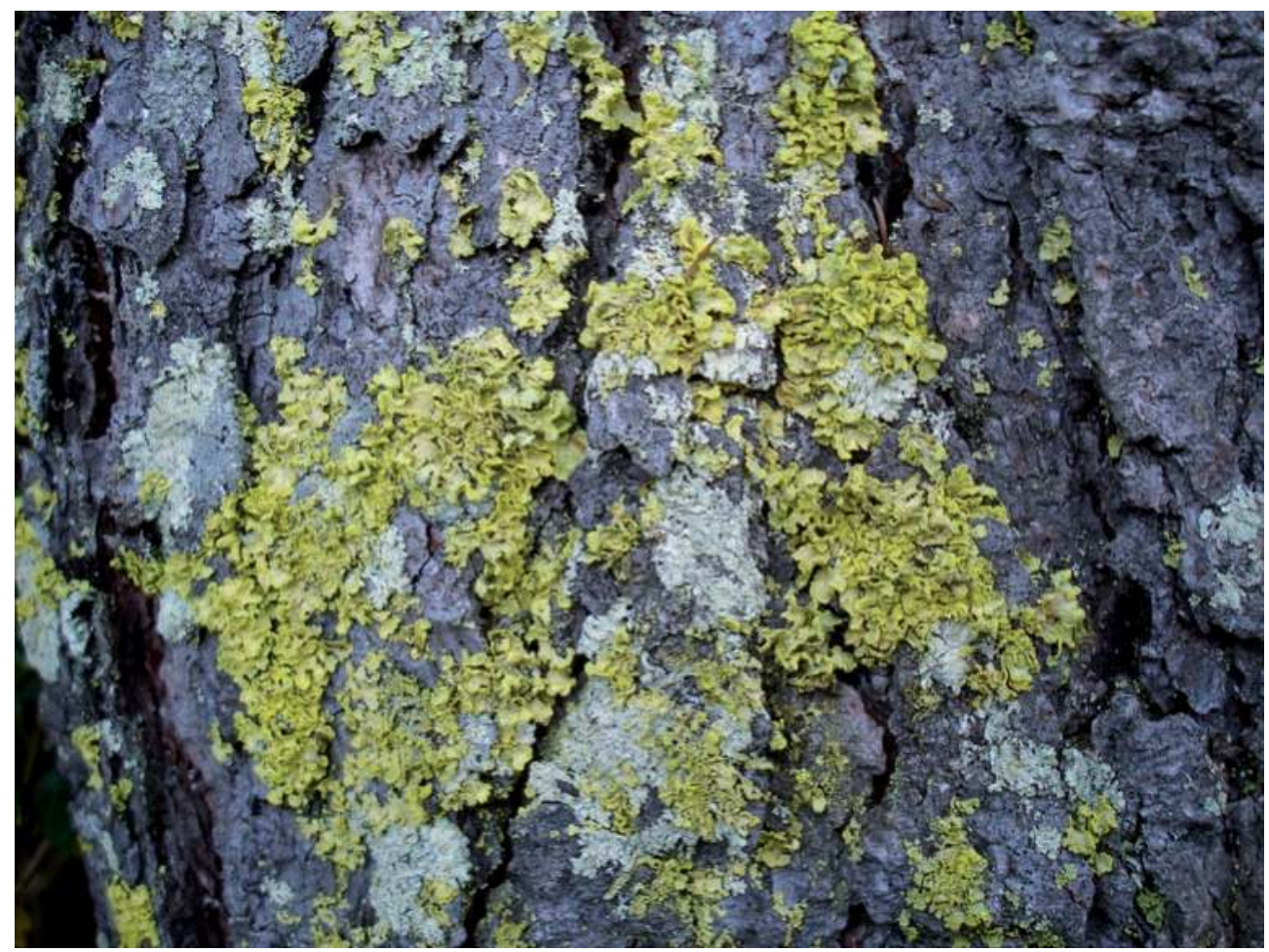

Abb. 6:

Schneepegelflechten (gelb - Vulpicida pinastri, graugrünlich - Parmeliopsis ambigua) auf Lärchenborke (Foto: Georg Gärtner)

Blättchen sind gelappt und mit feinen Wimpern versehen.

Ein spezielles Substrat für viele Moose und Flechten bildet Totholz. An alten toten und bereits borkenfreien Zirbenstämmen sind Xylographa-Arten mit einer guten Lupe sicher anzusprechen. Ihr Lager ist dünn, unscheinbar und im Holz entwickelt, nur die schwarzen Fruchtkörper sind an der Oberfläche erkennbar, wobei diese bei Xylographa parallela (Abb. 7) länglich und parallel zu den Holzfasern angeordnet sind. Eine zweite
Art, $X$. vitiligo, ist ebenfalls auf toten entrindeten Zirbenstämmen in Obergurgl nachgewiesen (Hofmann et al. 1988). Von den Moosen ist auf morschem, vermoderndem Holz unter anderem das Vierzahn- oder Georgsmoos (Tetraphis pellucida) zu finden, mit seinen typischen Kapseln, deren Peristom aus lediglich vier spitzen Zähnen besteht. In der vegetativen Form bildet das Moos am Ende der Stämmchen kleine Brutbecher aus, die Brutkörper werden durch Regentropfen herausgeschleudert. Der von Georgs- 


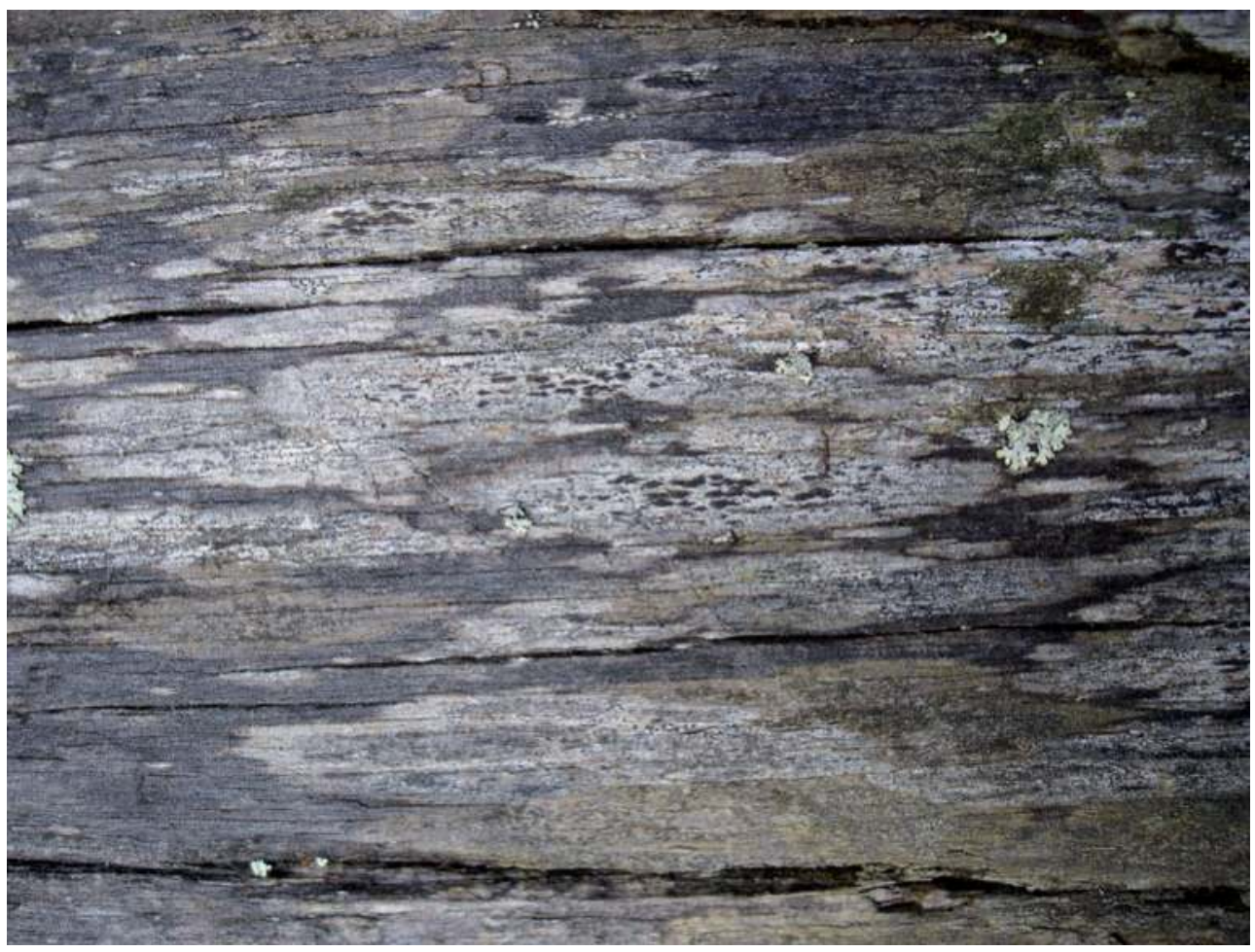

Abb. 7:

Xylographa parallela mit schwarzen, parallel zur Holzfaser angeordneten Fruchtkörpern und junges Lager von Parmeliopsis ambigua auf Totholz (Foto: Georg Gärtner)

moos dominierte Moosverein wird auch als Lepidozio-Tetraphidetum pellucidae angesprochen. Der Name deutet bereits darauf hin, dass auf morschen Stämmen auch das Kriechende Schuppenzweig-Lebermoos (Lepidozia reptans) häufig ist, ein hübsches regelmäßig gefiedertes Lebermoos mit viergeteilten Blättchen.

Der Obergurgler Zirbenwald ist durch einen 2,1 km langen Rundweg gut erschlossen. Entlang des Steiges sind an senkrecht gestellten Felsformationen eine Reihe bemerkenswerter epilithischer Flechten zu sehen, darunter Nabelflechten der Gattung Umbilicaria: an halbschattigen etwas sickerfeuchten Vertikalflächen Umbilicaria hirsuta, an eher besonnten Stellen die variable $U$. cylindrica, an niedrigen, bodennahen und länger schneebedeckten Felsblöcken auch die Rußflechte, $U$. deusta und andere. Gesteinsblöcke an lichtreichen Stellen bis weit hinauf in die alpine Stufe besiedeln Blattflechten wie die blaugraue, stark isidiöse Parmelia saxatilis (diese kommt auch auf sauren Borken vor!) und Krustenflechten. Am 


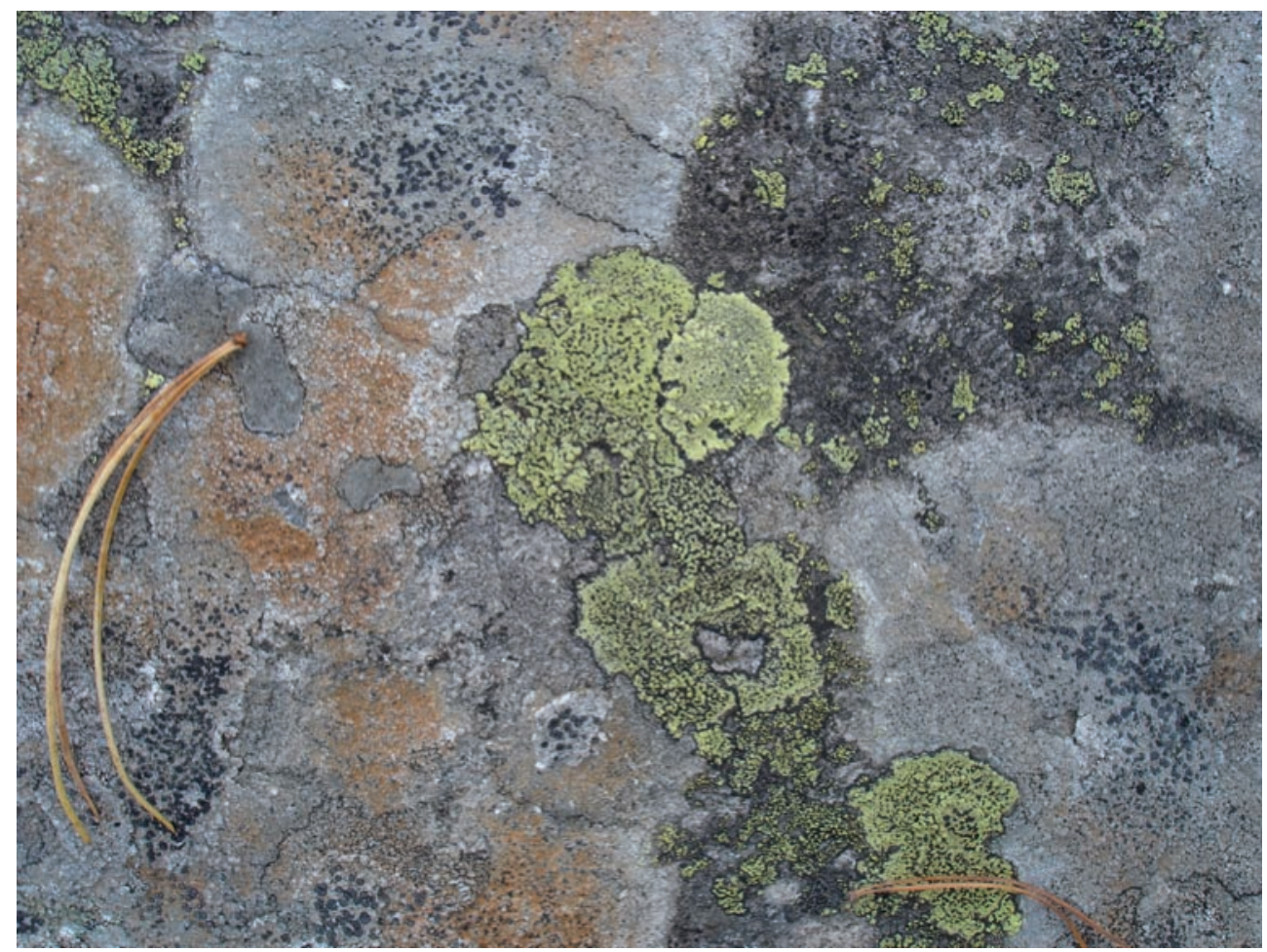

Abb. 8:

Epilithische Krusten von Rhizocarpon geographicum (gelbgrün) und Lecidea-Arten (Foto: Georg Gärtner)

bekanntesten sind die gelbgrünen Landkartenflechten der Gattung Rhizocarpon (Rhizocarpon geographicum mit mehreren Unterarten, Abb. 8). Daneben treten großflächige Lager verschiedener, schwer im Gelände ansprechbarer Krusten von Lecidea-Arten (Abb. 8) auf, wie Lecidea lithophila (häufig rostfarben) oder L. lapicida (eher hellgrau, aber auch teilweise rostfarben). Lecidea-Arten sind meist nur chemisch beziehungsweise an den Sporen und Fruchtkörperstrukturen zu unterscheiden.
An lichtoffenen Steil- und Überhangsflächen von der Waldgrenze bis in höchste Lagen siedelt die arktisch-alpine Blutaugenflechte, Ophioparma ventosa ( $=\mathrm{Ha}^{-}$ ematomma ventosum, Abb. 9). Ihr dickes krustiges Lager ist gelbgrün bis graugrün und trägt meist reichlich Fruchtkörper mit blutroter Scheibe (Name! Mit Kalilauge reagiert der Farbstoff der Apothezienscheibe blau). An solchen Vertikalflächen ist neben den großflächigen, schwarzen Thalli von Melanelia ( $=\mathrm{Ce}$ traria) hepatizon eine weitere arktisch- 


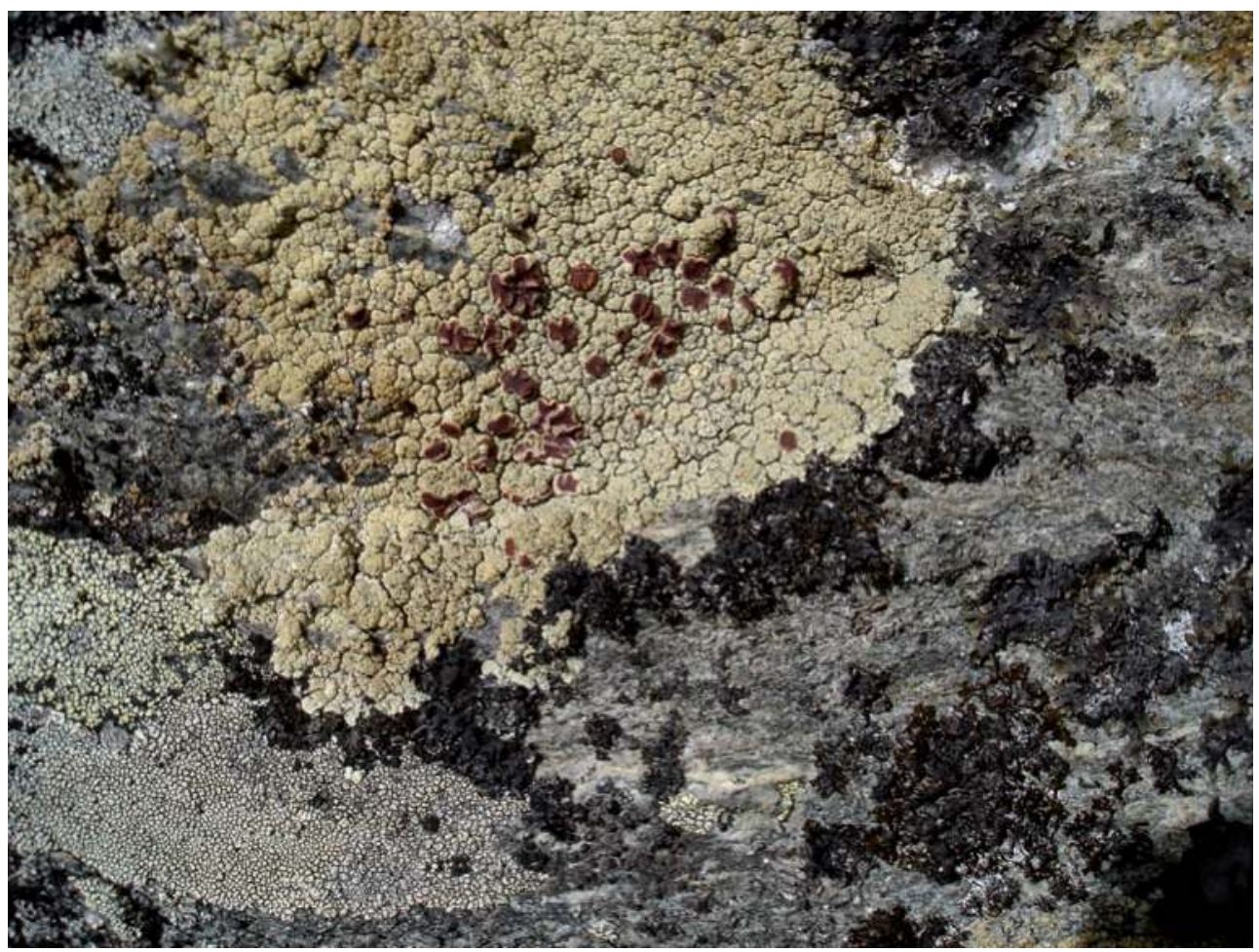

Abb. 9:

Epilithische Krusten- und Strauchflechten (Blutaugenflechte, Ophioparma ventosa, Dimelaena oreina unten links und Melanelia hepatizon, Mitte) auf Vertikalfelsflächen im Zirbenwald (Foto: Georg Gärtner)

alpine Krustenflechte Dimelaena oreina an eher sonnigeren Stellen nicht selten. Freistehende Felsen bieten vielen Vögeln geeignete Aussichts- und Ruheplätze. An solchen mit Vogelkot gedüngten Felsblöcken siedelt eine besonders bemerkenswerte Flechtengemeinschaft, darunter die blassgrünlich-gelbe Strauchflechte Ramalina capitata mit bis $3 \mathrm{~cm}$ hohen, bandförmigen, starren Lagerabschnitten und deutlichen endständigen, fast kugeligen Soralen (Kopfsorale, bilden Soredien zur vegetativen Fortpflanzung).
Fels besiedelnde Moose

Unter den Moosen kommt an schattigen, feuchten Felsen besonders in $\mathrm{Ni}$ schen und kleinen Überhängen Hallers Apfelmoos (Bartramia halleriana) vor, während an trockenen, stark besonnten Horizontalflächen trockenresistente Arten wie Hedwigsmoos (Hedwigia ciliata) mit weißen Glashaaren an den Blättern sowie einige kissenförmige schwarzgrüne Grimmiaceen, wie diverse Kissenmoo- 
se (Grimmia-Arten), das polsterförmige Siebzahnmoos (Coscinodon cribrosus) und Spalthütchenmoose (Schistidium-Arten) anzutreffen sind. In Spalten finden sich weiters Straffblättriges Apfelmoos (Bartramia ithiphylla) und Eibenblättriges Doppelblattmoos (Diplophyllum taxifolium). Je nach der vorherrschenden Art werden hier verschiedene Moosvereine gegliedert, allen voran das Hedwigietum ciliatae an den ausgesetzten Stellen. An gelegentlich überrieselten Stellen finden sich verschiedene Arten aus der Artengruppe des Ungleichästigen Zackenmützenmooses (Racomitrium heterostichum agg. = Bucklandiella heterosticha agg.). Das Spitzblättrige Blindmoos (Blindia acuta), Charakterart des Blindietum acutae, toleriert überrieselte Standorte und kommt daher z.B. häufig auf Blockoberflächen, an Bachrändern und an Wasserfällen vor, manchmal untergetaucht. Dadurch leitet dieses Laubmoos zu den eigentlichen Moosen der Fließgewässer, in dieser Höhenlage vor allem von Hygrohypnum molle dominiert, sowie zu den Sumpfstandorten über.

Eine klassische Lokalität im Obergurgler Zirbenwald ist das Moor im Zirbenwald, ein kleines Durchströmungsmoor (in einem ehemaligen Toteisloch) mit reicher Cyperaceen- und Torfmoosvegetation.

An Moosen sind Sphagnum-Arten (Torfmoose, z. B. Sphagnum magellanicum) und Braunmoose - Warnstorfia exannulata = Drepanocladus exannulatus, Straminergon stramineum $=$ Calliergon str. sowie Polytrichum strictum - charakteristisch. Während das mittlere Torfmoos (Sphagnum magellanicum, Leitart der Hochmoorbulten-Gesellschaften, Oxycocco-Sphagnetea) abwechselnd mit Braunmoosen Bereiche dominiert, die über das Grundwasser reichen, finden sich in den Schlenken besonders flutende Formen aus der Spießtorfmoos-Artengruppe (Sphagnum cuspidatum-Gruppe). Viele Vertreter der bisher in der Waldstufe genannten Moos- und Flechtenarten steigen weit in die subalpine Zone der Zwergstrauchheide empor. Eine klassische und wegen ihrer artenreichen Flechtenvegetation berühmte Lokalität ist die Gurgler Heide (Abb. 10). Sie erstreckt sich etwa in einer Höhe zwischen 2200 und $2400 \mathrm{~m}$ unterhalb des Roßkars zwischen Gaisberg- und Ferwalltal. An exponierten, windgefegten und im Winter schneefreien Kuppen mit ausgeprägten Beständen der Gämsheide (Loiseleuria procumbens) bedecken teppich- bis polsterartig eine Reihe spezialisierter Strauchflechten den Boden. Neben der weißen "Totengebeinsflechte“ oder Wurmflechte (Thamnolia vermicularis), die keine Fruchtkörper ausbildet, sind Flavocetraria nivalis, F. cucullata, Alectoria ochroleuca und A. nigricans, Cetraria islandica, $C$ ericetorum, Cladonia uncialis, Cl. stellaris, Cl. rangiferina sowie Stereocaulon alpinum häufig zu finden. Für weitere Arten sei auf die Liste in Hofmann et al. (1988) verwiesen. 


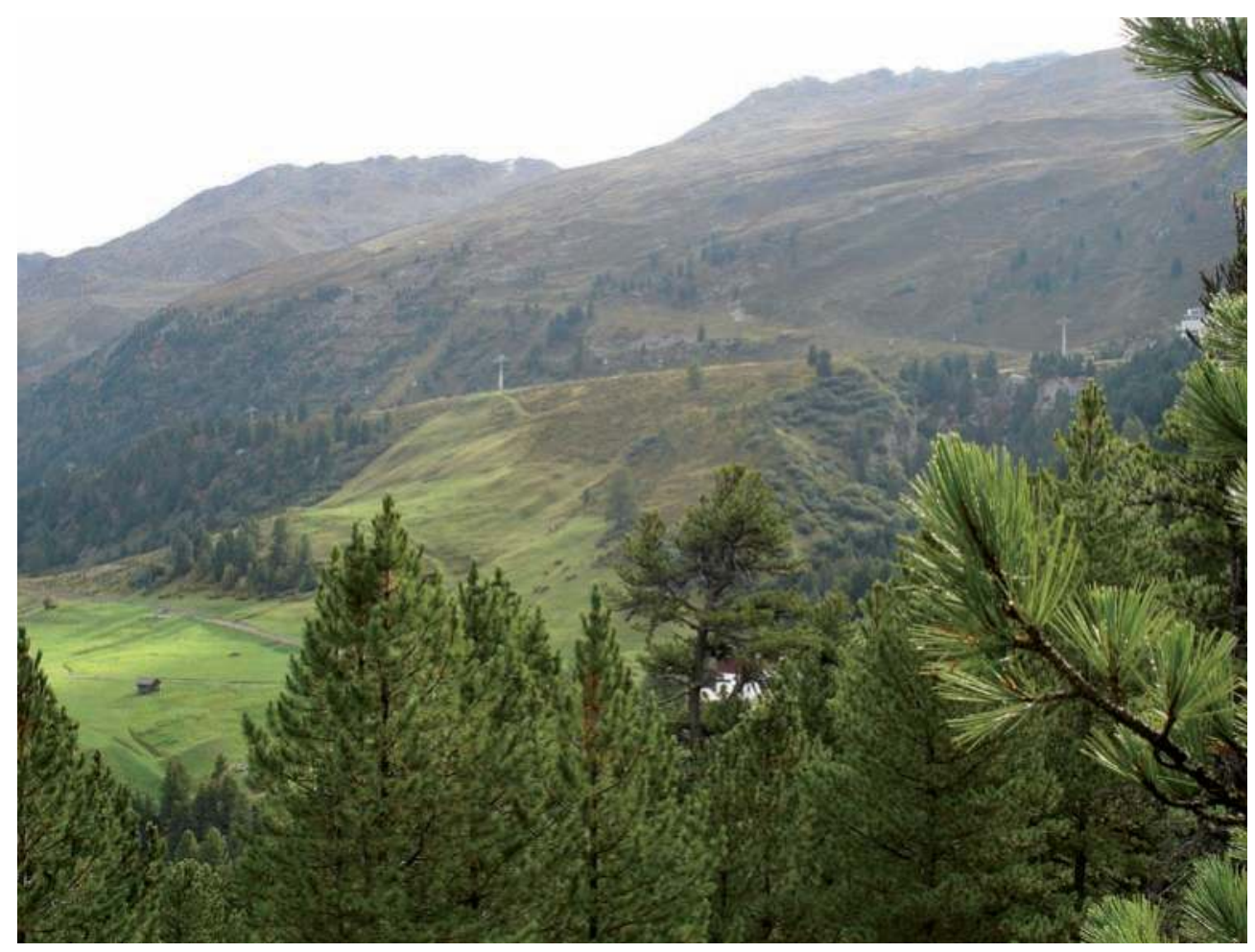

Abb. 10:

Die Gurgler Heide an der Waldgrenze, mit artenreichem Flechtenbestand in der Zwergstrauchheide (Foto: Georg Gärtner)

Unter den Moosen der subalpinen Stufe kommen zahlreiche Arten des ZirbenLärchenwaldes vor, die zum Teil bis weit in die alpine Zone vorstoßen (wie Rhytidium rugosum, Tortula ruralis oder auch das Lebermoos Ptilidium ciliare, die in den Alpen bis gegen $3000 \mathrm{~m}$ hinaufsteigen). An und in Quellbächen und kleinen Rinnsalen entwickeln sich neben dem Quellmoos Philonotis seriata (in kalkarmen alpinen Quellfluren mit Cratoneuron commutatum bzw. Palustriella commutata das Cratoneuro-Philonotidetum seriatae bildend, Sauer 2001) oft auffällige schwarzrötliche Decken eines Lebermooses (Abb. 12, Scapania undulata zusammen mit Scapania uliginosa). Bryum pseudotriquetrum, Dicranella palustris (Dichodontium palustre), Philonotis seriata und Warnstorfia exannulata sind charakteristisch für das an quelligen Stellen ausgebildete Blindio-Scapanietum uliginosae (Philippi 2005). Kalkreichere Sickerwasser-Stellen sind durch das Starknervmoos (Cratoneuron commutatum $=$ Palustriella commutata) charakterisiert. Neben Bryum 


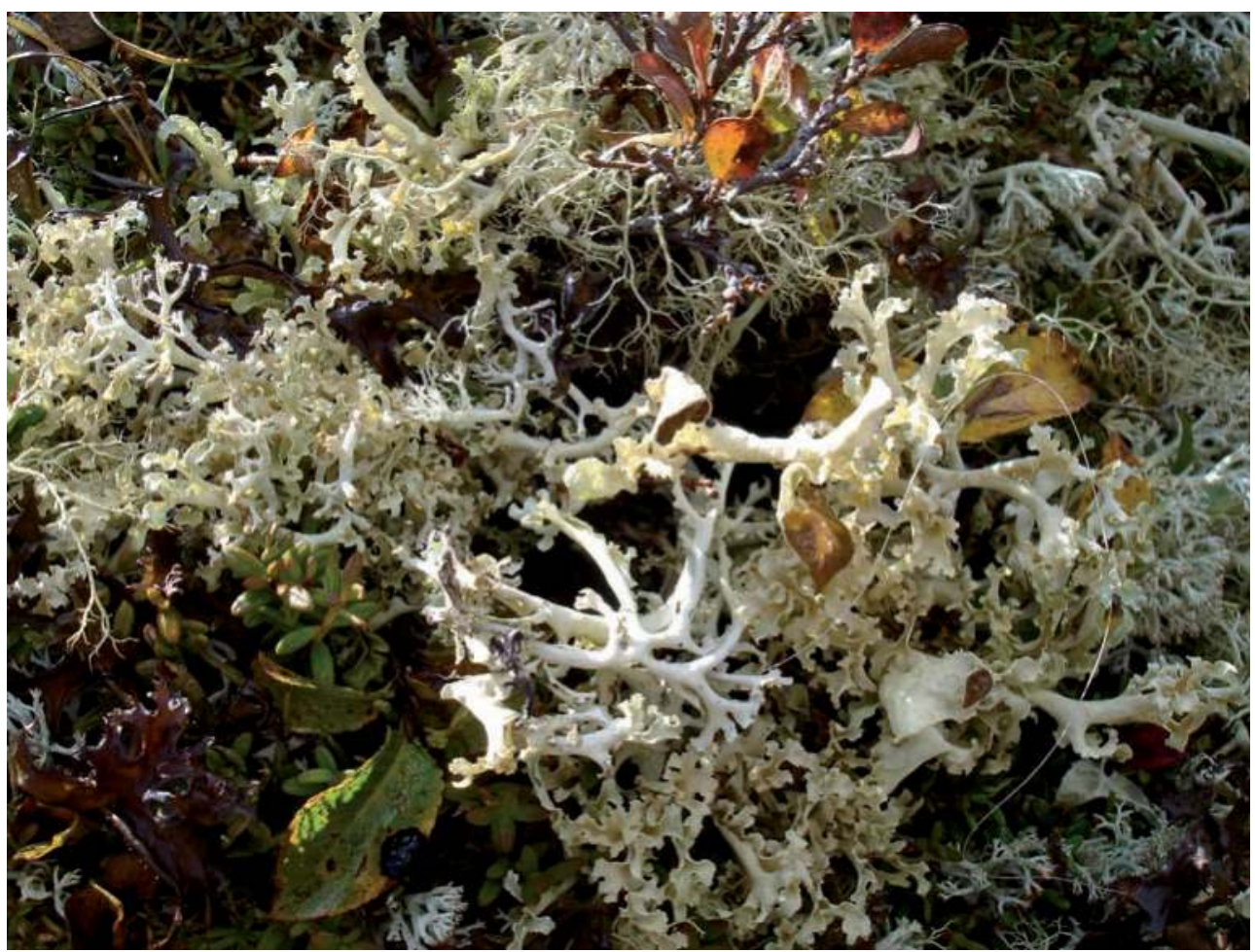

Abb. 11:

Windkantenflechten mit Alectoria ochroleuca (oben), fruchtender Flavocetraria cucullata (Mitte) sowie Cetraria islandica und Cladonia arbuscula in der Gurgler Heide (Foto: Georg Gärtner)

schleicheri sind typische Begleiter: Aneura pinguis und Campylium stellatum (Nebel 2001). In beweideten Flächen kommen auf Exkrementen diverser Tiere seltene Substratspezialisten wie Splachnum sphaericum (=ovatum), Tetraplodon urceolatus und T. mnioides vor. Ihre Sporen werden von Dungfliegen oder -käfern verbreitet, die von einem Duftstoff aus dem bauchigen Hals der Sporenkapseln angelockt werden.

Kleine Felsblöcke in der Zwergstrauchheide werden häufig von kleinen, dunkel- grün bis schwarzgrünen Polstern xerophiler Laubmoose aus der Gattung Grimmia beziehungsweise von Vertretern der Klaffmoose (Andreaea rupestris u.a.) besiedelt. Eine spezielle Moos- und Flechtenflora besiedelt sogenannte „Schneeböden“ oder „Schneetälchen“, also Stellen mit langer Schneebedeckung und starker Bodenfeuchte. Neben der bis handtellergroßen Safranflechte (Solorina crocea) mit orangeroter Lagerunterseite und braunen Fruchtkörpern dominieren Moose, wie das Norwegische Haarmützenmoos (Po- 


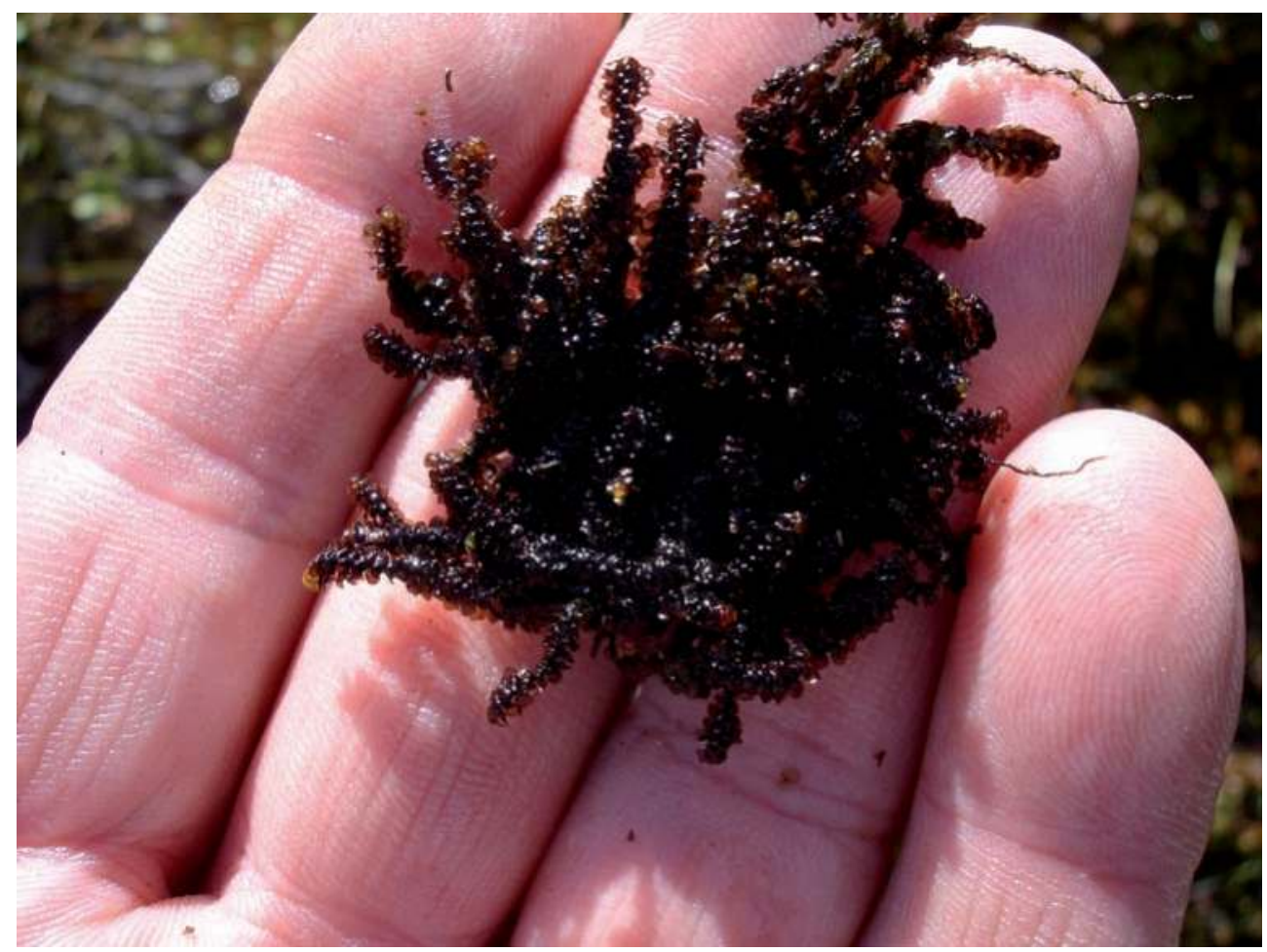

Abb. 12:

Gewelltes Spaten-Lebermoos (Scapania undulata) bildet oft großflächige schwärzlich-rote Bestände in Quellaustritten (Foto: Georg Gärtner)

lytrichum = Polytrichastrum sexangulare) sowie Vertreter der Gattungen Kiaeria und Pohlia wie z.B. das Gabelzahnmoos (Kiaeria falcata) und das Veränderliche Pohlmoos (Poblia drummondii). Typische Lebermoose dieses Spezialstandortes sind z.B. das Sudeten-Spitzmoos (Lophozia sudetica) und das Zweifelhafte Kopfsprossmoos (Cephalozia ambigua). Im Bereich von Schneetälchen finden sich weiters noch Moose, die auch auf Blockhalden verbreitet sind, wie z.B. das Zottige Haarmützenmoos (Racomitrium lanuginosum) und das Weißgabelzahnmoos (Paraleucobryum enerve). Auf offenen, durchfeuchteten Rohböden in Schneetälchen und Quellfluren bis in die alpine Stufe kommen weiters durch Wachsüberzug weißlichgraue, schimmelartige dichte Decken eines winzigen Lebermooses, Anthelia juratzkana, vor.

Viele der im 1. Band dieser Reihe (Koch \& Erschbamer 2010) im Anhang aufgelisteten Moose des Rotmoostales sind auch im übrigen Gebiet von Obergurgl in der subalpinen Stufe zu finden, eine 
neuerliche Auflistung kann daher unterbleiben. Ähnliches gilt für die Algenflora. Die Nomenklatur der Flechten richtet sich nach Hafellner \& Türk (2001), die der Moose nach Düll, R. (1991), Frey et al. (1995), Grims (1999), Hill et al. (2006) und Köckinger et al. (2008). Angaben zur Standortsphysiologie und Vergesellschaftung der Moose wurden, neben den bereits genannten Werken, aus Dierßen (2001) entnommen.

\section{Literatur}

Bortenschlager, S. (1984) Beiträge zur Vegetationsgeschichte Tirols I. Inneres Ötztal und Unteres Inntal. Ber. nat.-med. Ver. Innsbruck 71: 19-56.

Bortenschlager, S. (2000) Vegetationsgeschichte im Raum Obergurgl. In: Die subalpine und alpine Vegetation in $\mathrm{Ti}$ rol, Erschbamer, B., Grabner, S, (Hg.), Exkursionsführer: 27-31.

Bortenschlager, S. (2010) Vegetationsgeschichte im Bereich des Rotmoostales. In: Glaziale und Periglaziale Lebensräume im Raum Obergurgl, Koch, E. - M., Erschbamer, B. (Hg.), Alpine Forschungsstelle Obergurgl 1, innsbruck university press, Innsbruck: 77-91.

Dierßen, K. (2001) Distribution, ecological amplitude and phytosociological characterization of Europaean bryophytes.
Bryophytorum Bibliotheca 56, Cramer, Berlin, Stuttgart.

Düll, R. (1991) Die Moose Tirols. Unter besonderer Berücksichtigung des Pitztals/Ötztaler Alpen. IDH - Verlag, Bad Münstereifel, 442 S.

Erschbamer, B. (2000) Die alpine Stufe in den Zentralalpen. In: Die subalpine und alpine Vegetation in Tirol, Erschbamer, B., Grabner, S. (Hg.), Exkursionsführer: 114-146.

Frey, W., Frahm, J.-P., Fischer, E., Lobin, W. (1995) Kleine Kryptogamenflora. Begründet von H. Gams. Band IV. Die Moos- und Farnpflanzen von Europa. Gustav Fischer, Stuttgart, Jena, New York.

Gärtner, G. (2010) Zur Kryptogamenflora im Rotmoostal. In: Glaziale und Periglaziale Lebensräume im Raum Obergurgl, Koch, E.-M., Erschbamer, B. (Hg.). Alpine Forschungsstelle Obergurgl 1, innsbruck university press, Innsbruck: 145-154.

Grims, F. (1999) Die Laubmoose Österreichs, Biosystematics and Ecology 15, Catalogus Florae Austriae II, Bryophyten (Moose) 1, Musci (Laubmoose), Österr. Akad. Wiss., Wien, 418 S.

Hill, M. O., Bell, N., Bruggeman-Nannenga, M. A., Brugués, M., Cano, M. J., Enroth, J., Flatberg, K. I., Frahm, J.-P., Gallego, M. T., Garilleti, R., Guerra, J., Hedenäs, L., Holyoak, D. T., Hyvönen, J., Ignatov, M. S., Lara, F., Mazimpaka, V., Muñoz, J. \& Söderström, L . (2006) 
An annotated checklist of the mosses of Europe and Macaronesia. J. Bryol. 28: 198-267.

Hofmann, P., Türk, R. \& Gärtner, G. (1988)

Beitrag zur Flechtenflora Tirols: Obergurgl (Ötztaler Alpen, Nordtirol). Ber. nat.-med. Ver. Innsbruck 75: 7-19.

Koch, E.-M., Erschbamer, B. (2010) (Hg.) Glaziale und Periglaziale Lebensräume im Raum Obergurgl. Alpine Forschungsstelle Obergurgl 1, innsbruck university press, Innsbruck, $304 \mathrm{~S}$.

Köckinger, H., Suanjak, M., Schriebl, A. \& Schröck, C. (2008) Die Moose Kärntens. Sonderreihe Natur Kärnten 4, Naturwiss. Verein für Kärnten, Klagenfurt, 319 S.

Mitteilungen Forstliche Bundesversuchsanstalt (1959) Ökologische Untersuchungen in der subalpinen Stufe I: 1-430.

Mitteilungen Forstliche Bundesversuchsanstalt (1965) Beiträge zur subalpinen Waldforschung: 1-271.

Mitteilungen Forstliche Bundesversuchsanstalt (1967) Ökologie der alpinen Waldgrenze:1-492.

Nebel, M. (2001) Amblystegiaceae. Stumpfdeckelmoose. In: Die Moose BadenWürttembergs Band II, Nebel, M., Philippi, G. (Hg.), Eugen Ulmer, Stuttgart: 282-355.

Philippi, G. (2005) Scapaniaceae. Spatenmoose. In: Die Moose Baden-Württembergs Band III, Nebel, M., Philippi, G. (Hg.), Eugen Ulmer, Stuttgart: 350-377.

Pitschmann, H., Reisigl, H., Schiechtl, H. M., Stern, R. (1980) Karte der aktuel- len Vegetation von Tirol 1/100 000, VII Teil: Blatt 10, Ötztaler Alpen Meran. Doc. Cart. Ecol., 23: 47-68.

Sauer, M. (2001) Bartramiaceae. Apfelmoose. In: Die Moose Baden-Württembergs Band II, Nebel, M., Philippi, G. (Hg.), Eugen Ulmer, Stuttgart: 143-160.

Schiechtl, H. M. (1965) Die Vegetationskartierung im Rahmen der Wiederbewaldungsprobleme in der subalpinen Stufe (mit Vegetationskarte 1:37 500 des Gurgler Tales). Mitt. Forstl. Bundesvers. Anst. 59: 21-32.

Tranquillini, W. (1979) Physiological Ecology of the Alpine Timberline. Ecol. Stud. 31: 1-137.

Tranquillini, W. (2000) Alpine Waldgrenze. In: Die subalpine und alpine Vegetation in Tirol, Erschbamer, B., Grabner, S. (Hg.), Exkursionsführer: 42-45.

\section{Verzeichnis der Autoren}

Georg Gärtner

Institut für Botanik

Universität Innsbruck

Sternwartestr. 15, 6020 Innsbruck

Georg.Gaertner@uibk.ac.at

Wolfgang Hofbauer

Fraunhofer-Institut für Bauphysik

Holzkirchen, Fraunhoferstr. 10

83626 Valley

Wolfgang.Hofbauer@ibp.fraunhofer.de 


\title{
Kapitel 8 I Mineralogie und Petrologie des austroalpinen Kristallins in der südlichen Umgebung von Obergurgl -
}

\author{
Der Übergang vom Ötztal Komplex in den \\ Schneeberg Komplex (Ötztal, Österreich, \\ Passeiertal, Südtirol)
}

Peter Tropper, Kurt Krenn, Georg Hoinkes

\section{Zusammenfassung}

In den südlichen Ötztaler Alpen treten drei unterschiedliche tektonische Einheiten auf, nämlich der Ötztal Komplex (ÖC), der Schneeberg Komplex (SC) und der Texel Komplex (TC). Beide Einheiten, ÖC und SC, sind Teil des Oberostalpinen Deckensystems, wobei der ÖC das polymetamorphe Basement und der SC monometamorphe Reste einer paläozoischen, stark karbonatbetonten Sedimentbedeckung darstellen. In der Nähe der Grenze Österreich/Italien lässt sich die lithologische und tektonische Grenze zwischen diesen beiden Einheiten beobachten. Aufgrund des stark unterschiedlichen Gesteinsbestandes lassen sich die beiden Einheiten im Gaisbergtal relativ leicht voneinander unterscheiden. Der ÖC besteht hier im Wesentlichen aus feinkörnigen monotonen Paragneisen und Glimmerschiefern mit kleinen $(\leq 1 \mathrm{~mm})$ Granat- und Biotitkristallen, während der SC hauptsächlich aus grobkörni- gen $(0.5$ to $>1 \mathrm{~cm})$ Granatglimmerschiefern, Hornblendeschiefern und Marmoren besteht. Die Gesteine des SC sind Teil der sog. „Bunten Randserie“. Entlang der Timmelsjoch Passstraße ist die Unterscheidung nicht so einfach, da der äusserste Rand des SC und der des ÖC als monotone Paragneise vorliegen. Die Unterscheidung war daher nur mittels petrographischer und mineralchemischer Daten möglich. Im Gegensatz zum SC wurden die Gesteine des ÖC von der variszischen und eoAlpinen Metamorphose erfasst. Daher sind die Granate im ÖC chemisch diskontinuierlich zoniert. Im Zuge der eo-Alpinen Metamorphose kam es in den Gesteinen des ÖC zur Umwandlung der variszischen Staurolithe nach der Reaktion Staurolith + Albit $+\mathrm{H}_{2} \mathrm{O}$ $=$ Paragonit + Chlorit + Quarz. Dies führte zur Bildung von charakteristischen Glimmer + Chlorit + Quarz Pseudomorphosen nach Staurolith, die mehrere cm Länge erreichen und auf den Schieferungsflächen auftreten. 
Im Übergangsbereich ÖC/SC treten eo-Alpine Staurolithe auf, die durch den Einbau von $\mathrm{Zn}(\leq 5,6 \mathrm{Gew} \% \mathrm{ZnO})$ stabilisiert wurden. Die $P$-T Bedingungen für die eo-Alpine Metamorphose waren $550-600^{\circ} \mathrm{C}$ und $8-10$ kbar. Geodynamisch lässt sich die tektonisch hangende Position von ÖC auf SC und dem TC mit der Subduktion und Schließung des Meliata Ozeans während der eo-Alpinen Orogenese erklären.

\section{Abstract}

In the southern Ötztal Alps, three Austroalpine tectonic units occur from $\mathrm{N}$ to $S$ : the Ötztal Complex (ÖC), the Schneeberg Complex (SC) and the Texel Complex (TC). These units are part of the Upper Austroalpine basement nappes. The ÖC and the TC represent a polymetamorphic basement while the SC represents the remnant of a Paleozoic metasedimentary cover with normal stratigraphic relations and consists of several E-W trending synforms overturned to the $S$ with a strikingly different lithology compared to the adjacent ÖC. The rocks of the SC are eo-Alpine monometamorphic and sandwiched between the overlying ÖC and the underlying TC. Close to the Austrian/Italian border it is possible to trace the lithological and tectonic contact between the ÖC and SC with respect to their mineral assemblages and metamorphic overprint. The distinction between both units is unambiguous in the Gaisbergtal due to the difference in lithologies since the rocks of the ÖC are monotonous paragneisses and micaschists with abundant small $(\leq 1 \mathrm{~mm})$ garnet crystals and the rocks of the SC are mostly garnetmicaschists with large $(0.5$ to $>1 \mathrm{~cm})$ garnets, amphibole-bearing rocks with large $(\geq 1 \mathrm{~cm})$ amphiboles and marbles. Latter belong to the heterogeneous marginal series („Bunte Randserie") of the SC. Along the Timmelsjoch street the distinction is not so straight forward due to the occurrence of monotonous paragneisses in the outermost part of the SC as well as in the ÖC. The distinction was only possible using petrographic and mineral chemical characteristics. In contrast to the SC the ÖC gneisses were affected by both Variscan and eo-Alpine metamorphism, which led to discontinuous chemical garnet zoning. The subsequent eo-Alpine event in this area caused the breakdown of Variscan staurolite according to a reaction: staurolite + albite + $\mathrm{H}_{2} \mathrm{O}=$ paragonite + chlorite + quartz. This reaction caused the formation of characteristic mica + chlorite + quartz-pseudomorphs after staurolite that may reach several $\mathrm{cm}$ in length and can be found on cleavage planes. P-Tconditions sufficient for eo-Alpine staurolite formation, however, were reached at the transition from the ÖC to the SC and eo-Alpine staurolites that appear in micaschists of the ÖC and SC are rich in $\mathrm{Zn}$ with up to $5.6 \mathrm{wt} \%$ $\mathrm{ZnO}$. Equilibrium $P$-T-conditions for the eoAlpine event in the southern Ötztal Alps were $550-600^{\circ} \mathrm{C}$ and $8-10$ kbar. Further to the $S$ the rocks of the central SC main synform are encountered. That is characterized by rather monotonous garnet-micaschists (,Monotone Serie"). Geodynamically the position of the ÖC on top of SC and TC results from the 
subduction and closure of the Meliata Ocean during the eo-Alpine orogeny.

\section{Einleitung}

Die Alpen entstanden als bivergentes Faltengebirge infolge der Konvergenz der Europäischen und Afrikanischen (Apulische) Platten durch Überschiebungen einzelner Decken bzw. Deckensysteme im Zuge zweier Orogenesen (Gebirgsbildungen), beginnend im Jura vor ca. 180 Millionen (Ma) Jahren (Schmid et al. 2004). Diese Orogenesen sind das Resultat von großräumigen Plattenbewegungen an der Erdoberfläche und dokumentieren die Schließung mehrerer Ozeanbecken (Stampfli et al. 2001), wobei der Meliata Ozean in der Oberkreide (ca. $100 \mathrm{Ma}$ ) und die Penninischen Ozeanbecken im Tertiär (Eozän ca. $50 \mathrm{Ma}$ ) geschlossen wurden. Aufgrund der Schließung und Subduktion (= Abtauchen der ozeanischen Platte unter eine kontinentale Platte) von Ozeanbecken treten in den Alpen kontinentale und ozeanische Bereiche als Decken auf. Im Zuge der Orogenese werden die Gesteine von einer Metamorphose d.h. einer Umkristallisation unter hohen Drucken $(P)$ und Temperaturen (T) erfasst. Die Quantifizierung dieser Metamorphosebedingungen ( $P, T)$ bzw. der Anzahl dieser Wachstumsphasen, die in manchen Mineralen im chemischen
Zonarbau gespeichert ist, ist essentiell für die Rekonstruktion dieser geodynamischen Prozesse.

Seit den Arbeiten von Ampferer (1906) und Wegener (1912) war die Deckenlehre das wegweisende Konzept zur tektonischen Gliederung der Alpen. Mit der Einführung der Plattentektonik wurde die Deckenlehre als Bestandteil der Plattentektonik in einen umfassenderen Kontext gestellt. Den mächtigsten Anteil in den Ostalpen bilden die sog. Austroalpinen Decken. Dieses Austroalpin wird auch Ostalpin genannt und entspricht in etwa dem Ablagerungsraum des (heute nicht mehr vorhandenen) Meliata Ozeans respektive der Apulischen Platte nördlich des Periadriatischen Lineaments. Es handelt sich dabei um Basement („Grundgebirgseinheiten") - bzw. Sedimentdecken, die jetzt tektonisch in Form eines Deckenstapels vorliegen. Dieses Austroalpin wird heute von den meisten Autoren in ein Oberostalpin und ein Unterostalpin unterteilt (Schmid et al. 2004). Das Oberostalpin wurde von der eo-Alpinen Metamorphose mit sehr unterschiedlichen Metamorphosegraden erfasst. Die Unterostalpinen Decken repräsentieren den Teil Apuliens, der am nächsten am Penninischen Ozean positioniert war und daher einen passiven Kontinentalrand Apuliens darstellte.

Im Austroalpinen Basement westlich des penninischen Tauernfensters gibt es vier verschiedene Kristallinkomplexe, die dem Oberostalpin (Oberostalpines Basement) 


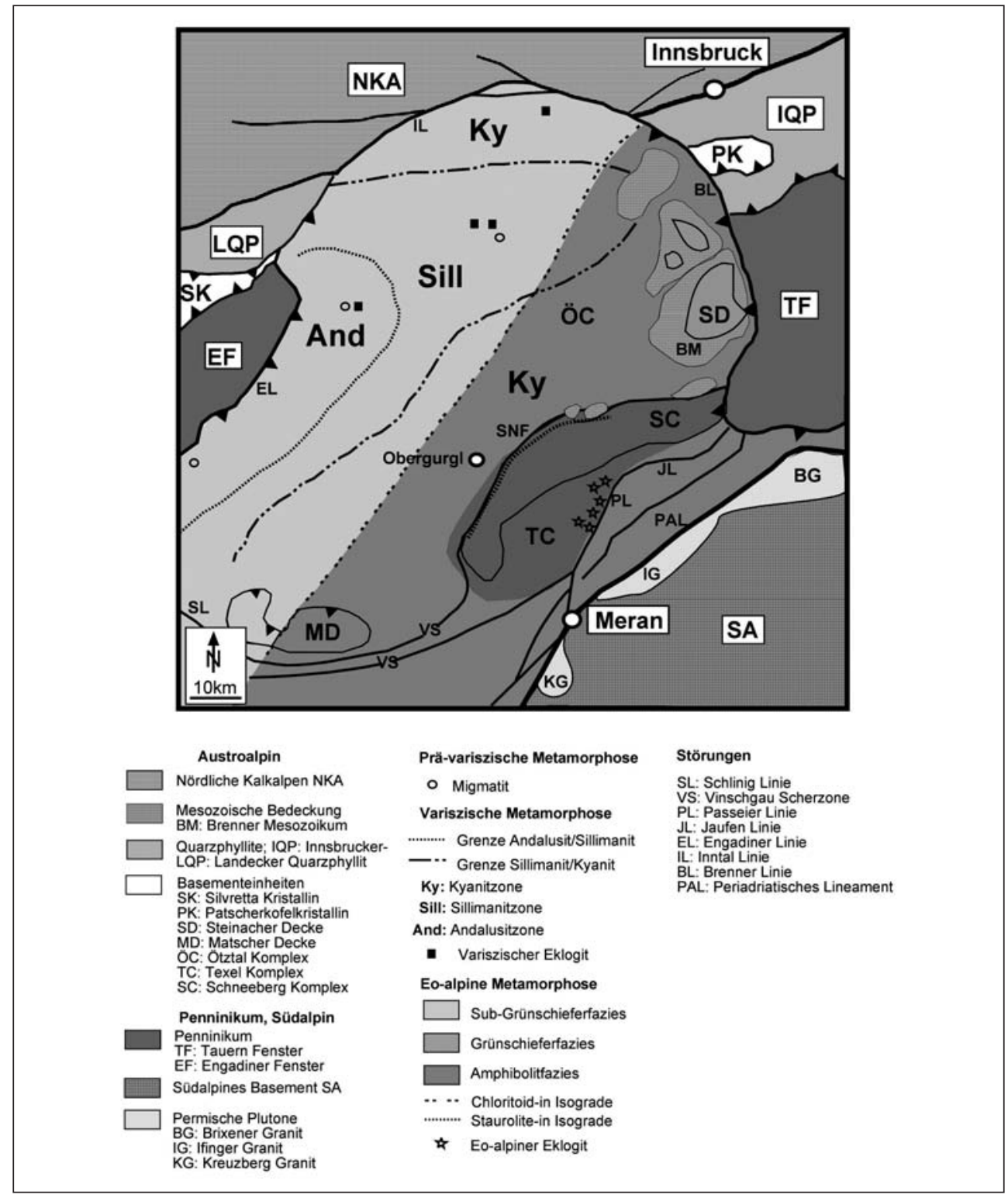

Abb. 1

Vereinfachter geologischer und petrologischer Überblick über die Kristallinkomplexe westlich des Tauern Fensters (modifiziert nach Tropper und Recheis, 2003). Die räumliche Verteilung der dominierenden Aluminiumsilikatphasen und die kretazische Chloritoid-In-Isograde (= räumlicher Bereich, ab dem das Indexmineral das erste Mal auftritt) wurden nach Purtscheller (1969) und die kretazische Staurolith-In-Isograde nach Hoinkes (1981) eingetragen. 
zugerechnet, aber aufgrund der unterschiedlichen Lithologien und Metamorphosegeschichten unterschieden werden können (Abb. 1). Es handelt sich nach Schmid et al. (2004) um 1) das ÖtztalBundschuh Deckensystem, zu dem der Ötztal Komplex (ÖC) gehört, 2) das Koralpe-Wölz Hochdruck Deckensystem, welches den Schneeberg (SC)- und den Texel (TC) Komplex beinhaltet, 3) das Silvretta-Seckau Deckensystem mit dem Campo-, Sesvenna- und Silvretta Kristallin und 4) das Drauzug-Gurktal Deckensystem, zu dem der Meran-Mauls Komplex gehört.

Lithologisch bestehen diese Kristallinkomplexe zum größten Teil aus klastischen Metasedimenten, wobei untergeordnet auch Orthogneise (ehemalige Granite), Amphibolite (ehemalige Basalte) und Metakarbonatgesteine auftreten können. Man kann außerdem vier verschiedene Typen von Gesteinen in diesen Kristallinarealen unterscheiden: 1) im Liegenden (= tiefsten Anteile) das sogenannte „Altkristallin“ (Grundgebirge, Basement), 2) in den hangenden (= höchsten) Anteilen bzw. innerhalb des Grundgebirges treten in manchen Bereichen retrograde Anteile dieses Basements, die durch Phyllonite (bei niedrigen Druck- und Temperaturbedingungen stark umgewandelte und deformierte Gesteine) repräsentiert werden, auf. Weiters liegen auf dem Grundgebirge 3) permo-mesozoische Metasedimente.
Untergeordnet treten 4) häufig magmatische Gänge auf, welche die Gesteine des Grundgebirges diskordant durchschlagen. Die prägende Orogenese und damit einhergehende Metamorphose in den südlichen Ötztaler Alpen ist die sog. eo-Alpidische Orogenese, bei der es zur Subduktion des Meliata Ozeans zwischen der Nordapulischen und der Südapulischen Platte vor ca. 100 Ma kam (Schmid et al. 2004). Der ÖC war Teil der Nordapulischen Oberplatte, während das Campo Kristallin Teil der Unterplatte war. Im Zuge dieser Subduktion kam es zur Bildung von Eklogiten, die heute im Texel Komplex, tektonisch zwischen dem ÖC und dem Campo Kristallin, zu finden sind (Sölva et al. 2005). Die anschließende Kollision führte zur WNW-gerichteten Deckenstapelung der Austroalpinen Decken. Die mit dieser Kollision assoziierte eo-Alpine Metamorphose erfasste alle Austroalpinen Decken mit unterschiedlicher Intensität. Im Tertiär kam es dann zur Subduktion der Alpinen Tethys, wobei die Austroalpinen Decken im Zuge dieser Alpidischen Orogenese auf die Penninischen Einheiten aufgeschoben wurden.

Kristallinkomplexe wie z.B. der ÖC spielen eine wichtige Rolle in der Untersuchung der prä-Alpinen Metamorphosegeschichte in den Ostalpen, da große Anteile fast keine oder nur eine schwache eoAlpidische Metamorphose erlebt haben. 


\section{Geologischer Überblick}

Im Bereich des hinteren Ötztales bei Obergurgl nahe des Grenzüberganges zwischen Österreich und Italien am Timmelsjoch und im nördlichsten Passeier Tal sind nach Sölva et al. (2005) folgende Kristallinkomplexe aufgeschlossen; der Ötztal Komplex (ÖC), der Schneeberg Komplex (SC) und der Texel Komplex (TC) (Abb. 1).

\subsection{Der Ötztal Komplex}

Lithologisch besteht der ÖC hauptsächlich aus polymetamorphen Basementgesteinen mit amphibolitfaziell (ca. 500- $6000^{\circ} \mathrm{C}$ und 4-8 kbar) metamorph geprägten mittel- bis feinklastischen $\mathrm{Me}$ tasedimenten, Orthogneisen und Amphiboliten. Im ÖC kann man drei weitere auflagernde Einheiten unterscheiden (Abb. 1):

1. Die Relikte einer Permo-Mesozoischen Sedimentbedeckung (Brenner Mesozoikum) findet man westlich der Brenner Störung und nördlich des Schneeberger Komplexes. Diese Bedeckung ist autochthon (= insitu).

2. Die Steinacher Decke und die Blaser Decke bestehen aus einem strukturell tieferen kristallinen Anteil von Gneisen und Amphiboliten an der Basis und einer metasedimentären, paläozoischen Bedeckung. Die beiden Decken wurden auf den ÖC mit seiner sedimentären Bedeckung aufgeschoben (= allochthon) und weisen daher eine kataklastische (= spröde) Störung an der Basis auf.

3. Die Matscher Decke im Süden bildet eine allochthone (überschobene) Decke aus Metasedimenten, die permische, magmatische Gänge aufweisen. Im Gegensatz zum ÖC wurde hier eine permische Metamorphose nachgewiesen (Habler et al. 2005). Diese Einheit wurde prä-eo-Alpin auf den ÖC geschoben.

Die Grenzen des ÖC sind rein tektonischer Natur (Abb. 1, Sölva et al. 2005). Der ÖC grenzt im Osten direkt an das Tauernfenster entlang der Brennerstörung (Brenner Linie). Bei der Brennerstörung handelt es sich um eine nach Westen einfallende Abschiebung mit einem Lateralversatz von 15 bis $26 \mathrm{~km}$ und ein Vertikalversatz von ca. $20 \mathrm{~km}$. Die tektonische Grenze im Norden zu den nördlichen Kalkalpen hin bildet die Inntalstörung (Inntal Linie). Es handelt sich um eine sinistrale (linksgerichtete) Seitenverschiebung mit einer südvergenten Überschiebungskomponente östlich des Kontakts mit der Brennerstörung. Im Süden ist die Brennerstörung eine fast vertikale dextrale Seitenverschiebung, die am Westrand des Tauernfensters entlangläuft und in das Periadriatische Lineament mündet. 
Im Südwesten bilden die Schlinig Linie und die Vinschgau Scherzone die Grenze. Entlang dieser tektonischen Grenze konnte ein Versatz nach Westen von ca. $45 \mathrm{~km}$ festgestellt werden. Die tektonische Abgrenzung im Südosten verläuft anschließend entlang des Schneeberger Komplexes (Sölva et al. 2005). Die tektonische Grenze im Westen bildet die Engadiner Störung (Engadiner Linie). Es handelt sich um eine NO-SW verlaufende Seitenverschiebung. Im Südosten des Engadiner Fensters handelt es sich um eine Abschiebung mit einem Vertikalversatz von ca. $3 \mathrm{~km}$.

\subsection{Der Schneeberg Komplex (SC)}

Der Schneeberg Komplex (SC) ist eine eigenständige tektonische Einheit und liegt im Südosten des ÖC (Abb. 2). Es handelt sich um mehrere enge, $\mathrm{O}-\mathrm{W}$ streichende Synformen (= Muldenstrukturen bestehend aus der Schneeberger Hauptmulde und den Seeberspitz-, Schrottner Synformen, Mauracher 1980). Der SC wird als paläozoische Bedeckung auf dem TC interpretiert (Hoinkes et al. 1987). Die Lithologien (= Gesteinsinhalt) des Schneeberg Komplexes sind im Gegensatz zum ÖC stark karbonatisch. Nach Mauracher (1980) wird die Schneeberger Hauptmulde lithologisch in die „Bunte Randse-

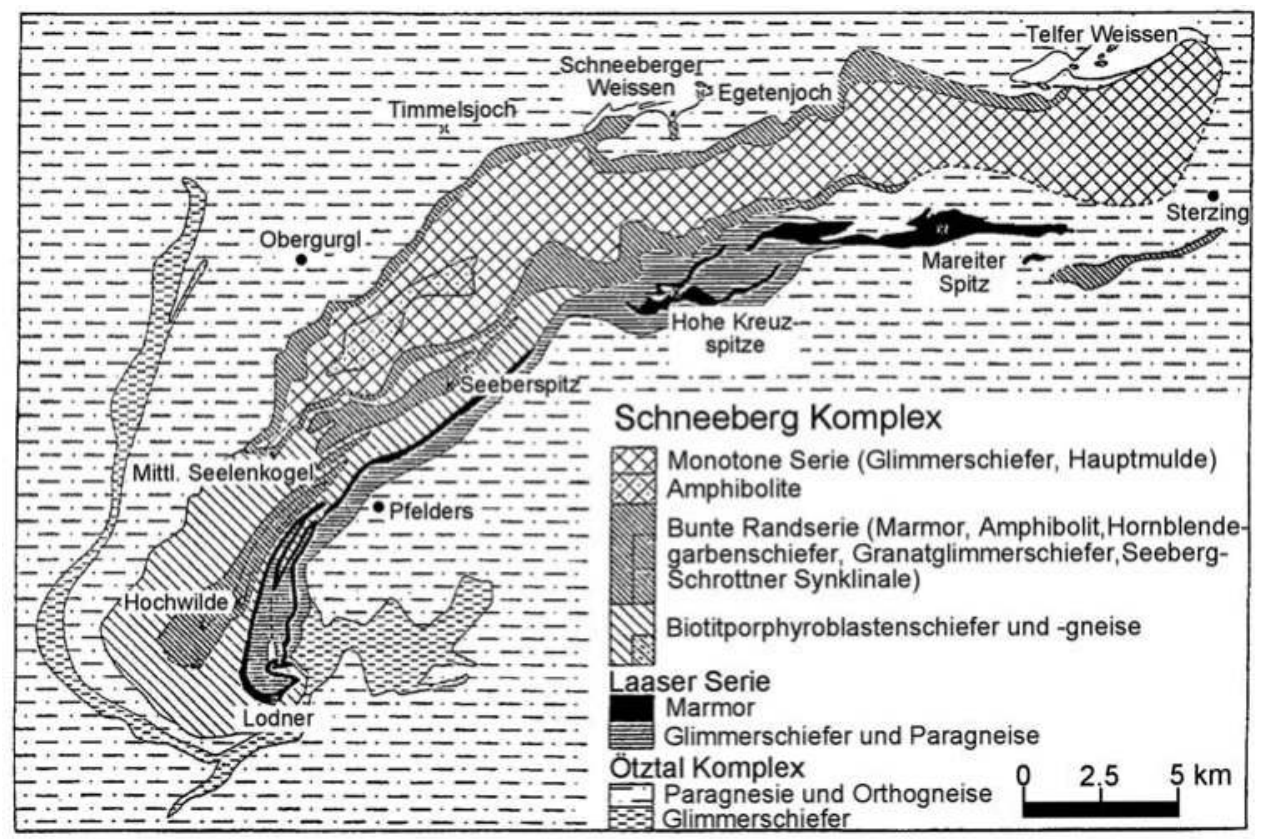

Abb. 2

Lithologische Gliederung des Schneeberg Komplexes (modifiziert nach Mauracher 1980) 
rie“ und die „Monotone Serie“ unterteilt. Die „Bunte Randserie“ tritt am Rand auf und besteht aus Wechsellagerungen von Granatglimmerschiefern, Amphiboliten, Karbonatschiefern, Quarziten und Marmoren. Die zentrale "Monotone Serie“ besteht aus einer Wechsellagerung von Granatglimmerschiefern und Quarziten.

\subsection{Der Texel Komplex (TC)}

Der Texel Komplex (TC) befindet sich südlich des SC (Abb. 1) und zeigt lithologisch starke Ähnlichkeiten zum ÖC. Im Gegensatz zum ÖC zeigt er aber eine eklogitfazielle eo-Alpine Überprägung (Habler et al. 2006). Eklogite treten im Bereich zwischen dem SC und der Passeier Störung auf (Hoinkes et al. 1991). Die Eklogite des TC stellen somit die westlichsten Vorkommen im Koralpe-Wölz Hochdruck Deckensystem dar (Sölva et al. 2005, Habler et al. 2006, Thöni 2006).

3. Die tekotonometamorphe Entwicklung von ÖC und SC

\subsection{Die Metamorphoseentwicklung}

Mindestens drei Metamorphoseereignisse lassen sich im ÖC unterscheiden, die jedoch regional unterschiedlich ausgeprägt sind (Abb. 1), nämlich die Kaledonische
Metamorphose (Ordovizium), die Variszische Metamorphose (Karbon) und die eo-Alpine Metamorphose (Oberkreide). Die Unterscheidung dieser Metamorphoseereignisse basiert hauptsächlich auf der Anzahl der im Gestein nachweisbaren Stadien des Mineralwachstums.

Die Kaledonische Metamorphose ist durch die lokale Bildung von Migmatiten (= Gestein, das eine partielle Aufschmelzung erlebt hat) charakterisiert. Diese treten z.B. am Reschenpass (Schweigl 1995), im Winnebachsee-Gebiet (Hoinkes et al. 1972, Hoinkes 1973, Klötzli-Chowanetz 2001, Thöny et al. 2008) und im Verpeiltal (Bernhard 1994) auf. Es gibt von diesen Migmatiten nur wenige $P-T$ Daten, die auf eine $T$-betonte Metamorphose von $670-750^{\circ} \mathrm{C}$ und Drucken von $<2.8$ kbar (Thöny et al. 2008) oder $>4$ kbar (Hoinkes 1973) hinweisen.

Während die Kaledonische Metamorphose nur sehr lokal begrenzt auftritt, ist die Variszische Metamorphose die dominante prä-Alpidische Metamorphose (Purtscheller et al. 1987, Hoinkes \& Thöni 1993). Die Metamorphosebedingungen werden mit $500-650^{\circ} \mathrm{C}$ und $4-8 \mathrm{kbar}$ angegeben (Hoinkes \& Thöni 1993, Tropper \& Hoinkes 1996, Tropper \& Recheis 2003). Die regionale Verbreitung der Indexminerale (= für bestimmte $P$ - und $T$ Bedingungen charakteristische Minerale) Kyanit, Sillimanit und Andalusit in Abb. 1 nach Purtscheller (1969) ist nach heutigem Wissenstand nicht das Resultat eines einzigen Metamorphoseereignisses. Von 
besonderer Bedeutung ist das Auftreten von Eklogiten (= Hochdruckgestein mit basaltischem Ausgangschemismus) im zentralen ÖC, welche eine frühvariszische Hochdruckmetamorphose anzeigen. Die eklogitfaziellen Bedingungen werden mit $<730^{\circ} \mathrm{C}$ und $<27$ kbar angegeben (Miller \& Thöni 1995).

Die eo-Alpine Metamorphose weist im ÖC eine Metamorphosezonierung mit ansteigendem Metamorphosegrad von NW nach SO auf wie in Abb. 1 dargestellt (Thöni 1981, Hoinkes et al. 1982, Purtscheller et al. 1987, Thöni \& Hoinkes 1987, Hoinkes et al. 1999). Im NW wurde maximal die untere Grünschieferfazies, im SO wurden amphibolitfazielle Bedingungen erreicht (Hoinkes et al. 1991). Die T-Zunahme von $<300^{\circ} \mathrm{C}$ auf $550-600^{\circ} \mathrm{C}$ äußert sich durch das Auftreten der Indexminerale Stilpnomelan, Chloritoid, Granat und Staurolith in den Metapeliten (Purtscheller 1969, Hoinkes et al. 1982). Diese Metamorphosezonierung ist auch an post-variszischen Diabasgängen, die im gesamten ÖC verbreitet auftreten und in den PermoMesozoischen Einheiten ersichtlich sind (Purtscheller \& Rammlmair 1982). Der Höhepunkt der eo-Alpinen Metamorphose westlich des Tauernfensters wird im TC erreicht (Hoinkes et al. 1991). Es kommt zur Bildung von Eklogiten. Die $P-T$ Bedingungen werden von Hoinkes et al. (1991) mit 11-12 kbar für den Druckhöhepunkt und $600-650^{\circ} \mathrm{C}$ für den Temperaturhöhepunkt angegeben.
Habler et al. (2006) ermittelten leicht erhöhte $P-T$ Bedingungen von $540-620^{\circ} \mathrm{C}$ und 12-14 kbar.

Im Gegensatz zum polymetamorphen ÖC und TC zeigt sich im SC nur die eoAlpine Metamorphose (Hoinkes 1981, 1983, 1986a, b). Die Granate sind dementsprechend chemisch einphasig zoniert, da sie nur während eines einzigen Metamorphoseereignisses gewachsen sind. Die kretazische Metamorphose erreicht im Schneeberger Komplex 550-600 ${ }^{\circ} \mathrm{C}$ und 8-10 kbar (Hoinkes 1981, 1986a, b; Konzett \& Hoinkes 1996, Tropper \& Recheis 2003, Krenn 2010).

\subsection{Geochronologie}

Die Korrelation der im Gestein nachweisbaren Stadien des Mineralwachstums mit den ermittelten Altersdaten (= geochronologische Daten) erlaubt die exakte Zuordung einer Mineralparagenese (=Mineralgesellschaft in einem Gestein) zu einem Metamorphoseereignis und daher zu einer Orogenese. In den untersuchten Einheiten lassen sich die Alter in vier Gruppen unterteilen:

Prä-Kaledonische Alter: Dieses Alter wurde aus Metagabbros im zentralen ÖC ermittelt und ergaben 530-520 Ma (Miller \& Thöni 1995). Ähnliches Alter wurde auch an einem Tonalitstock (Klopaier Tonalit) gemessen (Schweigl 1995). Söllner (2001) und Söllner und Hansen (1987) ermittelten ein pan-Afrikanisches Alter 
von 670-607 Ma aus den Gesteinen des Winnebach Migmatits.

Die Kaledonische Metamorphose: Sie wurde nur im ÖC nachgewiesen. Das Alter der Migmatitbildung wurde am Winnebachmigmatit ermittelt und ergab mithilfe der U-Pb Einzelzirkondatierung ein Alter von $490 \pm 9 \mathrm{Ma}, 444 \pm 4$ und $455 \pm 4$ Ma (Klötzli-Chowanetz et al. 1997). Für die Migmatite am Reschenpass wurde ein Mindestalter von $470 \mathrm{Ma}$ aufgrund der Datierung von Pegmatitgängen abgeleitet (Schweigl 1995). Thöny et al. (2008) ermittelten mittels U-Th-Pb Datierung von Monaziten mit der Elektronenstrahl-

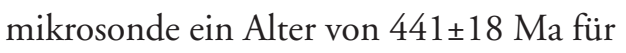
drei Migmatitvorkommen (Winnebach-, Reschenpass-, Verpeilmigmatit). Das Alter der Orthogneise im ÖC reicht von 470-430 Ma und wird als Intrusionsalter interpretiert (Hoinkes et al. 1997).

Die Variszische Metamorphose: Das frühvariszische Alter der eklogitfaziellen Metamorphose im zentralen ÖC wurde mithilfe der Sm-Nd Datierung von Granat und Klinopyroxen mit 350-360 Ma bestimmt (Miller \& Thöni 1995). Der T-Höhepunkt der nachfolgenden amphibolitfaziellen Metamorphose wurde um 343-331 Ma erreicht (Hoinkes et al. 1997).

Die eo-Alpine Metamorphose: Sie zeigt eine Metamorphosezonierung mit ansteigendem Metamorphosegrad von NW nach SO an (Abb. 1). Diese Zonierung wird auch durch die systematische Variation der Rb-Sr und K-Ar Alter von Muskoviten und Biotiten bestätigt, welche einen deutlichen Verjüngungstrend von NW nach SO zeigen (Thöni 1981). Während im NW variszische Abkühlalter vorherrschen, kommt es im zentralen Bereich des ÖC zur Bildung einer Mischalterzone und im SO zu einer Zone eo-Alpiner Abkühlalter. Das Alter des thermischen Höhepunktes der eo-Alpinen Metamorphose in den austroalpinen Einheiten wird mit ca. 100 $\pm 10 \mathrm{Ma}$ angegeben (Thöni 1999). Das Alter des Druckmaximums der eoAlpinen Metamorphose im TC wurde ursprünglich von Hoinkes et al. (1991) mittles Phengitdatierungen mit $143 \mathrm{Ma}$ angegeben. Granatdatierungen von Habler et al. (2006) ergaben aber ein jüngeres eo-Alpines Metamorphosealter von $85 \pm 5$ Ma. Im Gegensatz zum ÖC findet man im TC nur eo-Alpine Alter.

Geochronologische Untersuchungen im SC ergaben ein eo-Alpines Alter der Metamorphose und Abkühlung von 100-75 Ma (Thöni 1999).

\subsection{Die strukturelle Entwicklung}

Die austroalpinen Einheiten wurden während der vor-Alpinen und eo-Alpinen Orogenesen stark intern deformiert, wobei im Gelände planare Gefüge (= Schieferungen) und Faltenstrukturen erkennbar sind. Der ÖC zeigt auch regional unterschiedliche strukturelle Entwicklungen. Im nördlichen Anteil gibt es O-W streichende Faltenachsen, während im südlichen Anteil steil stehende Faltenach- 
sen („Schlingentektonik“ nach Schmidegg 1936, 1964) auftreten. Es lassen sich im Süden im Bereich der Weißkugel mindestens vier Deformationsphasen $\mathrm{D}_{1}-\mathrm{D}_{4}$ im ÖC nach Van Gool et al. (1987) unterscheiden. Die $\mathrm{D}_{1}-\mathrm{D}_{3}$ Strukturen wurden zum Variszischen Zyklus und $\mathrm{D}_{4}$ der Alpidischen Deformation zugerechnet. Bei den variszischen Deformationen handelt es sich um duktile (plastisch verformte) Strukturen, die auch Faltungen (z.B. Isoklinalfalten) beinhalten. $\mathrm{D}_{4}$ ist durch die Bildung von west-gerichteten Scherzonen im duktil/spröd (= bruchhaft) Übergangsbereich in der späten Kreide, charakterisiert.

Nach Fügenschuh (1995) lassen sich am Ostrand des ÖC mindestens drei duktile alpidische Deformationsphasen $\left(\mathrm{D}_{1}\right.$ $\mathrm{D}_{3}$ ) nachweisen. $\mathrm{D}_{1}$ findet in der späten Kreide und frühen Tertiär statt, während die letzten zwei Phasen beide im Tertiär stattfanden. Es lassen sich auch prä- $\mathrm{D}_{1}$ Strukturen ermitteln, die mit dem letzten Ereignis $\left(\mathrm{D}_{4}\right)$ von Van Gool et al. (1987) korrelierbar sind.

Im SC lassen sich fünf Deformationsphasen $\mathrm{D}_{1}-\mathrm{D}_{5}$ unterscheiden, wobei $\mathrm{D}_{1}-\mathrm{D}_{4}$ duktil sind und $\mathrm{D}_{5}$ spröd ist (Sölva et al. 2005). Die $D_{1}$ Deformation lässt sich mit dem Druckhöhepunkt vor ca. 98-86 Ma korrelieren. Die amphibolitfazielle Metamorphose überdauerte $\mathrm{D}_{1}$ und statisches Mineralwachstum überprägte $\mathrm{D}_{1}$ Mikrostrukturen (Habler et al. 2001, Sölva et al. 2005, Krenn 2010).
Der TC zeigt ebenfalls eine komplexe strukturelle Entwicklung mit vier Deformationsstadien $\mathrm{D}_{1}-\mathrm{D}_{4}$, wobei alle Stadien duktile Deformation aufweisen. $\mathrm{D}_{1}$ fand während der eklogitfaziellen Metamorphose statt, alle anderen Phasen gehören dem retrograden $P-T$ Pfad der Gesteine an (Sölva et al. 2005).

\section{Lithologisches Profil durch den Kontakt ÖC-SC}

Im Bereich des hinteren Ötztales ist der Kontakt zwischen dem ÖC und dem SC an mehreren Stellen sehr gut aufgeschlossen. Detaillierte Daten zum Kontakt zwischen beiden Einheiten liegen aus dem Gaisbergtal bei Obergurgl und entlang der Timmelsjoch Paßstrasse (Pedevilla 2011) nahe der Grenze Österreich/Italien auf Südtiroler Seite vor. Die beiden Einheiten ÖC und SC fallen alle nach NW ein, auffallend ist aber der starke lithologische Wechsel zwischen den beiden Einheiten. Während der ÖC hauptsächlich aus monotonen, feinkörnigen Glimmerschiefern und Paragneisen mit Einschaltungen von Quarziten und untergeordnet Amphiboliten besteht, treten im SC im Bereich der „Bunten Randserie“ eine Vielzahl von Lithologien auf, wie im Profil im Gaisbergtal in Abb. 3 dargestellt. Am Kontakt entlang der Timmelsjoch Paßstrasse wurde auf die Gesteine der „Bunten Randse- 


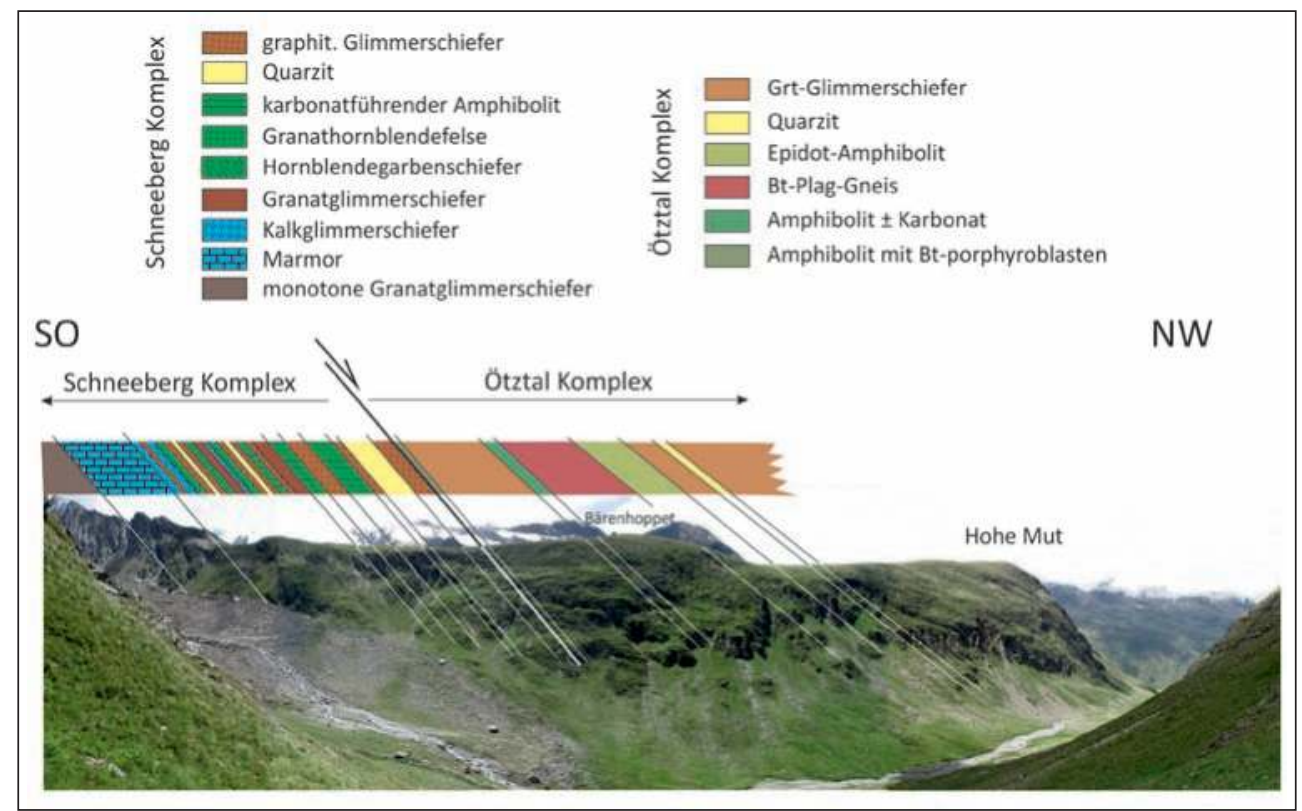

Abb. 3

Lithologisches Profil des Kontaktes zwischen dem ÖC und dem SC im Gaisbergtal nahe Obergurgl

rie“ eine ca. 100 m mächtige Abfolge von monotonen Paragneisen mit ausgeprägter Biotitblastese (Biotitwachstum) beobachtet (Abb. 4). Dieser Gesteinstyp wurde schon von Schmidegg (1932) als „Grenzglimmerschiefer bzw. Biotitporphyroblastenschiefer" auskartiert. Daran schließen die Gesteine des ÖC an. Da es sich um feinkörnige Paragneise handelt, lässt sich die Grenze zwischen beiden Einheiten nur mittels zusätzlicher petrographischer und mineralchemischer Untersuchungen feststellen. Die charakteristischen Lithologien in der „Bunten Randserie“ des SC sind Hornblende-Garbenschiefer, Kalksilikatschiefer, Marmorlagen und grobkörniger Granatglimmerschiefer. Die Mäch- tigkeit dieser Lithologien variiert von 0.5 bis $10 \mathrm{er}$ Meter.

Abb. 5 zeigt den Übergang zwischen der „Bunten Randserie“ und den monotonen Paragneisen mit der Biotitblastese (= Biotitwachstum) entlang der Timmelsjochstraße. Hier lässt sich der Kontaktbereich zwischen den monotonen Gneisen des äußersten Randes des SC und den Amphibol-dominierten Lithologien der „Bunten Randserie“ festlegen. Abb. 6 zeigt dann den tatsächlichen Kontakt (rote Linie) zwischen dem SC (links unterhalb) und dem ÖC (rechts oberhalb). $\mathrm{Da}$ es sich bei den Gesteinen beider Einheiten aber um monotone Paragneise 


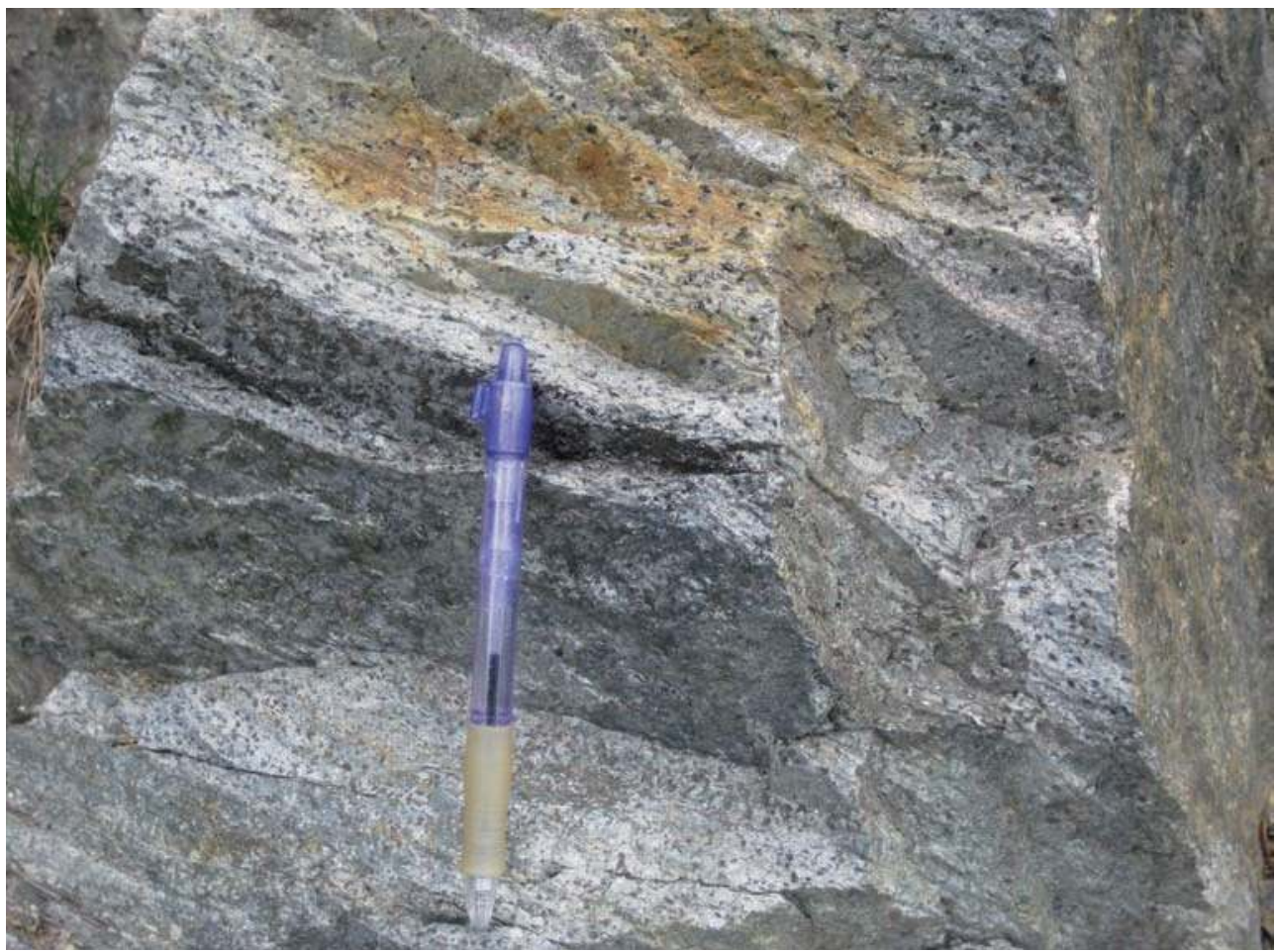

Abb. 4

Foto eines monotonen Paragneises aus dem Randbereich des SC mit deutlicher Biotitblastese (= Biotitwachstum, Pedevilla 2011)

handelt, war eine Unterscheidung beider Einheiten nur mittels petrographischer und mineralchemischer Methoden möglich (s. Diskussion).

\section{Petrographie}

Abb. 7 zeigt Durchlichtaufnahmen am Polarisationsmikroskop von ausgewählten mineralreichen Lithologien des ÖC und SC aus dem Umgebungsbereich von Obergurgl.

\section{1. Ötztal Kristallin}

Im Gaisbergtal treten im ÖC vereinzelt feinkörnige Amphibolite auf. Im direkten Kontakt zum SC weist diese Lithologie Biotitporphyroblasten auf (Abb. $7 A)$. Nördlich des Kontaktes tritt im ÖC ein markanter Epidot-Amphibolit auf (Abb. 7B). Charakteristisch für das süd- 


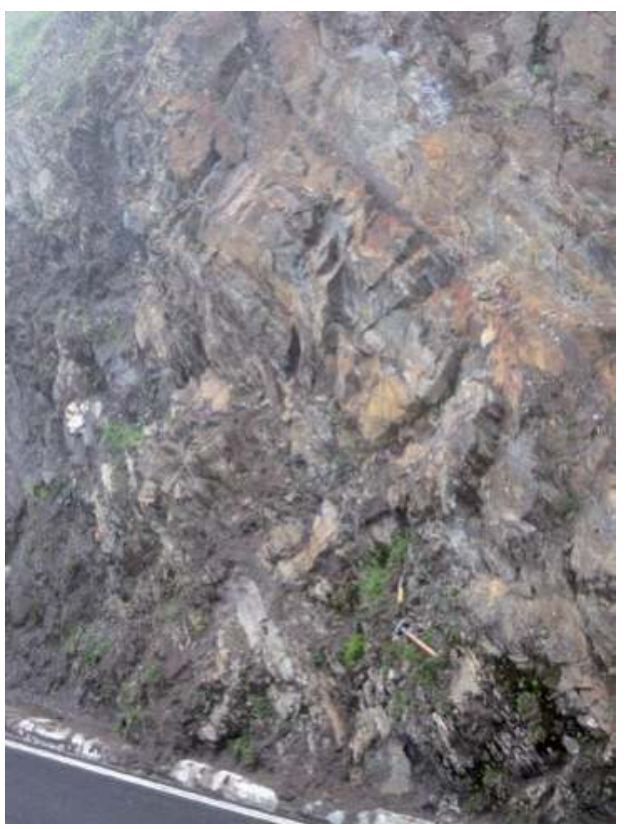

liche ÖC sind Granatglimmerschiefer mit Hellglimmerpseudomorphosen nach Staurolith (Abb. 7C). Nahe der Zollhütte am Timmelsjoch erreichen diese Pseudomorphosen (= komplettes Ersetzen des Wirtsminerals durch später gewachsene Minerale) mehrere cm Durchmesser. Variszische Staurolithe wurden durch Granat, Chlorit und Paragonit ersetzt, da die P-T Bedingungen zur Zn-freien Staurolithbildung während der eo-Alpinen Metamorphose in diesem Bereich noch nicht erreicht wurden (Hoinkes 1981). Daher zerfällt Staurolith nach folgender Mineralreaktion (Hoinkes 1981):

Staurolith + Albit $+\mathrm{H}_{2} \mathrm{O}=$ Paragonit + Chlorit + Quarz
Abb. 5:

Übergang zwischen der „Bunten Serie“ und den monotonen Paragneisen mit Biotitblastese am Rand des SC entlang der Timmelsjochstraße. Der Hammer liegt im Kontaktbereich. Rechts darüber (= hangend) treten die feinkörnigen, monotonen Paragneise mit ausgeprägtem Biotitwachstum auf. Links darunter (= liegend) befinden sich gröberkörnigere Granatglimmerschiefer, Amphibolite und eine Marmorlinse, die der „Bunten Randserie“ angehören.

Granate in dieser Probe sind feinkörnig, weisen jedoch einschlussreiche Kerne auf. Eine mikrostrukturelle Abtrennung voralpidischer und alpidischer Granatgenerationen ist schwierig und nur anhand der Mineralzusammensetzung eindeutig durchführbar (siehe Diskussion).

\subsection{Schneeberg Komplex}

Der SC führt im Unterschied zum ÖC Marmorlagen, die ausschließlich in der „Bunten Randserie“ auftreten. Die Marmore enthalten farblosen Amphibol, nämlich Tremolit (Abb. 7D). Häufig sind Kalksilikatgesteine wie Kalkglimmerschiefer zu beobachten (Abb. 7E, F). Diese enthalten Karbonate (Kalzit, Dolomit) 


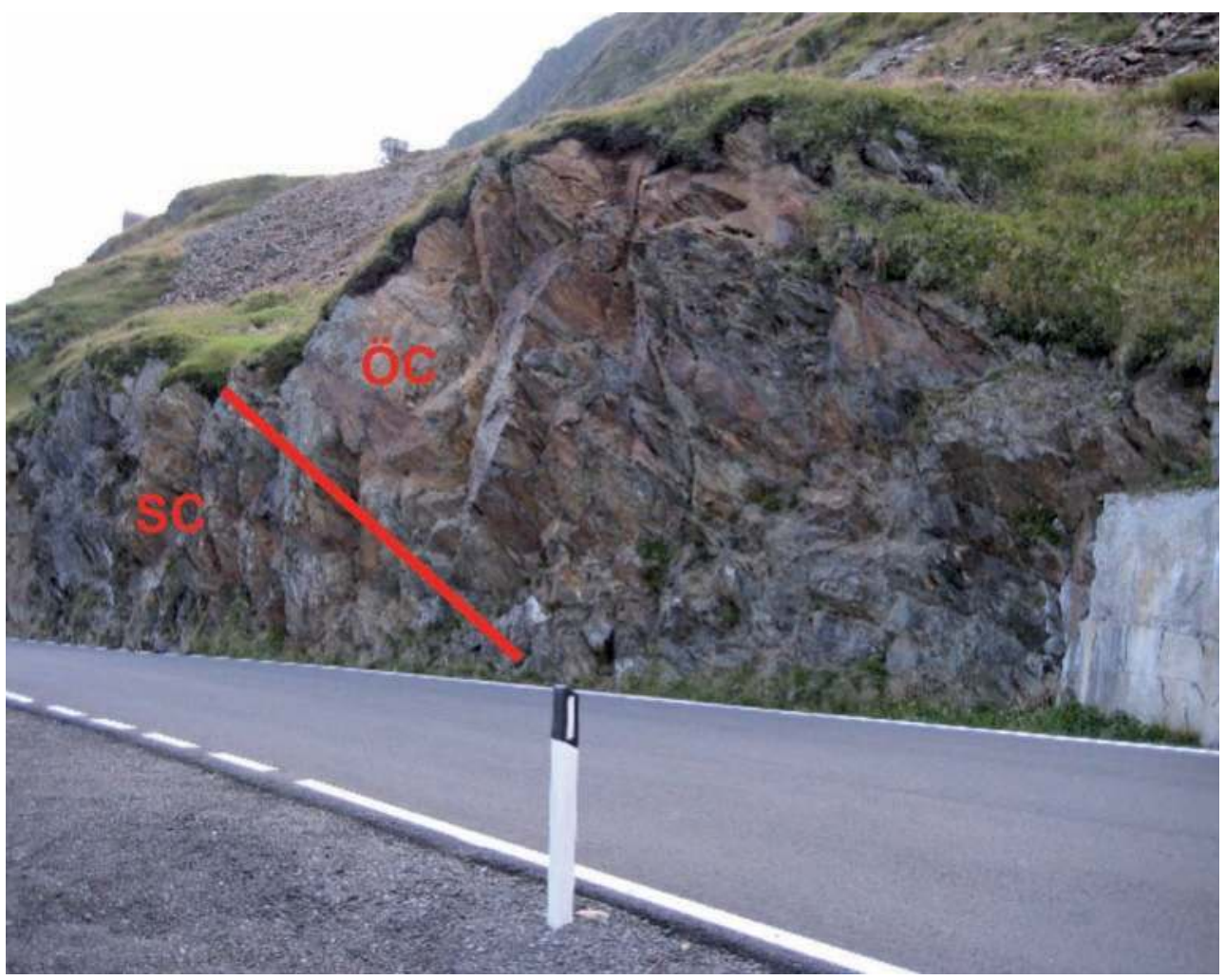

Abb. 6

Kontakt zwischen den monotonen Paragneisen mit Biotitblastese aus dem SC und den Paragneisen des ÖC. Wegen der Ähnlichkeit der Gesteine in beiden Einheiten konnte der Kontakt nur mittels petrographischer und mineralchemischer Untersuchungen festgelegt werden (Pedevilla 2011).

neben Silikaten (Klinozoisit, Muskovit, Biotit, Quarz). Die Glimmerschiefer des SC enthalten häufig Graphit und führen manchmal Kyanit (Abb. 7G). Charakteristisch für den SC sind paragonitführende Hornblende-Schiefer und -Felse (Abb. 7I), die manchmal Kyanit aufweisen (Abb. $7 \mathrm{H})$. Paragonit wird randlich oft retrograd durch die Paragenesen Plagioklas + Chlorit + Margarit, oder Plagioklas + Biotit + Margarit + Chlorit ersetzt. Die
Koexistenz von Paragonit mit Ca-Amphibol ist signifikant für eine druckbetonte amphibolitfazielle Metamorphose mit geringen geothermischen Gradienten im P-T Bereich der Epidot-Amphibolitfazies (Konzett \& Hoinkes 1996). Die Gesteine der „Monotonen Serie“ sind überwiegend Granatglimmerschiefer, in denen bereits Zn-reicher Staurolith wächst (Abb. 7J). Hoinkes (1981) beschreibt in den Proben aus dem nördlichsten SC ein beginnendes 

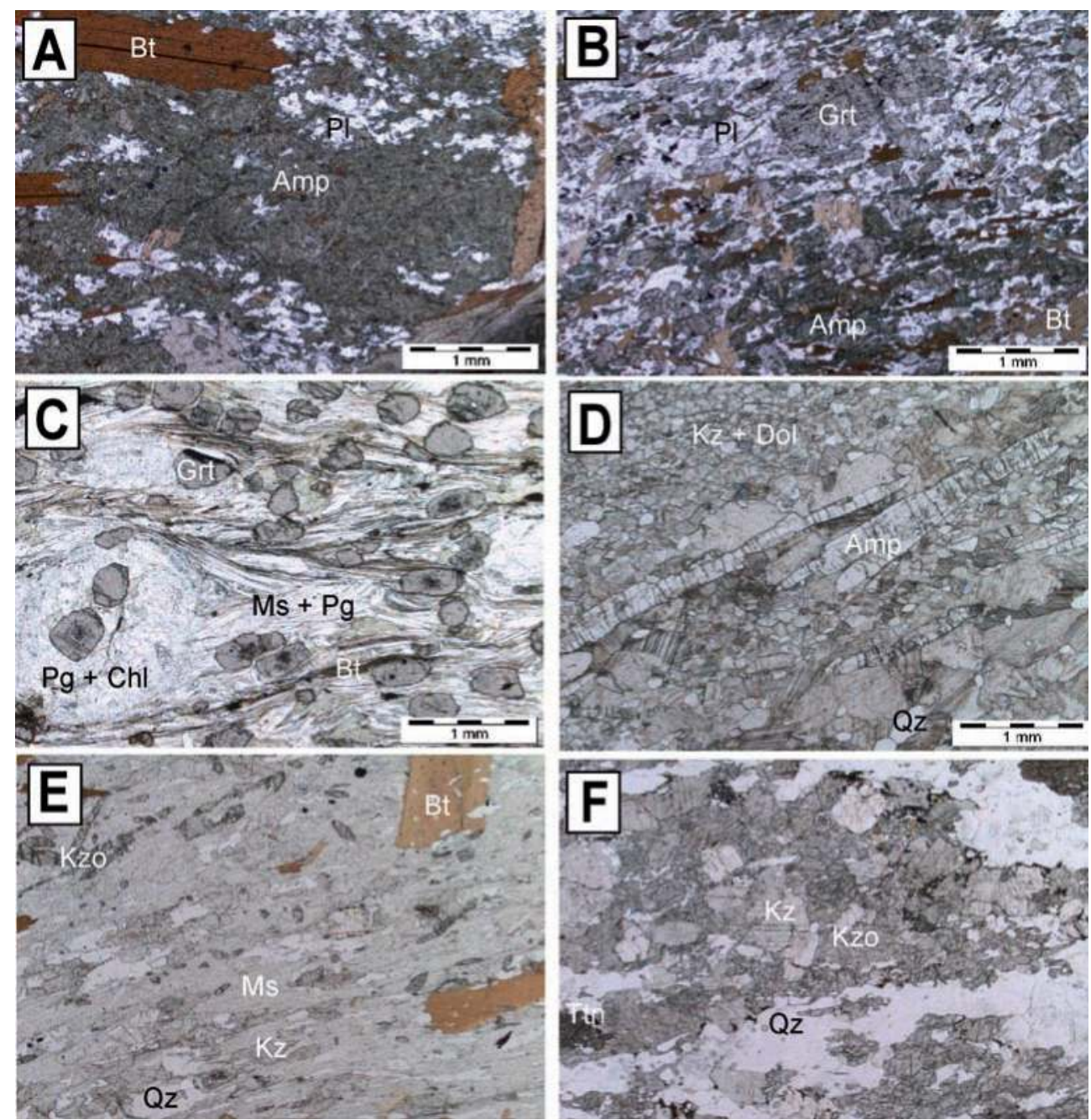

Abb. 7

Dünnschlifffotografien ausgewählter Lithologien aus dem ÖC (A-C) und dem SC (D-J)

A: Feinkörniger Amphibolit aus dem direkten Kontaktbereich ÖC/SC (Gaisbergtal)

B: Epidot-führender Amphibolit, im Liegenden des Kontakts (Gaisbergtal)

C: Granatglimmerschiefer mit Paragonit-Chloritpseudomorphosen nach Staurolith (Königstal)

D: Tremolitführender Marmor (Rotmoostal)

E: Kalkglimmerschiefer (Gaisbergtal)

F: Kalksilikatschiefer (Gaisbergtal) 

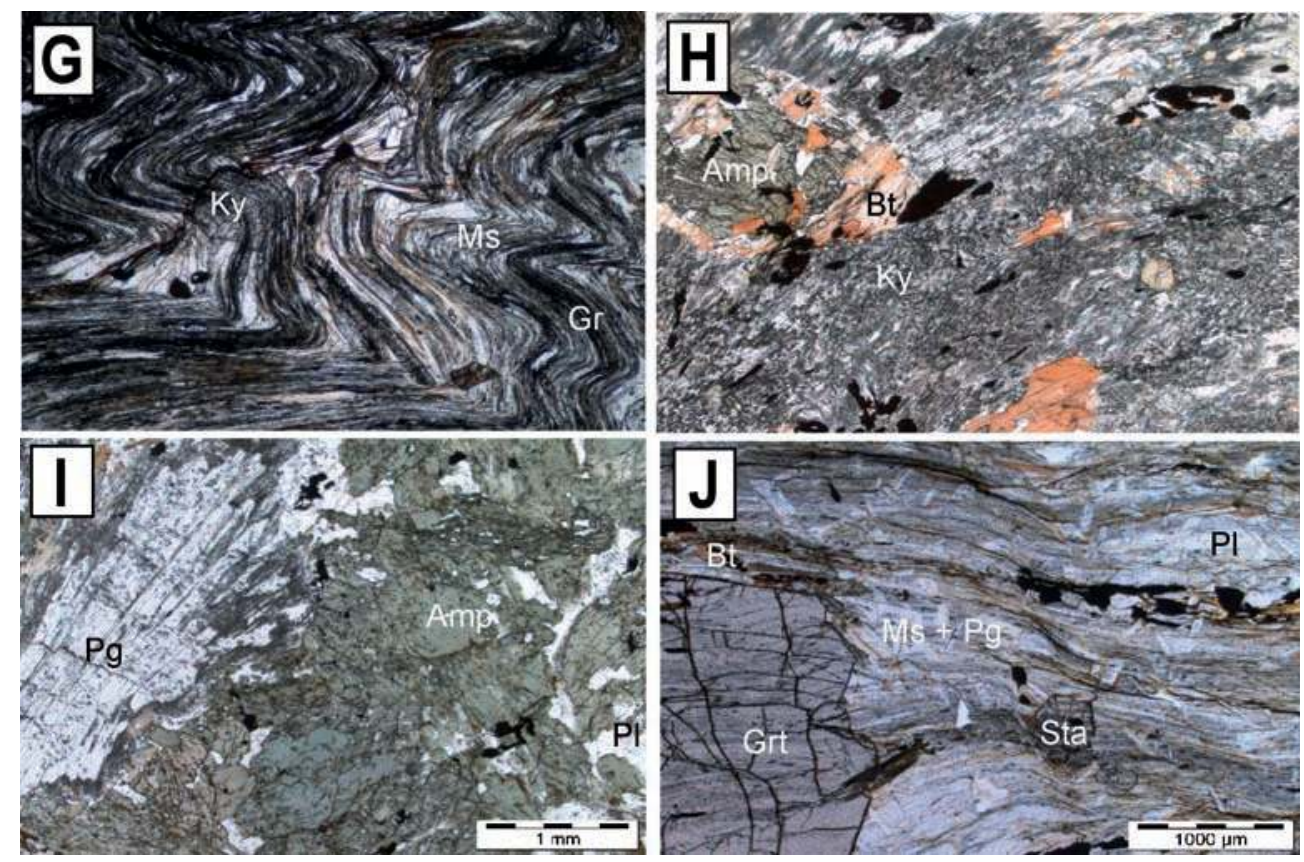

Abb. 7 - Fortsetzung

Dünnschlifffotografien ausgewählter Lithologien aus dem ÖC (A-C) und dem SC (D-J)

G: Kyanit-führender Graphitschiefer (Seewertal)

$\mathrm{H}$ : Kyanit-führender Hornblendeschiefer (Seewertal)

I: Paragonit-führender Amphibolit (Seewertal)

J: Staurolith-führender Granatglimmerschiefer aus der „Monotonen Serie“ des SC (Rotmoostal).

Abkürzungen:

Amp: Amphibol, Ky: Kyanit, Ms: Muskovit, Bt: Biotit, Grt: Granat, Pl: Plagioklas, Pg: Paragonit, Kz: Kalzit, Kzo: Klinozoisit, Gr: Graphit, Chl: Chlorit, Sta: Staurolith, Qz: Quarz.

Wachstum von postdeformativem Staurolith und Kyanit in graphitfreien als auch graphitführenden Glimmerschiefern. Selten ist ein Auftreten von mm-großen Staurolithen (Hoinkes 1981). Paragonit tritt als dominante Hellglimmervarietät neben Muskovit auf, sodass Kyanit und Staurolith u.a. nach folgenden Reaktionen gebildet wurden (Hoinkes 1981,
Proyer 1989):

Paragonit + Quarz =

Kyanit + Albit $+\mathrm{H}_{2} \mathrm{O}$

Paragonit + Granat + Quarz $=$

Staurolith + Plagioklas $+\mathrm{H}_{2} \mathrm{O}$

Paragonit + Biotit + Granat + Quarz $=$

Staurolith + Plagioklas + Muskovit $+\mathrm{H}_{2} \mathrm{O}$ 


\section{Mineralchemie}

Die mineralchemischen Untersuchungen aus dem Übergangsbereich ÖC/SC basieren auf den Arbeiten von Hoinkes (1981), Konzett \& Hoinkes (1996) sowie Habler et al. (2001) und Tropper \& Recheis (2003). Es werden hier nur die wichtigsten, indikativen Minerale besprochen. Es handelt sich bei diesen Mineralen um Silikate, wobei Granat und Staurolith der Gruppe der Inselsilikate, Hellglimmer der Gruppe der Schichtsilikate und Amphibol der Gruppe der Bandsilikate zugeordnet werden.

\subsection{Granat}

Hoinkes (1981) sowie Tropper \& Recheis (2003) beschreiben eine deutliche Änderung im chemischen Zonarbau zwischen dem südlichen ÖC und dem angrenzenden SC. Während im SC kontinuierlich zonierte chemisch einphasige Granate (= eine Wachstumsphase, d.h. ein Metamorphoseereignis) auftreten, findet man im ÖC diskontinuierlich zonierte chemisch zweiphasige Granate (= zwei Wachstumsphasen, d.h. zwei Metamorphoseereignisse). Eine erste Darstellung chemisch zonierter Granate kann mittels rückgestreuter Elektronenbilder (Back Scattered Electron Images: BSE) gemacht werden, da die Anzahl der rückgestreuten Elektronen aus der Probe mit der Höhe der mittleren Ordnungszahl der chemischen Elemente, die das Mineral aufbauen, zunimmt. D.h. Minerale mit schwereren mittleren Ordnungszahlen erscheinen heller im Bild. Man sieht daher bereits in Abb. 8A nur im Mn-Gehalt (= Spessartinkomponente $\left[\mathrm{Mn}_{3} \mathrm{Al}_{2} \mathrm{Si}_{3} \mathrm{O}_{12}\right]$ ) eine räumliche Zonierung, wobei es im Kern angereichert ist. Hingegen sind in den Granaten der Probe 9A bereits chemisch zwei unterschiedliche Granatgenerationen zu erkennen, da die untersuchten Elemente eine sprunghafte Änderung in ihrer Konzentration aufweisen. Da bei der Elektronenstrahlmikrosondenanalyse für jedes Element charakteristische Röntgenstrahlen aus der untersuchten Probe emittiert werden, kann die räumliche Verteilung der Intensitäten dieser Röntgenstrahlen verwendet werden, um Chemismusunterschiede der Elemente $\mathrm{Mg}$ (= Pyropkomponente $\left.\left[\mathrm{Mg}_{3} \mathrm{Al}_{2} \mathrm{Si}_{3} \mathrm{O}_{12}\right]\right)$, $\mathrm{Mn}, \mathrm{Fe}$ (= Almandinkomponente [ $\mathrm{Fe}_{3} \mathrm{Al}$ $\left.\left.{ }_{2} \mathrm{Si}_{3} \mathrm{O}_{12}\right]\right), \mathrm{Ca}$ (= Grossularkomponente $\left.\left[\mathrm{Ca}_{3} \mathrm{Al}_{2} \mathrm{Si}_{3} \mathrm{O}_{12}\right]\right)$ im untersuchten Mineral bildlich darzustellen. Man nennt diese Art der Darstellung Röntgenverteilungsbilder wie in Abb. 8B und 9B ersichtlich. Der Chemismus der Granate aus der monotonen Paragneis-Randzone des SC ist im Kern eher $\mathrm{Ca}$ (= grossular)-betont (Abb. 8B), während die ältere Granatgeneration (reliktische Granatkerne) im angrenzenden ÖC Ca-arm ist (Abb. 9B) und $\mathrm{Ca}$-reiche eo-Alpine Anwachssäume aufweisen (Abb. 9B). Habler et al. (2001) unterteilt in einer weiteren Untersuchung 
Kapitel 8 I Mineralogie und Petrologie des austroalpinen Kristallins
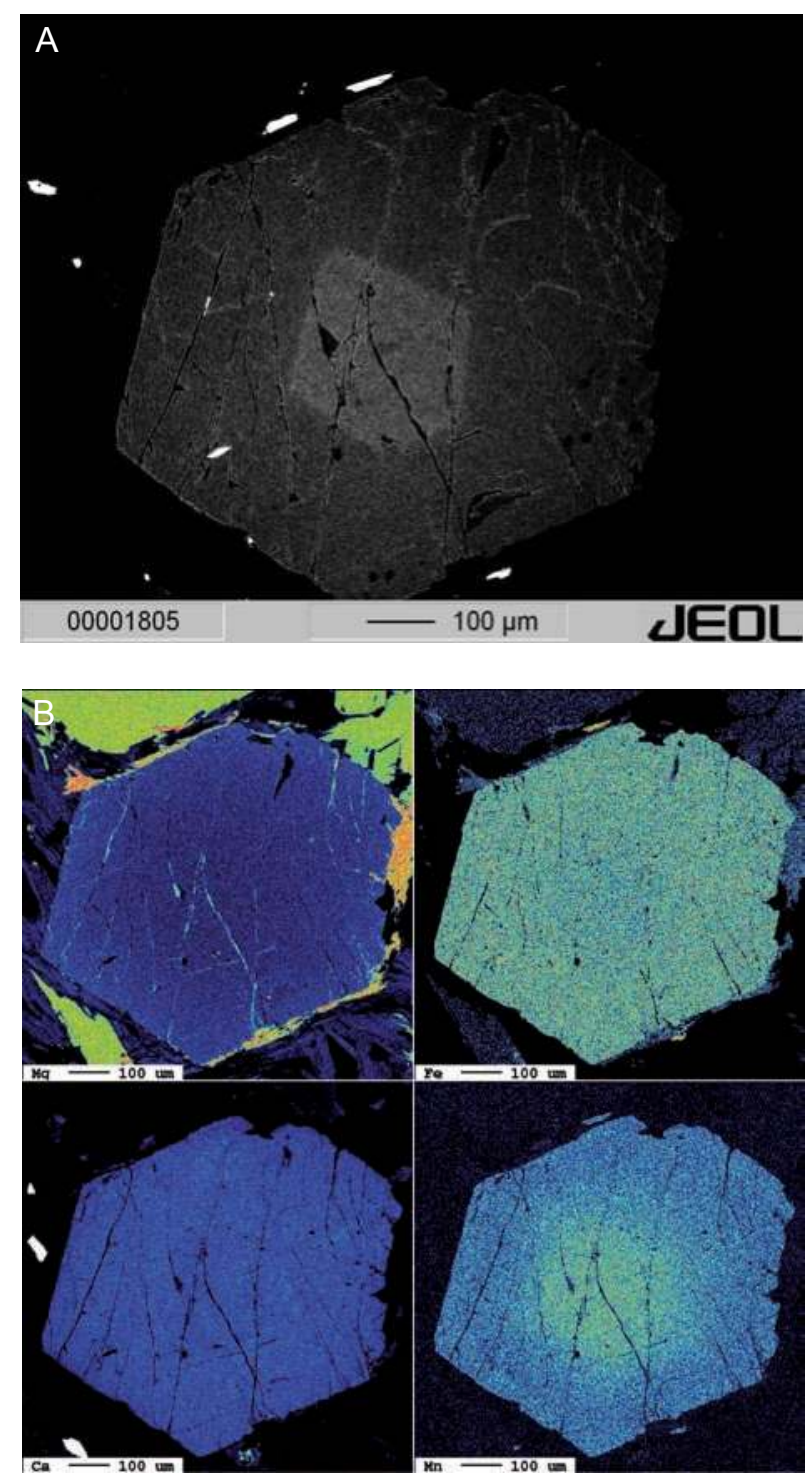

Abb. 8

Beispiel für eine chemische Granatzonierung (BSE: 8A; Röntgenverteilungsbild: 8B) aus dem Schneeberg Komplex (Probe aus den monotonen Paragneisen mit Biotitblastese). Die hellen Farben stehen für hohe und die dunklen Farben für niedrige Elementgehalte im Mineral. Deutlich sieht man ein kontinuierliches einphasiges Granatwachstum in der Probe aus dem Schneeberg Komplex. 

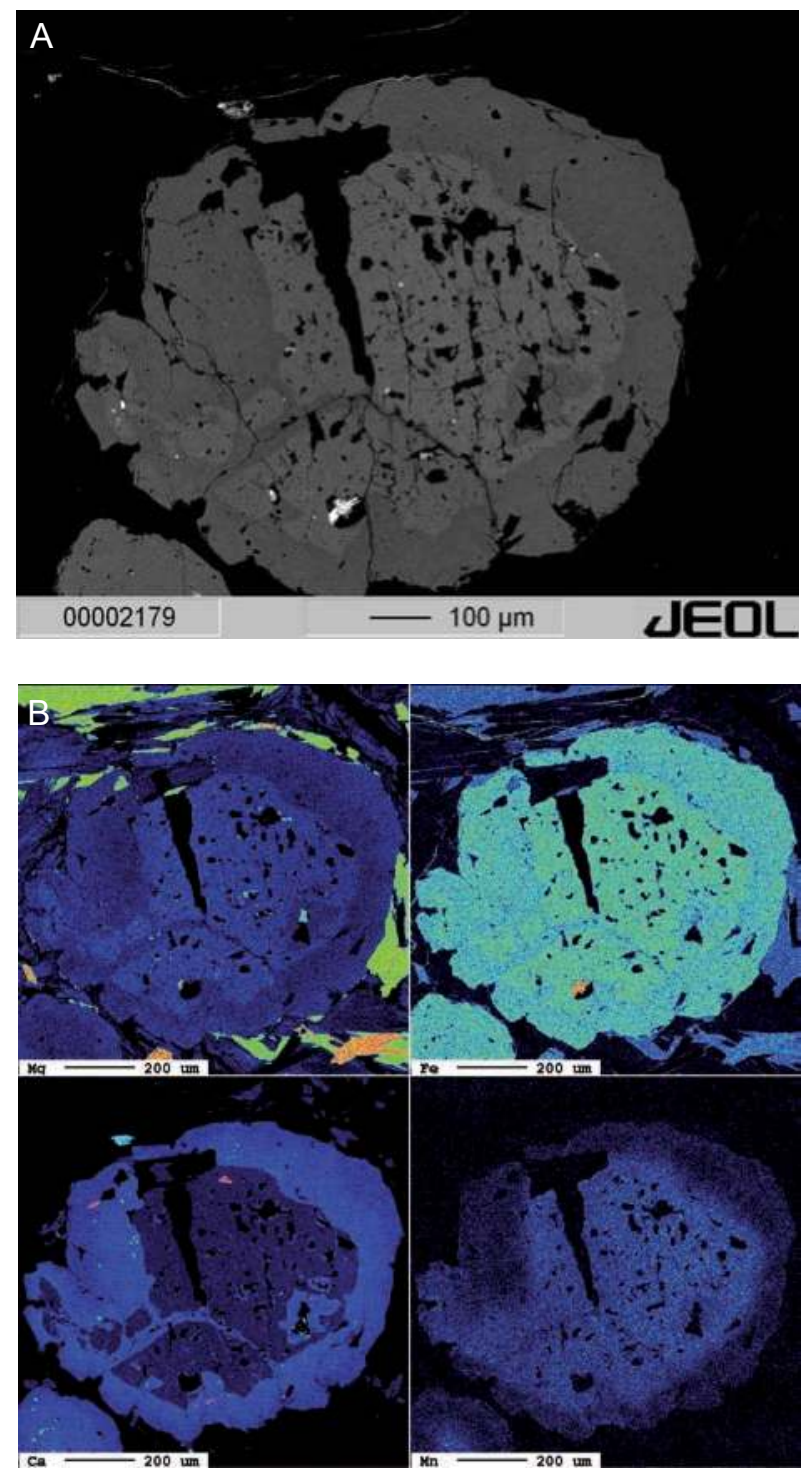

Abb. 9

Beispiel für eine chemische Granatzonierung (BSE: 9A: Röntgenverteilungsbild: 9B) aus den Paragneisen des ÖC. Deutlich sieht man ein diskontinuierliches zweiphasiges Wachstum in der Probe aus dem ÖC. 
das Granatwachstum der Al-reichen Lithologien der „Monotonen Serie“ im SC in drei Wachstumsphasen, wobei der MgGehalt kontinuierlich ansteigt und der Ca-Gehalt abnimmt. Al-arme Gesteine zeigen nur ein Granatwachstumsstadium.

\subsection{Hellglimmer}

Habler et al. (2001) identifizieren auch zwei Muskovitgenerationen $\left[\mathrm{KAl}_{2} \mathrm{Si}_{3} \mathrm{AlO}_{10}\left(\mathrm{OH}_{2}\right)\right]$ in den $\mathrm{Al}$-reichen Proben der „Monotonen Serie“, wobei die ältere Generation etwas Si-reicher (Si: 3.2-3.23 Si a.p.f.u., atoms per formula unit) als die Jüngere (Si: 3.15-3.18 Si a.p.f.u.) ist. Diese Werte decken sich auch mit den Muskovitanalysen von Tropper \& Recheis (2001) aus den Metapeliten des ÖC am Timmelsjoch im Hangenden der ÖC/SC Grenze. Konzett \& Hoinkes (1996) beschreiben Paragonit $\left[\mathrm{NaAl}_{2} \mathrm{Si}_{3} \mathrm{AlO}_{10}\left(\mathrm{OH}_{2}\right)\right]$ aus Amphiboliten des hinteren Seewertales. Die Analysen sind nahe der Endgliedzusammensetzung und die Anteile an der Margarit $\left[\mathrm{CaAl}_{2} \mathrm{Si}_{2} \mathrm{Al}_{2} \mathrm{O}_{10}\left(\mathrm{OH}_{2}\right)\right]$ - und Muskovitkomponente sind variabel mit 1.2-3.8 $\mathrm{Mol} . \%[=\mathrm{Ca} /(\mathrm{Na}+\mathrm{Ca}+\mathrm{K})]$ bzw. 3.816.9 Mol.\% $[=\mathrm{K} /(\mathrm{Na}+\mathrm{Ca}+\mathrm{K})]$. Paragonitkomponenten $[=\mathrm{Na} /(\mathrm{Na}+\mathrm{Ca}+\mathrm{K})]$ von 85 bis $91 \mathrm{Mol} \% \%$ wurden auch von Hoinkes (1981) aus den Metapeliten des SC beschrieben.

\subsection{Amphibol}

Bis dato gibt es nur chemische Daten bezüglich der Amphibole aus dem SC. Konzett \& Hoinkes (1996) beschrieben zwei Amphibolgenerationen, und zwar ältere $\mathrm{Mg}$-Hornblenden und Tschermakite $\left[\mathrm{Ca}_{2} \mathrm{Mg}_{3} \mathrm{Al}_{2} \mathrm{Si}_{6} \mathrm{Al}_{2} \mathrm{O}_{22}\left(\mathrm{OH}_{2}\right)\right]$, sowie jüngere aktinolithische Hornblenden bzw. Edenite aus den paragonitführenden Amphiboliten des Seewertales im SC. Die beiden Generationen unterscheiden sich in den Al Gehalten von 12-18 Gew. $\% \mathrm{Al}_{2} \mathrm{O}_{3}$ in der Älteren und 3-7 Gew. $\% \mathrm{Al}_{2} \mathrm{O}_{3}$ in der Jüngeren. In einer Probe konnte auch ein Mg-Amphibol, Cummingtonit $\left[\mathrm{Mg}_{7} \mathrm{Si}_{8} \mathrm{O}_{22}\left(\mathrm{OH}_{2}\right)\right]$, nachgewiesen werden. Dies stimmt mit den Beobachtungen von Hoinkes \& Mogessie (1986) aus Amphiboliten des SC aus dem Rotmoostal überein.

\subsection{Staurolith}

Hoinkes (1981) beschreibt die beginnende Staurolithbildung in den Gesteinen der „Monotonen Serie“. Die Staurolithe $\left[\left(\mathrm{Fe}^{2+}, \mathrm{Mg}, \mathrm{Zn}\right){ }_{2} \mathrm{Al}_{9}(\mathrm{Si}, \mathrm{Al}){ }_{4} \mathrm{O}_{22}(\mathrm{OH})_{2}\right]$ sind sehr Zn-reich (1.62-5.6 Gew\% $\mathrm{ZnO})$. Die Zn-Gehalte im Staurolith sind nicht vom $\mathrm{Zn}$-Gehalt des Gesamtgesteins (170-191 ppm ZnO) abhängig, sondern korrelieren mit dem Verteilungskoeffizienten von $\mathrm{Mg}$ und Fe zwischen Granat 
und Biotit und somit mit der Metamorphosetemperatur (Hoinkes 1981).

\section{Geothermobarometrie}

Die $P$-T Bedingungen der Metamorphose werden mittels Mineralreaktionen (Festkörperreaktionen, $\mathrm{Fe}-\mathrm{Mg}$ Austauschreaktionen), die zwischen den Mineralen im Gestein ablaufen, ermittelt. Hoinkes (1981; 1986a, b) ermittelte T-Bedingungen von $480-580^{\circ} \mathrm{C}$ bei $P$-Bedingungen von 6-6.5 kbar aus Metapeliten des SC für die eo-Alpine Metamorphose. Diese Temperaturen stimmen mit den Daten aus den Paragonit-führenden Amphiboliten der Monotonen Serie von Konzett \& Hoinkes (1996), den Metapeliten aus der „Monotonen Serie“ von Tropper \& Recheis (2003) und Habler et al. (2001) überein, die $P$-Werte sind jedoch niedriger. Die Untersuchungen von Konzett \& Hoinkes (1996), Tropper \& Recheis (2003) und Krenn (2010) ergaben Drucke im Bereich von 8-10 kbar. Ähnliche Metamorphosebedingungen konnten auch im angrenzenden ÖC von Tropper und Recheis (2003) im Bereich Schwarzseescharte und Egentenjoch, im Pflerschtal (Bernabe \& Tropper 2009) sowie dem Lazzacher Tal (Holzmann et al. 2010) nachgewiesen werden.

\section{Diskussion}

\subsection{Die Grenzziehung zwischen dem SC und ÖC entlang der Timmelsjoch Pass- straße}

Konzett et al. (2003) definieren nur einen ungefähren, mehrere Meter breiten Grenzbereich zwischen dem SC und dem ÖC, da die genaue Grenze im Gelände nicht festgestellt werden konnte. Pedevilla (2011) beprobte ein detailliertes Profil über diesen Grenzbereich entlang der Timmelsjoch Passstraße auf einer Höhe von ca. 2200 m. Es wurden 36 Proben genommen, welche mikroskopiert wurden. Vier ausgewählte Proben wurden an der Elektronenstrahlmikrosonde bezüglich ihrer Granatzonierungen untersucht. Die Proben aus dem SC (Monotone Serie, Bunte Randserie, Monotone Paragneise mit Biotitblastese; Abb. 8A, B) weisen alle einen einphasigen kontinuierlichen Zonarbau auf. Die monotonen Paragneise mit Biotitblastese wurden zwar schon von Schmidegg (1932) als „Grenzglimmerschiefer bzw. Biotitporphyroblastenschiefer" auskartiert, wurden jedoch zum ÖC gestellt. Erst das Auftreten von diskontinuierlich zonierten Granaten in feinkörnigen, monotonen Paragneisen (Abb. 9A, B), erlaubte eine Zuordnung zum ÖC (Pedevilla 2011). Erst diese mineralchemischen Daten, zusammen mit den petrographischen Daten ergeben dann die genaue Grenze zwischen dem 
SC und dem ÖC (Abb. 6), da der Geländebefund alleine keine sichere Grenzziehung zulässt.

\subsection{Tektonisches Modell des Austroal- pins (ÖC, SC, TC) westlich des Tauern- fensters}

Für den westlichen Bereich der Ostalpen gibt es mehrere tektonische Modelle (Sölva et al. 2005, Pomella et al. 2010, Krenn et al. 2011). Abb. 10A-C stellt die tektonische Entwicklung dieses Teils der Ostalpen ab der Oberkreide schematisch dar und basiert auf dem Modell von Krenn et al. (2011). Der SC befindet sich heute in einer Position zwischen dem ÖC im Hangenden und dem TC im Liegenden (Sölva et al. 2005). SC und TC werden nach Thöni \& Jagoutz (1993) zum eo-Alpinen Hochdruckgürtel bzw. nach Schmid et al. (2004) zum KoralpeWölz Hochdruck Deckensystem gezählt. Dieser Hochdruckgürtel repräsentiert einen während der eo-Alpinen Gebirgsbildung in der Kreidezeit herausgehobenen Krustenkeil („wedge“), der während der eo-Alpinen Metamorphose, die nach $S$ bis in die Eklogitfazies zunimmt, einer intensiven Deformation unterworfen wurde. Das ursprüngliche Alter des SC und dessen Position vor der eo-Alpinen Gebirgsbildung sind zurzeit noch unklar. Für die Metasedimente, die den SC im Wesentlichen aufbauen, wird aufgrund von lithologischen Vergleichen ein Paläo- zoisches Alter vermutet (Hoinkes et al. 1987) (Abb. 10A). Diese Sedimentgesteine überlagerten ein prä-Alpines Grundgebirge (Basement), welches zumindest eine vor-Alpidische Metamorphose (Permisches Hochtemperaturereignis) oder auch noch eine ältere Metamorphose (Variszisches druckbetontes Ereignis) erlebt hatte. Gemeinsam mit der sedimentären Bedeckung (zukünftiger SC) wurde dieses prä-Alpine Basement in der frühen Kreidezeit vor ca. 90-80 Ma in Tiefen von 35$40 \mathrm{~km}$ subduziert (Abb. 10B). Als Teil des eo-Alpinen Hochdruckgürtels wurden SC und TC während der Hebung intensiv verfaltet und von der Oberplatte, die den ÖC repräsentiert, überfahren. Aufgrund dieses kontinuierlichen Prozesses wurden auch basale Teile der Oberplatte in den Hochdruckgürtel mit eingefaltet (Abb. 10B). Der SC selbst kann am besten als eine großräumige Faltenstruktur beschrieben werden, der sich aufgrund späterer Erosion als ein System mehrerer geschlossener Synformen zu erkennen gibt (Abb. 10C). Umrahmt werden diese Synformen von Gesteinen des TC und ÖC, wobei als Resultat dieser intensiven Verfaltung um steile Achsen auch Gesteine des TC innerhalb des ÖC anzutreffen sind und so den „Schlingenbau“ in der geologischen Karte von Schmidegg (1932) verdeutlichen. Die Gesteine im Übergangsbereich ÖC-SCTC fallen durchwegs nach NW ein und passen nicht ins Gesamtbild einer nach $\mathrm{N}$ bzw. NW gerichteten Deckenstapelung, die den gesamten Ostalpenraum erfasste. 


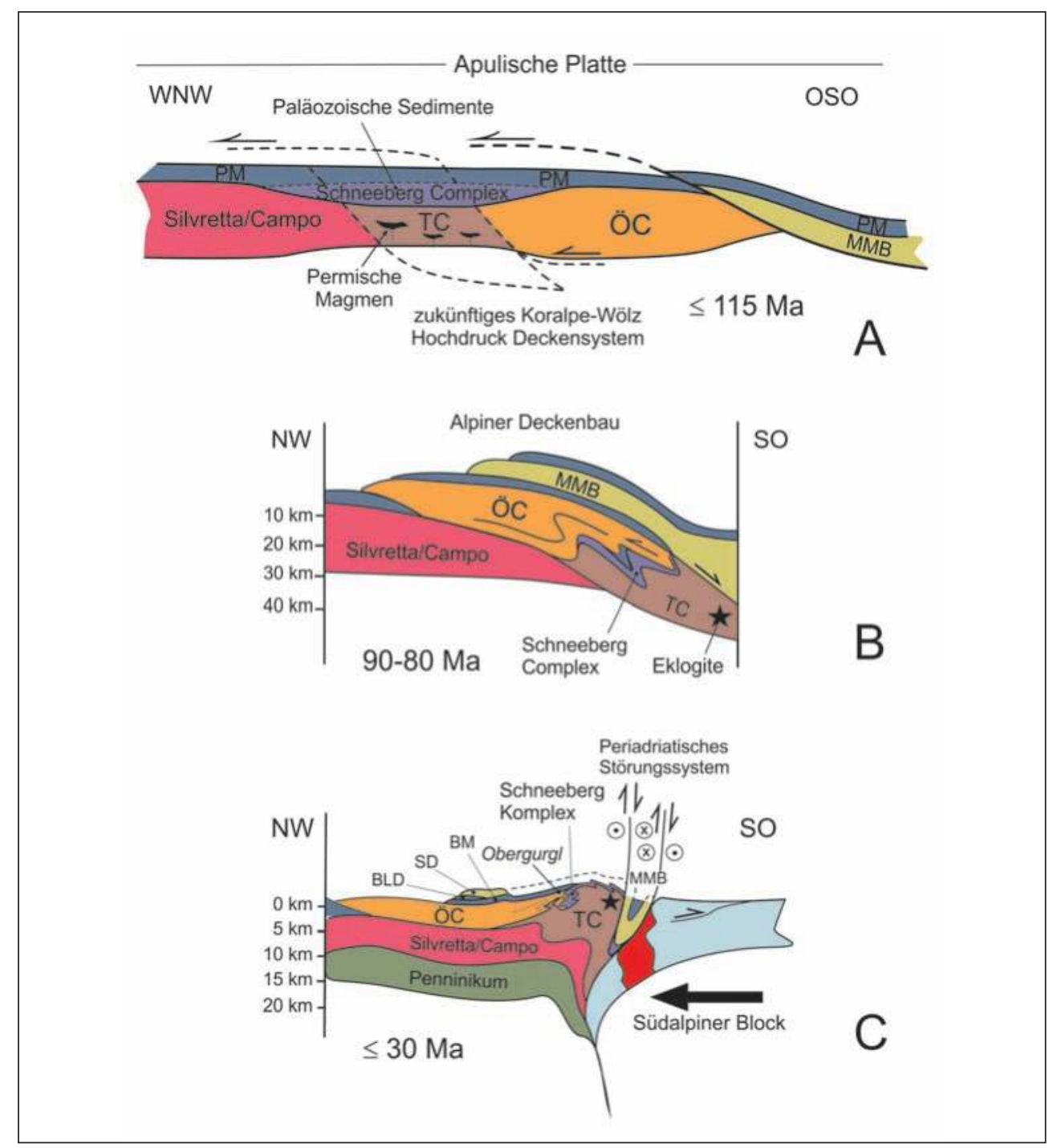

Abb. 10

Vereinfachte Darstellung der geodynamischen Entwicklung von ÖC, SC und TC, während der prä-Alpinen (A), der eo-Alpinen (B: Subduktion mit anschließender Exhumation) und der Alpinen (C: Indentertektonik) Orogenese nach Krenn et al. (2011). Die Skala auf der linken Seite gibt die Mächtigkeiten des Deckenstapels in $\mathrm{km}$ an. Die Pfeile in den Abbildungen A und C implizieren die Bewegungsrichtungen entlang von Störungen. Die Kreise in Abbildung $C$ weisen auf Bewegungen entlang von Störungen in Richtung des Betrachters (Punkt) als auch vom Betrachter weg (Kreuz) hin. Abkürzungen: BLD: Blaser Decke; SD: Steinacher Decke; BM: Brenner Mesozoikum; MMB: Meran-Mauls Basement; ÖC: Ötztal Komplex; TC: Texel Komplex; PM: Permo-Mesozoikum; Ma: Millionen Jahre 
Es wird daher vermutet, dass dieser Bereich des eo-Alpinen Hochdruckgürtels westlich des Tauernfensters während der Bildung des südlich gelegenen Periadriatischen Störungssystems verkippt wurde (Abb. 10C). Dies wird während der NWSO Einengung durch die miozäne NWBewegung des südalpinen Intenderblocks verwirklicht. Die Gesteine des SC, des TC sowie auch die angrenzende Basis des ÖC wurden daher von einer NW-gerichteten Überschiebungsgeometrie in ein steiles nach NW einfallendes Gesteinspaket übergeführt. Diese Aufsteilung und Verkippung führte dazu, dass der eo-Alpine Metamorphosegradient im ÖC nun an der Oberfläche ersichtlich ist.

\section{Literatur}

Ampferer, O. (1906) Über das Bewegungsbild von Faltengebirgen. Jahrbuch der Geologischen Reichsanstalt 56: 539-622.

Bernabe, E., Tropper, P. (2009) The metamorphic evolution and thermobarometry of amphibolites from the Pflersch-metadiorite complex (Pflersch Valley, SouthTyrol, Italy). Mitteilungen der Österreichischen Mineralogischen Gesellschaft 155: 32 .

Bernhard, F. (1994) Zur magmatischen und metamorphen Entwicklung im westlichen Ötztal-Stubai Kristallin (Bereich Feichten-Verpeil, mittleres Kaunertal).
Unveröffentlichte Diplomarbeit, Universität Graz, 314 S.

Fügenschuh, B. (1995) Thermal and kinematic history of the Brenner area (Eastern Alps, Tyrol). Unveröffentlichte Dissertation, ETH Zürich.

Habler, G., Linner, M., Thiede, R., Thöni, M. (2001) Eo-Alpine Andalusite in the Schneeberg Complex (Eastern Alps, Italy/Austria): Constraining the P-T-t-D Path during Cretaceous Metamorphism. Journal of Conference Abstracts 6: 340. Habler, G., Thöni, M., Miller, C., Schuster, R. (2005) Grt Sm-Nd and mineral chemical data of meta-pegmatites and their metapelitic host rock: relating PermoTriassic magmatic events to the polymetmorphic evolution of Austroalpine basement units (Eastern Alps). Geophysical Research Abstracts 7, 09060.

Habler, G., Thöni, M., Sölva, H. (2006) Tracing the high pressure stage in the polymetamorphic Texel Complex (Austroalpine basement unit, Eastern Alps): P-T-t-d constraints. Mineralogy and Petrology 88: 269-296.

Hoinkes, G., Purtscheller, F., Schantl, J. (1972) Zur Petrographie und Genese des Winnebachgranites (Ötztaler Alpen, Tirol). Tschermaks Mineralogische und Petrographische Mitteilungen 18: 292311.

Hoinkes, G. (1973) Die Anatexis des Winnebachgranites (Ötztaler Alpen, Österreich) am Beispiel eines Aufschlusses. Tschermaks Mineralogische und Petrographische Mitteilungen 20: 225-239. 
Hoinkes, G. (1981) Mineralreaktionen und Metamorphosebedingungen in Metapeliten des westlichen Schneebergerzuges und des angrenzenden Altkristallins (Ötztaler Alpen). Tschermaks Mineralogisch Petrographische Mitteilungen 28: 31-54.

Hoinkes, G., Purtscheller, F., Tessadri, R. (1982) Polymetamorphose im Ostalpin westlich der Tauern (Ötztaler Masse, Schneeberger Zug, Brennermesozoikum). Geologisch-Paläontologische Mitteilungen der Universität Innsbruck 12: 95-113.

Hoinkes, G. (1983) Cretaceous metamorphism of metacarbonates in the Austroalpine Schneeberg complex, Tirol. Schweizerische Mineralogische und Petrographische Mitteilungen 63: 95-114.

Hoinkes, G., Mogessie, M. (1986) Coexisting cummingtonite and calcic amphibole in amphibolites from the Schneeberg Complex, Tyrol, Austria. Tschermaks Mineralogische und Petrographische Mitteilungen 35: 33-45.

Hoinkes, G. (1986a) Eoalpine metamorphism of the Austroalpine Schneeberg-Complex and the adjacent Ötztal crystalline basement (summary). Schweizerische Mineralogische und Petrographische Mitteilungen 66: 135-138.

Hoinkes, G. (1986b) Effect of grossularcontent in garnet on the partitioning of Fe and $\mathrm{Mg}$ between garnet and biotite: An empirical investigation on staurolite-zone samples from the Austroalpine
Schneeberg Complex. Contributions to Mineralogy and Petrology 92: 393-399.

Hoinkes, G., Frank, W., Mauracher, J., Peschel, R., Purtscheller, F., Tessadri, R. (1987) Petrography of the Schneeberg Complex. In: Geodynamics of the Eastern Alps, Flügel, H.W., Faupl, P. (Hg.), Deuticke, Wien: 190-199.

Hoinkes, G., Kostner, A., Thöni, M. (1991) Petrologic constraints for Eoalpine eclogite facies metamorphism in the Austroalpine Ötztal basement. Mineralogy and Petrology 43: 237-254.

Hoinkes, G., Thöni, M. (1993) Evolution of the Ötztal-Stubai, Scarl-Campo and Ulten basement units. In: Premesozoic geology in the Alps, Raumer, J. F. \& Neubauer, J.F. (Hg.), Springer Verlag: 485-494.

Hoinkes, G., Thöni, M., Bernhard, F., Kaindl, R., Lichem, C., Schweigl, J., Tropper, P., Cosca, M. (1997) Metagranitoids and associated metasediments as indicators for the pre-Alpine magmatic and metamorphic evolution of the western Austroalpine Ötztal Basement (Kaunertal, Tirol). Schweizerische Mineralogische und Petrographische Mitteilungen 77: 299-314.

Hoinkes, G., Koller, F., Rantitsch, G., Dachs, E., Höck, V., Neubauer, F., Schuster, R. (1999) Alpine Metamorphism of the Eastern Alps. Schweizerische Mineralogische und Petrographische Mitteilungen 79: 155-181. 
Holzmann, J., Tropper, P., Mair, V., Vavtar, F. (2010) Petrology of the silicate/ore contact zone of the $\mathrm{Pb}-\mathrm{Zn}$ deposit Pflersch/ Schneeberg (South-Tyrol, Italy). PANGEO 2010 Abstracts, Journal of Alpine Geology 52: 144-145.

Klötzli-Chowanetz, E., Klötzli, U., Koller, F. (1997) Lower Ordovician migmatisation in the Ötztal crystalline basement (Eastern Alps, Austria): linking U-Pb and $\mathrm{Pb}-\mathrm{Pb}$ dating with zircon morphology. Schweizerische Mineralogische und Petrographische Mitteilungen 77: 315-324.

Konzett, J., Hoinkes, G. (1996) Paragonitehornblende assemblages and petrological significance: an example from the Austroalpine Schneeberg Complex, Southern Tyrol, Italy. Journal of Metamorphic Geology 14: 85-101.

Konzett, J., Hoinkes, G., Tropper, P. (2003) $5^{\text {th }}$ Workshop of Alpine geological studies. Field trip guide E4: Alpine metamorphism in the Schneeberg Complex and neighboring units (immediate vicinity of Obergurgl). Geologisch Paläontologische Mitteilungen Innsbruck 26: 21-45.

Krenn, K. (2010) Fluid inclusions related to subsequent stages of foliation development during a single metamorphic cycle (Schneeberg Fault Zone, Eastern Alps, Austria). Lithos 118: 255-268.

Krenn, K., Kurz, W., Fritz, H., Hoinkes, G. (2011) Eoalpine tectonics of the Eastern Alps: implication from the evolution of monometamorphosed Austroalpine units (Schneeberg- and Radenthein
Complex), Swiss Journal of Geoscience, DOI: 10.1007/s00015-011-0087-8.

Mauracher, J. (1980) Alpidische und voralpidische Metamorphose und Strukturprägung am Westende des Schneeberger Zuges (Ötztaler Alpen). Unveröffentlichte Dissertation, Universität Wien, 155 S.

Miller, C., Thöni, M., (1995) Origin of eclogites from the Austroalpine Ötztal basement (Tyrol, Austria): geochemistry and $\mathrm{Sm}-\mathrm{Nd}$ vs. Rb-Sr isotope systematics. Chemical Geology 122: 199-225.

Pedevilla, A. (2011) Petrographische Untersuchungen des Übergangs Ötztal-Stubai Kristallin-Schneeberger Zug im Bereich des Timmelsjoches. Unveröffentlichte Bachelorarbeit, Universität Innsbruck, $53 \mathrm{~S}$.

Pomella, H., Flöss, D., Speckbacher, R., Fügenschuh, B. (2010) The evolution of the Austroalpine nappe stack in the hanging wall of the Guidicaric fault system. Pangeo 2010, Journal of Alpine Geology, 72, 202.

Proyer, A. (1989) Petrologie der Rahmengesteine der $\mathrm{Pb}-\mathrm{Zn}$ Lagerstätte Schneeberg, Südtirol. Unveröffentlichte Diplomarbeit, Universität Innsbruck, 103 S.

Purtscheller, F. (1969) Petrographische Untersuchungen an Aluminosilikatgneisen des Ötztaler-Stubaier Altkristallins. Tschermaks Mineralogisch Petrographische Mitteilungen 13: 35-54.

Purtscheller, F., Rammlmair, D. (1982) Alpine metamorphism of diabase dikes in the Ötztal-Stubai metamorphic complex. 
Tschermaks Mineralogisch Petrographische Mitteilungen 29: 205-221.

Purtscheller, F., Haas, R., Hoinkes, G., Mogessie, A., Tessadri, R., Veltman, C. (1987) Eoalpine metamorphism in the crystalline basement. In: Geodynamics of the Eastern Alps. Flügel, H.W., Faupl, P. (Hg.), Deuticke, Wien: 187-189.

Schmid, S., Fügenschuh, B., Kissling, E., Schuster, R. (2004) Tectonic map and overall architecture of the Alpine orogen. Eclogae Geologae Helveticae 97: 93-117.

Schmidegg, O. (1932) Geologische Spezialkarte der Republik Österreich, Blatt Sölden und St. Leonhard. Geologische Bundesanstalt Wien 1932.

Schmidegg, O. (1936) Steilachsige Tektonik und Schlingenbau auf der Südseite der Tiroler Zentralalpen. Jahrbuch der Geologischen Bundesanstalt, 70, Heft 3 und 4: 325-334.

Schmidegg, O. (1964) Die Ötztaler Schubmasse und ihre Umgebung. Verhandlungen der Geologischen Bundesanstalt 1: $27-47$.

Schweigl, J. (1995) Neue geochronologische und isotopengeologische Daten zur voralpidischen Entwicklungsgeschichte im Ötztalkristallin (Ostalpen). Jahrbuch der Geologischen Bundesanstalt 138: 131149.

Söllner, F., Hansen, B. T. (1987) „Pan-afrikanisches" und "kaledonisches" Ereignis im Ötztal-Kristallin der Ostalpen: RbSr- und U-Pb-Altersbestimmungen an Migmatiten und Metamorphiten. Jahr- buch der Geologischen Bundesanstalt 130/4: 529-569.

Söllner, F. (2001) The Winnebach migmatite (Ötz-Stubai crystalline unit) - evidence for a Pan-Africanic metamorphism in an overthrust nappe sequence in the Eastern Alps. Geologisch Paläontologische Mitteilungen der Universität Innsbruck 25: 199-200.

Sölva, H., Grasemann, B., Thöni, M., Thiede, R., Habler, G. (2005) The Schneeberg Normal Fault Zone: normal faulting associated with Cretaceous SE-directed extrusion in the Eastern Alps (Italy/Austria). Tectonophysics 401: 143-166.

Stampfli, G.M., Borel, G., Cavazza, W., Mosar, J., Ziegler, P.A. (2001) The paleotectonic atlas of the Peritethyan domain. CD ROM, European Geophysical Society.

Thöni, M. (1981) Degree and evolution of the Alpine metamorphism in the Austroalpine unit $\mathrm{W}$ of the Hohe Tauern in the light of $\mathrm{K} / \mathrm{Ar}$ and $\mathrm{Rb} / \mathrm{Sr}$ age determinations on micas. Jahrbuch der geologischen Bundesanstalt 124: 111-174.

Thöni, M., Hoinkes, G. (1987) The Southern Ötztal Basement: Geochronological and Petrological Consequences of Eoalpine Metamorphic Overprinting. In: Geodynamics of the Eastern Alps, Flügel, H.W. \& Faupl, P. (Hg.), Deuticke, Wien: 379406.

Thöni, M., Jagoutz, E. (1993) Isotopic constraints for Eo-Alpine high-P metamorphism in the Austroalpine nappes of the Eastern Alps: bearing on Alpine orogeni- 
sis. Schweizerische Mineralogische und Petrographische Mitteilungen 73: $177-$ 189.

Thöni, M. (1999) A review of geochronological data from the Eastern Alps. Schweizerische Mineralogische und Petrographische Mitteilungen 79: 209-230.

Thöni, M. (2006) Dating eclogite-facies metamorphism in the Eastern Alps approaches, results, interpretations: a review. Mineralogy and Petrology 88: 123-148.

Thöny, W., Tropper, P., Schennach, F., Krenn, E., Finger, F., Kaindl, R., Bernhard, F., Hoinkes, G. (2008) The metamorphic evolution of migmatites from the Ötztal Complex (Tyrol, Austria) and constraints on the timing of the pre-Variscan high-T event in the Eastern Alps. Swiss Journal of Geosciences 101: 111-126.

Tropper, P., Hoinkes, G. (1996) Geothermobarometry of $\mathrm{Al}_{2} \mathrm{SiO}_{5}$-bearing metapelites in the western Austroalpine Ötztalbasement. Mineralogy and Petrology 58: 145-170.

Tropper, P., Recheis, A. (2003) Garnet zoning as a window into the metamorphic evolution of a crystalline complex: the northern and central Austroalpine Ötztalbasement as a polymetamorphic example. Mitteilungen der Österreichischen Geologischen Gesellschaft 94: 27-53.

Van Gool, J.A.M., Kemme, M.M.J., Schreurs, G.M.M.F. (1987) Structural investiga- tions along an E-W cross-section in the southern Ötztal Alps. In: Geodynamics of the Eastern Alps, Flügel, H.W., Faupl, P. (Hg.), Deuticke, Wien 1987: 214222.

Wegener, A. (1912) Die Entstehung der Kontinente. Petermanns Geographische Mitteilungen: 185-308.

\section{Verzeichnis der Autoren}

\section{Peter Tropper}

Institut für Mineralogie und Petrographie

Universität Innsbruck

Innrain 52f, 6020 Innsbruck

Peter.Tropper@uibk.ac.at

\section{Kurt Krenn}

Institut für Erdwissenschaften

Universität Graz

Universitätsplatz 2, $8010 \mathrm{Graz}$

kurt.krenn@uni-graz.at

Georg Hoinkes

Institut für Erdwissenschaften

Universität Graz

Universitätsplatz 2, $8010 \mathrm{Graz}$

georg.hoinkes@uni-graz.at 


\section{R. Mayer, F. Nagl, B. Erschbamer}

\section{Subalpine Wiesen und Weiden - die Kulturlandschaft der subalpinen Stufe}

Tab. A1: Stetigkeitstabelle der subalpinen Wiesen und Weiden im Untersuchungsgebiet nach dem Ergebnis der TWINSPAN-Analyse. Folgende Subassoziationen wurden unterschieden:

1: Sieversio-Nardetum strictae vaccinietosum

2: Sieversio-Nardetum strictae typicum

3: Sieversio-Nardetum strictae trifolietosum pratensis

4: Trisetetum flavescentis nardetosum

5: Trisetetum flavescentis typicum

D: Differenzialarten, C: Charakterarten.

\begin{tabular}{lc|c|c|c|c}
\hline & \multicolumn{3}{c}{ Sieversio-Nardetum strictae } & \multicolumn{2}{c}{$\begin{array}{c}\text { Trisetetum } \\
\text { flavescentis }\end{array}$} \\
\hline Assoziation & 1 & 2 & 3 & 4 & 5 \\
\hline Subassoziation Nr. & 20 & 8 & 21 & 32 & 10 \\
\hline Anzahl der Aufnahmen & 2056 & 1978 & 1998 & 1966 & 1879 \\
\hline Mittlere Meereshöhe (m) & 23,0 & 27,5 & 23,7 & 20,7 & 3,6 \\
Mittlere Neigung ( ${ }^{\circ}$ ) & 4,11 & 4,15 & 4,23 & 4,41 & 4,61 \\
Mittlerer pH-Wert des Bodens & 99 & 103 & 115 & 132 & 108 \\
Mittlerer Hitze-Index & 24,0 & 3,5 & 1,3 & 0,0 & 0,0 \\
\hline Mittlerer Anteil Zwergsträucher (\%) & 38,8 & 49,9 & 46,7 & 43,9 & 38,7 \\
Mittlerer Anteil Grasartige (\%) & 33,7 & 41,5 & 44,7 & 44,8 & 46,3 \\
Mittlerer Anteil Kräuter (\%) & 3,6 & 5,1 & 7,2 & 11,3 & 15,1 \\
Mittlerer Anteil Leguminosen (\%) & 40 & 39 & 36 & 26 & 16 \\
\hline Mittlere Artenzahl je Aufnahme & 2,95 & 2,90 & 2,92 & 2,63 & 2,30 \\
Mittlerer Shannon-Index je Aufnahme & 0,0 & 0,0 & 0,0 & 0,0 & 70,0 \\
\hline Anteil 2-schürige Mahd (\%) & 0,0 & 12,5 & 19,1 & 79,3 & 30,0 \\
Anteil 1-schürige Mahd (\%) & 0,0 & 0,0 & 14,3 & 0,0 & 0,0 \\
Anteil halbschürige Mahd (\%) & 35,0 & 62,5 & 33,3 & 0,0 & 0,0 \\
Anteil Beweidung (\%) & 10,0 & 0,0 & 4,8 & 13,8 & 0,0 \\
Anteil Brache jung (\%) & 55,0 & 25,0 & 28,6 & 6,9 & 0,0 \\
Anteil Brache alt (\%) & & & &
\end{tabular}




\begin{tabular}{|c|c|c|c|c|c|c|}
\hline \multirow{2}{*}{\multicolumn{2}{|c|}{$\begin{array}{l}\text { Assoziation } \\
\text { Subassoziation } \mathrm{Nr} \text {. }\end{array}$}} & \multicolumn{3}{|c|}{ Sieversio-Nardetum strictae } & \multicolumn{2}{|c|}{$\begin{array}{l}\text { Trisetetum } \\
\text { flavescentis }\end{array}$} \\
\hline & & 1 & 2 & 3 & 4 & 5 \\
\hline \multicolumn{2}{|c|}{ Anteil Düngung mit Mist (\%) } & 0,0 & 12,5 & 28,6 & 62,1 & 100,0 \\
\hline \multicolumn{2}{|c|}{ Anteil Düngung mit Mineraldünger (\%) } & 0,0 & 12,5 & 23,8 & 55,2 & 30,0 \\
\hline \multirow[t]{9}{*}{ D1 } & Vaccinium vitis-idaea & $\mathrm{V}$ & II & I & . & . \\
\hline & Potentilla erecta & $\mathrm{V}$ & III & III & . & . \\
\hline & Vaccinium gaultherioides & IV & II & I & . & . \\
\hline & Pedicularis tuberosa & III & . & . & . & . \\
\hline & Gymnadenia conopsea & III & . & I & . & . \\
\hline & Antennaria dioica & III & I & . & . & . \\
\hline & Arnica montana & III & I & I & . & . \\
\hline & Juncus trifidus & III & I & I & I & . \\
\hline & Scabiosa columbaria & II & . & . & . & . \\
\hline \multirow[t]{2}{*}{ D2 } & Hieracium pilosella & II & $\mathrm{V}$ & I & . & . \\
\hline & Veronica officinalis & . & II & . & . & . \\
\hline \multirow[t]{8}{*}{ D5 } & Phleum pratense & . & . & . & I & $\mathrm{V}$ \\
\hline & Taraxacum Sect. Ruderalia & . & . & . & II & $\mathrm{V}$ \\
\hline & Poa trivialis & . & . & I & III & $\mathrm{V}$ \\
\hline & Trifolium repens & . & III & II & III & $\mathrm{V}$ \\
\hline & Poa pratensis & . & I & . & I & IV \\
\hline & Carum carvi & . & I & . & $\mathrm{I}$ & IV \\
\hline & Elymus repens & . & . & . & . & III \\
\hline & Trifolium pratense ssp. pratense & . & . & . & I & III \\
\hline \multirow[t]{7}{*}{ D1-D3 } & Carex sempervirens & $\mathrm{V}$ & $\mathrm{V}$ & $\mathrm{V}$ & $\mathrm{I}$ & . \\
\hline & Nardus stricta & $\mathrm{V}$ & $\mathrm{V}$ & V & III & . \\
\hline & Lotus corniculatus & $\mathrm{V}$ & IV & $\mathrm{V}$ & $\mathrm{I}$ & $\mathrm{I}$ \\
\hline & Luzula multiflora & $\mathrm{V}$ & IV & $\mathrm{V}$ & II & . \\
\hline & Silene nutans & IV & IV & III & I & . \\
\hline & Briza media & III & $\mathrm{V}$ & IV & I & . \\
\hline & Carlina acaulis & III & IV & III & I & . \\
\hline
\end{tabular}




\begin{tabular}{|c|c|c|c|c|c|}
\hline \multirow{2}{*}{$\begin{array}{l}\text { Assoziation } \\
\text { Subassoziation Nr. }\end{array}$} & \multicolumn{3}{|c|}{ Sieversio-Nardetum strictae } & \multicolumn{2}{|c|}{$\begin{array}{l}\text { Trisetetum } \\
\text { flavescentis }\end{array}$} \\
\hline & 1 & 2 & 3 & 4 & 5 \\
\hline \multicolumn{6}{|l|}{ C Caricetea curvulae } \\
\hline Potentilla aurea & $\mathrm{V}$ & $\mathrm{V}$ & $\mathrm{V}$ & IV & . \\
\hline Avenula versicolor & IV & . & I & . & . \\
\hline Gentiana acaulis & IV & II & I & . & . \\
\hline Trifolium alpinum & III & II & . & . & . \\
\hline \multicolumn{6}{|l|}{ C Festucetalia spadiceae } \\
\hline Phyteuma betonicifolium & IV & $\mathrm{V}$ & IV & IV & I \\
\hline Campanula barbata & $\mathrm{V}$ & II & III & I & . \\
\hline Geum montanum & IV & IV & III & I & . \\
\hline \multicolumn{6}{|l|}{ C Nardion strictae } \\
\hline Ajuga pyramidalis & I & II & I & . & . \\
\hline Pseudorchis albida & $\mathrm{I}$ & . & $\mathrm{I}$ & . & . \\
\hline \multicolumn{6}{|l|}{ C Molinio-Arrhenatheretea } \\
\hline Alchemilla vulgaris agg. & II & IV & $\mathrm{V}$ & $\mathrm{V}$ & $\mathrm{V}$ \\
\hline Achillea millefolium agg. & II & $\mathrm{V}$ & IV & IV & IV \\
\hline Leontodon hispidus & III & III & $\mathrm{V}$ & IV & II \\
\hline Ranunculus acris & I & IV & IV & $\mathrm{V}$ & $\mathrm{V}$ \\
\hline Deschampsia cespitosa & . & II & IV & IV & III \\
\hline \multicolumn{6}{|l|}{ C Poo alpinae Trisetetalia } \\
\hline Agrostis capillaris & $\mathrm{V}$ & $\mathrm{V}$ & $\mathrm{V}$ & $\mathrm{V}$ & IV \\
\hline Rumex alpestris & $\mathrm{I}$ & IV & $\mathrm{V}$ & $\mathrm{V}$ & $\mathrm{V}$ \\
\hline Campanula scheuchzeri & III & $\mathrm{V}$ & $\mathrm{V}$ & IV & I \\
\hline Poa alpina & . & II & I & III & I \\
\hline \multicolumn{6}{|l|}{ C Polygono-Trisetion } \\
\hline Trollius europaeus & I & . & II & I & . \\
\hline Crocus albiflorus & . & . & I & I & . \\
\hline Viola tricolor & . & . & I & . & I \\
\hline \multicolumn{6}{|l|}{ Begleiter } \\
\hline Festuca nigrescens & $\mathrm{V}$ & $\mathrm{V}$ & $\mathrm{V}$ & $\mathrm{V}$ & II \\
\hline Anthoxanthum alpinum & IV & $\mathrm{V}$ & $\mathrm{V}$ & $\mathrm{V}$ & II \\
\hline
\end{tabular}




\begin{tabular}{|c|c|c|c|c|c|}
\hline \multirow{2}{*}{$\begin{array}{l}\text { Assoziation } \\
\text { Subassoziation Nr. }\end{array}$} & \multicolumn{3}{|c|}{ Sieversio-Nardetum strictae } & \multicolumn{2}{|c|}{$\begin{array}{l}\text { Trisetetum } \\
\text { flavescentis }\end{array}$} \\
\hline & 1 & 2 & 3 & 4 & 5 \\
\hline Myosotis alpestris & III & III & IV & IV & III \\
\hline Rhinanthus glacialis & III & II & III & IV & II \\
\hline Chaerophyllum villarsii & III & IV & V & IV & I \\
\hline Silene vulgaris & II & IV & $\mathrm{V}$ & IV & I \\
\hline Geranium sylvaticum & I & III & IV & IV & II \\
\hline Phleum rhaeticum & I & II & $\mathrm{V}$ & V & I \\
\hline Trifolium pratense ssp. nivale & IV & $\mathrm{V}$ & $\mathrm{V}$ & $\mathrm{V}$ & . \\
\hline Ranunculus montanus & IV & IV & IV & III & . \\
\hline $\begin{array}{l}\text { Euphrasia officinalis ssp. } \\
\text { rostkoviana }\end{array}$ & III & II & III & II & . \\
\hline Galium anisophyllon & III & IV & IV & II & . \\
\hline Cerastium holosteoides & I & II & II & II & . \\
\hline Laserpitium halleri & V & $\mathrm{V}$ & II & I & . \\
\hline Solidago virgaurea & III & IV & I & I & . \\
\hline Thymus pulegioides & III & IV & I & I & . \\
\hline Pulsatilla alpina ssp. apiifolia & III & II & II & I & . \\
\hline Persicaria vivipara & III & I & II & I & . \\
\hline Veronica fruticans & II & II & II & I & . \\
\hline Trifolium badium & I & II & II & II & . \\
\hline Crepis conyzifolia & I & II & II & I & . \\
\hline Juncus jacquinii & I & II & II & I & . \\
\hline Cerastium arvense ssp. strictum & I & II & I & I & . \\
\hline Carduus defloratus & I & II & I & I & . \\
\hline Luzula luzuloides & I & II & I & I & . \\
\hline Sempervivum montanum & I & I & I & I & . \\
\hline Calluna vulgaris & $\mathrm{V}$ & IV & I & . & . \\
\hline Vaccinium myrtillus & $\mathrm{V}$ & IV & II & . & . \\
\hline Avenella flexuosa & IV & III & II & . & . \\
\hline Juniperus communis ssp. nana & IV & IV & I & . & . \\
\hline Thesium alpinum & III & II & I & . & . \\
\hline Helianthemum grandiflorum & II & I & I & . & . \\
\hline
\end{tabular}




\begin{tabular}{|c|c|c|c|c|c|}
\hline \multirow{2}{*}{$\begin{array}{l}\text { Assoziation } \\
\text { Subassoziation Nr. }\end{array}$} & \multicolumn{3}{|c|}{ Sieversio-Nardetum strictae } & \multicolumn{2}{|c|}{$\begin{array}{l}\text { Trisetetum } \\
\text { flavescentis }\end{array}$} \\
\hline & 1 & 2 & 3 & 4 & 5 \\
\hline Potentilla grandiflora & I & II & I & . & . \\
\hline Prunella vulgaris & II & II & I & . & . \\
\hline Homogyne alpina & II & I & I & . & . \\
\hline Carex ornithopoda & I & II & I & . & . \\
\hline Gentianella germanica agg. & II & II & I & . & . \\
\hline Nigritella rhellicanii & II & II & . & I & . \\
\hline Poa chaixii & I & I & I & . & . \\
\hline Atocion rupestre & I & I & I & . & . \\
\hline Euphrasia minima & II & I & . & . & . \\
\hline Parnassia palustris & I & I & . & . & . \\
\hline Plantago strictissima & I & $\mathrm{I}$ & . & . & . \\
\hline Festuca halleri & I & I & . & . & . \\
\hline Veronica bellidioides & I & $\mathrm{I}$ & . & . & . \\
\hline Pulsatilla vernalis & I & I & . & . & . \\
\hline Trifolium montanum & II & . & I & . & . \\
\hline Hieracium hoppeanum & II & . & I & . & . \\
\hline Soldanella pusilla & I & . & $\mathrm{I}$ & . & . \\
\hline Pinus cembra & I & . & I & . & . \\
\hline Hieracium sphaerocephalum & I & . & I & . & . \\
\hline Betula pendula & I & . & I & . & . \\
\hline Hypochaeris uniflora & I & . & I & . & . \\
\hline Dactylorhiza maculata & I & . & I & . & . \\
\hline Phyteuma hemisphaericum & I & . & I & . & . \\
\hline Polygonatum verticillatum & I & . & I & . & . \\
\hline Carex pallescens & I & . & I & . & . \\
\hline Mutellina adonidifolia & II & . & II & III & I \\
\hline Crepis aurea & I & . & II & III & . \\
\hline Leucanthemum ircutianum & I & . & I & I & . \\
\hline Thymus praecox agg. & I & . & $\mathrm{I}$ & I & . \\
\hline Hieracium intybaceum & I & . & . & I & . \\
\hline
\end{tabular}




\begin{tabular}{ll|l|l|l|l}
\hline \multirow{2}{*}{ Assoziation } & \multicolumn{2}{c|}{ Sieversio-Nardetum strictae } & \multicolumn{2}{c}{$\begin{array}{c}\text { Trisetetum } \\
\text { flavescentis }\end{array}$} \\
\hline Subassoziation Nr. & I & 2 & 3 & 4 & 5 \\
\hline Scorzoneroides helvetica & I & $\cdot$ & $\cdot$ & I & $\cdot$ \\
Willemetia stipitata & I & $\cdot$ & II & I & I \\
Carex nigra & I & $\cdot$ & I & I & I \\
Plantago lanceolata & $\cdot$ & I & $\cdot$ & I & $\cdot$ \\
Veronica chamaedrys & $\cdot$ & II & I & II & $\cdot$ \\
Equisetum sylvaticum & $\cdot$ & I & I & I & $\cdot$ \\
Viola canina & $\cdot$ & II & I & $\cdot$ & $\cdot$ \\
Viola biflora & $\cdot$ & $\cdot$ & II & I & $\cdot$ \\
Luzula alpinopilosa & $\cdot$ & $\cdot$ & I & II & $\cdot$ \\
Veronica alpina & $\cdot$ & $\cdot$ & II & I & $\cdot$ \\
Soldanella alpina & $\cdot$ & $\cdot$ & I & I & $\cdot$ \\
Peucedanum ostruthium & $\cdot$ & $\cdot$ & I & II & $\cdot$ \\
Silene dioica & $\cdot$ & $\cdot$ & I & II & III \\
Juncus filiformis & $\cdot$ & $\cdot$ & I & I & I \\
Anthoxanthum odoratum & $\cdot$ & $\cdot$ & $\cdot$ & I & I \\
Dactylus glomerata & $\cdot$ & $\cdot$ & $\cdot$ & I & I \\
Alopecurus pratensis & $\cdot$ & $\cdot$ & $\cdot$ & I & I \\
Trisetum flavescens & $\cdot$ & $\cdot$ & $\cdot$ & I & I \\
Vicia cracca & $\cdot$ & $\cdot$ & I & I \\
Festuca pratensis & $\cdot$ & $\cdot$ & I & I \\
Cirsium heterophyllum & $\cdot$ & $\cdot$ & I & II \\
\hline
\end{tabular}

Seltene Begleiter (Stetigkeit I, Vorkommen nur in einer Subassoziation): Alnus alnobetula (4), Androsace obtusifolia (1), Anthriscus sylvestris (4), Arabis hirsuta (3), Arctostaphylos uva-ursi (1), Bartsia alpina (3), Biscutella laevigata (2), Botrychium lanceolatum (3), Carex canescens (4), Carex echinata (3), Carex frigida (4), Cirsium spinosissimum (4), Coeloglossum viride (1), Danthonia decumbens (1), Empetrum hermaphroditum (1), Equisetum arvense (3), Festuca nigricans (1), Gentiana nivalis (3), Gentiana punctata (1), Hieracium lactucella (1), Huperzia selago (3), Kobresia myosuroides (1), Koeleria hirsuta (1), Larix decidua (2), Luzula campestris (4), Luzula lutea (1), Maianthemum bifolium (1), Melampyrum sylvaticum (1), Molinia caerulea (3), Myosotis palustris (4), Pedicularis recutita (4), Plantago major (2), Phleum alpinum (2), Phleum commutatum (3), Platanthera bifolia (1), Poa angustifolia (4), Poa supina (4), Poa variegata (1), Primula hirsuta (3), Ranunculus nemorosus (4), Rhododendron ferrugineum (3), Rumex acetosella (2), Rumex alpinus (4), Saxifraga paniculata (3), Scorzoneroides autumnale (2), Senecio abrotanifolius (1), Trifolium hybridum (4), Veronica arvense (4), Vicia sepia (4), Viola palustris (4). 


\section{R. Mayer, B. Erschbamer}

\section{Lärchen-Zirbenwälder und Zwergstrauchheiden}

Tab. A1: Stetigkeitstabelle des Obergurgler Zirbenwaldes und der Zwergstrauchheiden im Bereich der Gurgler Heide. Folgende Vegetationstypen wurden unterschieden:

1a: Vaccinio-Pinetum cembrae nardetosum, Ausbildung mit Oxalis acetosella

1b: Vaccinio-Pinetum cembrae nardetosum, Ausbildung mit Loiseleuria procumbens

2: $\quad$ Rhododendretum ferruginei

3: Loiseleurio-Cetrarietum

BS: Baumschicht, SS: Strauchschicht, ZS: Zwergstrauchschicht, KS: Krautschicht, MS: Moos- und Flechtenschicht; C: Charakterarten, D: Differenzialarten.

\begin{tabular}{|c|c|c|c|c|c|c|}
\hline \multicolumn{3}{|c|}{ Vegetationstyp } & 1a & $1 b$ & 2 & 3 \\
\hline \multicolumn{3}{|c|}{ Anzahl der Aufnahmen } & 15 & 5 & 10 & 6 \\
\hline \multicolumn{3}{|c|}{ Mittlere Meereshöhe (m) } & 1981 & 2126 & 2154 & 2193 \\
\hline \multicolumn{3}{|c|}{ Größe der Aufnahmefläche $\left(\mathrm{m}^{2}\right)$} & 200 & 200 & 50 & 25 \\
\hline \multicolumn{3}{|c|}{ Mittlere Kronendeckung (\%) } & 49 & 32 & - & - \\
\hline \multicolumn{3}{|c|}{ Mittlere Artenzahl Gefäßpflanzen } & 19 & 36 & 33 & 19 \\
\hline \multicolumn{3}{|c|}{ Mittlere Artenzahl Kryptogamen } & 8 & 11 & 7 & 8 \\
\hline \multicolumn{3}{|c|}{ Mittlere Artenzahl gesamt } & 27 & 47 & 40 & 27 \\
\hline \multirow[t]{7}{*}{ D 1a } & Oxalis acetosella & KS & III & . & . & . \\
\hline & Festuca arundinacea & KS & III & . & . & . \\
\hline & Gymnocarpium robertianum & KS & II & . & . & . \\
\hline & Letharia vulpina & MS & II & . & . & . \\
\hline & Phyteuma betonicifolium & KS & II & . & . & . \\
\hline & Poa chaixii & KS & II & . & . & . \\
\hline & Dryopteris carthusiana agg. & KS & II & . & . & . \\
\hline \multirow[t]{5}{*}{ D 1a/1b } & Pinus cembra & BS & $\mathrm{V}$ & $\mathrm{V}$ & . & . \\
\hline & Pinus cembra & SS & $\mathrm{V}$ & $\mathrm{V}$ & II & I \\
\hline & Dicranum scoparium & MS & $\mathrm{V}$ & $\mathrm{V}$ & III & I \\
\hline & Calamagrostis villosa & KS & $\mathrm{V}$ & IV & I & . \\
\hline & Polytrichum formosum & MS & III & III & I & I \\
\hline
\end{tabular}




\begin{tabular}{|c|c|c|c|c|c|c|}
\hline \multicolumn{3}{|c|}{ Vegetationstyp } & \multirow{2}{*}{$\frac{\mathbf{1 a}}{\mathrm{IV}}$} & \multirow{2}{*}{$\frac{\mathbf{1 b}}{\mathrm{IV}}$} & \multirow{2}{*}{$\begin{array}{c}2 \\
\mathrm{I}\end{array}$} & \multirow{2}{*}{$\begin{array}{l}3 \\
\mathrm{I}\end{array}$} \\
\hline & Hylocomium splendens & MS & & & & \\
\hline & Racomitrium sudeticum & MS & III & III & . & . \\
\hline & Lonicera caerulea & KS & II & I & . & . \\
\hline & Primula hirsuta & KS & I & II & . & . \\
\hline & Poa nemoralis & KS & I & II & . & . \\
\hline \multirow[t]{7}{*}{ D $\mathbf{1 b}$} & Deschampsia cespitosa & KS & $\mathrm{I}$ & IV & . & . \\
\hline & Festuca rubra agg. & KS & I & IV & I & . \\
\hline & Luzula multiflora & KS & I & III & I & I \\
\hline & Huperzia selago & KS & . & III & . & . \\
\hline & Viola biflora & KS & . & II & . & . \\
\hline & Pinguicula leptoceras & KS & . & II & . & . \\
\hline & Saxifraga aizoides & KS & . & II & . & . \\
\hline \multirow[t]{7}{*}{ D $1 b / 2$} & Leontodon hispidus & KS & I & $\mathrm{V}$ & $\mathrm{V}$ & I \\
\hline & Hieracium glabratum & KS & I & IV & III & I \\
\hline & Nardus stricta & KS & II & IV & IV & $\mathrm{I}$ \\
\hline & Sempervivum montanum & KS & . & III & IV & . \\
\hline & Racomitrium lanuginosum & MS & I & III & IV & . \\
\hline & Coeloglossum viride & KS & . & II & I & . \\
\hline & Alchemilla fissa & KS & . & $\mathrm{I}$ & II & . \\
\hline \multirow[t]{10}{*}{ D 2} & Silene vulgaris & KS & II & II & $\mathrm{V}$ & I \\
\hline & Mutellina adonidifolia & KS & I & II & V & I \\
\hline & Geum montanum & KS & . & II & $\mathrm{V}$ & II \\
\hline & Potentilla aurea & KS & I & II & IV & II \\
\hline & Salix helvetica & SS & . & $\mathrm{I}$ & IV & . \\
\hline & Anthoxanthum odoratum agg. & KS & $\mathrm{I}$ & $\mathrm{I}$ & IV & I \\
\hline & Campanula barbata & KS & I & . & III & . \\
\hline & Lotus corniculatus & KS & I & . & III & . \\
\hline & Ranunculus montanus & KS & I & . & III & . \\
\hline & Barbilophozia lycopodioides & MS & . & . & III & . \\
\hline
\end{tabular}




\begin{tabular}{|c|c|c|c|c|c|c|}
\hline \multicolumn{3}{|c|}{ Vegetationstyp } & \multirow{2}{*}{$\frac{1 \mathrm{a}}{\text {. }}$} & \multirow{2}{*}{$\frac{\mathbf{1 b}}{.}$} & \multirow{2}{*}{$\frac{2}{V}$} & \multirow{2}{*}{$\begin{array}{c}3 \\
\mathrm{~V}\end{array}$} \\
\hline D $2 / 3$ & Luzula lutea & KS & & & & \\
\hline & Euphrasia minima & KS & . & I & III & III \\
\hline & Pulsatilla vernalis & KS & . & . & I & II \\
\hline & Thesium alpinum & KS & . & . & II & I \\
\hline \multirow[t]{7}{*}{ D 3} & Cetraria ericetorum & MS & . & . & . & $\mathrm{V}$ \\
\hline & Carex curvula & KS & . & I & I & $\mathrm{V}$ \\
\hline & Juncus trifidus & KS & . & I & II & V \\
\hline & Cladonia stellaris & MS & . & III & II & V \\
\hline & Hieracium alpinum & KS & $\mathrm{I}$ & . & . & IV \\
\hline & Cetraria nivalis & MS & . & I & . & III \\
\hline & Alectoria ochroleuca & MS & . & . & . & III \\
\hline
\end{tabular}

\section{Vaccinio-Piceetea}

Homogyne alpina

Melampyrum sylvaticum

Rhythidiadelphus triquetrus

\section{Loiseleurio-Vaccinietea}

Vaccinium gaultherioides

Arctostaphylos uva-ursi

\section{Rhododendro-Vaccinion}

Rhododendron ferrugineum

\section{Loiseleurio-Vaccinion}

Empetrum hermaphroditum

Loiseleuria procumbens

\section{Begleiter}

Vaccinium vitis-idaea

Vaccinium myrtillus

Avenella flexuosa

Cladonia rangiferina

Juniperus communis ssp. nana

$\begin{array}{ccccc}\text { KS } & \text { III } & \text { V } & \text { V } & \text { III } \\ \text { KS } & \text { I } & \text { II } & \text { III } & \text {. } \\ \text { MS } & \text { II } & \text { I } & . & \text {. }\end{array}$

$\begin{array}{ccccc}\text { ZS } & \text { V } & \text { V } & \text { V } & \text { V } \\ \text { ZS } & . & \text { I } & \text { I } & \text { I }\end{array}$

$\begin{array}{lllll}\text { ZS } & \mathrm{V} & \mathrm{V} & \mathrm{V} & \text { III }\end{array}$

$\begin{array}{ccccc}\text { ZS } & \text { IV } & \text { V } & \text { V } & \text { V } \\ \text { ZS } & . & \text { IV } & \text { IV } & \text { V }\end{array}$

$\begin{array}{ccccc}\text { ZS } & \text { V } & \text { IV } & \text { V } & \text { V } \\ \text { ZS } & \text { V } & \text { V } & \text { V } & \text { IV } \\ \text { KS } & \text { V } & \text { V } & \text { V } & \text { V } \\ \text { MS } & \text { IV } & \text { III } & \text { IV } & \text { V } \\ \text { ZS } & \text { V } & \text { IV } & \text { V } & \text { III }\end{array}$




\begin{tabular}{|c|c|c|c|c|c|}
\hline Vegetationstyp & & 1a & $\mathbf{1 b}$ & 2 & 3 \\
\hline Pleurozium schreberi & MS & $\mathrm{V}$ & $\mathrm{V}$ & IV & III \\
\hline Cetraria islandica & MS & II & $\mathrm{V}$ & $\mathrm{V}$ & $\mathrm{V}$ \\
\hline Solidago virgaurea ssp. minuta & KS & III & III & IV & I \\
\hline Calluna vulgaris & ZS & III & II & I & I \\
\hline Campanula scheuchzeri & KS & II & III & III & . \\
\hline Atocion rupestre & KS & $\mathrm{I}$ & II & I & $\mathrm{I}$ \\
\hline Agrostis rupestris & KS & I & II & $\mathrm{I}$ & . \\
\hline Phleum rhaeticum & $\mathrm{KS}$ & $\mathrm{I}$ & II & I & . \\
\hline Carex sempervirens & KS & I & I & I & . \\
\hline Racomitrium canescens & MS & I & I & I & . \\
\hline Pinus cembra & KS & I & $\mathrm{V}$ & I & $\mathrm{V}$ \\
\hline Cladonia arbuscula & MS & . & $\mathrm{V}$ & $\mathrm{V}$ & $\mathrm{V}$ \\
\hline Festuca halleri & KS & . & IV & $\mathrm{V}$ & III \\
\hline Avenula versicolor & $\mathrm{KS}$ & . & III & $\mathrm{V}$ & IV \\
\hline Phyteuma hemisphaericum & KS & . & III & III & IV \\
\hline Saxifraga bryoides & KS & . & II & I & III \\
\hline Diphasiastrum alpinum & KS & . & II & I & $\mathrm{I}$ \\
\hline Veronica bellidioides & KS & . & I & II & II \\
\hline Cetraria cucullata & MS & $\mathrm{I}$ & I & . & I \\
\hline Soldanella pusilla & KS & I & I & I & . \\
\hline Gentiana punctata & KS & II & . & III & . \\
\hline Peltigera aphthosa & MS & II & . & II & . \\
\hline Lonicera caerulea & SS & I & . & $\mathrm{I}$ & . \\
\hline Agrostis stolonifera & KS & $\mathrm{I}$ & . & $\mathrm{I}$ & . \\
\hline Alnus alnobetula & SS & $\mathrm{I}$ & . & $\mathrm{I}$ & . \\
\hline $\begin{array}{l}\text { Hypnum cupressiforme var. } \\
\text { cupressiforme }\end{array}$ & MS & I & . & I & . \\
\hline Scorzoneroides helvetica & KS & $\mathrm{I}$ & . & I & . \\
\hline Picea abies & SS & $\mathrm{I}$ & . & $\mathrm{I}$ & . \\
\hline Cirsium spinosissimum & KS & $\mathrm{I}$ & . & II & . \\
\hline
\end{tabular}




\begin{tabular}{|c|c|c|c|c|c|}
\hline \multicolumn{2}{|l|}{ Vegetationstyp } & \multirow{2}{*}{$\frac{\mathbf{1 a}}{\mathrm{II}}$} & \multirow{2}{*}{$\frac{\mathbf{1 b}}{\mathrm{I}}$} & \multirow{2}{*}{$\frac{2}{\mathrm{I}}$} & \multirow{2}{*}{$\frac{3}{\mathrm{I}}$} \\
\hline Luzula luzuloides & KS & & & & \\
\hline Polytrichum piliferum & MS & I & I & I & I \\
\hline Sphagnum squarrosum & MS & I & I & . & . \\
\hline Sorbus aucuparia & BS & I & I & . & . \\
\hline Salix hastata & SS & I & I & . & . \\
\hline Cladonia furcata ssp. furcata & MS & I & I & . & . \\
\hline Persicaria vivipara & KS & . & II & I & . \\
\hline Poa alpina & KS & . & I & I & . \\
\hline Selaginella selaginoides & KS & . & I & I & . \\
\hline Trifolium pratense ssp. nivale & KS & . & I & I & . \\
\hline Oreochloa disticha & KS & . & I & . & I \\
\hline Festuca varia & KS & . & I & . & I \\
\hline Leucanthemopsis alpina & KS & . & I & . & II \\
\hline Stereocaulon alpinum & MS & . & I & . & II \\
\hline Pyrola minor & KS & . & . & II & . \\
\hline Salix herbacea & KS & . & . & II & . \\
\hline Senecio incanus ssp. carniolicus & KS & . & . & II & I \\
\hline Thamnolia vermicularis & MS & . & . & I & I \\
\hline Achillea moschata & KS & . & . & I & I \\
\hline Antennaria dioica & KS & . & . & I & I \\
\hline
\end{tabular}

\section{Seltene Begleiter (Stetigkeit I, Vorkommen nur in einem Syntaxon):}

Agrostis capillaris (1b), Ajuga pyramidalis (1a), Alnus alnobetula (1a), Androsace obtusifolia (2), Arnica montana (1a), Asplenium septentrionalis (1a), Bartsia alpina (1b), Betula pendula (SS; 1a), Calliergonella cuspidata (MS; 1a), Carex capillaris (1b), Carex davalliana (1b), Carex echinata (1b), Carex frigida (1b), Carex nigra (1b), Cladonia uncinalis (MS; 3), Cystopteris montana (1a), Dicranoweisia crispula (MS; 2), Dicranella heteromella (MS; 2), Dicranum elongata (MS; 3), Dicranum fuscescens (MS; 1b), Doronicum clusii (1a), Dryopteris expansa (1b), Epilobium angustifolium (1b), Festuca norica (1b), Galium anisophyllon (1a), Galium sylvaticum (1a), Gentiana acaulis (1a), Gentianella anisodonta (2), Geranium pratense (1a), Geranium sylvaticum (1a), Gymnocarpium dryopteris (1b), Hieracium bifidum (1a), Hieracium murorum (1a), Hieracium sp. (1a), Hieracium sphaerocephalum (2), Larix decidua (BS; 1b), Larix decidua (SS; 1a), Leontodon hispidus ssp. hyoseroides (2), Lophozia ventricosa (MS; 2), Luzula alpinopilosa (2), Luzula sylvatica ssp. sieberi (1b), Lycopodium annotinum (1b), Orthodicranum montanum (MS; 1a), 
Anhang Kapitel 4 I Lärchen-Zirbenwälder und Zwergstrauchheiden

Orthotrichum sp. (MS; 2), Parnassia palustris (1b), Pedicularis tuberosa (2), Peucedanum ostruthium (1a), Picea abies (BS; 1a), Pinus mugo (SS; 1a), Polypodium vulgare (1a), Polystichum lonchitis (1b), Polytrichum juniperinum (MS; 2), Potentilla erecta (1a), Primula glutinosa (1b), Pseudorchis albida (1b), Rumex alpinus (1a), Salix laggeri (SS; 1b), Saxifraga sp. (3), Sibbaldia procumbens (2), Silene acaulis (3), Sphagnum nemorum (MS; 1b), Thymus praecox agg. (1a), Trichophorum cespitosum (1b), Trifolium alpinum (2), Trifolium repens (2), Trollius europaeus (1b), Willemetia stipitata (1b). 
